\title{
Supporting Information Regio- and Stereoselective Alkylboration of Endocyclic Olefins Enabled by Nickel Catalysis
}

\author{
Chao Ding, Yaoyu Ren, Caocao Sun, Jiao Long, Guoyin Yin*
}

The Institute for Advanced Studies, Wuhan University, Wuhan, Hubei 430072, P. R. China

*Email: yinguoyin@whu.edu.cn 


\section{Table of Contents}

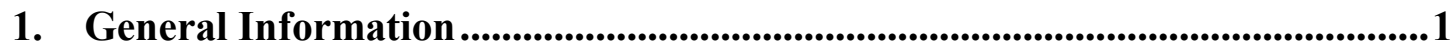

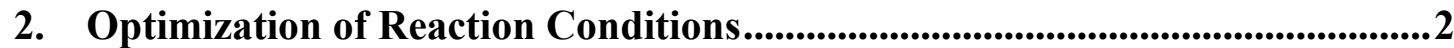

3. General Procedure for the Migratory Alkylboration of Cyclic Olefins ............4

4. Analytical Data of Compounds........................................................................................5

5. Scale-up Experiment and Transformations of Products ...................................43

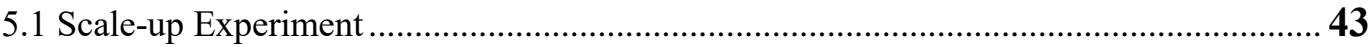

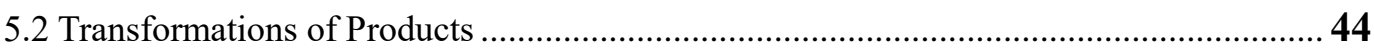

6. Synthetic Applications ..........................................................................................................551

7. Mechanism Studies ...............................................................................................................54

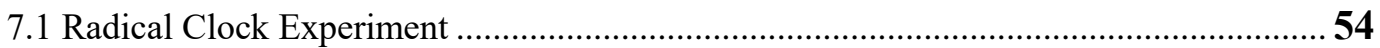

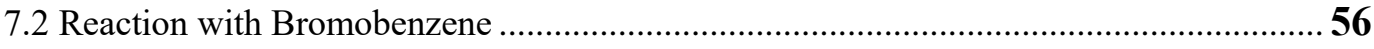

8. X-ray Structures and Data .......................................................................5

9. NMR Spectra .......................................................................................................................61

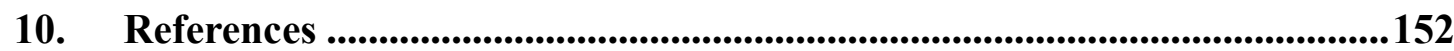




\section{General Information}

General information: All reactions were run under a dry argon atmosphere fitted on a glass tube or vial. All glassware was oven dried at $120{ }^{\circ} \mathrm{C}$ for $2 \mathrm{~h}$ and cooled down under vacuum. Thin layer chromatography (TLC) employed glass $0.25 \mathrm{~mm}$ silica gel plates. Flash chromatography columns were packed with 200-300 mesh silica gel in petroleum (bp. 60-90 $\left.{ }^{\circ} \mathrm{C}\right)$. GC-MS spectra were recorded on a Varian GC-MS 3900-2100 T. GC analyses were performed on an Agilent 7890B gas chromatograph with an FID detector using a J \& W DB-1 column (10 m, $0.1 \mathrm{~mm}$ I.D.). The high-resolution mass spectra were measured on Thermo Fisher Scientific Exactive Plus (ESI). Optical rotation was determined using a Perkin Elmer 343 polarimeter. HPLC analysis was conducted on an Agilent 1260 Series instrument. All new compounds were characterized by ${ }^{1} \mathrm{H}$ NMR, ${ }^{13} \mathrm{C}$ NMR, ${ }^{19} \mathrm{~F}$ NMR and HRMS. The known compounds were characterized by ${ }^{1} \mathrm{H}$ NMR, ${ }^{13} \mathrm{C}$ NMR. ${ }^{1} \mathrm{H},{ }^{13} \mathrm{C}$ and ${ }^{19} \mathrm{~F}$ NMR data were recorded with JNM-ECZ 400 and Bruker $600 \mathrm{MHz}$ with tetramethylsilane as an internal standard. Data for ${ }^{1} \mathrm{H},{ }^{13} \mathrm{C}$ and ${ }^{19} \mathrm{~F}$ NMR are reported as follows: chemical shift ( $\delta$ ppm), multiplicity ( $\mathrm{s}=$ singlet, $\mathrm{d}=$ doublet, $\mathrm{t}=$ triplet, $\mathrm{q}=$ quartet, $\mathrm{dd}=$ doublet of doublet, $\mathrm{dt}=$ doublet of triplet, $\mathrm{dq}$ $=$ doublet of quartet, $\mathrm{m}=$ multiplet $)$, integration, and coupling constant $(\mathrm{Hz})$. All chemical shifts $(\delta)$ were reported in ppm and coupling constants $(J)$ in Hz. All chemical shifts were reported relative to tetramethylsilane $\left(0 \mathrm{ppm}\right.$ for $\left.{ }^{1} \mathrm{H}\right)$, Chloroform- $d\left(77.16 \mathrm{ppm}\right.$ for $\left.{ }^{13} \mathrm{C}\right)$, respectively.

Materials: $\mathrm{NiCl}_{2} \cdot \mathrm{DME}$ (CAS: 29046-78-4), LiOMe were purchased from Energy Chemical. Anhydrous dioxane were purchased from Adamas-beta ${ }^{\circledR}$. Some alkenes were synthesized according to the references. Unless otherwise noted, alkene, alkyl bromide and bis(pinacolato)diboron were obtained from commercial suppliers (Energy Chemical, Adamasbeta ${ }^{\circledR}, J \& K$ and Innochem so on) and used without further purification. 


\section{Optimization of Reaction Conditions}

Table S1. Effect of Ligands ${ }^{a b}$

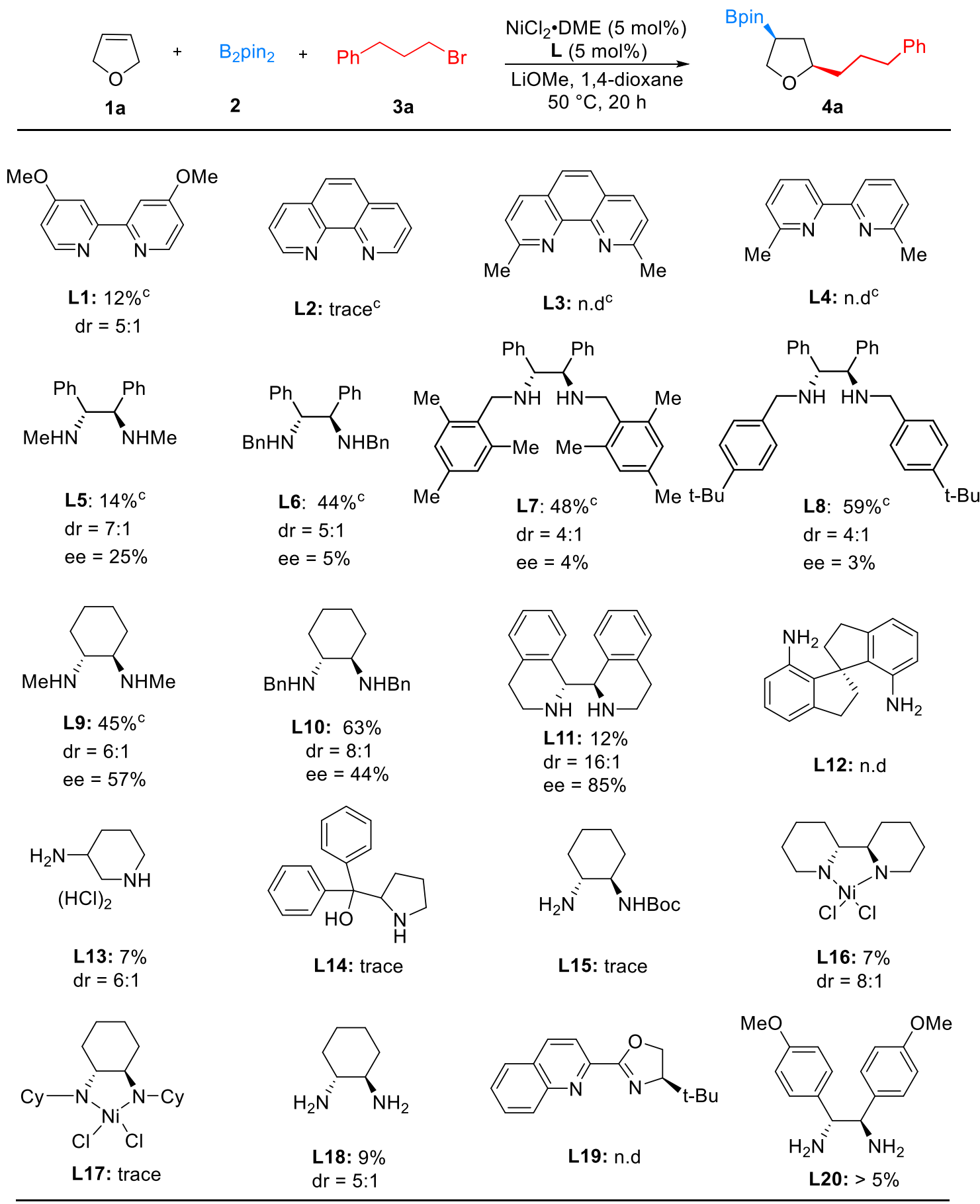

[a] Conditions:1a ( $0.4 \mathrm{mmol}, 1.0$ equiv), 2 ( $0.6 \mathrm{mmol}, 1.5$ equiv), $3 \mathrm{a}$ ( $0.6 \mathrm{mmol}, 1.5$ equiv),

$\mathrm{NiCl}_{2} \cdot \operatorname{DME}(5 \mathrm{~mol} \%), \mathbf{L}$ ( $\left.5 \mathrm{~mol} \%\right), \operatorname{LiOMe}(0.6 \mathrm{mmol}, 1.5$ equiv), 1,4-dioxane ( $2 \mathrm{~mL}), 50^{\circ} \mathrm{C}$, 20 h. [b] GC yields against naphthalene. Enantiomeric excess (ee) of the corresponding alcohol after oxidation. [c] NMP instead of 1,4-dioxane. 
Table S2. Effect of solvents ${ }^{a b}$

\begin{tabular}{|c|c|c|c|c|c|}
\hline$\overline{0}_{0}$ & $\begin{array}{c}\mathrm{B}_{2} \mathrm{pin}_{2} \\
\mathbf{2}\end{array}$ & $\widehat{\mathrm{Br}}$ & $\left.\begin{array}{c}\mathrm{NiCl}_{2} \cdot \operatorname{DME}(5 \mathrm{~mol} \%) \\
\mathrm{L}(5 \mathrm{~mol} \%)\end{array}\right)$ & Bpin & 'p \\
\hline Entry & Ligand & Solvent & Yield (\%) & $\mathrm{dr}$ & ee $(\%)$ \\
\hline 1 & L10 & NMP & 90 & $19: 1$ & 36 \\
\hline 2 & L10 & THF & 74 & $11: 1$ & 36 \\
\hline 3 & L10 & MTBE & 31 & $8: 1$ & 40 \\
\hline 4 & L10 & 1,4-dioxane & 63 & $8: 1$ & 44 \\
\hline 5 & L11 & NMP & 39 & $8: 1$ & 71 \\
\hline 6 & L11 & 2-Me-THF & 15 & $12: 1$ & 58 \\
\hline 7 & L11 & 1,4-dioxane & 12 & $16: 1$ & 85 \\
\hline $8^{c}$ & L11 & 1,4-dioxane & 14 & $16: 1$ & 91 \\
\hline $9^{d}$ & L11 & 1,4-dioxane & 31 & $20: 1$ & 94 \\
\hline $10^{c d}$ & L11 & 1,4-dioxane & 75 & 25:1 & 94 \\
\hline
\end{tabular}

[a] Conditions:1a ( $0.4 \mathrm{mmol}, 1.0$ equiv), 2 ( $0.6 \mathrm{mmol}, 1.5$ equiv), $3 \mathrm{a}$ ( $0.6 \mathrm{mmol}, 1.5$ equiv), $\mathrm{NiCl}_{2} \cdot \operatorname{DME}(5 \mathrm{~mol} \%), \mathbf{L}(5 \mathrm{~mol} \%)$, LiOMe ( $0.6 \mathrm{mmol}, 1.5$ equiv), 1,4 -dioxane $(2 \mathrm{~mL}), 50{ }^{\circ} \mathrm{C}$, 20 h. [b] GC yields against naphthalene. Enantiomeric excess (ee) of the corresponding alcohol after oxidation. [c] 2 (1.4 mmol, 3.5 equiv), LiOMe (1.4 mmol, 3.5 equiv). [d] 0.6 equiv KI was added. 


\section{General Procedure for the Migratory Alkylboration of Cyclic Olefins}

\section{General Procedure A:}
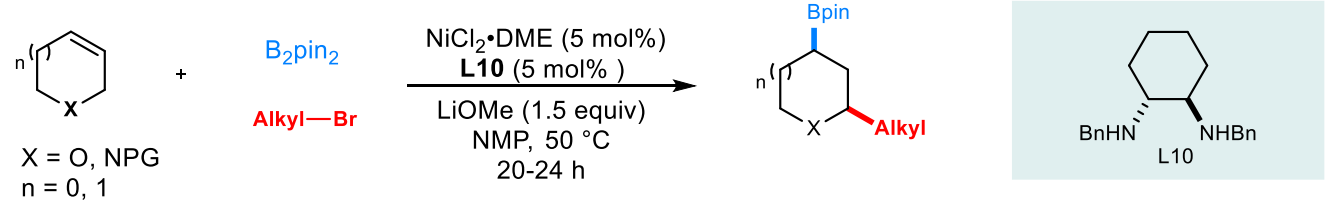

Under $\mathrm{N}_{2}$ atmosphere, an oven-dried $10 \mathrm{~mL}$ reaction tube which equipped with a magnetic stir bar and sealed with a rubber stopper sequentially was added $\mathrm{NiCl}_{2} \cdot \mathrm{DME}(4.4 \mathrm{mg}, 0.02 \mathrm{mmol}$, $5 \mathrm{~mol} \%$ ), L10 (6.0 mg, $0.02 \mathrm{mmol}, 5 \mathrm{~mol} \%)$, LiOMe (22.8 mg, $0.6 \mathrm{mmol}, 1.5$ equiv) and bis(pinacolato)diboron (152.4 mg, $0.6 \mathrm{mmol}, 1.5$ equiv). Then anhydrous NMP (1 mL), alkenes ( 0.4 mmol, 1.0 equiv), alkylbromide ( $0.6 \mathrm{mmol}, 1.5$ equiv), anhydrous NMP $(1 \mathrm{~mL})$ were added and the mixture was stirred. After $20-24 \mathrm{~h}$ of stirring at $50{ }^{\circ} \mathrm{C}$, the resulting mixture was quenched with water $(3 \mathrm{~mL})$ and further diluted with ethyl acetate $(3 \mathrm{~mL})$. Then the mixture was extracted with ethyl acetate and the combined organic layers were dried over anhydrous $\mathrm{Na}_{2} \mathrm{SO}_{4}$, filtered, and concentrated under vacuum. The crude material was separated on a silica gel column affording the desired product. The product was oxidized according to reported literature. ${ }^{1,2}$

\section{General Procedure B:}
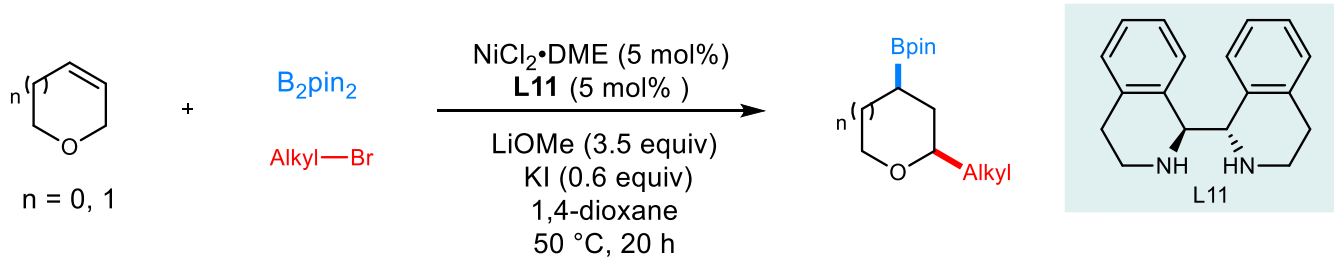

Under $\mathrm{N}_{2}$ atmosphere, an oven-dried $10 \mathrm{~mL}$ reaction tube which equipped with a magnetic stir bar and sealed with a rubber stopper sequentially was added $\mathrm{NiCl}_{2} \cdot \mathrm{DME}(4.4 \mathrm{mg}, 0.02 \mathrm{mmol}$, $5 \mathrm{~mol} \%$ ), L11 (5.3 mg, $0.02 \mathrm{mmol}, 5 \mathrm{~mol} \%$ ), LiOMe (53.2 mg, $1.4 \mathrm{mmol}, 3.5$ equiv), KI (40.0 $\mathrm{mg}, 0.24 \mathrm{mmol}, 0.6$ equiv) and bis(pinacolato)diboron ( $355.6 \mathrm{mg}, 1.4 \mathrm{mmol}, 3.5$ equiv). Then anhydrous 1,4-dioxane $(1 \mathrm{~mL})$, alkenes $(0.4 \mathrm{mmol}, 1.0$ equiv), alkylbromide ( $0.6 \mathrm{mmol}, 1.5$ equiv), anhydrous 1,4-dioxane ( $1 \mathrm{~mL})$ were added and the mixture was stirred. After $20 \mathrm{~h}$ of stirring at $50{ }^{\circ} \mathrm{C}$, the resulting mixture was quenched with water $(3 \mathrm{~mL})$ and further diluted with 
ethyl acetate $(3 \mathrm{~mL})$. Then the mixture was extracted with ethyl acetate and the combined organic layers were dried over anhydrous $\mathrm{Na}_{2} \mathrm{SO}_{4}$, filtered, and concentrated under vacuum. The crude material was separated on a silica gel column affording the desired product. The Enantiomeric excess (ee) value was determined by HPLC with chiral column after oxidized to the corresponding alcohol. ${ }^{1,2}$

\section{Analytical Data of Compounds}

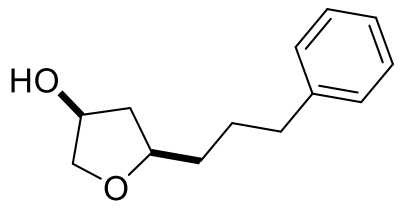

cis-5-(3-phenylpropyl)-tetrahydrofuran-3-ol (4a). This compound was synthesized according to general procedure A. The residue was purified by column chromatography on silica gel to afford the product 4a $(68.5 \mathrm{mg}, 83 \%$ yield, $\mathrm{dr}=19: 1)$ as a colorless oil; ${ }^{1} \mathbf{H}$ NMR $(600 \mathrm{MHz}$, Chloroform-d) $\delta 7.32$ - $7.24(\mathrm{~m}, 2 \mathrm{H}), 7.20$ - $7.15(\mathrm{~m}, 3 \mathrm{H}), 4.46$ - $4.41(\mathrm{~m}, 1 \mathrm{H}), 3.89$ - $3.74(\mathrm{~m}$, 2H), $3.66(\mathrm{dd}, J=9.9,4.4 \mathrm{~Hz}, 1 \mathrm{H}), 2.65$ (ddd, $J=8.2,6.6,2.8 \mathrm{~Hz}, 2 \mathrm{H}), 2.32$ (ddd, $J=13.4$, 7.6, $6.8 \mathrm{~Hz}, 1 \mathrm{H}), 1.84-1.71(\mathrm{~m}, 3 \mathrm{H}), 1.70-1.66(\mathrm{~m}, 1 \mathrm{H}), 1.64-1.58(\mathrm{~m}, 1 \mathrm{H}), 1.49$ (dddd, $J=$ 13.5, 7.3, 3.0, $1.4 \mathrm{~Hz}, 1 \mathrm{H}) \mathrm{ppm} ;{ }^{13}$ C NMR (151 MHz, Chloroform-d) $\delta$ 142.4, 128.5, 128.4, 125.9, 79.2, 75.5, 72.6, 41.5, 35.9, 35.8, 28.2 ppm; HRMS (ESI) calculated $[\mathrm{M}+\mathrm{H}]^{+}$for $\mathrm{C}_{13} \mathrm{H}_{19} \mathrm{O}_{2}^{+}=207.1380$, found: 207.1376.

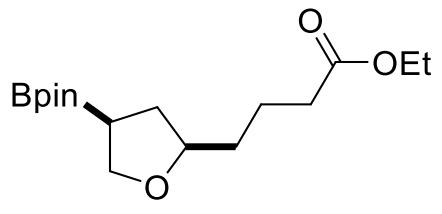

cis-ethyl4-(4-(4,4,5,5-tetramethyl-1,3,2-dioxaborolan-2-yl)tetrahydrofuran-2-yl)-butanoate (4b). This compound was synthesized according to general procedure A. The residue was purified by column chromatography on silica gel to afford the product $4 \mathbf{b}(105.0 \mathrm{mg}, 85 \%$ yield, $\mathrm{dr}=16: 1)$ as a colorless oil; ${ }^{1} \mathbf{H}$ NMR (600 MHz, Chloroform- $\left.d\right) \delta 4.12(\mathrm{q}, J=7.1 \mathrm{~Hz}, 2 \mathrm{H})$, $3.95(\mathrm{dd}, J=8.8,8.1 \mathrm{~Hz}, 1 \mathrm{H}), 3.80(\mathrm{ddt}, J=9.2,6.8,5.5 \mathrm{~Hz}, 1 \mathrm{H}), 3.76(\mathrm{dd}, J=9.8,8.1 \mathrm{~Hz}$, 1H), 2.33 (ddd, $J=8.0,6.9,3.7 \mathrm{~Hz}, 2 \mathrm{H}), 2.13(\mathrm{ddd}, J=12.0,7.7,5.5 \mathrm{~Hz}, 1 \mathrm{H}), 1.80-1.61(\mathrm{~m}$, 4H), 1.51 (ddt, $J=13.2,11.0,5.3 \mathrm{~Hz}, 1 \mathrm{H}), 1.42(\mathrm{td}, J=11.8,9.2 \mathrm{~Hz}, 1 \mathrm{H}), 1.26-1.23(\mathrm{~m}, 15 \mathrm{H})$ ppm; ${ }^{13}$ C NMR (151 MHz, Chloroform-d) $\delta$ 173.8, 83.5, 80.1, 69.8, 60.4, 35.1, 35.0, 34.5, 24.9, 22.1, 14.4 ppm; ${ }^{11}$ B NMR (128 MHz, Chloroform-d) $\delta 33.51$ ppm; HRMS (ESI) calculated $[\mathrm{M}+\mathrm{H}]^{+}$for $\mathrm{C}_{16} \mathrm{H}_{30} \mathrm{BO}_{5}{ }^{+}=313.2181$, found: 313.2182 . 


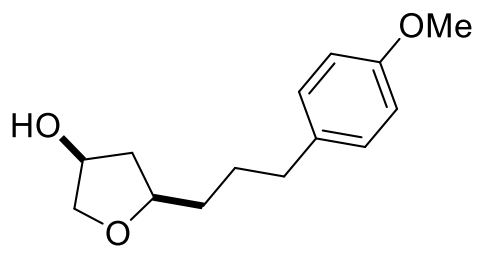

cis-5-(3-(4-methoxyphenyl)-propyl)-tetrahydrofuran-3-ol

(4c). This compound was synthesized according to general procedure A. The residue was purified by column chromatography on silica gel to afford the product $\mathbf{4 c}(70.0$ mg, 74\% yield, $\mathrm{dr}>20: 1)$ as a colorless oil; ${ }^{1} \mathbf{H}$ NMR (600 MHz, Chloroform- $\left.\boldsymbol{d}\right) \delta 7.11-7.07$ $(\mathrm{m}, 2 \mathrm{H}), 6.83-6.80(\mathrm{~m}, 2 \mathrm{H}), 4.44-4.40(\mathrm{~m}, 1 \mathrm{H}), 3.83-3.79(\mathrm{~m}, 2 \mathrm{H}), 3.78(\mathrm{~s}, 3 \mathrm{H}), 3.66(\mathrm{dd}$, $J=9.9,4.4 \mathrm{~Hz}, 1 \mathrm{H}), 2.59$ (ddd, $J=7.8,6.5,2.6 \mathrm{~Hz}, 2 \mathrm{H}), 2.32(\mathrm{ddd}, J=13.4,7.6,6.8 \mathrm{~Hz}, 1 \mathrm{H})$, $1.88(\mathrm{~s}, 1 \mathrm{H}), 1.77-1.70(\mathrm{~m}, 2 \mathrm{H}), 1.66-1.57(\mathrm{~m}, 2 \mathrm{H}), 1.48$ (dddd, $J=13.5,7.4,3.0,1.4 \mathrm{~Hz}$, 1H) ppm; ${ }^{13}$ C NMR (151 MHz, Chloroform-d) $\delta$ 157.8, 134.5, 129.4, 113.8, 79.2, 75.5, 72.6, 55.4, 41.6, 35.8, 35.0, 28.4 ppm; HRMS (ESI) calculated $[\mathrm{M}+\mathrm{H}]^{+}$for $\mathrm{C}_{14} \mathrm{H}_{21} \mathrm{O}_{3}{ }^{+}=237.1485$, found: 237.1482 .

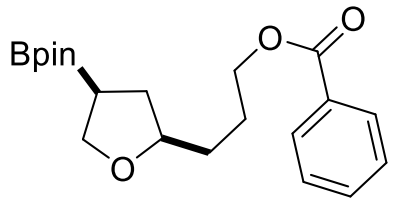

cis-3-(4-(4,4,5,5-tetramethyl-1,3,2-dioxaborolan-2-yl)-tetrahydrofuran-2-yl)-propyl benzoate (4d). This compound was synthesized according to general procedure A. The residue was purified by column chromatography on silica gel to afford the product $\mathbf{4 d}(89.0 \mathrm{mg}, 62 \%$ yield, $\mathrm{dr}=18: 1)$ as a colorless oil; ${ }^{1} \mathbf{H}$ NMR (400 MHz, Chloroform- $\left.\boldsymbol{d}\right) \delta 8.06-7.98(\mathrm{~m}, 2 \mathrm{H}), 7.58$ 7.48 (m, 1H), $7.46-7.37$ (m, 2H), 4.32 (td, $J=6.5,1.7 \mathrm{~Hz}, 2 \mathrm{H}), 3.95$ (t, $J=8.4 \mathrm{~Hz}, 1 \mathrm{H}), 3.85$ (ddt, $J=9.1,6.9,5.5 \mathrm{~Hz}, 1 \mathrm{H}), 3.76(\mathrm{dd}, J=9.7,8.1 \mathrm{~Hz}, 1 \mathrm{H}), 2.14(\mathrm{ddd}, J=12.0,7.8,5.5 \mathrm{~Hz}$, 1H), $1.97-1.71(\mathrm{~m}, 3 \mathrm{H}), 1.73-1.55(\mathrm{~m}, 2 \mathrm{H}), 1.44(\mathrm{td}, J=11.8,9.2 \mathrm{~Hz}, 1 \mathrm{H}), 1.22(\mathrm{~s}, 12 \mathrm{H})$ ppm; ${ }^{13}$ C NMR (101 MHz, Chloroform-d) $\delta$ 166.7, 132.9, 130.5, 129.7, 128.4, 83.5, 79.9, 69.8, 65.1, 35.1, 32.1, 25.9, 24.8 ppm; ${ }^{11}$ B NMR (128 MHz, Chloroform-d) $\delta 32.51$ ppm; HRMS (ESI) calculated $[\mathrm{M}+\mathrm{H}]^{+}$for $\mathrm{C}_{20} \mathrm{H}_{30} \mathrm{BO}_{5}{ }^{+}=361.2181$, found: 361.2182 .

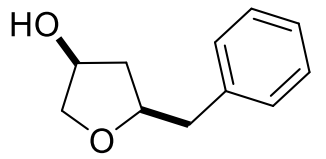

cis-5-benzyltetrahydrofuran-3-ol (4e). This compound was synthesized according to general procedure A. The residue was purified by column chromatography on silica gel to afford the product $4 \mathbf{e}(64.4 \mathrm{mg}, 90 \%$ yield, $\mathrm{dr}>$ 20:1) as a colorless oil; ${ }^{\mathbf{1}} \mathbf{H}$ NMR (400 MHz, Chloroform- $\left.\boldsymbol{d}\right) \delta$ $7.34-7.28$ (m, 2H), 7.25 - 7.20 
(m, 3H), 4.39 (tq, $J=4.4,2.1 \mathrm{~Hz}, 1 \mathrm{H}), 4.12(\mathrm{dq}, J=8.1,6.4 \mathrm{~Hz}, 1 \mathrm{H}), 3.85(\mathrm{dt}, J=9.9,1.7 \mathrm{~Hz}$, 1H), $3.68(\mathrm{dd}, J=9.8,4.3 \mathrm{~Hz}, 1 \mathrm{H}), 3.01(\mathrm{dd}, J=13.7,6.3 \mathrm{~Hz}, 1 \mathrm{H}), 2.92(\mathrm{dd}, J=13.7,6.2 \mathrm{~Hz}$, 1H), 2.26 (ddd, $J=14.0,7.8,6.6 \mathrm{~Hz}, 1 \mathrm{H}), 1.77$ (s, 1H), 1.63 (dddd, J = 13.7, 6.7, 2.8, $1.4 \mathrm{~Hz}$, 1H) ppm; ${ }^{13} \mathbf{C}$ NMR (101 MHz, $\left.\mathbf{C D C l}_{3}\right) \delta$ 138.4, 129.5, 128.6, 126.6, 79.6, 75.7, 72.5, 42.1, 40.4 ppm; HRMS (ESI) calculated $[\mathrm{M}+\mathrm{H}]^{+}$for $\mathrm{C}_{11} \mathrm{H}_{15} \mathrm{O}_{2}{ }^{+}=179.1067$, found: 179.1066 .

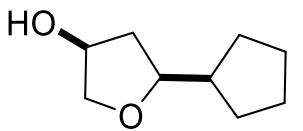
cis-5-cyclopentyltetrahydrofuran-3-ol (4f). This compound was synthesized according to general procedure A. The residue was purified by column chromatography on silica gel to afford the product $\mathbf{4 f}(29.0 \mathrm{mg}, 48 \%$ yield, $\mathrm{dr}=8: 1)$ as a colorless oil; ${ }^{1} \mathbf{H}$ NMR (600 MHz, Chloroform- $\left.d\right) \delta 4.47-4.42(\mathrm{~m}, 1 \mathrm{H}), 3.84(\mathrm{dt}, J=9.8$, $1.6 \mathrm{~Hz}, 1 \mathrm{H}), 3.67(\mathrm{dd}, J=9.9,4.4 \mathrm{~Hz}, 1 \mathrm{H}), 3.59(\mathrm{q}, J=7.8 \mathrm{~Hz}, 1 \mathrm{H}), 2.32(\mathrm{ddd}, J=13.5,7.5$, $6.8 \mathrm{~Hz}, 1 \mathrm{H}), 2.10$ - $2.01(\mathrm{~m}, 1 \mathrm{H}), 1.90$ - $1.84(\mathrm{~m}, 1 \mathrm{H}), 1.75$ - 1.67 (m, 2H), 1.65 - 1.59 (m, 2H), 1.58 - $1.53(\mathrm{~m}, 3 \mathrm{H}), 1.43$ - 1.34 (m, 1H), 1.23 - $1.16(\mathrm{~m}, 1 \mathrm{H}) \mathrm{ppm} ;{ }^{13} \mathbf{C} \mathbf{~ N M R}\left(\mathbf{1 5 1} \mathbf{~ M H z}, \mathbf{C D C l}_{3}\right)$ $\delta$ 83.7, 75.6, 72.8, 45.5, 40.7, 30.2, 29.2, 25.7, 25.6 ppm; HRMS (ESI) calculated $[\mathrm{M}+\mathrm{H}]^{+}$for $\mathrm{C}_{9} \mathrm{H}_{17} \mathrm{O}_{2}{ }^{+}=157.1223$, found: 157.1223 .

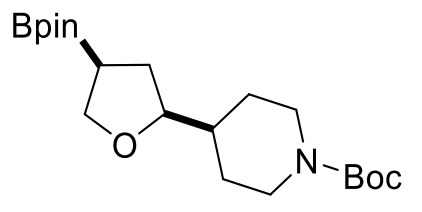

cis-tert-butyl-4-(4-(4,4,5,5-tetramethyl-1,3,2-dioxaborolan-2yl)-tetrahydrofuran-2-yl)-piperidine-1-carboxylate (4g). This compound was synthesized according to general procedure A. The residue was purified by column chromatography on silica gel to afford the product $\mathbf{4 g}$ (78.6 mg, 52\% yield, dr > 20:1) as a white solid; ${ }^{1}$ H NMR (400 MHz, Chloroform-d) $\delta 4.09$ (s, 2H), $3.97-3.92(\mathrm{~m}, 1 \mathrm{H}), 3.74-3.60(\mathrm{~m}, 1 \mathrm{H}), 3.54-3.41(\mathrm{~m}, 1 \mathrm{H}), 2.69-2.57$ (m, 2H), 2.04 (ddd, $J$ $=12.5,7.5,5.5 \mathrm{~Hz}, 1 \mathrm{H}), 1.85(\mathrm{dt}, J=12.9,2.9 \mathrm{~Hz}, 1 \mathrm{H}), 1.72-1.61(\mathrm{~m}, 1 \mathrm{H}), 1.58-1.44(\mathrm{~m}$, 3H), $1.41(\mathrm{~d}, J=1.1 \mathrm{~Hz}, 9 \mathrm{H}), 1.21(\mathrm{~d}, J=1.1 \mathrm{~Hz}, 12 \mathrm{H}), 1.17-1.09(\mathrm{~m}, 2 \mathrm{H}) \mathrm{ppm} ;{ }^{13} \mathbf{C} \mathbf{N M R}$ $\left(\mathbf{1 0 1} \mathbf{M H z}, \mathbf{C D C l}_{\mathbf{3}}\right) \delta 155.0,84.2,83.5,79.3,69.9,43.8,41.6,32.9,28.6,28.2,24.8$ ppm; ${ }^{11} \mathbf{B}$ NMR (128 MHz, Chloroform-d) $\delta 34.28$ ppm; HRMS (ESI) calculated $[\mathrm{M}+\mathrm{H}]^{+}$for $\mathrm{C}_{20} \mathrm{H}_{37} \mathrm{BNO}_{5}^{+}=382.2759$, found: 382.2757 .

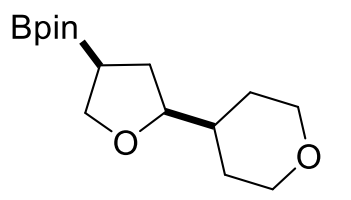

cis-4,4,5,5-tetramethyl-2-(5-(tetrahydro-2H-pyran-4-yl)-tetrahy- 
drofuran-3-yl)-1,3,2-dioxaborolane (4h). This compound was synthesized according to general procedure A. The residue was purified by column chromatography on silica gel to afford the product $\mathbf{4 h}(61.8 \mathrm{mg}, 55 \%$ yield, $\mathrm{dr}=10: 1)$ as a colorless oil; ${ }^{\mathbf{1}} \mathbf{H} \mathbf{N M R}(\mathbf{4 0 0} \mathbf{~ M H z}$, Chloroform-d) $\delta 3.99-3.90(\mathrm{~m}, 3 \mathrm{H}), 3.69(\mathrm{dd}, J=10.0,8.0 \mathrm{~Hz}, 1 \mathrm{H}), 3.57-3.46(\mathrm{~m}, 1 \mathrm{H})$, 3.34 (tdd, $J=11.8,4.6,2.4 \mathrm{~Hz}, 2 \mathrm{H}), 2.05$ (ddd, $J=12.0,7.5,5.4 \mathrm{~Hz}, 1 \mathrm{H}), 1.83-1.76(\mathrm{~m}, 1 \mathrm{H})$, $1.73-1.64(\mathrm{~m}, 1 \mathrm{H}), 1.62-1.52(\mathrm{~m}, 1 \mathrm{H}), 1.50-1.41(\mathrm{~m}, 2 \mathrm{H}), 1.40-1.30(\mathrm{~m}, 2 \mathrm{H}), 1.21(\mathrm{~s}, 13 \mathrm{H})$ ppm; ${ }^{13} \mathbf{C}$ NMR (101 MHz, $\left.\mathbf{C D C l}_{3}\right) \delta 84.4,83.5,69.9,68.0,67.8,40.6,32.7,30.5,29.0,24.9$ ppm; ${ }^{11}$ B NMR (128 MHz, Chloroform- $\boldsymbol{d}$ ) $\delta 32.67$ ppm; HRMS (ESI) calculated $[\mathrm{M}+\mathrm{H}]^{+}$for $\mathrm{C}_{15} \mathrm{H}_{28} \mathrm{BO}_{4}{ }^{+}=283.2075$, f o u n : 283.2076.

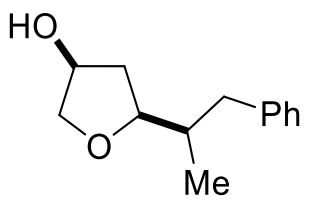

cis-5-(1-phenylpropan-2-yl)-tetrahydrofuran-3-ol (4i). This compound was synthesized according to general procedure A. The residue was purified by column chromatography on silica gel to afford the product $4 \mathbf{i}(50.5 \mathrm{mg}, 61 \%$ yield, $\mathrm{dr}=1.2: 1)$ as a colorless oil; ${ }^{1} \mathbf{H}$ NMR $(400 \mathrm{MHz}$, Chloroform- $d) \delta 7.32-7.25(\mathrm{~m}, 4 \mathrm{H}), 7.22-7.14(\mathrm{~m}, 7 \mathrm{H}), 4.46(\mathrm{dddd}, J=10.0,5.2,3.2,2.0$ $\mathrm{Hz}, 2 \mathrm{H}), 3.85(\mathrm{tt}, J=9.8,1.7 \mathrm{~Hz}, 2 \mathrm{H}), 3.72-3.63(\mathrm{~m}, 3 \mathrm{H}), 3.57$ (q, $J=7.7 \mathrm{~Hz}, 1 \mathrm{H}), 3.00(\mathrm{dd}$, $J=13.3,4.5 \mathrm{~Hz}, 1 \mathrm{H}), 2.83(\mathrm{dd}, J=13.3,5.0 \mathrm{~Hz}, 1 \mathrm{H}), 2.44-2.24(\mathrm{~m}, 4 \mathrm{H}), 2.08-1.92(\mathrm{~m}, 2 \mathrm{H})$, $1.86(\mathrm{~s}, 2 \mathrm{H}), 1.68-1.56(\mathrm{~m}, 2 \mathrm{H}), 0.93(\mathrm{~d}, J=6.7 \mathrm{~Hz}, 3 \mathrm{H}), 0.81(\mathrm{~d}, J=6.7 \mathrm{~Hz}, 3 \mathrm{H}) \mathrm{ppm} ;{ }^{13} \mathbf{C}$ NMR (101 MHz, $\left.\mathbf{C D C l}_{3}\right) \delta 140.8,140.6,129.5,129.3,128.4,128.3,126.0,125.9,83.0,82.8$, 75.46, 75.45, 72.48, 72.46, 39.9, 39.8, 39.7, 39.3, 39.1, 15.3, 15.1 ppm; HRMS (ESI) calculated $[\mathrm{M}+\mathrm{H}]^{+}$for $\mathrm{C}_{13} \mathrm{H}_{19} \mathrm{O}_{2}{ }^{+}=207.1380$, found: 207.1380.

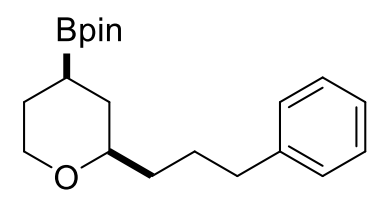
cis-4,4,5,5-tetramethyl-2-(2-(3-phenylpropyl)-tetrahydro-2H-pyran-4-yl)-1,3,2-dioxaborolane (4j). This compound was synthesized according to general procedure A. The residue was purified by column chromatography on silica gel to afford the product $\mathbf{4 j}$ ( $94.0 \mathrm{mg}, 71 \%$ yield, $\mathrm{dr}>20: 1$ ) as a colorless oil; ${ }^{1} \mathbf{H}$ NMR (600 MHz, Chloroform-d) $\delta 7.28$ - $7.23(\mathrm{~m}, 2 \mathrm{H}), 7.18$ - $7.14(\mathrm{~m}$, $3 \mathrm{H}), 3.98(\mathrm{ddd}, J=11.3,4.3,1.8 \mathrm{~Hz}, 1 \mathrm{H}), 3.39(\mathrm{td}, J=11.4,2.9 \mathrm{~Hz}, 1 \mathrm{H}), 3.22(\mathrm{dddd}, J=10.5$, 7.3, 5.2, $2.1 \mathrm{~Hz}, 1 \mathrm{H}), 2.60(\mathrm{t}, J=7.8 \mathrm{~Hz}, 2 \mathrm{H}), 1.79-1.70(\mathrm{~m}, 1 \mathrm{H}), 1.68-1.60(\mathrm{~m}, 2 \mathrm{H}), 1.56-$ 
$1.48(\mathrm{~m}, 3 \mathrm{H}), 1.46-1.39(\mathrm{~m}, 1 \mathrm{H}), 1.26-1.24(\mathrm{~m}, 1 \mathrm{H}), 1.23(\mathrm{~s}, 12 \mathrm{H}), 1.18$ - $1.11(\mathrm{~m}, 1 \mathrm{H}) \mathrm{ppm}$; ${ }^{13}$ C NMR (151 MHz, $\left.\mathbf{C D C l}_{3}\right) \delta 142.7,128.6,128.4,125.7,83.2,78.3,69.3,36.4,36.2,33.0$, 27.5, 27.4, 24.89, 24.86 ppm; ${ }^{11}$ B NMR (128 MHz, Chloroform-d) $\delta 32.64$ ppm; HRMS (ESI) calculated $[\mathrm{M}+\mathrm{H}]^{+}$for $\mathrm{C}_{20} \mathrm{H}_{32} \mathrm{BO}_{3}{ }^{+}=331.2439$, found: 331.2436 .

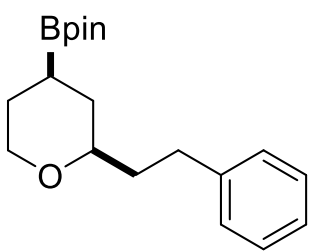

cis-4,4,5,5-tetramethyl-2-(2-phenethyltetrahydro-2H-pyran-4-yl)1,3,2-dioxaborolane (4k). This compound was synthesized according to general procedure A. The residue was purified by column chromatography on silica gel to afford the product $\mathbf{4 k}(83.9 \mathrm{mg}, 66 \%$ yield, $\mathrm{dr}>20: 1)$ as a colorless oil; ${ }^{1} \mathbf{H}$ NMR (400 MHz, Chloroform-d) $\delta 7.29-7.27$ (m, 1H), $7.26-7.24(\mathrm{~m}, 1 \mathrm{H}), 7.20-7.14(\mathrm{~m}, 3 \mathrm{H}), 4.03(\mathrm{dt}, J=11.2,3.4 \mathrm{~Hz}, 1 \mathrm{H}), 3.46-3.36(\mathrm{~m}, 1 \mathrm{H})$, $3.22(\mathrm{dddd}, J=10.3,7.8,4.7,2.1 \mathrm{~Hz}, 1 \mathrm{H}), 2.76(\mathrm{ddd}, J=13.7,10.0,5.5 \mathrm{~Hz}, 1 \mathrm{H}), 2.65$ (ddd, $J$ $=13.7,9.8,6.8 \mathrm{~Hz}, 1 \mathrm{H}), 1.80(\mathrm{dddd}, J=13.4,9.9,7.8,5.5 \mathrm{~Hz}, 1 \mathrm{H}), 1.73-1.67(\mathrm{~m}, 1 \mathrm{H}), 1.67$ - $1.61(\mathrm{~m}, 1 \mathrm{H}), 1.60-1.50(\mathrm{~m}, 2 \mathrm{H}), 1.29-1.25(\mathrm{~m}, 1 \mathrm{H}), 1.23(\mathrm{~s}, 12 \mathrm{H}), 1.19-1.12(\mathrm{~m}, 1 \mathrm{H})$ ppm; ${ }^{13}$ C NMR (101 MHz, $\left.\mathbf{C D C l}_{3}\right) \delta 142.6,128.6,128.4,125.7,83.2,77.6,69.3,38.4,32.9$, 31.8, 27.4, 24.9, 24.8 ppm; ${ }^{11}$ B NMR (128 MHz, Chloroform-d) $\delta 31.87$ ppm; HRMS (ESI) calculated $[\mathrm{M}+\mathrm{H}]^{+}$for $\mathrm{C}_{19} \mathrm{H}_{30} \mathrm{BO}_{3}{ }^{+}=317.2283$, found: 317.2281 .

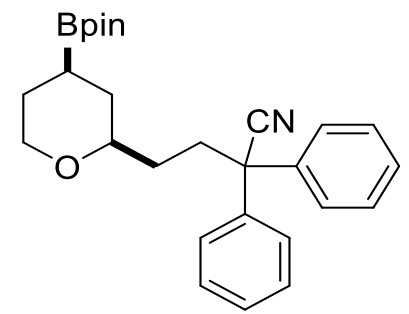

cis-2,2-diphenyl-4-(4-(4,4,5,5-tetramethyl-1,3,2-dioxaborolan2-yl)-tetrahydro-2H-pyran-2-yl)-butanenitrile (4l). This compound was synthesized according to general procedure A. The residue was purified by column chromatography on silica gel to afford the product $4 \mathbf{1}(76.0 \mathrm{mg}, 44 \%$ yield, $\mathrm{dr}>20: 1)$ as a colorless oil; ${ }^{1} \mathbf{H}$ NMR (400 MHz, Chloroform-d) $\delta 7.41-7.36(\mathrm{~m}, 4 \mathrm{H}), 7.36-7.31(\mathrm{~m}, 4 \mathrm{H}), 7.30-7.24(\mathrm{~m}, 2 \mathrm{H}), 3.97$ (ddd, $J=$ 11.3, 4.1, $1.9 \mathrm{~Hz}, 1 \mathrm{H}), 3.36(\mathrm{td}, J=11.2,3.2 \mathrm{~Hz}, 1 \mathrm{H}), 3.23(\mathrm{dddd}, J=10.2,7.0,5.5,2.0 \mathrm{~Hz}$, 1H), 2.64 (ddd, $J=13.6,9.3,7.3 \mathrm{~Hz}, 1 \mathrm{H}), 2.41-2.25(\mathrm{~m}, 1 \mathrm{H}), 1.63-1.54(\mathrm{~m}, 3 \mathrm{H}), 1.53-1.42$ $(\mathrm{m}, 2 \mathrm{H}), 1.27-1.23(\mathrm{~m}, 1 \mathrm{H}), 1.22(\mathrm{~s}, 12 \mathrm{H}), 1.18-1.12(\mathrm{~m}, 1 \mathrm{H}) \mathrm{ppm} ;{ }^{13} \mathbf{C}$ NMR (101 MHz, $\left.\mathbf{C D C l}_{3}\right) \delta 140.6,140.1,128.9,128.9,127.9,127.1,127.0,122.5,83.2,77.9,69.3,51.7,35.9$, 32.9, 32.7, 27.2, 24.85, 24.83 ppm; ${ }^{11}$ B NMR (128 MHz, Chloroform-d) $\delta 33.89$ ppm; HRMS 
(ESI) calculated $[\mathrm{M}+\mathrm{H}]^{+}$for $\mathrm{C}_{27} \mathrm{H}_{35} \mathrm{BNO}_{3}{ }^{+}=432.2705$, found: 432.2708 .

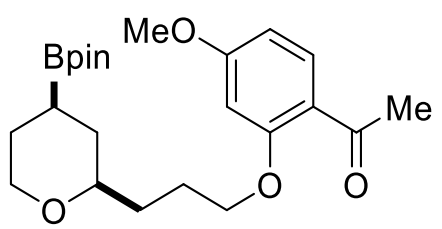

cis-1-(4-methoxy-2-(3-(4-(4,4,5,5-tetramethyl-1,3,2-dioxaborolan-2-yl)-tetrahydro-2H-pyran-2-yl)-propoxy)-phenyl)-ethan-1-one(4m). This compound was synthesized according to general procedure A. The residue was purified by column chromatography on silica gel to afford the product $\mathbf{4 m}(129.0 \mathrm{mg}, 77 \%$ yield, $\mathrm{dr}>20: 1)$ as a colorless oil; ${ }^{1} \mathrm{H}$ NMR $(400 \mathrm{MHz}$, Chloroform- $d) \delta 7.80(\mathrm{~d}, J=8.7 \mathrm{~Hz}, 1 \mathrm{H}), 6.46(\mathrm{dd}, J=8.7$, $2.3 \mathrm{~Hz}, 1 \mathrm{H}), 6.39(\mathrm{~d}, J=2.3 \mathrm{~Hz}, 1 \mathrm{H}), 4.04-3.94(\mathrm{~m}, 3 \mathrm{H}), 3.80(\mathrm{~s}, 3 \mathrm{H}), 3.38(\mathrm{td}, J=11.2,3.2$ $\mathrm{Hz}, 1 \mathrm{H}), 3.24(\mathrm{dtd}, J=10.6,6.2,2.1 \mathrm{~Hz}, 1 \mathrm{H}), 2.55(\mathrm{~s}, 3 \mathrm{H}), 2.02-1.81(\mathrm{~m}, 2 \mathrm{H}), 1.68-1.55(\mathrm{~m}$, 3H), $1.54-1.44(\mathrm{~m}, 2 \mathrm{H}), 1.26-1.22(\mathrm{~m}, 1 \mathrm{H}), 1.20(\mathrm{~s}, 12 \mathrm{H}), 1.17-1.11(\mathrm{~m}, 1 \mathrm{H}) \mathrm{ppm} ;{ }^{13} \mathbf{C}$ NMR $\left(\mathbf{1 0 1} \mathbf{M H z}, \mathbf{C D C l}_{3}\right) \delta 197.9,164.6,160.7,132.7,121.1,105.1,98.9,83.2,77.8,69.3,68.7,55.6$, 33.2, 33.0, 32.3, 27.3, 25.4, 24.82, 24.80 ppm; ${ }^{11}$ B NMR (128 MHz, Chloroform-d) $\delta 34.20$ ppm; HRMS (ESI) calculated $[\mathrm{M}+\mathrm{H}]^{+}$for $\mathrm{C}_{23} \mathrm{H}_{36} \mathrm{BO}_{6}{ }^{+}=419.2600$, found: 419.2600.<smiles>BrC1CCOC(CCC(c2ccccc2)c2ccccc2)C1</smiles>
cis-2-(2-(3,3-diphenylpropyl)tetrahydro-2H-pyran-4-yl)-4,4,5,5tetramethyl-1,3,2-dioxaborolane (4n). This compound was synthesized according to general procedure A. The residue was purified by column chromatography on silica gel to afford the product 4n (152.5 mg, 94\% yield, dr > 20:1) as a colorless oil; ${ }^{1} \mathbf{H}$ NMR (400 MHz, Chloroform- $d) \delta 7.28-7.26(\mathrm{~m}, 1 \mathrm{H}), 7.25-7.20(\mathrm{~m}, 7 \mathrm{H}), 7.17-7.11(\mathrm{~m}, 2 \mathrm{H}), 3.97(\mathrm{ddd}, J=$ 11.3, 4.0, 1.9 Hz, 1H), $3.85(\mathrm{t}, J=7.8 \mathrm{~Hz}, 1 \mathrm{H}), 3.37(\mathrm{td}, J=11.2,3.3 \mathrm{~Hz}, 1 \mathrm{H}), 3.25-3.17(\mathrm{~m}$, 1H), $2.25-2.14(\mathrm{~m}, 1 \mathrm{H}), 2.11-1.99(\mathrm{~m}, 1 \mathrm{H}), 1.61-1.40(\mathrm{~m}, 4 \mathrm{H}), 1.38-1.28(\mathrm{~m}, 1 \mathrm{H}), 1.25-$ $1.22(\mathrm{~m}, 1 \mathrm{H}), 1.21(\mathrm{~s}, 12 \mathrm{H}), 1.17-1.11(\mathrm{~m}, 1 \mathrm{H}) \mathrm{ppm} ;{ }^{13} \mathbf{C} \mathbf{~ N M R}\left(\mathbf{1 0 1} \mathbf{~ M H z}, \mathbf{C D C l}_{\mathbf{3}}\right) \delta$ 145.3, $145.1,128.5,128.0,126.1,83.2,78.4,69.3,51.6,35.3,33.0,31.6,27.4,24.85,24.83 \mathrm{ppm} ;{ }^{11} \mathbf{B}$ NMR (128 MHz, Chloroform-d) $\delta 34.71$ ppm; HRMS (ESI) calculated $[\mathrm{M}+\mathrm{H}]^{+}$for $\mathrm{C}_{26} \mathrm{H}_{36} \mathrm{BO}_{3}^{+}=407.2752$, found: 407.2752. 


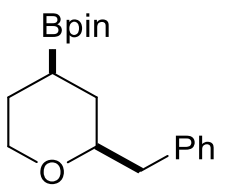

cis-2-(2-benzyltetrahydro-2H-pyran-4-yl)-4,4,5,5-tetramethyl-1,3,2dioxaborolane (40). This compound was synthesized according to general procedure A using benzyl chloride ( $0.6 \mathrm{mmol}, 1.5$ equiv). The residue was purified by column chromatography on silica gel to afford the product $4 \mathbf{0}(83.6 \mathrm{mg}, 69 \%$ yield, $\mathrm{dr}>$ 20:1) as a colorless oil; ${ }^{1} \mathbf{H}$ NMR (400 MHz, Chloroform- $\left.d\right) \delta$ $7.29-7.25(\mathrm{~m}, 2 \mathrm{H}), 7.22$ $7.16(\mathrm{~m}, 3 \mathrm{H}), 3.99(\mathrm{dt}, J=11.3,3.3 \mathrm{~Hz}, 1 \mathrm{H}), 3.50-3.33(\mathrm{~m}, 2 \mathrm{H}), 2.84(\mathrm{dd}, J=13.7,6.7 \mathrm{~Hz}$, 1H), $2.63(\mathrm{dd}, J=13.8,6.3 \mathrm{~Hz}, 1 \mathrm{H}), 1.68-1.65(\mathrm{~m}, 1 \mathrm{H}), 1.56-1.50(\mathrm{~m}, 2 \mathrm{H}), 1.30-1.27(\mathrm{~m}$, 1H), $1.21(\mathrm{~s}, 12 \mathrm{H}), 1.17-1.06(\mathrm{~m}, 1 \mathrm{H}) \mathrm{ppm} ;{ }^{13} \mathbf{C}$ NMR (101 MHz, $\left.\mathbf{C D C l}_{3}\right) \delta$ 139.0, 129.5, 128.3, 126.2, 83.2, 79.5, 69.5, 43.3, 32.5, 27.3, 24.8 ppm; ${ }^{11}$ B NMR (128 MHz, Chloroformd) $\delta 33.25$ ppm; HRMS (ESI) calculated $[\mathrm{M}+\mathrm{H}]^{+}$for $\mathrm{C}_{18} \mathrm{H}_{28} \mathrm{BO}_{3}{ }^{+}=303.2126$, found: 303.2123 .<smiles>COc1ccc(CC2CC(Br)CCO2)cc1</smiles>

cis-2-(2-(4-methoxybenzyl)-tetrahydro-2H-pyran-4-yl)-4,4,5,5tetramethyl-1,3,2-dioxaborolane (4p). This compound was synthesized according to general procedure A using 4Methoxybenzylchloride ( $0.6 \mathrm{mmol}, 1.5$ equiv). The residue was purified by column chromatography on silica gel to afford the product $4 \mathbf{p}(70.8 \mathrm{mg}, 53 \%$ yield, $\mathrm{dr}>20: 1)$ as a colorless oil; ${ }^{1}$ H NMR (400 MHz, Chloroform-d) $\delta 7.15$ - 7.09 (m, 2H), 6.85 - 6.79 (m, 2H), $4.00(\mathrm{dt}, J=11.3,3.3 \mathrm{~Hz}, 1 \mathrm{H}), 3.78(\mathrm{~s}, 3 \mathrm{H}), 3.46-3.32(\mathrm{~m}, 2 \mathrm{H}), 2.79(\mathrm{dd}, J=13.9,6.7 \mathrm{~Hz}$, 1H), $2.58(\mathrm{dd}, J=13.9,6.3 \mathrm{~Hz}, 1 \mathrm{H}), 1.65(\mathrm{dt}, J=12.0,2.5 \mathrm{~Hz}, 1 \mathrm{H}), 1.56-1.50(\mathrm{~m}, 2 \mathrm{H}), 1.30$ - $1.28(\mathrm{~m}, 1 \mathrm{H}), 1.22(\mathrm{~s}, 12 \mathrm{H}), 1.16-1.07(\mathrm{~m}, 1 \mathrm{H}) \mathrm{ppm} ;{ }^{13} \mathbf{C}$ NMR (101 MHz, Chloroform-d) $\delta 158.0,131.1,130.4,113.7,83.2,79.6,69.5,55.3,42.4,32.5,27.3,24.9$ ppm; ${ }^{11}$ B NMR (128 MHz, Chloroform-d) $\delta 33.70$ ppm; HRMS (ESI) calculated $[\mathrm{M}+\mathrm{H}]^{+}$for $\mathrm{C}_{19} \mathrm{H}_{30} \mathrm{BO}_{4}{ }^{+}=$ 333.2232, found: 333.2233 .<smiles>CC(C)CC1CC(Br)CCO1</smiles>
cis-2-(2-isobutyltetrahydro-2H-pyran-4-yl)-4,4,5,5-tetramethyl-1,3,2dioxaborolane (4q). This compound was synthesized according to general procedure A. The residue was purified by column chromatography on silica gel to afford the product $\mathbf{4 q}(65.8 \mathrm{mg}, 61 \%$ yield, $\mathrm{dr}>20: 1)$ as a colorless oil; ${ }^{1} \mathbf{H}$ NMR (400 
MHz, Chloroform- $d$ ) $\delta 3.96(\mathrm{ddd}, J=11.3,3.9,2.3 \mathrm{~Hz}, 1 \mathrm{H}), 3.43-3.33(\mathrm{~m}, 1 \mathrm{H}), 3.25$ (dddd, $J=10.1,8.0,4.9,2.1 \mathrm{~Hz}, 1 \mathrm{H}), 1.80-1.69(\mathrm{~m}, 1 \mathrm{H}), 1.62-1.57(\mathrm{~m}, 1 \mathrm{H}), 1.56-1.46(\mathrm{~m}, 2 \mathrm{H})$, $1.40(\mathrm{ddd}, \mathrm{J}=14.1,8.0,6.2 \mathrm{~Hz}, 1 \mathrm{H}), 1.22(\mathrm{~s}, 12 \mathrm{H}), 1.19-1.10(\mathrm{~m}, 3 \mathrm{H}), 0.86(\mathrm{~d}, J=6.6 \mathrm{~Hz}$, 6H) ppm; ${ }^{13} \mathbf{C}$ NMR (101 MHz, $\left.\mathbf{C D C l}_{3}\right) \delta$ 83.2, 76.6, 69.3, 46.0, 33.4, 27.5, 24.8, 24.3, 23.3, 22.6 ppm; ${ }^{11}$ B NMR (128 MHz, Chloroform-d) $\delta 33.00$ ppm; HRMS (ESI) calculated $[\mathrm{M}+\mathrm{H}]^{+}$ for $\mathrm{C}_{15} \mathrm{H}_{30} \mathrm{BO}_{3}{ }^{+}=269.2283$, found: 269.2284 .<smiles>Brc1ccccc1</smiles>

cis-4,4,5,5-tetramethyl-2-(octahydro-2H,2'H-[2,4'-bipyran]-4-yl)-1,3,2dioxaborolane (4r). This compound was synthesized according to general procedure A. The residue was purified by column chromatography on silica gel to afford the product $4 \mathbf{r}(47.1 \mathrm{mg}, 40 \%$ yield, $\mathrm{dr}>20: 1)$ as a colorless oil; ${ }^{1}$ H NMR (400 MHz, Chloroform-d) $\delta 4.06$ - 3.90 (m, 3H), 3.46 - 3.26 (m, 3H), 2.97 (ddd, $J$ $=10.5,6.9,2.1 \mathrm{~Hz}, 1 \mathrm{H}), 1.79(\mathrm{ddt}, J=11.2,3.7,2.0 \mathrm{~Hz}, 1 \mathrm{H}), 1.69(\mathrm{dq}, J=12.4,2.0 \mathrm{~Hz}, 1 \mathrm{H})$, $1.60-1.47(\mathrm{~m}, 4 \mathrm{H}), 1.42-1.31(\mathrm{~m}, 2 \mathrm{H}), 1.24(\mathrm{~s}, 12 \mathrm{H}), 1.20-1.08(\mathrm{~m}, 2 \mathrm{H}) \mathrm{ppm} ;{ }^{13} \mathbf{C}$ NMR $\left(\mathbf{1 0 1} \mathbf{~ M H z}, \mathbf{C D C l}_{\mathbf{3}}\right) \delta 83.2,82.1,69.5,68.3,68.0,40.9,29.7,29.5,28.8,27.5,24.9 \mathrm{ppm} ;{ }^{11} \mathbf{B}$ NMR (128 MHz, Chloroform-d) $\delta 32.51$ ppm; HRMS (ESI) calculated $[\mathrm{M}+\mathrm{H}]^{+}$for $\mathrm{C}_{16} \mathrm{H}_{30} \mathrm{BO}_{4}{ }^{+}=297.2232$, found: 297.2234 .

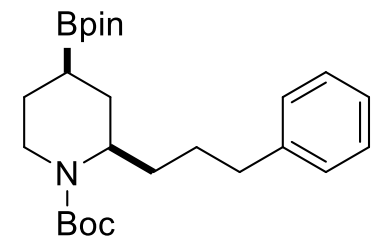

cis-tert-butyl-2-(3-phenylpropyl)-4-(4,4,5,5-tetramethyl-1,3,2-dioxaborolan-2-yl)-piperidine-1-carboxylate (4s). This compound was synthesized according to general procedure A. The residue was purified by column chromatography on silica gel to afford the product 4s (116.0 $\mathrm{mg}, 68 \%$ yield, $\mathrm{dr}>20: 1)$ as a colorless oil; ${ }^{1} \mathbf{H}$ NMR (600 $\mathbf{M H z}$, Chloroform-d) $\delta 7.29$ - $7.22(\mathrm{~m}, 2 \mathrm{H}), 7.19-7.12(\mathrm{~m}, 3 \mathrm{H}), 4.13-4.06(\mathrm{~m}, 1 \mathrm{H}), 3.87$ (ddd, $J=$ 13.7, 5.4, $2.5 \mathrm{~Hz}, 1 \mathrm{H}), 2.80(\mathrm{ddd}, J=13.6,12.3,3.8 \mathrm{~Hz}, 1 \mathrm{H}), 2.68-2.49(\mathrm{~m}, 2 \mathrm{H}), 1.82(\mathrm{dt}, J=$ 13.5, $6.0 \mathrm{~Hz}, 1 \mathrm{H}), 1.73-1.55(\mathrm{~m}, 6 \mathrm{H}), 1.49-1.44(\mathrm{~m}, 1 \mathrm{H}), 1.43$ (s, 9H), $1.23(\mathrm{~d}, J=3.3 \mathrm{~Hz}$, 12H), 1.20 - 1.16 (m, 1H) ppm; ${ }^{13}$ C NMR (151 MHz, Chloroform-d) $\delta$ 155.4, 142.6, 128.4, $128.4,125.8,83.3,79.0,51.8,37.9,35.8,31.8,29.1,28.6,28.2,25.8,25.1,24.9$ ppm; ${ }^{11} \mathbf{B}$ NMR (128 MHz, Chloroform-d) $\delta 36.86$ ppm; HRMS (ESI) calculated $[\mathrm{M}+\mathrm{H}]^{+}$for $\mathrm{C}_{25} \mathrm{H}_{41} \mathrm{BNO}_{4}{ }^{+}=$ 
430.3123, found: 430.3124 .

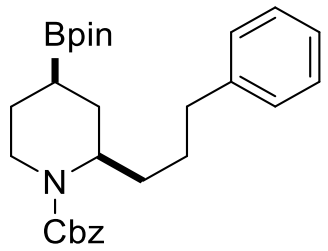

cis-benzyl-2-(3-phenylpropyl)-4-(4,4,5,5-tetramethyl-1,3,2-dioxaborolan-2-yl)-piperidine-1-carboxylate $(\mathbf{4 t})$. This compound was synthesized according to general procedure A. The residue was purified by column chromatography on silica gel to afford the product $\mathbf{4 t}$ (130.2 mg, 68\% yield, $\mathrm{dr}>20: 1)$ as a colorless oil; ${ }^{1} \mathbf{H}$ NMR (400 MHz, Chloroform- $d$ ) $\delta 7.34-7.22$ (m, 7H), $7.17-7.09(\mathrm{~m}, 3 \mathrm{H}), 5.11(\mathrm{~d}, J=2.0 \mathrm{~Hz}, 2 \mathrm{H}), 4.19(\mathrm{dq}, J=11.6,6.1,4.8 \mathrm{~Hz}, 1 \mathrm{H}), 3.95$ (ddd, $J=13.6,5.3,2.5 \mathrm{~Hz}, 1 \mathrm{H}), 2.89$ (ddd, $J=13.7,12.4,3.7 \mathrm{~Hz}, 1 \mathrm{H}), 2.63-2.51(\mathrm{~m}, 2 \mathrm{H})$, $1.88-1.80(\mathrm{~m}, 1 \mathrm{H}), 1.77-1.61(\mathrm{~m}, 5 \mathrm{H}), 1.60-1.54(\mathrm{~m}, 1 \mathrm{H}), 1.46(\mathrm{dtd}, J=11.1,7.8,7.3,3.1$ $\mathrm{Hz}, 1 \mathrm{H}), 1.22(\mathrm{~d}, J=2.4 \mathrm{~Hz}, 12 \mathrm{H}), 1.20-1.15(\mathrm{~m}, 1 \mathrm{H}) \mathrm{ppm} ;{ }^{13} \mathbf{C} \mathbf{N M R}\left(\mathbf{1 0 1} \mathbf{M H z}, \mathbf{C D C l}_{3}\right) \delta$ $155.8,142.4,137.2,128.5,128.39,128.35,127.9,127.83,125.76,83.3,66.9,52.1,38.3,35.8$, 31.7, 29.2, 28.2, 25.8, 25.0, 24.8 ppm; ${ }^{11}$ B NMR (128 MHz, Chloroform-d) $\delta 29.40$ ppm; HRMS (ESI) calculated $[\mathrm{M}+\mathrm{H}]^{+}$for $\mathrm{C}_{28} \mathrm{H}_{39} \mathrm{BNO}_{4}{ }^{+}=464.2967$, found: 464.2968 .

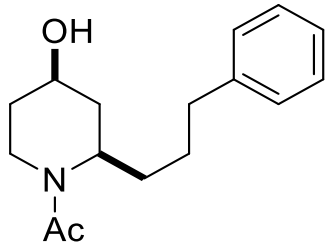

cis - 1- (4-hydroxy-2-(3-phenylpropyl)-piperidin-1-yl) - ethan-1-one (4u). This compound was synthesized according to general procedure A. The residue was purified by column chromatography on silica gel to afford the product $\mathbf{4 u}(66.0 \mathrm{mg}, 63 \%$ yield, $\mathrm{dr}>20: 1)$ as a colorless oil;

${ }^{1}$ H NMR (400 MHz, DMSO- $\left.\boldsymbol{d}_{\mathbf{6}} \mathbf{1 0 0}^{\circ} \mathrm{C}\right) \delta 7.34-7.26(\mathrm{~m}, 2 \mathrm{H}), 7.26-7.17(\mathrm{~m}, 3 \mathrm{H}), 4.28(\mathrm{~s}$, 1H), $4.01-3.97(\mathrm{~m}, 1 \mathrm{H}), 3.81(\mathrm{~s}, 1 \mathrm{H}), 3.21(\mathrm{~s}, 1 \mathrm{H}), 2.69-2.60(\mathrm{~m}, 2 \mathrm{H}), 2.02(\mathrm{~s}, 3 \mathrm{H}), 1.96(\mathrm{td}$, $J=8.8,5.2 \mathrm{~Hz}, 1 \mathrm{H}), 1.85-1.74(\mathrm{~m}, 1 \mathrm{H}), 1.72-1.68(\mathrm{~m}, 2 \mathrm{H}), 1.68-1.59(\mathrm{~m}, 3 \mathrm{H}), 1.59-1.52$ (m, 1H) ppm; ${ }^{13} \mathbf{C}$ NMR (101 MHz, DMSO-D $\left.\mathbf{6}_{\mathbf{6}} \mathbf{1 0 0}^{\circ} \mathbf{C}\right) \delta 168.5,142.9,128.7,128.6,126.0$, 63.5, 35.6, 35.1, 33.2, 32.9, 28.6, 21.8 ppm; HRMS (ESI) calculated $[\mathrm{M}+\mathrm{H}]^{+}$for $\mathrm{C}_{16} \mathrm{H}_{24} \mathrm{NO}_{2}{ }^{+}$ $=262.1802$, found: 262.1801 .

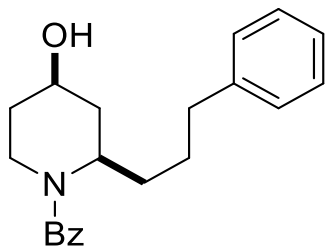

cis-(4-hydroxy-2-(3-phenylpropyl)-piperidin-1-yl)-(phenyl)methanone (4v). This compound was synthesized according to general procedure A. The residue was purified by column chromatography on silica gel to afford the product $4 \mathbf{v}(56.4 \mathrm{mg}, 44 \%$ yield, $\mathrm{dr}>20: 1)$ as a 
colorless oil; ${ }^{1} \mathbf{H}$ NMR $(\mathbf{6 0 0} \mathbf{~ M H z}$, Chloroform- $\boldsymbol{d}) \delta 7.38(\mathrm{dd}, J=5.4,2.0 \mathrm{~Hz}, 3 \mathrm{H}), 7.34-7.30$ (m, 2H), 7.28 - $7.27(\mathrm{~m}, 1 \mathrm{H}), 7.27$ - $7.24(\mathrm{~m}, 1 \mathrm{H}), 7.19-7.14(\mathrm{~m}, 3 \mathrm{H}), 4.20(\mathrm{p}, J=2.7 \mathrm{~Hz}, 1 \mathrm{H})$, $3.36(\mathrm{~s}, 2 \mathrm{H}), 2.60(\mathrm{~s}, 2 \mathrm{H}), 2.16-2.06(\mathrm{~m}, 1 \mathrm{H}), 1.88-1.83(\mathrm{~m}, 1 \mathrm{H}), 1.81-1.73(\mathrm{~m}, 3 \mathrm{H}), 1.68$ 1.56 (m, 5H) ppm; ${ }^{13} \mathbf{C}$ NMR (151 MHz, $\left.\mathbf{C D C l}_{3}\right) \delta 170.9,142.4,137.0,129.4,128.6,128.5$, 128.4, 126.6, 125.8, 64.9, 35.7, 34.9, 33.2, 32.6, 28.7, 25.0 ppm; HRMS (ESI) calculated $[\mathrm{M}+\mathrm{H}]^{+}$for $\mathrm{C}_{21} \mathrm{H}_{26} \mathrm{NO}_{2}{ }^{+}=324.1958$, found: 324.1956 .

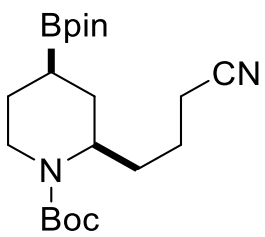

cis-tert-butyl-2-(3-cyanopropyl)-4-(4,4,5,5-tetramethyl-1,3,2-dioxaborolan-2-yl)-piperidine-1-carboxylate (4z). This compound was synthesized according to general procedure A. The residue was purified by column chromatography on silica gel to afford the product $\mathbf{4 z}(103.0 \mathrm{mg}$, $68 \%$ yield, $\mathrm{dr}>20: 1)$ as a white solid; ${ }^{1} \mathbf{H}$ NMR (600 MHz, Chloroform-d) $\delta 4.10$ (dq, $J=$ $10.2,5.3 \mathrm{~Hz}, 1 \mathrm{H}), 3.92-3.83(\mathrm{~m}, 1 \mathrm{H}), 2.79(\mathrm{ddd}, J=13.7,12.2,3.8 \mathrm{~Hz}, 1 \mathrm{H}), 2.38(\mathrm{t}, J=7.0$ $\mathrm{Hz}, 2 \mathrm{H}), 1.85(\mathrm{dt}, J=13.6,6.2 \mathrm{~Hz}, 1 \mathrm{H}), 1.81-1.69(\mathrm{~m}, 2 \mathrm{H}), 1.69-1.58(\mathrm{~m}, 4 \mathrm{H}), 1.56-1.50$ (m, 1H), 1.45 (s, 9H), $1.26(\mathrm{~s}, 12 \mathrm{H}), 1.23$ - $1.18(\mathrm{~m}, 1 \mathrm{H}) \mathrm{ppm} ;{ }^{13} \mathbf{C}$ NMR (151 MHz, $\left.\mathbf{C D C l}_{3}\right) \delta$ $155.3,119.7,83.4,79.3,50.76,37.9,31.1,29.2,28.5,25.5,25.0,24.8,22.5,16.9$ ppm; ${ }^{11} \mathbf{B}$ NMR (128 MHz, Chloroform-d) $\delta 33.00$ ppm; HRMS (ESI) calculated $[\mathrm{M}+\mathrm{H}]^{+}$for $\mathrm{C}_{20} \mathrm{H}_{36} \mathrm{BN}_{2} \mathrm{O}_{4}{ }^{+}=379.2763$, found: 379.2759 .<smiles>O=C(OCCCC1CC(Br)CCN1C(=O)OCc1ccccc1)C(c1ccccc1)c1ccccc1</smiles>
cis-benzyl-2-(3-(2,2-diphenylacetoxy)-propyl)-4-(4,4,5,5-tetramethyl-1,3,2-dioxaborolan-2-yl)-piperidine-1-carboxylate (4aa). This compound was synthesized according to general procedure A. The residue was purified by column chromatography on silica gel to afford the product $\mathbf{4 a a}(155.0 \mathrm{mg}, 65 \%$ yield, $\mathrm{dr}>20: 1)$ as a colorless oil; ${ }^{1} \mathbf{H}$ NMR (400 MHz, Chloroform-d) $\delta 7.33$ - 7.28 (m, 13H), 7.27 - $7.22(\mathrm{~m}, 2 \mathrm{H}), 5.10(\mathrm{~s}, 2 \mathrm{H})$, $4.99(\mathrm{~s}, 1 \mathrm{H}), 4.16-4.06(\mathrm{~m}, 3 \mathrm{H}), 3.99-3.80(\mathrm{~m}, 1 \mathrm{H}), 2.88-2.79(\mathrm{~m}, 1 \mathrm{H}), 1.81(\mathrm{dt}, J=13.5$, $5.9 \mathrm{~Hz}, 1 \mathrm{H}), 1.75$ - $1.68(\mathrm{~m}, 1 \mathrm{H}), 1.66-1.57(\mathrm{~m}, 4 \mathrm{H}), 1.56-1.48(\mathrm{~m}, 1 \mathrm{H}), 1.44$ - $1.33(\mathrm{~m}, 1 \mathrm{H})$, $1.23-1.22(\mathrm{~m}, 1 \mathrm{H}), 1.20(\mathrm{~d}, J=6.2 \mathrm{~Hz}, 12 \mathrm{H}) \mathrm{ppm} ;{ }^{13} \mathbf{C} \mathbf{N M R}\left(\mathbf{1 0 1} \mathbf{M H z}, \mathbf{C D C l}_{3}\right) \delta 172.5$, 
$155.7,138.7,137.0,128.6,128.5,127.9,127.8,127.3,83.3,66.9,65.1,60.4,57.1,51.8,38.1$, 29.1, 28.4, 25.6, 24.9, 24.7 ppm; ${ }^{11}$ B NMR (128 MHz, Chloroform-d) $\delta 31.41$ ppm; HRMS (ESI) calculated $[\mathrm{M}+\mathrm{H}]^{+}$for $\mathrm{C}_{36} \mathrm{H}_{45} \mathrm{BNO}_{6}{ }^{+}=598.3335$, found: 598.3332 .

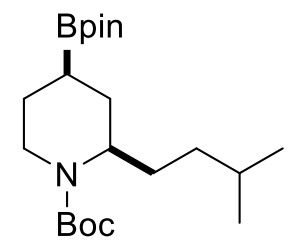

cis-tert-butyl-2-isopentyl-4-(4,4,5,5-tetramethyl-1,3,2-dioxaborolan-2yl)-piperidine-1-carboxylate (4ab). This compound was synthesized according to general procedure A. The residue was purified by column chromatography on silica gel to afford the product $\mathbf{4 a b}(125.0 \mathrm{mg}, 82 \%$ yield, dr > 20:1) as a colorless oil; ${ }^{1} \mathbf{H}$ NMR (400 MHz, Chloroform-d) $\delta 4.02$ (dtd, $J=9.7$, 6.0, $3.8 \mathrm{~Hz}, 1 \mathrm{H}), 3.88(\mathrm{ddd}, J=13.7,5.4,2.5 \mathrm{~Hz}, 1 \mathrm{H}), 2.81(\mathrm{ddd}, J=13.6,12.3,3.8 \mathrm{~Hz}, 1 \mathrm{H})$, $1.83(\mathrm{dt}, J=13.6,6.0 \mathrm{~Hz}, 1 \mathrm{H}), 1.72(\mathrm{dd}, J=13.0,3.5 \mathrm{~Hz}, 1 \mathrm{H}), 1.68-1.62(\mathrm{~m}, 1 \mathrm{H}), 1.61-1.57$ (m, 1H), $1.55-1.48(\mathrm{~m}, 2 \mathrm{H}), 1.45(\mathrm{~s}, 9 \mathrm{H}), 1.40-1.33(\mathrm{~m}, 1 \mathrm{H}), 1.25(\mathrm{~s}, 12 \mathrm{H}), 1.22-1.16(\mathrm{~m}$, 2H), $1.15-1.07(\mathrm{~m}, 1 \mathrm{H}), 0.87(\mathrm{dd}, J=6.6,3.7 \mathrm{~Hz}, 6 \mathrm{H}) \mathrm{ppm} ;{ }^{13} \mathbf{C} \mathbf{N M R}\left(\mathbf{1 5 1} \mathbf{~ M H z}, \mathbf{C D C l}_{3}\right) \delta$ $155.4,83.3,78.9,52.1,37.6,35.8,30.0,29.4,28.6,28.0,25.8,25.0,24.8,22.81,22.78$ ppm; ${ }^{11}$ B NMR (128 MHz, Chloroform-d) $\delta 29.46$ ppm; HRMS (ESI) calculated $[\mathrm{M}+\mathrm{H}]^{+}$for $\mathrm{C}_{21} \mathrm{H}_{41} \mathrm{BNO}_{4}{ }^{+}=382.3123$, found: 382.3117 .

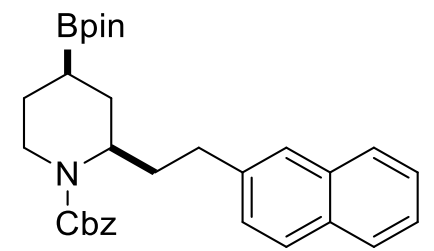

cis-benzyl-2-(2-(naphthalen-2-yl)-ethyl)-4-(4,4,5,5-tetramethyl-1,3,2-dioxaborolan-2-yl)-piperidine-1-carboxylate (4ac). This compound was synthesized according to general procedure A. The residue was purified by column chromatography on silica gel to afford the product $4 \mathbf{4 a c}(126.0 \mathrm{mg}, 63 \%$ yield, $\mathrm{dr}>20: 1)$ as a colorless oil; ${ }^{1} \mathbf{H}$ NMR (400 MHz, Chloroform-d) $\delta 7.98$ - $7.94(\mathrm{~m}, 1 \mathrm{H}), 7.87-7.80(\mathrm{~m}, 1 \mathrm{H}), 7.72$ - $7.64(\mathrm{~m}, 1 \mathrm{H}), 7.50$ $7.41(\mathrm{~m}, 2 \mathrm{H}), 7.38-7.32(\mathrm{~m}, 5 \mathrm{H}), 7.32-7.27(\mathrm{~m}, 2 \mathrm{H}), 5.17(\mathrm{~s}, 2 \mathrm{H}), 4.39$ (dq, J = 9.5, 4.8 Hz, 1H), $4.09(\mathrm{dd}, J=10.6,6.9 \mathrm{~Hz}, 1 \mathrm{H}), 3.10-2.98(\mathrm{~m}, 3 \mathrm{H}), 2.18-2.04(\mathrm{~m}, 1 \mathrm{H}), 1.94(\mathrm{dt}, J=13.6$, $6.0 \mathrm{~Hz}, 1 \mathrm{H}), 1.86-1.74(\mathrm{~m}, 3 \mathrm{H}), 1.69-1.61(\mathrm{~m}, 1 \mathrm{H}), 1.28-1.23(\mathrm{~m}, 1 \mathrm{H}), 1.01(\mathrm{~d}, J=13.9 \mathrm{~Hz}$, 12H) ppm; ${ }^{13} \mathbf{C}$ NMR (101 MHz, $\left.\mathbf{C D C l}_{3}\right) \delta$ 156.0, 138.5, 137.2, 133.9, 131.8, 128.8, 128.6, $128.0,127.9,126.6,125.9,125.7,125.6,125.5,123.8,83.4,67.1,52.4,38.5,33.6,29.91,29.88$, 
25.9, 24.8, 24.6 ppm; ${ }^{11}$ B NMR (128 MHz, Chloroform-d) $\delta 32.36$ ppm; HRMS (ESI) calculated $[\mathrm{M}+\mathrm{H}]^{+}$for $\mathrm{C}_{31} \mathrm{H}_{39} \mathrm{BNO}_{4}{ }^{+}=500.2967$, found: 500.2968 .

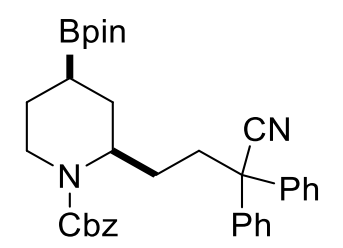

cis-benzyl-2-(2-(naphthalen-2-yl)-ethyl)-4-(4,4,5,5-tetramethyl-1,3,2 -dioxaborolan-2-yl)-piperidine-1-carboxylate (4ad). This compound was synthesized according to general procedure A. The residue was purified by column chromatography on silica gel to afford the product 4ad (117.0 mg, 52\% yield, dr > 20:1) as a white solid; ${ }^{1} \mathbf{H}$ NMR (400 MHz, Chloroform- $\left.d\right) \delta$ 7.38 - $7.31(\mathrm{~m}, 7 \mathrm{H}), 7.30$ - $7.26(\mathrm{~m}, 8 \mathrm{H}), 5.13(\mathrm{~s}, 2 \mathrm{H}), 4.26(\mathrm{dt}, J=13.9,4.6 \mathrm{~Hz}, 1 \mathrm{H}), 4.06$ $3.88(\mathrm{~m}, 1 \mathrm{H}), 2.80(\mathrm{td}, J=13.1,3.5 \mathrm{~Hz}, 1 \mathrm{H}), 2.35(\mathrm{dd}, J=9.6,6.8 \mathrm{~Hz}, 2 \mathrm{H}), 1.92-1.81(\mathrm{~m}$, 2H), $1.76-1.63(\mathrm{~m}, 2 \mathrm{H}), 1.62-1.49(\mathrm{~m}, 2 \mathrm{H}), 1.23-1.16(\mathrm{~m}, 1 \mathrm{H}), 1.10(\mathrm{~d}, J=4.8 \mathrm{~Hz}, 12 \mathrm{H})$ ppm; ${ }^{13} \mathbf{C}$ NMR (101 MHz, $\left.\mathbf{C D C l}_{3}\right) \delta$ 156.0, 140.6, 139.5, 137.1, 129.1, 129.0, 128.6, 128.04, $128.01,127.90,127.85,126.9,126.7,122.5,83.5,67.1,52.2,51.7,38.3,37.3,30.3,28.5,25.8$, 24.93, 24.87 ppm; ${ }^{11}$ B NMR (128 MHz, Chloroform- $d$ ) $\delta 34.73$ ppm; HRMS (ESI) calculated $[\mathrm{M}+\mathrm{H}]^{+}$for $\mathrm{C}_{35} \mathrm{H}_{42} \mathrm{BN}_{2} \mathrm{O}_{4}{ }^{+}=565.3232$, found: 565.3236 .

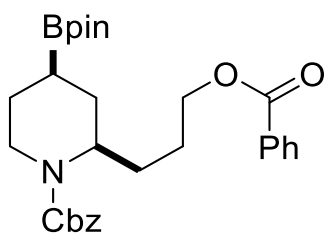

cis-benzyl-2-(3-(benzoyloxy)-propyl)-4-(4,4,5,5-tetramethyl-1,3,2dioxaborolan-2-yl)-piperidine-1-carboxylate (4ae). This compound was synthesized according to general procedure A. The residue was purified by column chromatography on silica gel to afford the product 4ae (164.0 mg, 81\% yield, dr > 20:1) as a colorless oil; ${ }^{1}$ H NMR (400 MHz, Chloroform- $d$ ) $\delta 8.05-8.00(\mathrm{~m}, 2 \mathrm{H}), 7.58$ - $7.52(\mathrm{~m}, 1 \mathrm{H}), 7.46-7.39(\mathrm{~m}, 2 \mathrm{H}), 7.36-7.31(\mathrm{~m}, 4 \mathrm{H}), 7.31-7.27$ (m, 1H), $5.13(\mathrm{~d}, J=3.5 \mathrm{~Hz}, 2 \mathrm{H}), 4.28(\mathrm{t}, J=6.1 \mathrm{~Hz}, 2 \mathrm{H}), 4.26-4.20(\mathrm{~m}, 1 \mathrm{H}), 4.03-3.93(\mathrm{~m}$, 1H), $2.93(\mathrm{ddd}, J=13.7,12.3,3.6 \mathrm{~Hz}, 1 \mathrm{H}), 1.92-1.85(\mathrm{~m}, 1 \mathrm{H}), 1.82-1.73(\mathrm{~m}, 4 \mathrm{H}), 1.73$ $1.68(\mathrm{~m}, 1 \mathrm{H}), 1.67-1.61(\mathrm{~m}, 1 \mathrm{H}), 1.59-1.51(\mathrm{~m}, 1 \mathrm{H}), 1.23-1.22(\mathrm{~m}, 1 \mathrm{H}), 1.20(\mathrm{~d}, J=1.6 \mathrm{~Hz}$, 12H) ppm; ${ }^{13} \mathbf{C}$ NMR (101 MHz, $\left.\mathbf{C D C l}_{3}\right) \delta 166.6,155.8,137.1,132.9,130.4,129.6,128.5$, $128.4,127.9,127.8,83.4,66.9,65.0,51.9,38.2,29.4,28.6,25.9,25.7,24.9,24.80 \mathrm{ppm} ;{ }^{11} \mathbf{B}$ NMR (128 MHz, Chloroform-d) $\delta 31.20$ ppm; HRMS (ESI) calculated $[\mathrm{M}+\mathrm{H}]^{+}$for $\mathrm{C}_{29} \mathrm{H}_{39} \mathrm{BNO}_{6}{ }^{+}=508.2865$, found: 508.2863 . 
<smiles>O=C(c1ccccc1)N1CCC(Br)CC1CCc1ccc2c(c1)CCO2</smiles>

cis-benzyl-2-(2-(2,3-dihydrobenzofuran-5-yl)-ethyl)-4-(4,4,5,5tetramethyl-1,3,2dioxaborolan-2-yl)-piperidine-1-carboxylate (4af). This compound was synthesized according to general procedure A. The residue was purified by column chromatography on silica gel to afford the product 4af $(138.0 \mathrm{mg}, 70 \%$ yield, $\mathrm{dr}>20: 1)$ as a colorless oil; ${ }^{1} \mathbf{H}$ NMR (400 MHz, Chloroform-d) $\delta 7.37-7.32(\mathrm{~m}, 4 \mathrm{H}), 7.32-7.27$ (m, 1H), 6.97 (d, $J=1.9$ $\mathrm{Hz}, 1 \mathrm{H}), 6.86(\mathrm{dd}, J=8.1,1.9 \mathrm{~Hz}, 1 \mathrm{H}), 6.64(\mathrm{~d}, J=8.1 \mathrm{~Hz}, 1 \mathrm{H}), 5.13(\mathrm{~d}, J=2.5 \mathrm{~Hz}, 2 \mathrm{H}), 4.50$ $(\mathrm{t}, J=8.7 \mathrm{~Hz}, 2 \mathrm{H}), 4.20(\mathrm{dtd}, J=9.4,5.9,3.6 \mathrm{~Hz}, 1 \mathrm{H}), 4.01-3.95(\mathrm{~m}, 1 \mathrm{H}), 3.11(\mathrm{t}, J=8.7 \mathrm{~Hz}$, 2H), $2.96(\mathrm{ddd}, J=13.6,12.2,3.6 \mathrm{~Hz}, 1 \mathrm{H}), 2.58-2.44(\mathrm{~m}, 2 \mathrm{H}), 1.96-1.85(\mathrm{~m}, 2 \mathrm{H}), 1.80$ $1.69(\mathrm{~m}, 2 \mathrm{H}), 1.68-1.59(\mathrm{~m}, 2 \mathrm{H}), 1.23-1.22(\mathrm{~m}, 1 \mathrm{H}), 1.17(\mathrm{~d}, J=6.9 \mathrm{~Hz}, 12 \mathrm{H}) \mathrm{ppm} ;{ }^{13} \mathbf{C}$ NMR (101 MHz, $\left.\mathbf{C D C l}_{3}\right) \delta 158.2,155.9,137.2,134.2,128.5,127.9,127.8,127.7,126.9,124.9$, 108.9, 83.4, 71.2, 66.9, 51.9, 38.4, 34.5, 32.1, 29.9, 29.3, 25.8, 25.0, 24.8 ppm; ${ }^{11}$ B NMR (128 MHz, Chloroform- $\boldsymbol{d}$ ) $\delta 35.13$ ppm; HRMS (ESI) calculated $[\mathrm{M}+\mathrm{H}]^{+}$for $\mathrm{C}_{29} \mathrm{H}_{39} \mathrm{BNO}_{5}{ }^{+}=$ 492.2916, found: 492.2914

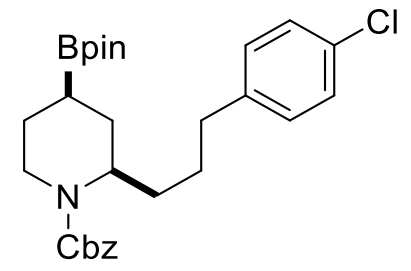

cis-benzyl-2-(3-(4-chlorophenyl)-propyl)-4-(4,4,5,5-tetramethyl-1,3,2-dioxaborolan-2-yl)-piperidine-1-carboxylate (4ag). This compound was synthesized according to general procedure A. The residue was purified by column chromatography on silica gel to afford the product $\mathbf{4 a g}(102.8 \mathrm{mg}, 52 \%$ yield, $\mathrm{dr}>20: 1)$ as a colorless oil; ${ }^{1} \mathbf{H}$ NMR (400 MHz, Chloroform- $d$ ) $\delta 7.34-7.28(\mathrm{~m}, 5 \mathrm{H}), 7.22-7.18(\mathrm{~m}, 2 \mathrm{H}), 7.03(\mathrm{~d}, J=8.4 \mathrm{~Hz}, 2 \mathrm{H}), 5.11$ $(\mathrm{d}, J=2.8 \mathrm{~Hz}, 2 \mathrm{H}), 4.18(\mathrm{q}, J=5.8 \mathrm{~Hz}, 1 \mathrm{H}), 4.00-3.90(\mathrm{~m}, 1 \mathrm{H}), 2.87(\mathrm{ddd}, J=13.7,12.3,3.7$ $\mathrm{Hz}, 1 \mathrm{H}), 2.61-2.46(\mathrm{~m}, 2 \mathrm{H}), 1.84(\mathrm{dt}, J=13.6,6.0 \mathrm{~Hz}, 1 \mathrm{H}), 1.77-1.71(\mathrm{~m}, 1 \mathrm{H}), 1.66-1.49$ $(\mathrm{m}, 5 \mathrm{H}), 1.47-1.38(\mathrm{~m}, 1 \mathrm{H}), 1.23-1.20(\mathrm{~m}, 13 \mathrm{H}) \mathrm{ppm} ;{ }^{13} \mathbf{C} \mathbf{~ N M R}\left(\mathbf{1 0 1} \mathbf{~ M H z}, \mathbf{C D C l}_{\mathbf{3}}\right) \delta 155.8$, $140.9,137.2,131.5,129.8,128.54,128.46,128.0,127.9,83.4,66.9,52.0,38.3,35.1,31.6,29.2$, 28.1, 25.8, 25.0, 24.9 ppm; ${ }^{11}$ B NMR (128 MHz, Chloroform-d) $\delta 35.92$ ppm; HRMS (ESI) calculated $[\mathrm{M}+\mathrm{H}]^{+}$for $\mathrm{C}_{28} \mathrm{H}_{38} \mathrm{BClNO}_{4}{ }^{+}=498.2577$, found: 498.2580 . 


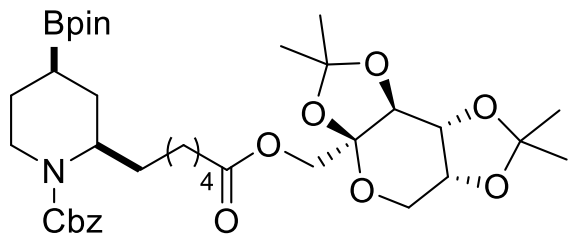

cis-benzyl-2-(6-oxo-6-(((3aS,5aR,8aR,8bS)-2,2,7,7tetramethyltetrahydro-3aH-bis-([1,3]-dioxolo)-[4,5b:4',5'-d]-pyran-3a-yl)-methoxy)-hexyl)-4-(4,4,5,5tetramethyl-1,3,2-dioxaborolan-2-yl)-piperidine-1-

carboxylate (4ah). This compound was synthesized according to general procedure A. The residue was purified by column chromatography on silica gel to afford the product 4ah (125.0 $\mathrm{mg}, 45 \%$ yield, $\mathrm{dr}>20: 1)$ as a white solid; ${ }^{1} \mathbf{H}$ NMR (400 MHz, Chloroform- $\left.\boldsymbol{d}\right) \delta 7.34-7.30$ (m, 4H), $7.30-7.26(\mathrm{~m}, 1 \mathrm{H}), 5.15-5.03(\mathrm{~m}, 2 \mathrm{H}), 4.59(\mathrm{dd}, J=7.8,2.6 \mathrm{~Hz}, 1 \mathrm{H}), 4.37(\mathrm{~d}, J=$ $11.7 \mathrm{~Hz}, 1 \mathrm{H}), 4.28(\mathrm{~d}, J=2.6 \mathrm{~Hz}, 1 \mathrm{H}), 4.22(\mathrm{dd}, J=8.0,1.7 \mathrm{~Hz}, 1 \mathrm{H}), 4.11(\mathrm{dtd}, J=11.5,7.2$, 6.6, $3.1 \mathrm{~Hz}, 1 \mathrm{H}), 4.01(\mathrm{~d}, J=11.7 \mathrm{~Hz}, 1 \mathrm{H}), 3.95-3.86(\mathrm{~m}, 2 \mathrm{H}), 3.75(\mathrm{~d}, J=13.0 \mathrm{~Hz}, 1 \mathrm{H}), 2.87$ $(\mathrm{td}, J=13.0,3.7 \mathrm{~Hz}, 1 \mathrm{H}), 2.28(\mathrm{t}, J=7.6 \mathrm{~Hz}, 2 \mathrm{H}), 1.81(\mathrm{dt}, J=13.6,5.9 \mathrm{~Hz}, 1 \mathrm{H}), 1.74-1.62$ (m, 3H), $1.61-1.53(\mathrm{~m}, 4 \mathrm{H}), 1.52(\mathrm{~s}, 3 \mathrm{H}), 1.46(\mathrm{~s}, 3 \mathrm{H}), 1.38(\mathrm{~s}, 3 \mathrm{H}), 1.32(\mathrm{~s}, 3 \mathrm{H}), 1.30-1.23$ (m, 5H), $1.22(\mathrm{~s}, 12 \mathrm{H}) \mathrm{ppm} ;{ }^{13} \mathbf{C}$ NMR (101 MHz, $\left.\mathbf{C D C l}_{3}\right) \delta$ 173.0, 155.8, 137.3, 128.5, 127.93, $127.87,109.2,108.8,101.7,83.4,70.9,70.6,70.2,66.9,65.2,61.3,52.1,38.3,34.1,31.9,29.2$, 26.6, 26.2, 26.0, 25.8, 25.4, 25.1, 24.9, 24.2 ppm; ${ }^{11}$ B NMR (128 MHz, Chloroform-d) $\delta$ 34.72 ppm; HRMS (ESI) calculated $[\mathrm{M}+\mathrm{H}]^{+}$for $\mathrm{C}_{37} \mathrm{H}_{56} \mathrm{BNO}_{11}{ }^{+}=702.4019$, found: 702.4018 .

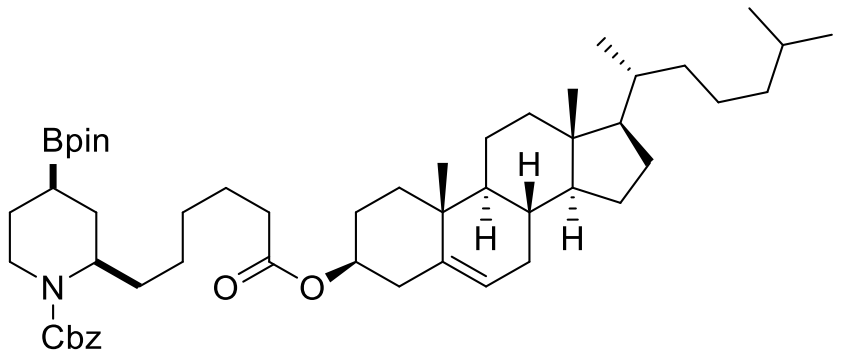

cis-benzyl 2-(6-(((3S,8S,9S,10R,13R, 14S,17R)-10,13-dimethyl-17-((R)6methylheptan-2-yl)-2,3,4,7,8,9,10, $11,12,13,14,15,16,17-t e t r a d e c a h y d$ ro-1H-cyclopenta[a]phenanthren-

3-yl)-oxy)-6-oxohexyl)-4-(4,4,5,5-tetramethyl-1,3,2-dioxaborolan-2-yl)-piperidine-1carboxylate (4ai). This compound was synthesized according to general procedure A. The residue was purified by column chromatography on silica gel to afford the product 4ai (231.0 mg, 70\% yield, $\mathrm{dr}>20: 1)$ as a white solid; ${ }^{1} \mathrm{H}$ NMR (400 MHz, Chloroform- $d$ ) $\delta 7.36-7.33$ (m, 4H), $7.32-7.27(\mathrm{~m}, 1 \mathrm{H}), 5.37(\mathrm{dd}, J=4.9,2.0 \mathrm{~Hz}, 1 \mathrm{H}), 5.17-5.07(\mathrm{~m}, 2 \mathrm{H}), 4.65-4.55$ (m, 1H), $4.20-4.08(\mathrm{~m}, 1 \mathrm{H}), 3.94(\mathrm{dt}, J=12.7,4.1 \mathrm{~Hz}, 1 \mathrm{H}), 2.89(\mathrm{td}, J=13.0,3.6 \mathrm{~Hz}, 1 \mathrm{H})$, $2.30(\mathrm{~d}, J=7.9 \mathrm{~Hz}, 2 \mathrm{H}), 2.22(\mathrm{t}, J=7.6 \mathrm{~Hz}, 2 \mathrm{H}), 2.04-1.92(\mathrm{~m}, 2 \mathrm{H}), 1.88-1.80(\mathrm{~m}, 4 \mathrm{H}), 1.79$ - $1.68(\mathrm{~m}, 2 \mathrm{H}), 1.68-1.56(\mathrm{~m}, 6 \mathrm{H}), 1.55-1.45(\mathrm{~m}, 6 \mathrm{H}), 1.44-1.28(\mathrm{~m}, 8 \mathrm{H}), 1.24(\mathrm{~s}, 12 \mathrm{H})$, 
$1.17-1.10(\mathrm{~m}, 5 \mathrm{H}), 1.07(\mathrm{~d}, \mathrm{~J}=9.2 \mathrm{~Hz}, 2 \mathrm{H}), 1.05-1.00(\mathrm{~m}, 5 \mathrm{H}), 0.99-0.94(\mathrm{~m}, 2 \mathrm{H}), 0.92$ (d, $\mathrm{J}=6.5 \mathrm{~Hz}, 3 \mathrm{H}), 0.87(\mathrm{~d}, \mathrm{~J}=1.9 \mathrm{~Hz}, 3 \mathrm{H}), 0.86(\mathrm{~d}, \mathrm{~J}=1.9 \mathrm{~Hz}, 3 \mathrm{H}), 0.68(\mathrm{~s}, 3 \mathrm{H}) \mathrm{ppm} ;{ }^{13} \mathbf{C} \mathbf{N M R}$ (101 MHz, $\left.\mathbf{C D C l}_{3}\right) \delta 173.3,155.8,139.8,137.3,128.5,127.9,127.9,122.7,83.4,73.8,66.8$, 56.8, 56.2, 52.0, 50.1, 42.4, 39.8, 39.6, 38.2, 37.1, 36.7, 36.3, 35.9, 34.7, 32.0, 31.9, 29.2, 28.3, $28.1,27.9,26.2,25.8,25.2,25.12,25.05,24.9,24.4,23.9,22.9,22.7,21.1,19.4,18.8,12.0$ ppm; ${ }^{11}$ B NMR (128 MHz, Chloroform-d) $\delta 35.65$ ppm; HRMS (ESI) calculated $[\mathrm{M}+\mathrm{H}]^{+}$for $\mathrm{C}_{52} \mathrm{H}_{83} \mathrm{BNO}_{6}{ }^{+}=828.6308$, found: 828.6308.

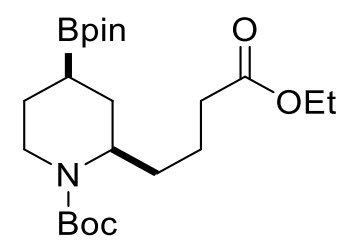

cis-tert-butyl-2-(4-ethoxy-4-oxobutyl)-4-(4,4,5,5-tetramethyl-1,3,2dioxaborolan-2-yl)-piperidine-1-carboxylate (4aj). This compound was synthesized according to general procedure A. The residue was purified by column chromatography on silica gel to afford the product 4aj (121.0 mg, 71\% yield, dr > 20:1) as a colorless oil; ${ }^{1} \mathbf{H}$ NMR (600 MHz, Chloroform- $\left.d\right) \delta$ $4.11(\mathrm{q}, J=7.2 \mathrm{~Hz}, 2 \mathrm{H}), 4.09-4.05(\mathrm{~m}, 1 \mathrm{H}), 3.90-3.85(\mathrm{~m}, 1 \mathrm{H}), 2.80(\mathrm{ddd}, J=13.7,12.3,3.8$ $\mathrm{Hz}, 1 \mathrm{H}), 2.36-2.23(\mathrm{~m}, 2 \mathrm{H}), 1.84(\mathrm{dt}, J=13.5,6.0 \mathrm{~Hz}, 1 \mathrm{H}), 1.74-1.69(\mathrm{~m}, 1 \mathrm{H}), 1.67-1.56$ (m, 5H), $1.45(\mathrm{~s}, 9 \mathrm{H}), 1.42$ - $1.37(\mathrm{~m}, 1 \mathrm{H}), 1.27$ - $1.22(\mathrm{~m}, 15 \mathrm{H}), 1.21-1.17(\mathrm{~m}, 1 \mathrm{H}) \mathrm{ppm} ;{ }^{13} \mathrm{C}$ NMR (151 MHz, $\left.\mathbf{C D C l}_{3}\right) \delta$ 173.6, 155.3, 83.3, 79.1, 60.3, 51.4, 37.7, 34.3, 31.7, 29.4, 28.6, 25.7, 25.0, 24.9, 22.0, 14.3 ppm; ${ }^{11}$ B NMR (128 MHz, Chloroform-d) $\delta 33.10$ ppm; HRMS (ESI) calculated $[\mathrm{M}+\mathrm{H}]^{+}$for $\mathrm{C}_{22} \mathrm{H}_{41} \mathrm{BNO}_{6}{ }^{+}=426.3021$, found: 426.3020 .

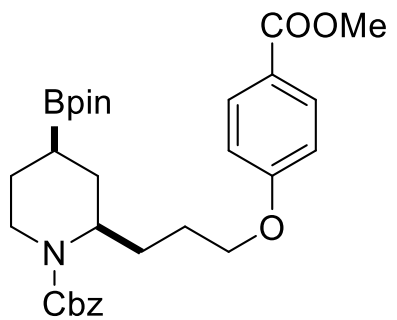

cis-benzyl-2-(3-(4-(methoxycarbonyl)-phenoxy)-propyl)-4(4,4,5,5-tetramethyl-1,3,2-dioxaborolan-2-yl)-piperidine-1carboxylate (4ak). This compound was synthesized according to general procedure A. The residue was purified by column chromatography on silica gel to afford the product 4ak $(136.0 \mathrm{mg}, 63 \%$ yield, $\mathrm{dr}>20: 1)$ as a colorless oil; ${ }^{1} \mathbf{H}$ NMR (400 MHz, Chloroform-d) $\delta 7.94(\mathrm{~d}, J=8.9 \mathrm{~Hz}, 2 \mathrm{H}), 7.36$ - $7.26(\mathrm{~m}$, 5H), 6.85 - 6.78 (m, 2H), 5.20 - $4.97(\mathrm{~m}, 2 \mathrm{H}), 4.21(\mathrm{dt}, J=12.6,4.9 \mathrm{~Hz}, 1 \mathrm{H}), 4.02$ - $3.91(\mathrm{~m}$, 3H), 3.87 (s, 3H), 2.99 - 2.84 (m, 1H), 1.92 - 1.77 (m, 3H), 1.74 - 1.67 (m, 3H), 1.64 - 1.53 (m, 2H), 1.26 - $1.21(\mathrm{~m}, 1 \mathrm{H}), 1.20(\mathrm{~d}, J=4.7 \mathrm{~Hz}, 12 \mathrm{H}) \mathrm{ppm} ;{ }^{13} \mathbf{C} \mathbf{N M R}\left(\mathbf{1 0 1} \mathbf{M H z}, \mathbf{C D C l}_{3}\right) \delta$ 167.0, 
$162.9,155.9,137.1,131.7,128.6,128.0,127.9,122.5,114.1,83.5,67.9,67.0,52.0,51.8,38.2$, 29.6, 28.6, 26.2, 25.8, 25.0, 24.9 ppm; ${ }^{11}$ B NMR (128 MHz, Chloroform-d) $\delta 34.30$ ppm; HRMS (ESI) calculated $[\mathrm{M}+\mathrm{H}]^{+}$for $\mathrm{C}_{30} \mathrm{H}_{41} \mathrm{BNO}_{7}{ }^{+}=538.2971$, found: 538.2970.

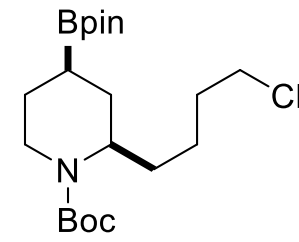

cis-tert-butyl-2-(4-chlorobutyl)-4-(4,4,5,5-tetramethyl-1,3,2dioxaborolan-2-yl)-piperidine-1-carboxylate (4al). This compound was synthesized according to general procedure A. The residue was purified by column chromatography on silica gel to afford the product 4 al (81.0 mg, 50\% yield, $\mathrm{dr}>20: 1)$ as a colorless oil; ${ }^{\mathbf{1}} \mathbf{H}$ NMR (600 MHz, Chloroform- $d$ ) $\delta 4.06$ (td, $J=9.7,8.1,4.4 \mathrm{~Hz}, 1 \mathrm{H}), 3.87(\mathrm{dt}, J=13.7,3.5 \mathrm{~Hz}, 1 \mathrm{H}), 3.52(\mathrm{t}, J=6.8 \mathrm{~Hz}, 2 \mathrm{H}), 2.80$ (ddd, $J=13.6,12.3,3.7 \mathrm{~Hz}, 1 \mathrm{H}), 1.85-1.70(\mathrm{~m}, 4 \mathrm{H}), 1.67-1.57(\mathrm{~m}, 3 \mathrm{H}), 1.50-1.46(\mathrm{~m}, 1 \mathrm{H}), 1.45$ (s, 9H), $1.42-1.35(\mathrm{~m}, 2 \mathrm{H}), 1.25(\mathrm{~s}, 12 \mathrm{H}), 1.21-1.17(\mathrm{~m}, 1 \mathrm{H}) \mathrm{ppm} ;{ }^{13} \mathbf{C}$ NMR (101 MHz, $\left.\mathbf{C D C l}_{3}\right) \delta 155.4,83.4,79.1,51.6,45.1,37.9,32.6,31.5,29.1,28.6,25.8,25.1,24.9,24.0$ ppm; ${ }^{11}$ B NMR (128 MHz, Chloroform-d) $\delta 33.17$ ppm; HRMS (ESI) calculated $[\mathrm{M}+\mathrm{H}]^{+}$for $\mathrm{C}_{20} \mathrm{H}_{38} \mathrm{BClNO}_{4}{ }^{+}=402.2577$, found: 402.2573 .<smiles>O=C(c1ccccc1)N1CCC(Br)CC1C1CCCCC1</smiles>
cis-benzyl-2-cyclohexyl-4-(4,4,5,5-tetramethyl-1,3,2-dioxaborolan-2-yl)piperidine-1-carboxylate (4am). This compound was synthesized according to general procedure A using bromocyclohexane ( $0.8 \mathrm{mmol}, 2.0$ equiv) and L7 instead of L10. The residue was purified by column chromatography on silica gel to afford the product $\mathbf{4 a m}(131.0 \mathrm{mg}, 77 \%$ yield, $\mathrm{dr}=8: 1)$ as a colorless oil; ${ }^{\mathbf{1}} \mathbf{H}$ NMR (600 MHz, Chloroform-d) $\delta 7.36-7.34(\mathrm{~m}, 4 \mathrm{H}), 7.31-7.28(\mathrm{~m}, 1 \mathrm{H}), 5.12(\mathrm{~s}, 2 \mathrm{H}), 3.98(\mathrm{~d}, \mathrm{~J}$ $=13.2 \mathrm{~Hz}, 1 \mathrm{H}), 3.87-3.77(\mathrm{~m}, 1 \mathrm{H}), 2.74(\mathrm{td}, \mathrm{J}=13.7,12.8,3.8 \mathrm{~Hz}, 1 \mathrm{H}), 1.90(\mathrm{dt}, \mathrm{J}=12.8,3.1$ $\mathrm{Hz}, 1 \mathrm{H}), 1.85(\mathrm{dt}, \mathrm{J}=13.8,4.7 \mathrm{~Hz}, 1 \mathrm{H}), 1.76-1.68(\mathrm{~m}, 4 \mathrm{H}), 1.66-1.60(\mathrm{~m}, 2 \mathrm{H}), 1.60-1.50$ $(\mathrm{m}, 2 \mathrm{H}), 1.26(\mathrm{~d}, \mathrm{~J}=7.1 \mathrm{~Hz}, 12 \mathrm{H}), 1.21-1.15(\mathrm{~m}, 2 \mathrm{H}), 1.14-1.07(\mathrm{~m}, 2 \mathrm{H}), 0.98-0.84(\mathrm{~m}$, 2H) ppm; ${ }^{13}$ C NMR (151 MHz, CDCl3) $\delta$ 156.2, 137.4, 128.5, 127.8, 127.7, 83.6, 83.3, 66.8, 57.48, 38.9, 37.7, 30.1, 29.3, 26.4, 26.3, 26.1, 26.0, 25.7, 25.4, 25.1, 24.6 ppm; ${ }^{11}$ B NMR (128 MHz, Chloroform-d) $\delta 33.4,30.0$ ppm; HRMS (ESI) calculated $[\mathrm{M}+\mathrm{H}]^{+}$for $\mathrm{C}_{25} \mathrm{H}_{39} \mathrm{BNO}_{4}{ }^{+}=$ 428.2967, found: 428.2973 . 


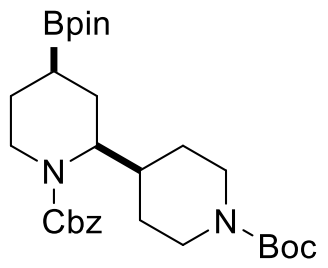

cis-1-benzyl-1' -(tert-butyl)-4-(4,4,5,5-tetramethyl-1,3,2 dioxaborolan-2-yl)-[2,4'-bipiperidine]-1,1'-dicarboxylate (4an). This compound was synthesized according to general procedure A using 1-N-Boc-4-bromopiperidine ( $0.8 \mathrm{mmol}, 2.0$ equiv) and L7 instead of L10. The residue was purified by column chromatography on silica gel to afford the product 4an (86.8 mg, 41\% yield, $\mathrm{dr}>20: 1)$ as a colorless oil; ${ }^{1} \mathbf{H}$ NMR (600 MHz, Chloroform-d) $\delta$ 7.37 - $7.32(\mathrm{~m}, 4 \mathrm{H}), 7.32$ - $7.29(\mathrm{~m}, 1 \mathrm{H}), 5.12(\mathrm{~s}, 2 \mathrm{H}), 4.20$ - $3.95(\mathrm{~m}, 3 \mathrm{H}), 3.91$ - $3.78(\mathrm{~m}, 1 \mathrm{H})$, $2.77-2.70(\mathrm{~m}, 1 \mathrm{H}), 2.67-2.49(\mathrm{~m}, 2 \mathrm{H}), 1.89-1.79(\mathrm{~m}, 2 \mathrm{H}), 1.78-1.69(\mathrm{~m}, 3 \mathrm{H}), 1.65-1.61$ (m, 1H), $1.52-1.47(\mathrm{~m}, 1 \mathrm{H}), 1.45(\mathrm{~s}, 9 \mathrm{H}), 1.25(\mathrm{~d}, \mathrm{~J}=6.7 \mathrm{~Hz}, 12 \mathrm{H}), 1.22-1.18(\mathrm{~m}, 1 \mathrm{H}), 1.17$ - 1.06 (m, 2H) ppm; ${ }^{13} \mathbf{C}$ NMR (151 MHz, CDCl3) $\delta 156.1,154.9,137.1,128.6,128.0,127.9$, 83.4, 79.4, 67.0, 56.9, 43.5, 39.0, 36.4, 28.9, 25.7, 25.6, 25.4, 25.0, 24.6 ppm; ${ }^{11}$ B NMR (128 MHz, Chloroform-d) $\delta 34.5$ ppm; HRMS (ESI) calculated $[\mathrm{M}+\mathrm{H}]^{+}$for $\mathrm{C}_{29} \mathrm{H}_{46} \mathrm{BN}_{2} \mathrm{O}_{6}{ }^{+}=$ 529.3443, found: 529.3452 .

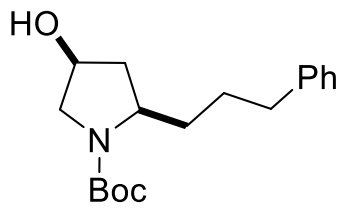

cis-tert-butyl 4-hydroxy-2-(3-phenylpropyl)-pyrrolidine-1carboxylate (4ao). This compound was synthesized according to general procedure A. The residue was purified by column chromatography on silica gel to afford the product 4 ao $(72.0 \mathrm{mg}, 59 \%$ yield, $\mathrm{dr}>20: 1)$ as a white solid; ${ }^{1} \mathbf{H}$ NMR (600 MHz, Chloroform-d) $\delta 7.30$ - 7.24 (m, 2H), 7.20 - 7.14 (m, 3H), $4.41-4.35(\mathrm{~m}, 1 \mathrm{H}), 3.94-3.56(\mathrm{~m}, 2 \mathrm{H}), 3.24(\mathrm{ddd}, J=11.9,3.6,1.1 \mathrm{~Hz}, 1 \mathrm{H}), 2.74-2.46(\mathrm{~m}$, 2H), $2.16(\mathrm{ddd}, J=13.9,8.4,5.9 \mathrm{~Hz}, 1 \mathrm{H}), 2.10-1.82(\mathrm{~m}, 2 \mathrm{H}), 1.76-1.71(\mathrm{~m}, 1 \mathrm{H}), 1.67-1.56$ (m, 3H), 1.43 (s, 9H) ppm; ${ }^{13}$ C NMR (151 MHz, Chloroform-d) $\delta$ 154.7, 142.6, 128.5, 128.4, 125.8, 79.5, 71.0, 70.2 57.0, 54.7, 39.1, 36.0, 35.0, 28.6 ppm; HRMS (ESI) calculated [M+H] $]^{+}$ for $\mathrm{C}_{18} \mathrm{H}_{28} \mathrm{NO}_{3}{ }^{+}=306.2064$, found: 306.2057<smiles>CC(=O)N1CCC(Br)CC1[N+](=O)[O-]</smiles>

cis-benzyl-2-methyl-4-(4,4,5,5-tetramethyl-1,3,2-dioxaborolan-2-yl)piperidine-1-carboxylate (4ap). This compound was synthesized according to general procedure A using $\mathrm{CH}_{3} \mathrm{I}(0.8 \mathrm{mmol}, 2.0$ equiv $), \mathrm{L} 8$, stirring at $30{ }^{\circ} \mathrm{C}$. The 
residue was purified by column chromatography on silica gel to afford the product 4ap (50.4 mg, 35\% yield, $\mathrm{dr}=8: 1)$ as a colorless oil; ${ }^{1} \mathbf{H}$ NMR (400 MHz, Chloroform-d) $\delta 7.35-7.32$ (m, 4H), 7.32 - $7.27(\mathrm{~m}, 1 \mathrm{H}), 5.11(\mathrm{~s}, 2 \mathrm{H}), 4.26$ (pd, J = 6.6, $4.4 \mathrm{~Hz}, 1 \mathrm{H}), 3.89$ (ddd, J = 13.6, 5.4, $2.7 \mathrm{~Hz}, 1 \mathrm{H}), 2.99$ (ddd, J = 13.6, 12.2, 4.0 Hz, 1H), 1.85 (dt, J = 13.6, $5.9 \mathrm{~Hz}, 1 \mathrm{H}), 1.74$ $(\mathrm{dq}, \mathrm{J}=12.3,3.8 \mathrm{~Hz}, 1 \mathrm{H}), 1.66-1.63(\mathrm{~m}, 1 \mathrm{H}), 1.61-1.56(\mathrm{~m}, 1 \mathrm{H}), 1.24(\mathrm{~s}, 12 \mathrm{H}), 1.21-1.17$ $(\mathrm{m}, 1 \mathrm{H}), 1.13(\mathrm{~d}, \mathrm{~J}=6.9 \mathrm{~Hz}, 3 \mathrm{H}) \mathrm{ppm} ;{ }^{13} \mathbf{C}$ NMR (101 MHz, CDCl3) $\delta$ 155.7, 137.4, 128.6, 127.9, 127.8, 83.4, 66.8, 48.1, 37.9, 31.1, 25.6, 25.00, 24.96, 18.2 ppm; ${ }^{11}$ B NMR (128 MHz, Chloroform-d) $\delta 34.7 \mathrm{ppm}$; HRMS (ESI) calculated $[\mathrm{M}+\mathrm{H}]^{+}$for $\mathrm{C}_{20} \mathrm{H}_{31} \mathrm{BNO}_{4}^{+}=360.2341$, found: 360.2346 .<smiles>O=C(c1ccccc1)N1C(CCCc2ccccc2)CC(Br)CC1c1ccccc1</smiles>

cis-benzyl-2-phenyl-6-(3-phenylpropyl)-4-(4,4,5,5-tetramethyl1,3,2-dioxaborolan-2-yl)-piperidine-1-carboxylate (4aq). This compound was synthesized according to general procedure A. The residue was purified by column chromatography on silica gel to afford the product 4aq (99.6 mg, 46\% yield, $\mathrm{dr}>20: 1)$ as a colorless oil; ${ }^{1} \mathbf{H}$ NMR (400 MHz, Chloroform-d $) \delta 7.34-7.21(\mathrm{~m}, 10 \mathrm{H}), 7.20-7.12(\mathrm{~m}, 5 \mathrm{H}), 5.24(\mathrm{t}, J=3.9 \mathrm{~Hz}, 1 \mathrm{H}), 5.16-$ $4.98(\mathrm{~m}, 2 \mathrm{H}), 3.96(\mathrm{t}, J=6.3 \mathrm{~Hz}, 1 \mathrm{H}), 2.58(\mathrm{t}, J=7.5 \mathrm{~Hz}, 2 \mathrm{H}), 2.29-2.11(\mathrm{~m}, 2 \mathrm{H}), 1.93-1.83$ $(\mathrm{m}, 2 \mathrm{H}), 1.83-1.65(\mathrm{~m}, 3 \mathrm{H}), 1.62-1.53(\mathrm{~m}, 1 \mathrm{H}), 1.22(\mathrm{~d}, J=2.1 \mathrm{~Hz}, 12 \mathrm{H}), 1.15$ - $1.04(\mathrm{~m}$, 1H) ppm; ${ }^{13} \mathbf{C}$ NMR (101 MHz, $\left.\mathbf{C D C l}_{3}\right) \delta$ 156.4, 142.7, 137.0, 128.5, 128.41, 128.38, 128.3, $127.8,126.4,126.0,125.8,83.4,66.9,55.5,52.8,36.3,36.1,29.1,28.4,25.1,24.9,24.8$ ppm; ${ }^{11}$ B NMR (128 MHz, Chloroform-d) $\delta 35.43$ ppm; HRMS (ESI) calculated $[\mathrm{M}+\mathrm{H}]^{+}$for $\mathrm{C}_{34} \mathrm{H}_{43} \mathrm{BNO}_{4}^{+}=540.3280$, found: 540.3268 .<smiles>CCOC(=O)CCCC1CC(Br)CC(c2ccccc2)N1C(=O)OCc1ccccc1</smiles>

cis-benzyl-2-(4-ethoxy-4-oxobutyl)-6-phenyl-4-(4,4,5,5tetramethyl-1,3,2-dioxaborolan-2-yl)-piperidine-1-carboxylate (4ar). This compound was synthesized according to general procedure A on a $0.2 \mathrm{mmol}$ scale. The residue was purified by column chromatography on silica gel to afford the product $4 \mathbf{a r}(60.9 \mathrm{mg}, 57 \%$ yield, $\mathrm{dr}>20: 1)$ as a colorless oil; ${ }^{1} \mathbf{H}$ NMR (400 MHz, Chloroform-d) $\delta 7.37$ - 7.07 (m, 10H), $5.23(\mathrm{t}, J=3.7$ 
$\mathrm{Hz}, 1 \mathrm{H}), 5.12-4.99(\mathrm{~m}, 2 \mathrm{H}), 4.10(\mathrm{q}, J=7.1 \mathrm{~Hz}, 2 \mathrm{H}), 3.90(\mathrm{dt}, J=8.7,5.0 \mathrm{~Hz}, 1 \mathrm{H}), 2.31-$ $2.09(\mathrm{~m}, 4 \mathrm{H}), 1.91-1.75(\mathrm{~m}, 4 \mathrm{H}), 1.67-1.50(\mathrm{~m}, 2 \mathrm{H}), 1.24(\mathrm{t}, J=7.2 \mathrm{~Hz}, 3 \mathrm{H}), 1.20(\mathrm{~d}, J=2.0$ $\mathrm{Hz}, 12 \mathrm{H}), 1.16$ - $1.08(\mathrm{~m}, 1 \mathrm{H}) \mathrm{ppm} ;{ }^{13} \mathbf{C}$ NMR (101 MHz, $\left.\mathbf{C D C l}_{3}\right) \delta$ 173.7, 156.3, 136.9, 128.4, $128.3,127.8,126.4,125.9,83.4,66.9,60.3,55.6,52.6,35.7,34.4,28.4,25.2,24.9,24.8,22.5$, 14.4 ppm; ${ }^{11}$ B NMR (128 MHz, Chloroform-d) $\delta 35.80$ ppm; HRMS (ESI) calculated [M+H] ${ }^{+}$ for $\mathrm{C}_{31} \mathrm{H}_{43} \mathrm{BNO}_{6}{ }^{+}=536.31780$, found: 536.3168 .

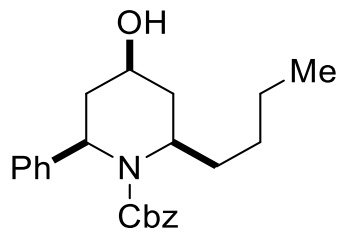

cis-benzyl-2-butyl-4-hydroxy-6-phenylpiperidine-1-carboxylate (4as). This compound was synthesized according to general procedure A on a $0.2 \mathrm{mmol}$ scale. The residue was purified by column chromatography on silica gel to afford the product 4as $(34.7 \mathrm{mg}, 47 \%$ yield, $\mathrm{dr}>20: 1)$ as a colorless oil; ${ }^{1} \mathbf{H}$ NMR (600 MHz, Chloroform-d $) \delta 7.35$ - $7.13(\mathrm{~m}, 10 \mathrm{H})$, $5.42(\mathrm{t}, J=4.2 \mathrm{~Hz}, 1 \mathrm{H}), 5.14-5.07$ (m, 2H), 3.89 (dq, $J=11.2,5.9 \mathrm{~Hz}, 1 \mathrm{H}), 3.66$ (dq, $J=10.6$, $5.4 \mathrm{~Hz}, 1 \mathrm{H}), 2.62(\mathrm{ddd}, J=13.9,6.4,3.2 \mathrm{~Hz}, 1 \mathrm{H}), 2.12-1.95(\mathrm{~m}, 3 \mathrm{H}), 1.74-1.65(\mathrm{~m}, 3 \mathrm{H})$, $1.34-1.22(\mathrm{~m}, 4 \mathrm{H}), 0.89-0.85(\mathrm{~m}, 3 \mathrm{H}) \mathrm{ppm} ;{ }^{13} \mathbf{C} \mathbf{N M R}\left(\mathbf{1 0 1} \mathbf{~ M H z}, \mathbf{C D C l}_{3}\right) \delta$ 156.4, 141.5, $136.7,128.6,128.5,127.99,127.95,126.7,125.9,67.1,63.8,54.7,52.8,36.4,35.9,35.5,29.3$, 22.7, 14.2 ppm; HRMS (ESI) calculated $[\mathrm{M}+\mathrm{H}]^{+}$for $\mathrm{C}_{23} \mathrm{H}_{30} \mathrm{NO}_{3}{ }^{+}=368.2220$, found: 368.2221.

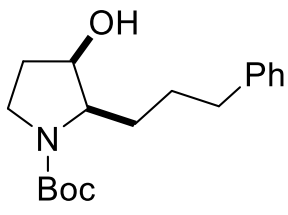

cis-tert-butyl 3-hydroxy-2-(3-phenylpropyl)-pyrrolidine-1carboxylate (4at). This compound was synthesized according to general procedure A. The residue was purified by column chromatography on silica gel to afford the product 4at $(49.8 \mathrm{mg}, 41 \%$ yield, $\mathrm{dr}>20: 1)$ as a colorless oil; ${ }^{1} \mathbf{H}$ NMR (400 MHz, Chloroform-d) $\delta 7.30-7.27(\mathrm{~m}, 1 \mathrm{H}), 7.26-7.25(\mathrm{~m}, 1 \mathrm{H}), 7.20-7.14(\mathrm{~m}, 3 \mathrm{H})$, $4.41-4.28(\mathrm{~m}, 1 \mathrm{H}), 3.74(\mathrm{~s}, 1 \mathrm{H}), 3.48-3.41(\mathrm{~m}, 1 \mathrm{H}), 3.35$ (ddd, $J=11.1,7.9,6.1 \mathrm{~Hz}, 1 \mathrm{H})$, $2.65(\mathrm{t}, J=6.6 \mathrm{~Hz}, 2 \mathrm{H}), 2.00(\mathrm{dq}, J=13.4,6.5 \mathrm{~Hz}, 1 \mathrm{H}), 1.82(\mathrm{ddd}, J=14.6,12.9,7.0 \mathrm{~Hz}, 1 \mathrm{H})$, $1.76-1.69(\mathrm{~m}, 3 \mathrm{H}), 1.68-1.61(\mathrm{~m}, 2 \mathrm{H}), 1.42(\mathrm{~s}, 9 \mathrm{H}) \mathrm{ppm} ;{ }^{13} \mathbf{C} \mathbf{~ N M R}\left(\mathbf{1 5 1} \mathbf{~ M H z}, \mathbf{C D C l}_{3}\right) \delta$ 155.1, 142.6, 128.5, 128.4, 125.8, 79.5, 72.0, 60.3, 43.3, 36.3, 31.8, 28.6, 28.6 ppm; HRMS (ESI) calculated $[\mathrm{M}+\mathrm{H}]^{+}$for $\mathrm{C}_{18} \mathrm{H}_{28} \mathrm{NO}_{3}{ }^{+}=306.2064$, found: 306.2063 . 


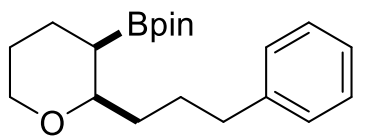

cis-4,4,5,5-tetramethyl-2-(2-(3-phenylpropyl)-tetrahydro-2Hpyran-3-yl)-1,3,2-dioxaborolane (4au). This compound was synthesized according to general procedure A. The residue was purified by column chromatography on silica gel to afford the product $4 \mathbf{a u}(46.5 \mathrm{mg}, 35 \%$ yield, $\mathrm{dr}>20: 1)$ as a colorless oil; ${ }^{1}$ H NMR (600 MHz, Chloroform-d) $\delta 7.27$ - 7.23 (m, 2H), 7.19 - 7.13 (m, 3H), $3.98-3.93(\mathrm{~m}, 1 \mathrm{H}), 3.45-3.33(\mathrm{~m}, 2 \mathrm{H}), 2.65-2.58(\mathrm{~m}, 2 \mathrm{H}), 1.92-1.85(\mathrm{~m}, 1 \mathrm{H}), 1.81-1.68$ $(\mathrm{m}, 3 \mathrm{H}), 1.67-1.63(\mathrm{~m}, 1 \mathrm{H}), 1.62-1.57(\mathrm{~m}, 1 \mathrm{H}), 1.50-1.44(\mathrm{~m}, 1 \mathrm{H}), 1.41-1.37(\mathrm{~m}, 1 \mathrm{H})$, $1.27-1.24(\mathrm{~m}, 1 \mathrm{H}), 1.22(\mathrm{~d}, J=3.7 \mathrm{~Hz}, 12 \mathrm{H}) \mathrm{ppm} ;{ }^{13} \mathbf{C}$ NMR (151 MHz, Chloroform-d $) \delta$ $142.9,128.6,128.3,125.7,83.0,79.5,68.3,36.1,34.6,28.2,25.7,25.1,24.9,24.8$ ppm; ${ }^{11} \mathbf{B}$ NMR (128 MHz, Chloroform-d) $\delta 32.09$ ppm; HRMS (ESI) calculated $[\mathrm{M}+\mathrm{H}]^{+}$for $\mathrm{C}_{20} \mathrm{H}_{32} \mathrm{BO}_{3}{ }^{+}=331.2439$, found: 331.2440 .

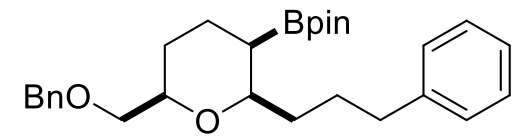

cis-2-(6-((benzyloxy)methyl)-2-(3-phenylpropyl)tetrahydro-2H-pyran-3-yl)-4,4,5,5-tetramethyl-1,3,2dioxaborolane (4av). This compound was synthesized according to general procedure A. The residue was purified by column chromatography on silica gel to afford the product $\mathbf{4 a v}$ (35.0 mg, 19\% yield, $\mathrm{dr}>20: 1)$ as a colorless oil; ${ }^{1} \mathbf{H}$ NMR (600 MHz, Chloroform- $\left.\boldsymbol{d}\right) \delta 7.36-7.28$ (m, 4H), 7.28 - $7.24(\mathrm{~m}, 1 \mathrm{H}), 7.26-7.22(\mathrm{~m}, 2 \mathrm{H}), 7.20$ - $7.12(\mathrm{~m}, 3 \mathrm{H}), 4.60$ - $4.56(\mathrm{~m}, 1 \mathrm{H})$, $4.55-4.49(\mathrm{~m}, 1 \mathrm{H}), 3.57-3.52(\mathrm{~m}, 2 \mathrm{H}), 3.43-3.36(\mathrm{~m}, 2 \mathrm{H}), 2.68-2.55(\mathrm{~m}, 2 \mathrm{H}), 2.00-1.94$ (m, 1H), $1.84-1.72(\mathrm{~m}, 2 \mathrm{H}), 1.68-1.65(\mathrm{~m}, 1 \mathrm{H}), 1.64-1.57(\mathrm{~m}, 2 \mathrm{H}), 1.53-1.43(\mathrm{~m}, 2 \mathrm{H})$, 1.28 - $1.24(\mathrm{~m}, 1 \mathrm{H}), 1.20(\mathrm{~d}, J=3.2 \mathrm{~Hz}, 12 \mathrm{H}) \mathrm{ppm} ;{ }^{13} \mathbf{C} \mathbf{N M R}\left(\mathbf{1 5 1} \mathbf{~ M H z}, \mathbf{C D C l}_{3}\right) \delta$ 143.0, $138.8,128.6,128.4,128.3,127.8,127.5,125.6,82.9,80.4,77.8,74.0,73.3,36.2,35.2,28.4$, 27.1, 26.2, 24.9, 24.8 ppm; ${ }^{11}$ B NMR (128 MHz, Chloroform-d) $\delta 32.75$ ppm; HRMS (ESI) calculated $[\mathrm{M}+\mathrm{H}]^{+}$for $\mathrm{C}_{28} \mathrm{H}_{40} \mathrm{BO}_{4}{ }^{+}=451.3014$, found: 451.3018 .

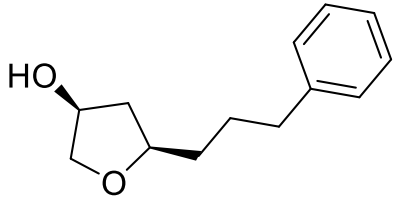

(3S,5R)-5-(3-phenylpropyl)-tetrahydrofuran-3-ol (4a). This compound was synthesized according to general procedure B. The residue was purified by column chromatography on silica gel to 
afford the product $4 \mathbf{4}(55.0 \mathrm{mg}, 67 \%$ yield, $\mathrm{dr}>20: 1)$ as a colorless oil; ${ }^{1} \mathbf{H}$ NMR (600 MHz, Chloroform-d) $\delta 7.32-7.24(\mathrm{~m}, 2 \mathrm{H}), 7.20-7.15(\mathrm{~m}, 3 \mathrm{H}), 4.46-4.41(\mathrm{~m}, 1 \mathrm{H}), 3.89$ - $3.74(\mathrm{~m}$, 2H), 3.66 (dd, $J=9.9,4.4 \mathrm{~Hz}, 1 \mathrm{H}), 2.65$ (ddd, $J=8.2,6.6,2.8 \mathrm{~Hz}, 2 \mathrm{H}), 2.32$ (ddd, $J=13.4$, 7.6, $6.8 \mathrm{~Hz}, 1 \mathrm{H}), 1.84-1.71(\mathrm{~m}, 3 \mathrm{H}), 1.70-1.66(\mathrm{~m}, 1 \mathrm{H}), 1.64-1.58(\mathrm{~m}, 1 \mathrm{H}), 1.49$ (dddd, $J=$ 13.5, 7.3, 3.0, $1.4 \mathrm{~Hz}, 1 \mathrm{H}) \mathrm{ppm} ;{ }^{13} \mathbf{C}$ NMR (151 MHz, Chloroform-d) $\delta 142.4,128.5,128.4$, 125.9, 79.2, 75.5, 72.6, 41.5, 35.9, 35.8, 28.2 ppm; HRMS (ESI) calculated $[\mathrm{M}+\mathrm{H}]^{+}$for $\mathrm{C}_{13} \mathrm{H}_{19} \mathrm{O}_{2}{ }^{+}=207.1380$, found: $207.1376 .[\boldsymbol{\alpha}]^{\mathbf{2 0}} \mathbf{D}=-5.8\left(\mathrm{c}=0.88, \mathrm{CHCl}_{3}\right)$. HPLC analysis (ODH, $n$-Hexane: $i$-Propanol $=95: 5$ as eluent, $1 \mathrm{~mL} / \mathrm{min}, 220 \mathrm{~nm})$ indicated $94 \%$ ee: $\mathrm{t}_{\mathrm{R}}($ minor $)=$ $11.9 \mathrm{~min}, \mathrm{t}_{\mathrm{R}}($ major $)=13.7 \mathrm{~min}$.
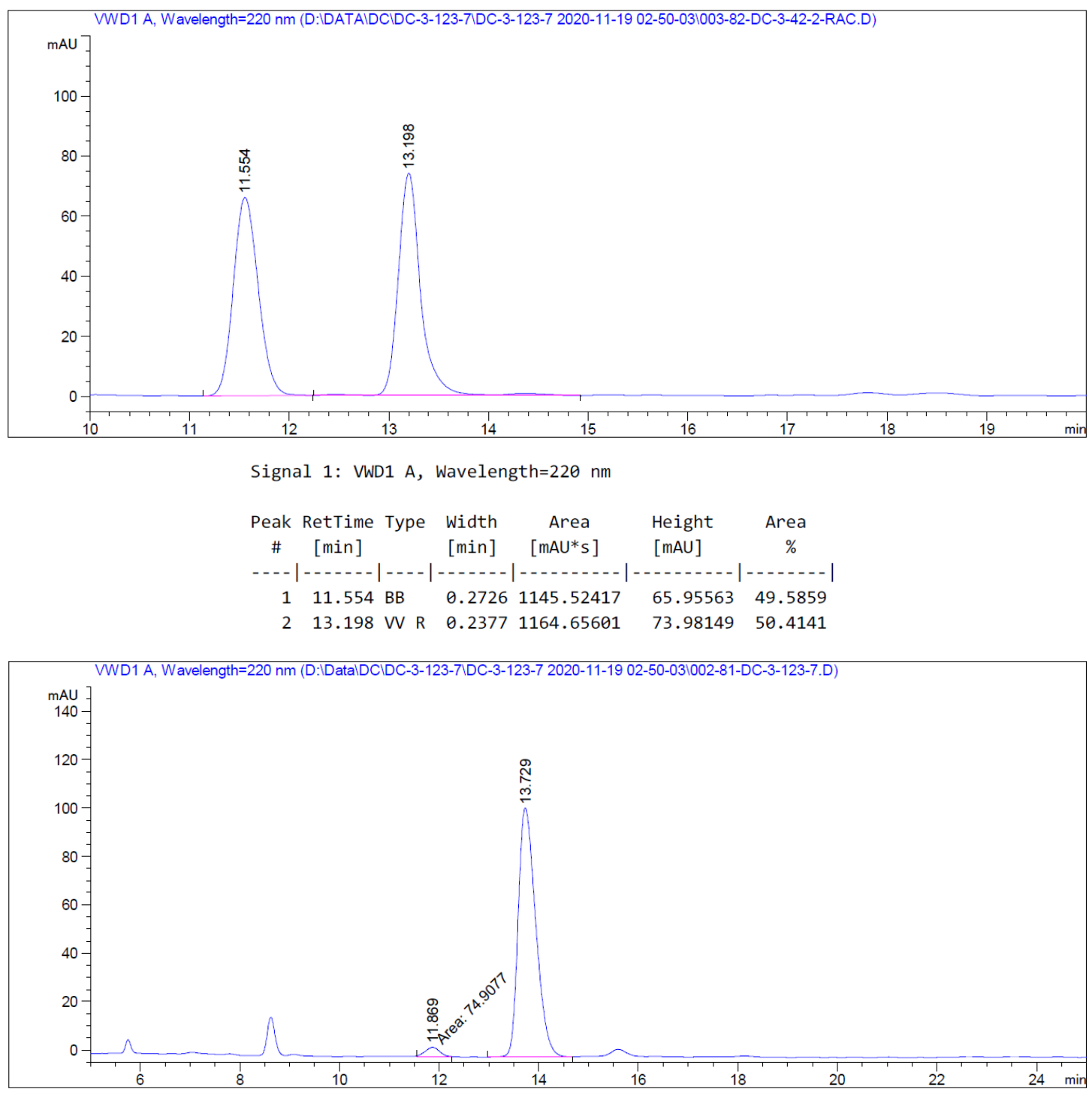

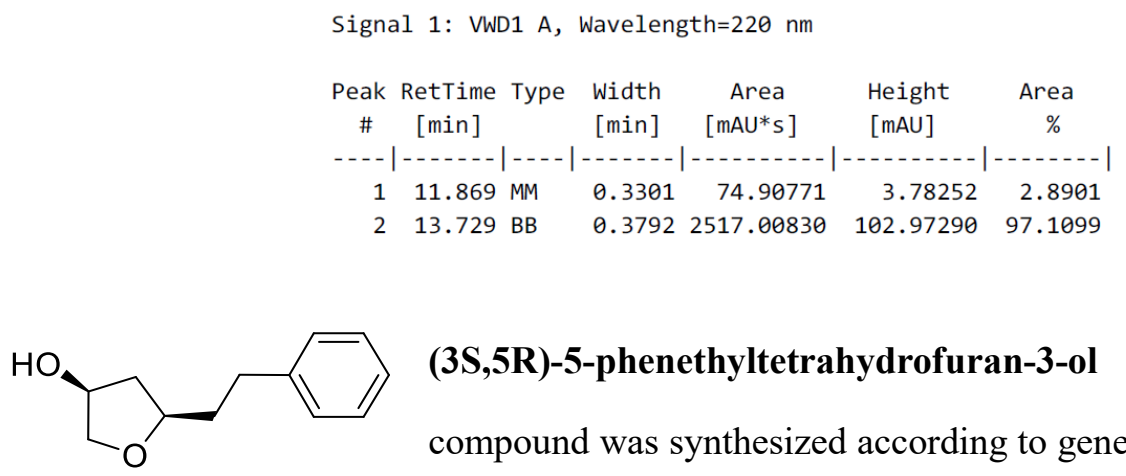

(3S,5R)-5-phenethyltetrahydrofuran-3-ol (4aw). This compound was synthesized according to general procedure B. The residue was purified by column chromatography on silica gel to afford the product 4aw (48.0 mg, 62\% yield, $\mathrm{dr}>20: 1)$ as a colorless oil; ${ }^{1} \mathbf{H}$ NMR (600 MHz, Chloroform- $d$ ) $\delta 7.30-7.26$ (m, 2H), $7.22-7.16(\mathrm{~m}, 3 \mathrm{H}), 4.45(\mathrm{~s}, 1 \mathrm{H}), 3.86(\mathrm{dt}, J=9.9,1.6 \mathrm{~Hz}, 1 \mathrm{H}), 3.81(\mathrm{qd}, J=7.5,5.3$ Hz, 1H), 3.69 (dd, $J=9.9,4.4 \mathrm{~Hz}, 1 \mathrm{H}), 2.79$ (ddd, $J=13.7,9.9,5.6 \mathrm{~Hz}, 1 \mathrm{H}), 2.68$ (ddd, $J=$ 13.8, 9.7, 6.5 Hz, 1H), 2.34 (ddd, $J=13.5,7.6,6.8 \mathrm{~Hz}, 1 \mathrm{H}), 2.04$ (dddd, $J=13.4,9.8,7.7,5.7$ $\mathrm{Hz}, 1 \mathrm{H}), 1.89$ (dddd, $J=13.1,9.5,6.2,5.1 \mathrm{~Hz}, 2 \mathrm{H}), 1.53$ (dddd, $J=13.5,7.2,2.9,1.3 \mathrm{~Hz}, 1 \mathrm{H}$ ) ppm; ${ }^{13} \mathbf{C}$ NMR (151 MHz, $\left.\mathbf{C D C l}_{3}\right) \delta 141.9,128.6,128.5,126.0,78.5,75.6,72.7,41.5,37.9$, 32.7 ppm; HRMS (ESI) calculated $[\mathrm{M}+\mathrm{H}]^{+}$for $\mathrm{C}_{12} \mathrm{H}_{17} \mathrm{O}_{2}{ }^{+}=193.1223$, found: 193.1222 . [ $[\boldsymbol{\alpha}]^{\mathbf{2 0}} \mathbf{D}$ $=+4.8\left(\mathrm{c}=0.23, \mathrm{CHCl}_{3}\right)$. HPLC analysis $(\mathrm{OD}-\mathrm{H}, n$-Hexane: $i$-Propanol $=95: 5$ as eluent, 1 $\mathrm{mL} / \min , 220 \mathrm{~nm}$ ) indicated 96\% ee: $\mathrm{t}_{\mathrm{R}}($ minor $)=13.7 \mathrm{~min}, \mathrm{t}_{\mathrm{R}}($ major $)=21.4 \mathrm{~min}$.

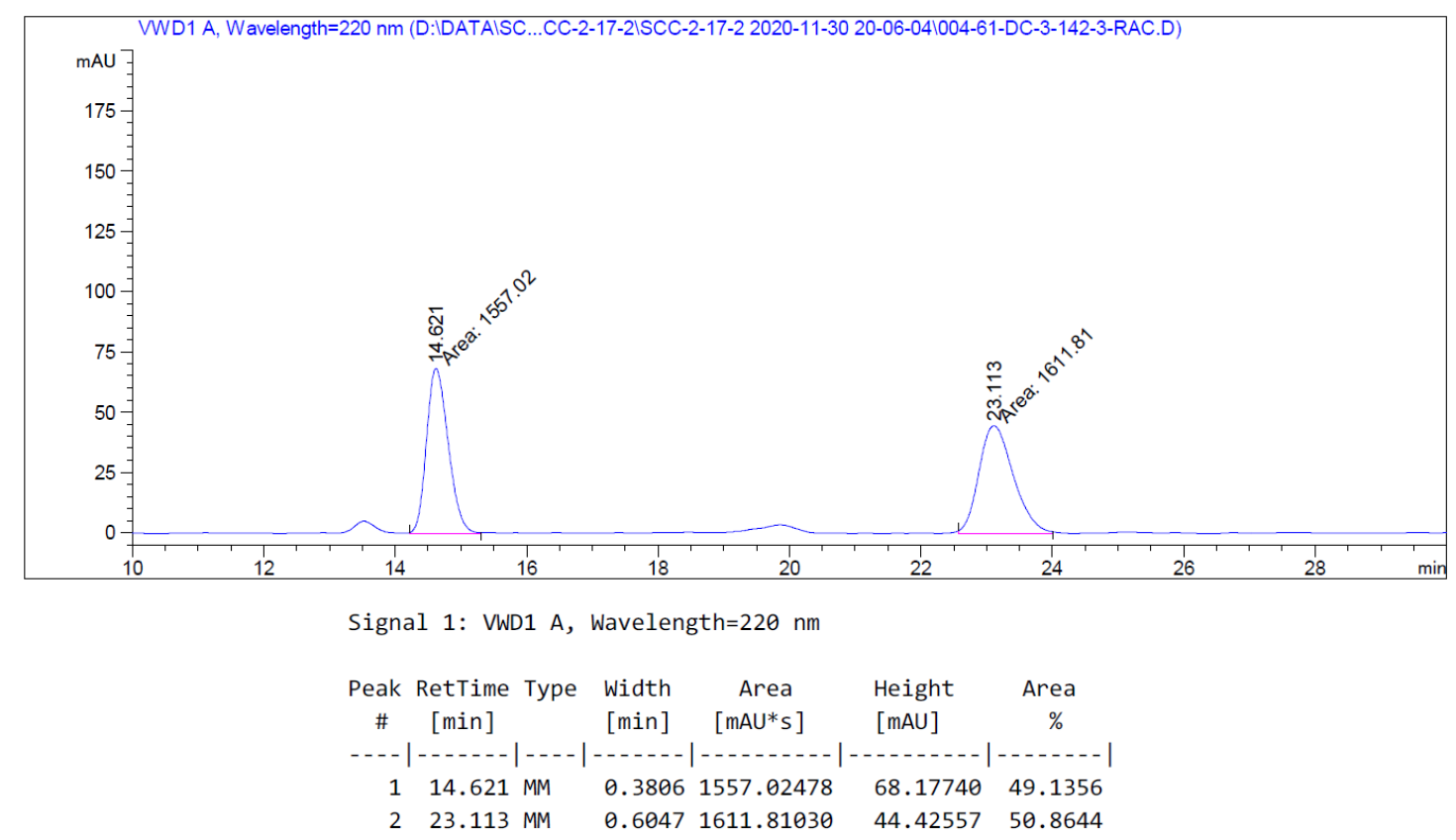



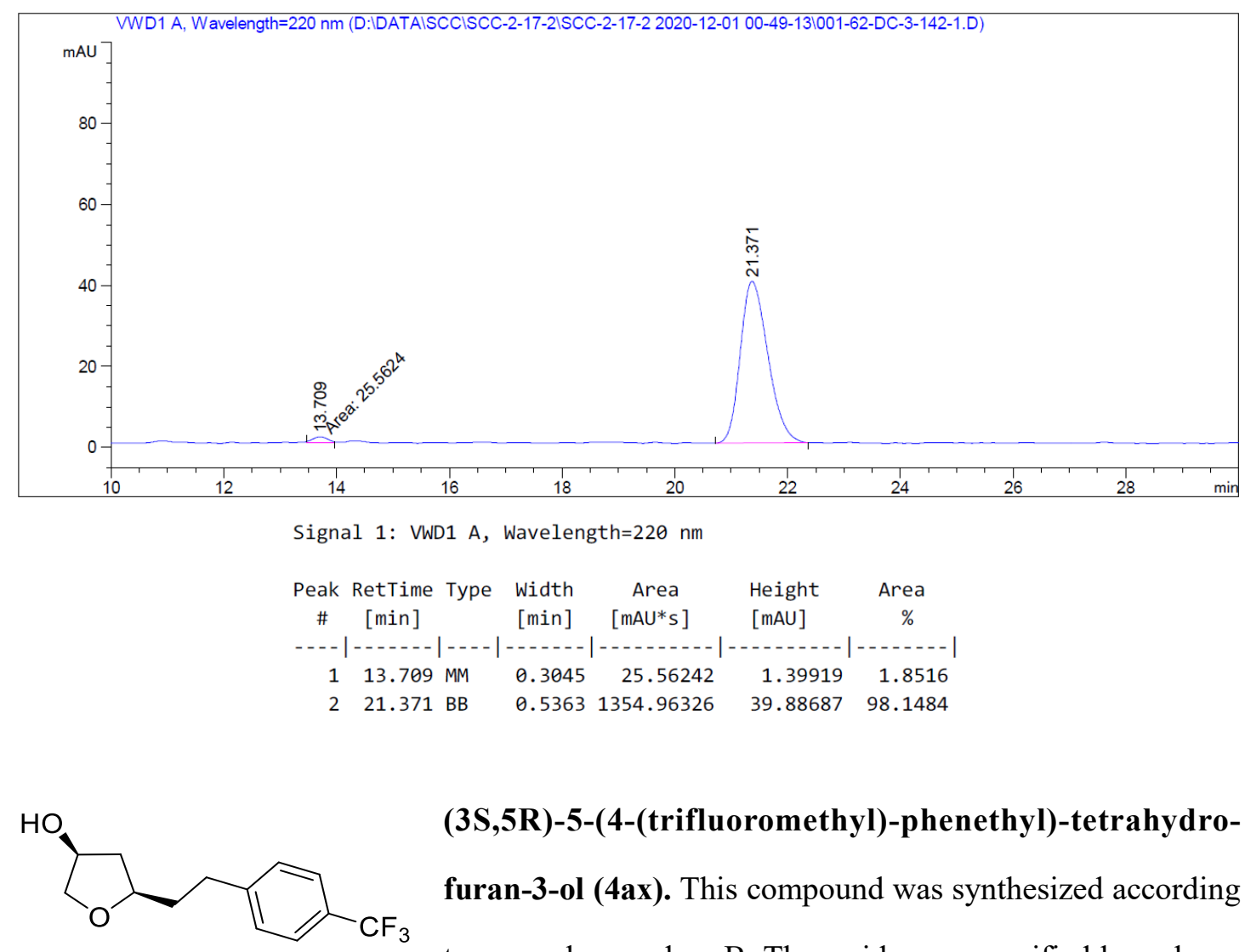

(3S,5R)-5-(4-(trifluoromethyl)-phenethyl)-tetrahydrofuran-3-ol (4ax). This compound was synthesized according to general procedure B. The residue was purified by column chromatography on silica gel to afford the product $4 \mathbf{a x}(78.0 \mathrm{mg}, 75 \%$ yield, $\mathrm{dr}>20: 1)$ as a colorless oil; ${ }^{1}$ H NMR (400 MHz, Chloroform- $d$ ) $\delta 7.53(\mathrm{~d}, J=8.0 \mathrm{~Hz}, 2 \mathrm{H}), 7.31$ (d, $J=8.0$ $\mathrm{Hz}, 2 \mathrm{H}), 4.54-4.40(\mathrm{~m}, 1 \mathrm{H}), 3.87(\mathrm{dt}, J=9.8,1.6 \mathrm{~Hz}, 1 \mathrm{H}), 3.80(\mathrm{td}, J=7.6,5.0 \mathrm{~Hz}, 1 \mathrm{H}), 3.70$ (dd, $J=9.9,4.4 \mathrm{~Hz}, 1 \mathrm{H}), 2.86$ (ddd, $J=15.1,9.9,5.6 \mathrm{~Hz}, 1 \mathrm{H}$ ), 2.74 (ddd, $J=13.9,9.6,6.6 \mathrm{~Hz}$, 1H), $2.34(\mathrm{dt}, J=14.0,7.2 \mathrm{~Hz}, 1 \mathrm{H}), 2.09-1.98(\mathrm{~m}, 2 \mathrm{H}), 1.94-1.85(\mathrm{~m}, 1 \mathrm{H}), 1.55$ (dddd, $J=$ 13.5, 7.1, 2.9, $1.4 \mathrm{~Hz}, 1 \mathrm{H}) \mathrm{ppm} ;{ }^{13} \mathbf{C}$ NMR (151 MHz, $\left.\mathbf{C D C l}_{3}\right) \delta 146.1,128.8,128.3(\mathrm{q}, J=$ $32.3 \mathrm{~Hz}), 125.4(\mathrm{q}, J=3.9 \mathrm{~Hz}), 124.5(\mathrm{q}, J=272.0 \mathrm{~Hz}), 78.2,75.6,72.6,41.4,37.6,32.5 \mathrm{ppm}$; ${ }^{19}$ F NMR (376 MHz, CDCl3) $\delta-62.17$ ppm; HRMS (ESI) calculated $[\mathrm{M}+\mathrm{H}]^{+}$for $\mathrm{C}_{13} \mathrm{H}_{16} \mathrm{~F}_{3} \mathrm{O}_{2}{ }^{+}$ $=261.1097$, found: $261.1097 .[\alpha]^{20}{ }_{\mathbf{D}}=+7.3\left(\mathrm{c}=0.44, \mathrm{CHCl}_{3}\right)$. HPLC analysis $(\mathrm{OD}-\mathrm{H}, n-$ Hexane: $i$-Propanol $=97: 3$ as eluent, $1 \mathrm{~mL} / \mathrm{min}, 220 \mathrm{~nm})$ indicated $96 \%$ ee: $\mathrm{t}_{\mathrm{R}}($ minor $)=23.9$ $\min , t_{R}($ major $)=26.1 \min$. 


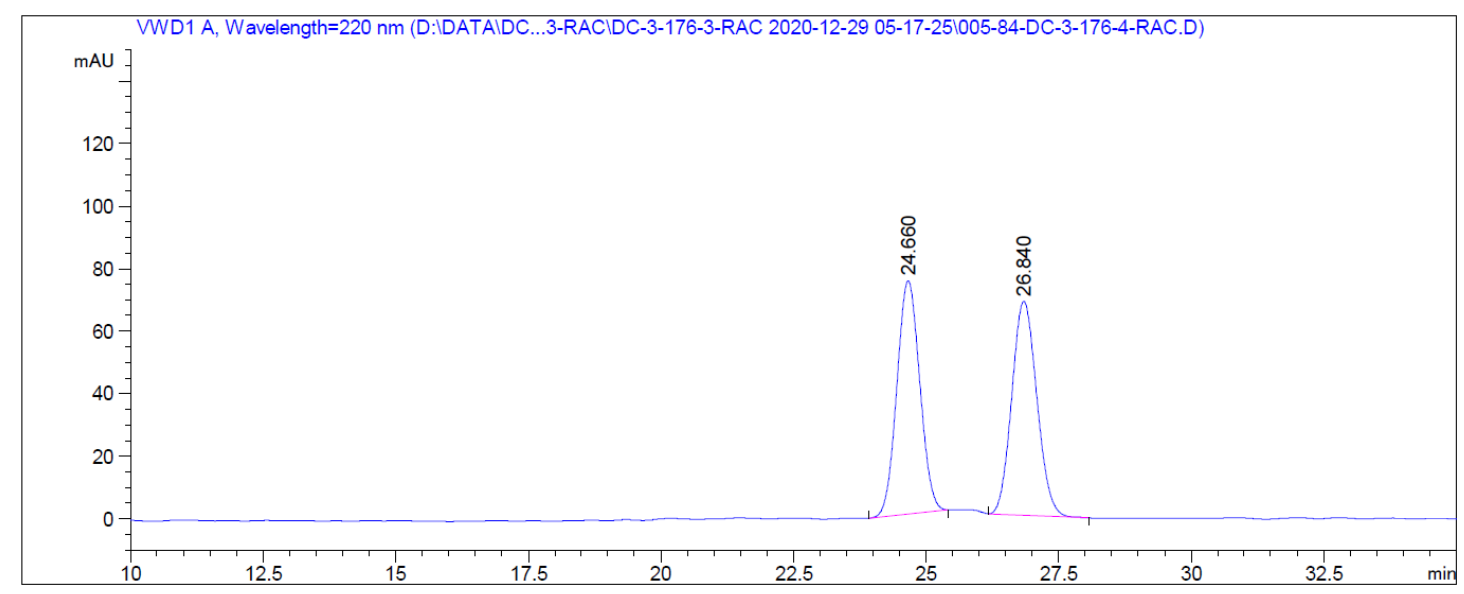

Signal 1: VWD1 A, Wavelength $=220 \mathrm{~nm}$
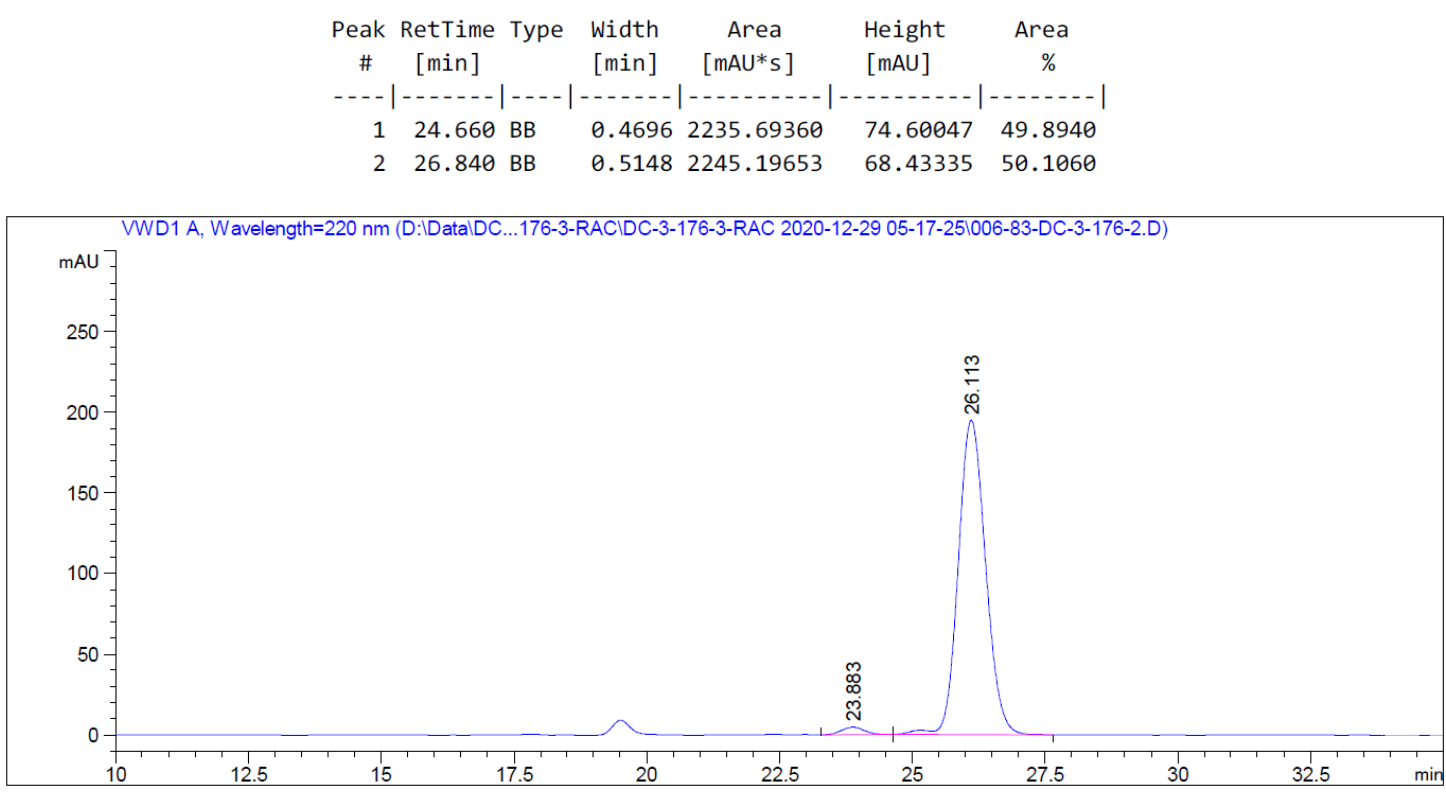

Signal 1: VWD1 A, Wavelength $=220 \mathrm{~nm}$
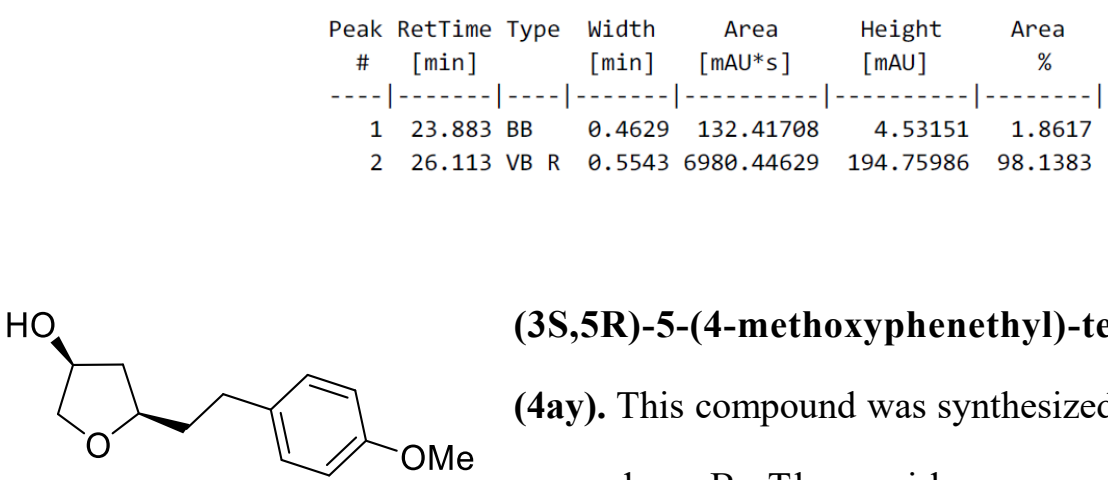

(3S,5R)-5-(4-methoxyphenethyl)-tetrahydrofuran-3-ol

(4ay). This compound was synthesized according to general

procedure B. The residue was purified by column chromatography on silica gel to afford the product 4 ay $(58.0 \mathrm{mg}, 65 \%$ yield, $\mathrm{dr}=17: 1)$ as a white solid; ${ }^{1} \mathbf{H}$ NMR (400 MHz, Chloroform- $d$ ) $\delta 7.12$ (d, $\left.J=8.6 \mathrm{~Hz}, 2 \mathrm{H}\right), 6.82$ (d, $J=8.6$ $\mathrm{Hz}, 2 \mathrm{H}), 4.49-4.39(\mathrm{~m}, 1 \mathrm{H}), 3.85(\mathrm{dt}, J=10.0,1.6 \mathrm{~Hz}, 1 \mathrm{H}), 3.82-3.76(\mathrm{~m}, 4 \mathrm{H}), 3.68(\mathrm{dd}, J=$ 9.9, 4.4 Hz, 1H), $2.77-2.68(\mathrm{~m}, 1 \mathrm{H}), 2.67-2.54(\mathrm{~m}, 1 \mathrm{H}), 2.33(\mathrm{dt}, J=13.3,7.2 \mathrm{~Hz}, 1 \mathrm{H}), 2.01$ 
(dddd, $J=13.3,9.5,7.6,5.8 \mathrm{~Hz}, 1 \mathrm{H}), 1.95-1.88(\mathrm{~m}, 1 \mathrm{H}), 1.87-1.75(\mathrm{~m}, 1 \mathrm{H}), 1.52$ (dddd, $J=$ 13.5, 7.2, 2.9, $1.4 \mathrm{~Hz}, 1 \mathrm{H}) \mathrm{ppm} ;{ }^{13} \mathbf{C}$ NMR (151 MHz, $\left.\mathbf{C D C l}_{3}\right) \delta 157.9,134.0,129.4,113.9$, 78.4, 75.5, 72.7, 55.4, 41.5, 38.2, 31.7 ppm; HRMS (ESI) calculated $[\mathrm{M}+\mathrm{H}]^{+}$for $\mathrm{C}_{13} \mathrm{H}_{19} \mathrm{O}_{3}{ }^{+}=$ 223.1329, found: $223.1328 .[\alpha]^{20}{ }_{\mathbf{D}}=+4.8\left(\mathrm{c}=0.18, \mathrm{CHCl}_{3}\right)$. HPLC analysis (OD-H, $n$-Hexane: $i$-Propanol $=95: 5$ as eluent, $1 \mathrm{~mL} / \mathrm{min}, 220 \mathrm{~nm}$ ) indicated 95\% ee: $\mathrm{t}_{\mathrm{R}}($ minor $)=16.3 \mathrm{~min}$, $\mathrm{t}_{\mathrm{R}}($ major $)=19.3 \mathrm{~min}$.

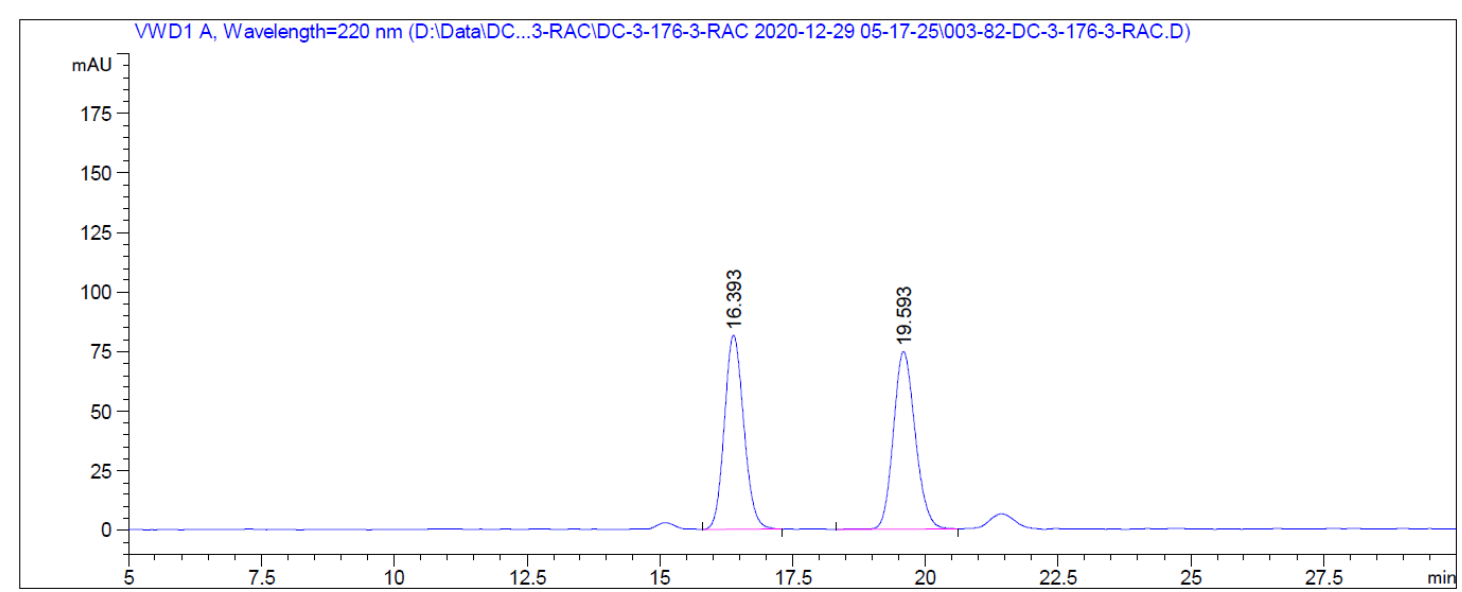

Signal 1: VWD1 A, Wavelength $=220 \mathrm{~nm}$
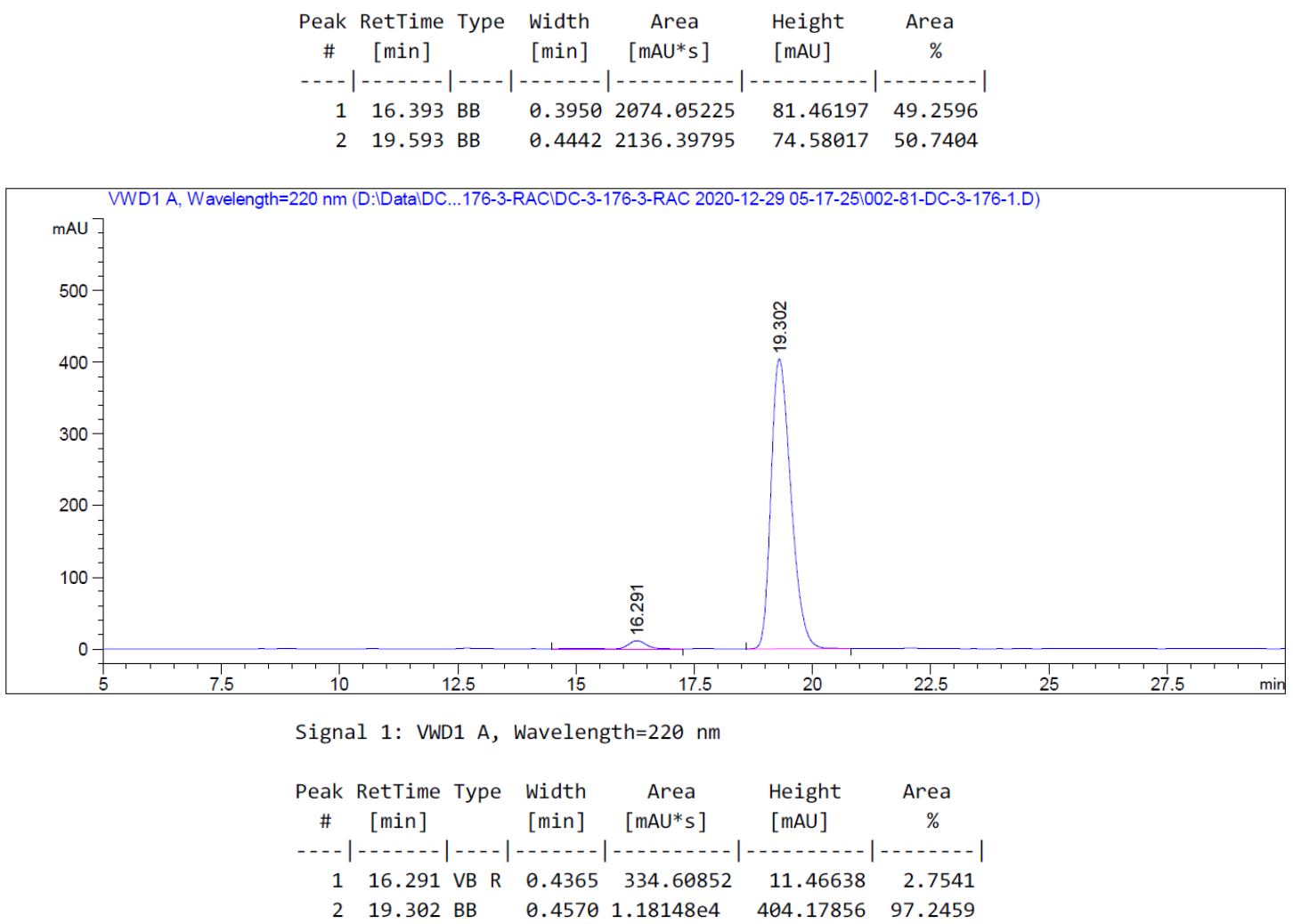


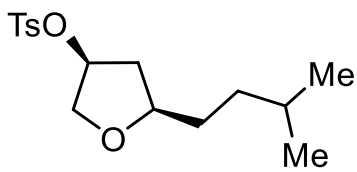

(3S,5R)-5-isopentyltetrahydrofuran-3-yl 4-methylbenzenesulf-

onate (4az). This compound was synthesized according to general procedure B and protected with Tosyl chloride according to reported

literature. ${ }^{3}$ The residue was purified by column chromatography on silica gel to afford the product 4az (41.1 mg, 60\% yield, dr > 20:1) as a colorless oil; ${ }^{1} \mathbf{H}$ NMR (400 MHz, Chloroform- $d$ ) $\delta 7.79(\mathrm{~d}, J=8.4 \mathrm{~Hz}, 2 \mathrm{H}), 7.35(\mathrm{~d}, J=8.2 \mathrm{~Hz}, 2 \mathrm{H}), 5.07$ (dddd, $J=7.7,4.9$, $3.2,1.8 \mathrm{~Hz}, 1 \mathrm{H}), 3.96(\mathrm{dt}, J=11.0,1.6 \mathrm{~Hz}, 1 \mathrm{H}), 3.77-3.69(\mathrm{~m}, 1 \mathrm{H}), 3.66(\mathrm{dd}, J=11.0,4.8$ $\mathrm{Hz}, 1 \mathrm{H}), 2.45(\mathrm{~s}, 3 \mathrm{H}), 2.31(\mathrm{dt}, J=14.2,7.2 \mathrm{~Hz}, 1 \mathrm{H}), 1.76-1.70(\mathrm{~m}, 1 \mathrm{H}), 1.67-1.60(\mathrm{~m}, 1 \mathrm{H})$, $1.57-1.44(\mathrm{~m}, 2 \mathrm{H}), 1.27$ (dddd, $J=13.3,11.5,6.7,5.0 \mathrm{~Hz}, 1 \mathrm{H}), 1.12($ dddd, $J=13.2,11.7$, $6.8,5.1 \mathrm{~Hz}, 1 \mathrm{H}), 0.87(\mathrm{~d}, J=6.6 \mathrm{~Hz}, 6 \mathrm{H}) \mathrm{ppm} ;{ }^{13} \mathbf{C} \mathbf{N M R}\left(\mathbf{1 0 1} \mathbf{M H z}, \mathbf{C D C l}_{3}\right) \delta 145.0,134.0$, 130.0, 127.9, 81.7, 79.4, 72.4, 38.4, 35.2, 33.2, 28.1, 22.6, 21.8 ppm; HRMS (ESI) calculated $[\mathrm{M}+\mathrm{H}]^{+}$for $\mathrm{C}_{16} \mathrm{H}_{25} \mathrm{O}_{4} \mathrm{~S}^{+}=313.1468$, found: $313.1460 .[\mathbf{\alpha}]^{20} \mathbf{D}=-9.1\left(\mathrm{c}=0.13, \mathrm{CHCl}_{3}\right) . \mathbf{H P L C}$ analysis $(\mathrm{AD}-\mathrm{H}, n$-Hexane: $i$-Propanol $=95: 5$ as eluent, $1 \mathrm{~mL} / \mathrm{min}, 220 \mathrm{~nm})$ indicated $95 \%$ ee: $t_{R}($ minor $)=8.8$ min, $t_{R}($ major $)=9.6 \mathrm{~min}$.

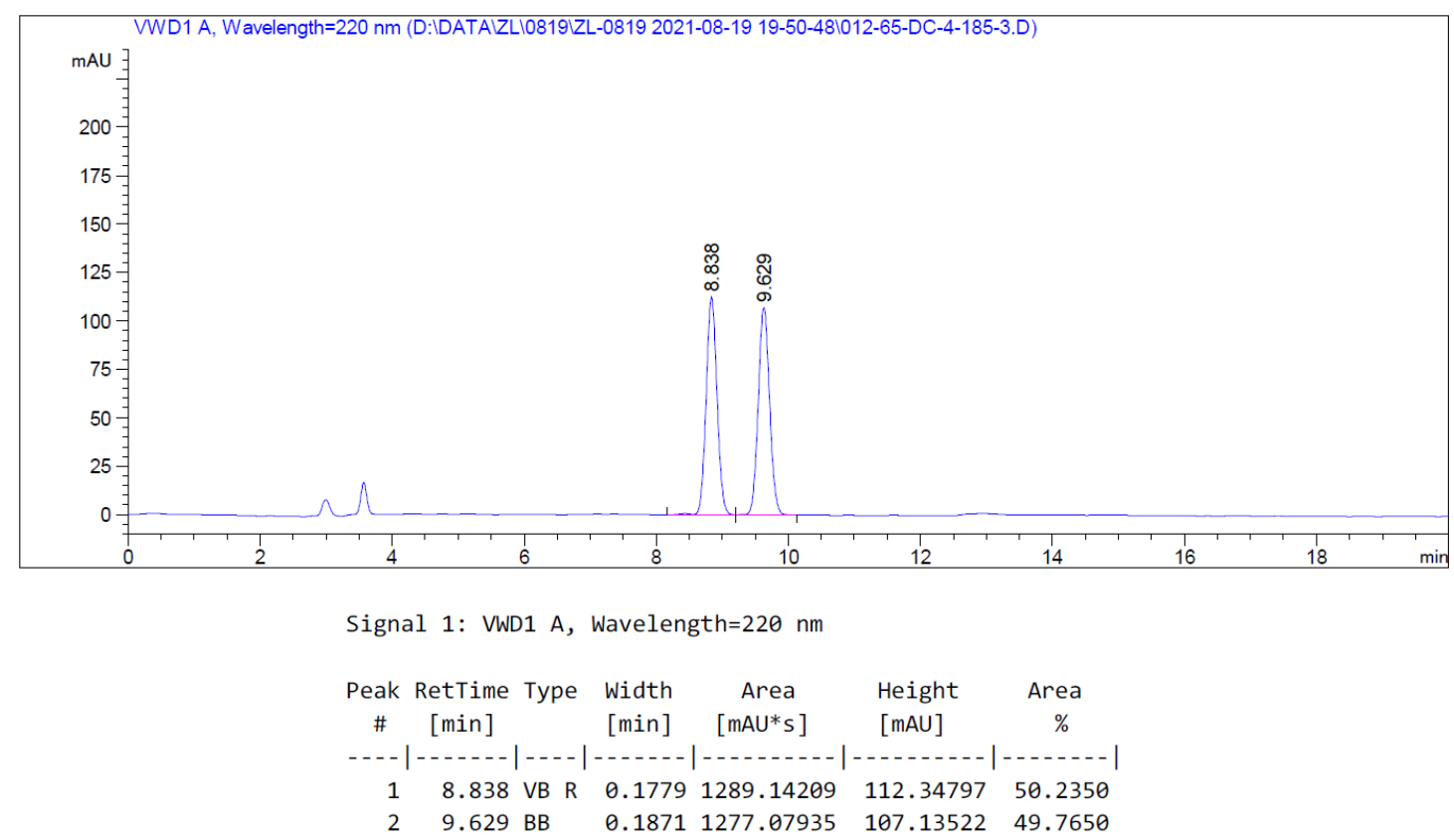



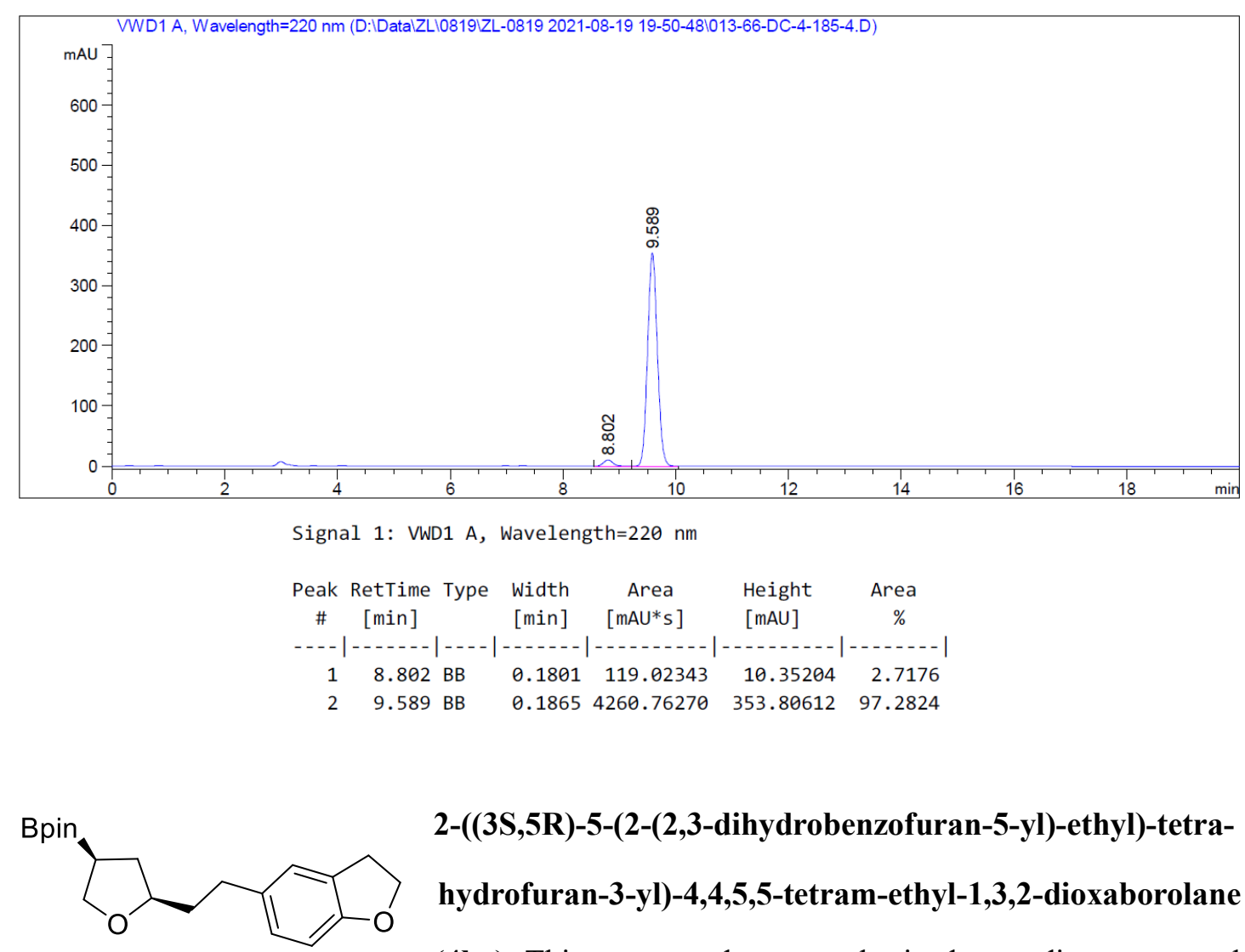

2-((3S,5R)-5-(2-(2,3-dihydrobenzofuran-5-yl)-ethyl)-tetrahydrofuran-3-yl)-4,4,5,5-tetram-ethyl-1,3,2-dioxaborolane

(4ba). This compound was synthesized according to general procedure $\mathrm{B}$. The residue was purified by column chromatography on silica gel to afford the product $4 \mathbf{b a}(101.0 \mathrm{mg}, 74 \%$ yield, $\mathrm{dr}=17: 1)$ as a colorless oil; ${ }^{1} \mathbf{H}$ NMR $(600 \mathrm{MHz}$, Chloroform-d) $\delta 7.03(\mathrm{~d}, J=1.9 \mathrm{~Hz}, 1 \mathrm{H}), 6.94-6.87(\mathrm{~m}, 1 \mathrm{H}), 6.68(\mathrm{~d}, J=8.1 \mathrm{~Hz}, 1 \mathrm{H}), 4.53$ (t, $J=8.6 \mathrm{~Hz}, 2 \mathrm{H}), 3.97$ (t, $J=8.4 \mathrm{~Hz}, 1 \mathrm{H}), 3.83-3.77(\mathrm{~m}, 2 \mathrm{H}), 3.16(\mathrm{t}, J=8.6 \mathrm{~Hz}, 2 \mathrm{H}), 2.67$ (ddd, $J=13.8,10.1,5.7 \mathrm{~Hz}, 1 \mathrm{H}), 2.58$ (ddd, $J=13.8,10.0,6.2 \mathrm{~Hz}, 1 \mathrm{H}), 2.14$ (ddd, $J=12.1$, 7.8, $5.5 \mathrm{~Hz}, 1 \mathrm{H}), 1.88$ (dddd, $J=13.2,10.0,7.3,5.7 \mathrm{~Hz}, 1 \mathrm{H}), 1.72$ (dddd, $J=25.6,11.6,9.8$, $6.9 \mathrm{~Hz}, 2 \mathrm{H}), 1.46$ (td, $J=11.7,9.1 \mathrm{~Hz}, 1 \mathrm{H}), 1.24(\mathrm{~s}, 12 \mathrm{H}) \mathrm{ppm} ;{ }^{13} \mathbf{C}$ NMR (151 $\left.\mathbf{M H z}, \mathbf{C D C l}_{\mathbf{3}}\right)$ $\delta 158.2,134.3,127.8,127.0,125.0,109.0,83.5,79.7,71.2,69.7,37.9,35.0,32.3,29.9,24.8$ ppm; ${ }^{11}$ B NMR (128 MHz, Chloroform- $d$ ) $\delta 33.52$ ppm; HRMS (ESI) calculated $\left.[\mathrm{M}+\mathrm{H}]\right]^{+}$for $\mathrm{C}_{20} \mathrm{H}_{30} \mathrm{BO}_{4}{ }^{+}=345.2232$, found: $345.2224 .[\alpha]^{20}{ }_{\mathbf{D}}=+2.3\left(\mathrm{c}=0.57, \mathrm{CHCl}_{3}\right)$, the corresponding alcohol after oxidation. HPLC analysis (OD-H, $n$-Hexane: $i$-Propanol $=95: 5$ as eluent, 1 $\mathrm{mL} / \mathrm{min}, 220 \mathrm{~nm}$ ) indicated 95\% ee: $\mathrm{t}_{\mathrm{R}}($ minor $)=23.0 \mathrm{~min}, \mathrm{t}_{\mathrm{R}}($ major $)=27.2 \mathrm{~min}$. 


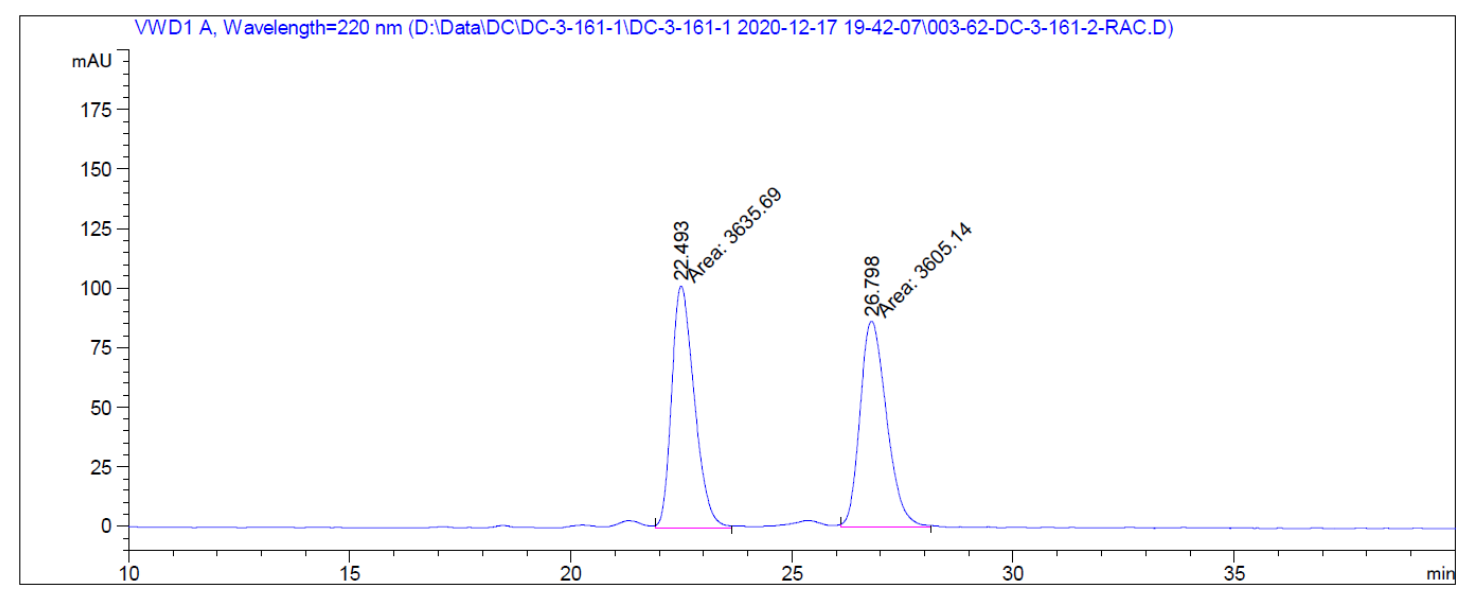

Signal 1: VWD1 A, Wavelength $=220 \mathrm{~nm}$
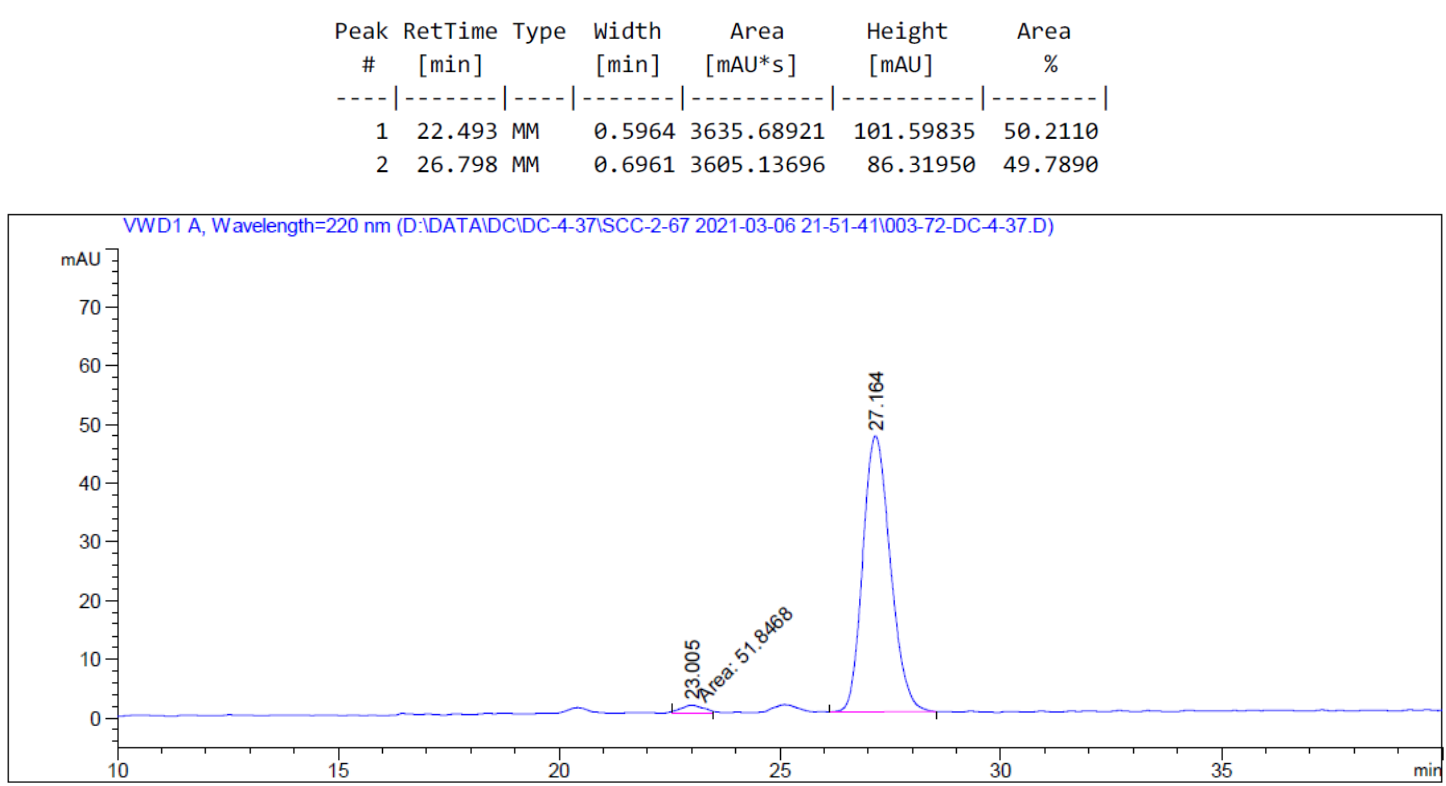

Signal 1: VWD1 A, Wavelength $=220 \mathrm{~nm}$
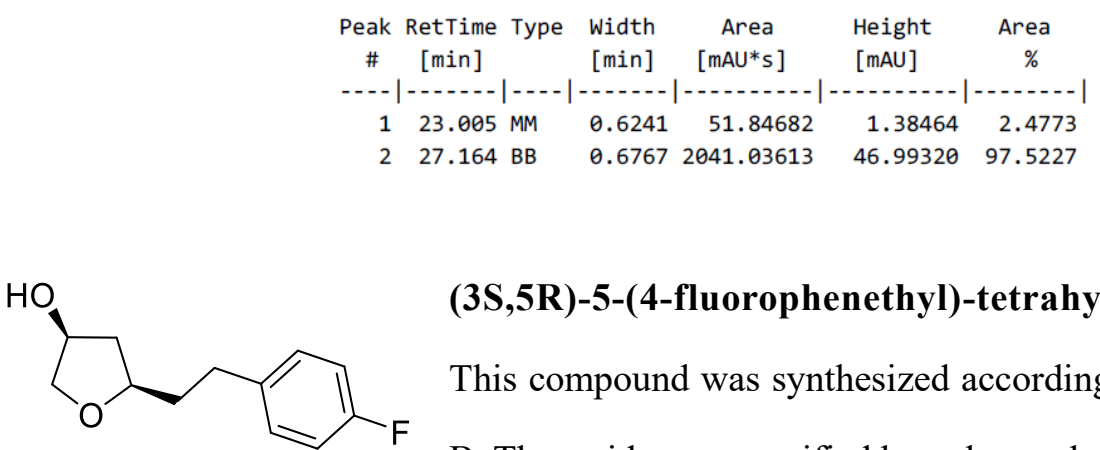

(3S,5R)-5-(4-fluorophenethyl)-tetrahydrofuran-3-ol(4bb).

This compound was synthesized according to general procedure

B. The residue was purified by column chromatography on silica gel to afford the product $\mathbf{4 b b}(41.0 \mathrm{mg}, 49 \%$ yield, $\mathrm{dr}>20: 1)$ as a colorless oil; ${ }^{1} \mathbf{H}$ NMR (600

MHz, Chloroform-d) $\delta 7.18$ - $7.11(\mathrm{~m}, 2 \mathrm{H}), 6.98$ - $6.93(\mathrm{~m}, 2 \mathrm{H}), 4.52$ - $4.39(\mathrm{~m}, 1 \mathrm{H}), 3.86(\mathrm{dt}$, $J=9.9,1.6 \mathrm{~Hz}, 1 \mathrm{H}), 3.80(\mathrm{qd}, J=7.5,5.1 \mathrm{~Hz}, 1 \mathrm{H}), 3.69(\mathrm{dd}, J=9.9,4.4 \mathrm{~Hz}, 1 \mathrm{H}), 2.80-2.73$ (m, 1H), $2.69-2.61(\mathrm{~m}, 1 \mathrm{H}), 2.34(\mathrm{ddd}, J=13.4,7.7,6.7 \mathrm{~Hz}, 1 \mathrm{H}), 2.05-1.96(\mathrm{~m}, 1 \mathrm{H}), 1.89$ - 
$1.81(\mathrm{~m}, 1 \mathrm{H}), 1.77(\mathrm{~s}, 1 \mathrm{H}), 1.54(\mathrm{dddd}, J=13.5,7.1,2.8,1.4 \mathrm{~Hz}, 1 \mathrm{H}) \mathrm{ppm} ;{ }^{13} \mathbf{C}$ NMR $(\mathbf{1 5 1}$

MHz, $\left.\mathbf{C D C l}_{3}\right) \delta 161.4(\mathrm{~d}, J=243.2 \mathrm{~Hz}), 137.5(\mathrm{~d}, J=3.3 \mathrm{~Hz}), 129.9(\mathrm{~d}, J=7.8 \mathrm{~Hz}), 115.2(\mathrm{~d}$, $J=21.2 \mathrm{~Hz}), 78.3,75.6,72.7,41.5,38.1,31.9$ ppm; 19F NMR (376 MHz, Chloroform-d) $\delta$ -177.76 ppm; HRMS (ESI) calculated $[\mathrm{M}+\mathrm{H}]^{+}$for $\mathrm{C}_{12} \mathrm{H}_{16} \mathrm{FO}_{2}{ }^{+}=211.1129$, found: 211.1128 $[\boldsymbol{\alpha}]^{20}{ }_{\mathbf{D}}=+7.1\left(\mathrm{c}=0.20, \mathrm{CHCl}_{3}\right)$. HPLC analysis $(\mathrm{OD}-\mathrm{H}, n$-Hexane: $i$-Propanol $=95: 5$ as eluent, $1 \mathrm{~mL} / \mathrm{min}, 254 \mathrm{~nm})$ indicated $94 \%$ ee: $t_{R}($ minor $)=12.1 \mathrm{~min}, t_{R}($ major $)=15.2 \mathrm{~min}$.

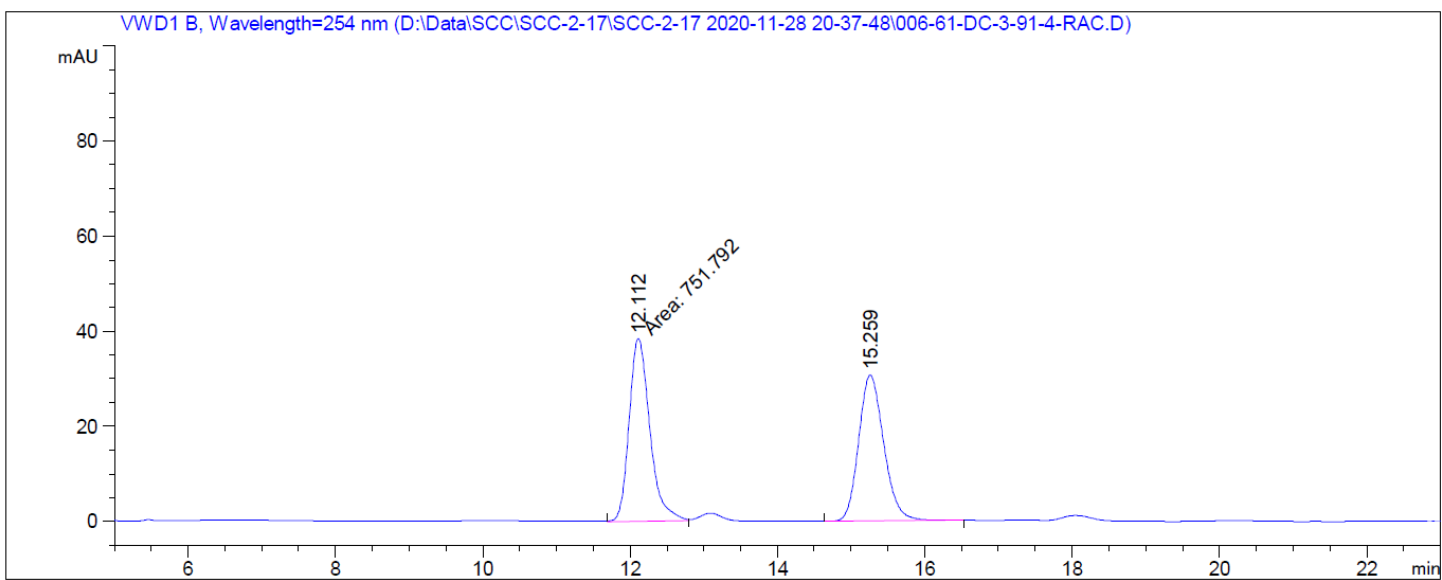

Signal 1: VWD1 B, Wavelength $=254 \mathrm{~nm}$

\begin{tabular}{cccccc}
$\begin{array}{c}\text { Peak RetTime Type } \\
\text { Width } \\
\text { [min] }\end{array}$ & $\begin{array}{c}\text { Area } \\
{[\text { min] }}\end{array}$ & $\begin{array}{c}\text { Height } \\
{[\mathrm{mAU} \text { s }]}\end{array}$ & $\begin{array}{c}\text { Area } \\
{[\mathrm{mAU}]}\end{array}$ & $\%$ \\
\hline 1 & 12.112 MM & 0.3257 & 751.79181 & 38.46867 & 50.7869 \\
2 & 15.259 BB & 0.3671 & 728.49567 & 30.67688 & 49.2131
\end{tabular}

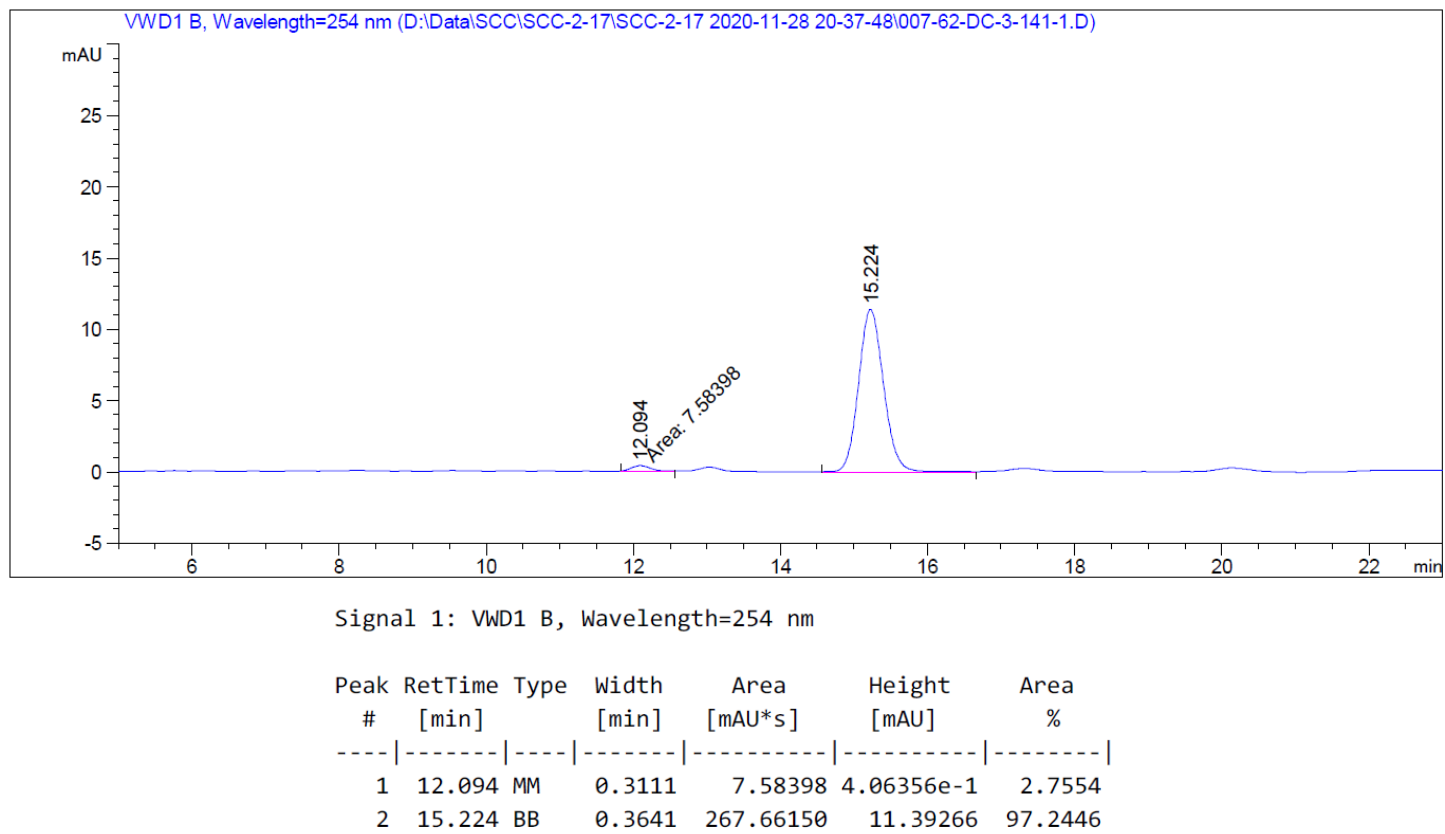




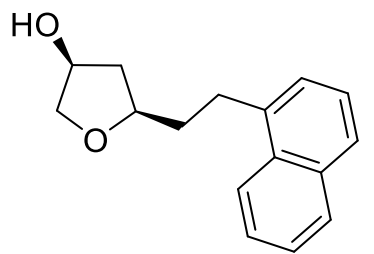

(3S,5R)-5-(2-(naphthalen-1-yl)-ethyl)-tetrahydrofuran-3-ol (4bc).

This compound was synthesized according to general procedure B.

The residue was purified by column chromatography on silica gel to afford the product $\mathbf{4 b c}(63.0 \mathrm{mg}, 65 \%$ yield, $\mathrm{dr}>20: 1)$ as a colorless oil; ${ }^{1} \mathrm{H}$ NMR (600 MHz, Chloroform-d) $\delta 8.06(\mathrm{dd}, J=8.4,1.1 \mathrm{~Hz}, 1 \mathrm{H}), 7.85-7.82(\mathrm{~m}, 1 \mathrm{H})$, $7.70(\mathrm{dt}, J=8.1,1.1 \mathrm{~Hz}, 1 \mathrm{H}), 7.48$ (dddd, $J=24.0,8.1,6.8,1.4 \mathrm{~Hz}, 2 \mathrm{H}), 7.40-7.33(\mathrm{~m}, 2 \mathrm{H})$, $4.49-4.39(\mathrm{~m}, 1 \mathrm{H}), 3.92-3.85(\mathrm{~m}, 2 \mathrm{H}), 3.71(\mathrm{dd}, J=9.9,4.5 \mathrm{~Hz}, 1 \mathrm{H}), 3.32-3.23(\mathrm{~m}, 1 \mathrm{H})$, $3.15-3.08$ (m, 1H), 2.34 (ddd, $J=13.4,7.6,6.8 \mathrm{~Hz}, 1 \mathrm{H}), 2.15$ (dddd, $J=13.4,9.9,7.9,5.4$ Hz, 1H), 2.01 (dddd, $J=13.7,10.0,6.4,4.9 \mathrm{~Hz}, 1 \mathrm{H}), 1.95$ (s, 1H), 1.54 (dddd, $J=13.5,7.2$, 3.0, $1.3 \mathrm{~Hz}, 1 \mathrm{H}) \mathrm{ppm} ;{ }^{13} \mathbf{C} \mathbf{N M R}\left(\mathbf{1 5 1} \mathbf{M H z}, \mathbf{C D C l}_{3}\right) \delta 137.1,133.0,131.0,127.9,125.8,125.1$, 125.0, 124.7 124.6, 122.9, 77.7, 74.6, 71.6, 40.5, 36.2, 28.8 ppm; HRMS (ESI) calculated $[\mathrm{M}+\mathrm{H}]^{+}$for $\mathrm{C}_{16} \mathrm{H}_{19} \mathrm{O}_{2}{ }^{+}=243.1380$, found: $243.1379 .[\boldsymbol{\alpha}]^{20} \mathbf{D}=+14.0\left(\mathrm{c}=0.46, \mathrm{CHCl}_{3}\right) . \mathbf{H P L C}$ analysis (OD-H, $n$-Hexane: $i$-Propanol $=95: 5$ as eluent, $1 \mathrm{~mL} / \mathrm{min}, 220 \mathrm{~nm}$ ) indicated $96 \%$ ee: $t_{R}($ minor $)=30.8 \mathrm{~min}, t_{R}($ major $)=43.0 \mathrm{~min}$.

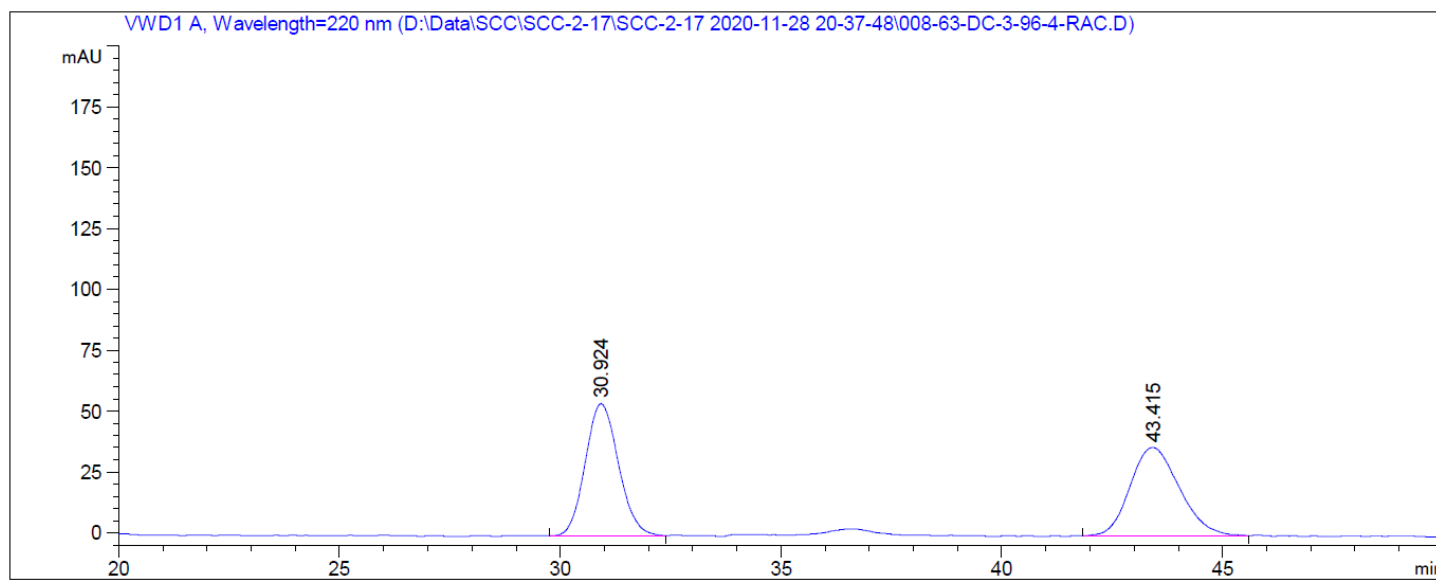

Signal 1: VWD1 A, Wavelength $=220 \mathrm{~nm}$

\begin{tabular}{cccccc}
$\begin{array}{c}\text { Peak RetTime Type } \\
\text { \# } \\
\text { [min] }\end{array}$ & $\begin{array}{c}\text { Width } \\
\text { [min] }\end{array}$ & $\begin{array}{c}\text { Area } \\
{[\mathrm{mAU} \text { s }]}\end{array}$ & $\begin{array}{c}\text { Height } \\
\text { [mAU] }\end{array}$ & \multicolumn{1}{c}{$\begin{array}{c}\text { Area } \\
\%\end{array}$} \\
\hline 1 & 3.016 BB & 0.1168 & 62.97482 & 8.08802 & 1.1057 \\
2 & 30.924 BB & 0.8186 & 2828.89697 & 54.22546 & 49.6678 \\
3 & 43.415 BB & 1.1631 & 2803.76392 & 36.22215 & 49.2265
\end{tabular}




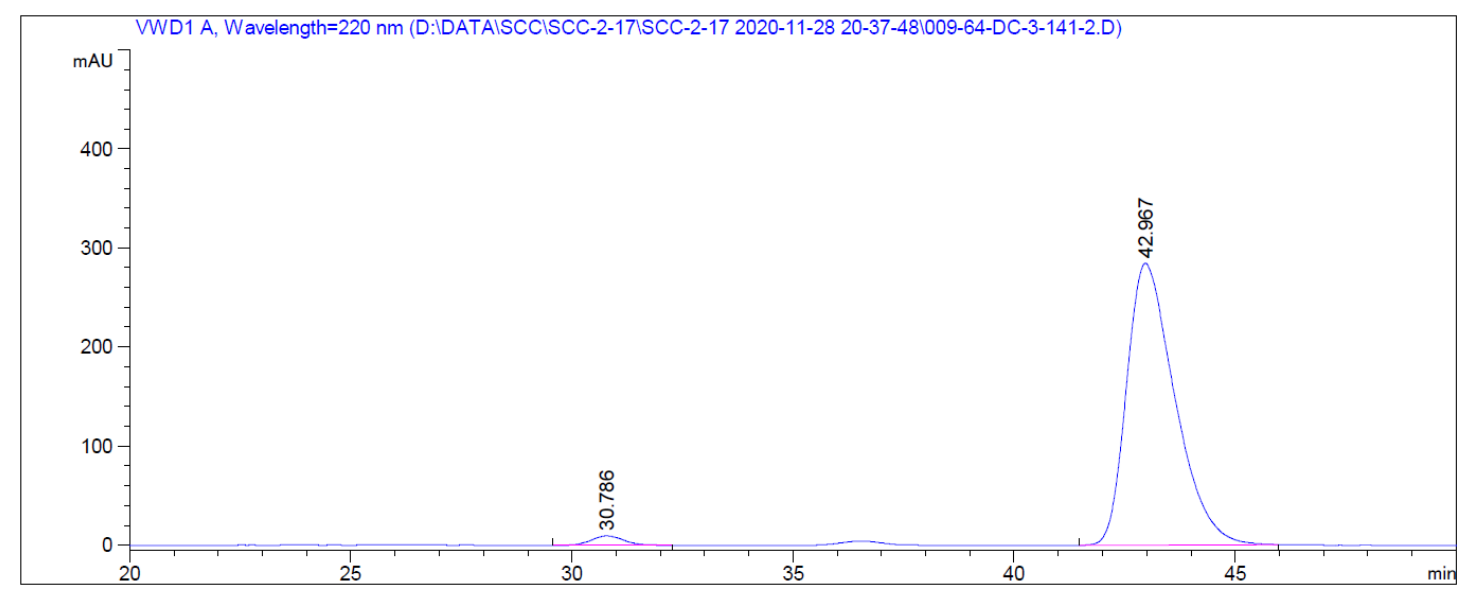

Signal 1: VWD1 A, Wavelength $=220 \mathrm{~nm}$

\begin{tabular}{|c|c|c|c|c|c|c|}
\hline $\begin{array}{c}\text { Peak } \\
\quad \#\end{array}$ & $\begin{array}{c}\text { RetTime } \\
\text { [min] }\end{array}$ & Type & $\begin{array}{l}\text { Width } \\
\text { [min] }\end{array}$ & $\begin{array}{c}\text { Area } \\
{\left[\mathrm{mAU}^{*} \mathrm{~s}\right]}\end{array}$ & $\begin{array}{l}\text { Height } \\
{[\mathrm{mAU}]}\end{array}$ & $\begin{array}{c}\text { Area } \\
\quad \%\end{array}$ \\
\hline & & & & | & & $-----\mid$ \\
\hline 1 & 30.786 & BB & 0.8058 & 498.19092 & 9.56297 & 2.2612 \\
\hline 2 & 42.967 & BB & 1.1228 & $2.15343 \mathrm{e} 4$ & 284.59238 & 97.7388 \\
\hline
\end{tabular}

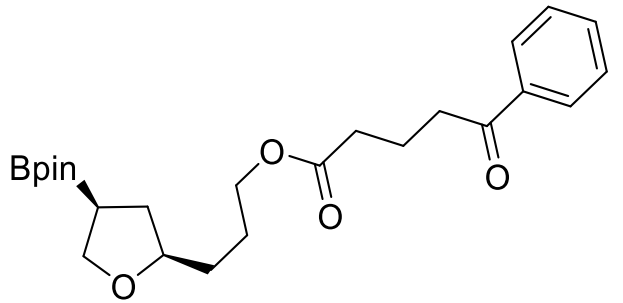

3-((2R,4S)-4-(4,4,5,5-tetramethyl-1,3,2-dioxaborolan-2-yl)-tetrahydrofuran-2-yl)-propyl-5-oxo-5phenylpentanoate (4bd). This compound was synthesized according to general procedure B. The residue was purified by column chromatography on silica gel to afford the product $\mathbf{4 b d}(91.0$ mg, 53\% yield, $\mathrm{dr}=16: 1)$ as a colorless oil; ${ }^{1} \mathbf{H}$ NMR (400 MHz, Chloroform- $\left.\boldsymbol{d}\right) \delta 7.97-7.89$ (m, 2H), $7.58-7.50(\mathrm{~m}, 1 \mathrm{H}), 7.48-7.38(\mathrm{~m}, 2 \mathrm{H}), 4.08(\mathrm{td}, J=6.5,1.5 \mathrm{~Hz}, 2 \mathrm{H}), 3.94(\mathrm{t}, J=8.4$ $\mathrm{Hz}, 1 \mathrm{H}), 3.83-3.71(\mathrm{~m}, 2 \mathrm{H}), 3.04(\mathrm{t}, J=7.2 \mathrm{~Hz}, 2 \mathrm{H}), 2.42(\mathrm{t}, J=7.2 \mathrm{~Hz}, 2 \mathrm{H}), 2.17-1.97(\mathrm{~m}$, $3 \mathrm{H}), 1.81-1.64(\mathrm{~m}, 3 \mathrm{H}), 1.61-1.48(\mathrm{~m}, 2 \mathrm{H}), 1.41(\mathrm{td}, J=11.8,9.2 \mathrm{~Hz}, 1 \mathrm{H}), 1.23(\mathrm{~s}, 12 \mathrm{H})$ ppm; ${ }^{13}$ C NMR (101 MHz, $\left.\mathbf{C D C l}_{3}\right) \delta$ 199.6, 173.5, 136.9, 133.2, 128.7, 128.2, 83.5, 79.9, 69.8, 64.6, 37.6, 35.1, 33.5, 31.9, 25.8, 24.9, 19.5 ppm; ${ }^{11}$ B NMR (128 MHz, Chloroform-d) $\delta 32.70$ ppm; HRMS (ESI) calculated $[\mathrm{M}+\mathrm{H}]^{+}$for $\mathrm{C}_{24} \mathrm{H}_{36} \mathrm{BO}_{6}{ }^{+}=431.2600$, found: $431.2594 .[\mathbf{\alpha}]^{\mathbf{2 0}} \mathbf{D}=$ $+11.5\left(\mathrm{c}=0.05, \mathrm{CHCl}_{3}\right)$, the corresponding alcohol after oxidation. HPLC analysis (OD-H, $n$ Hexane: $i$-Propanol $=90: 10$ as eluent, $1 \mathrm{~mL} / \mathrm{min}, 254 \mathrm{~nm}$ ) indicated $95 \%$ ee: $\mathrm{t}_{\mathrm{R}}($ minor $)=36.9$ $\min , t_{R}($ major $)=40.7 \min$. 


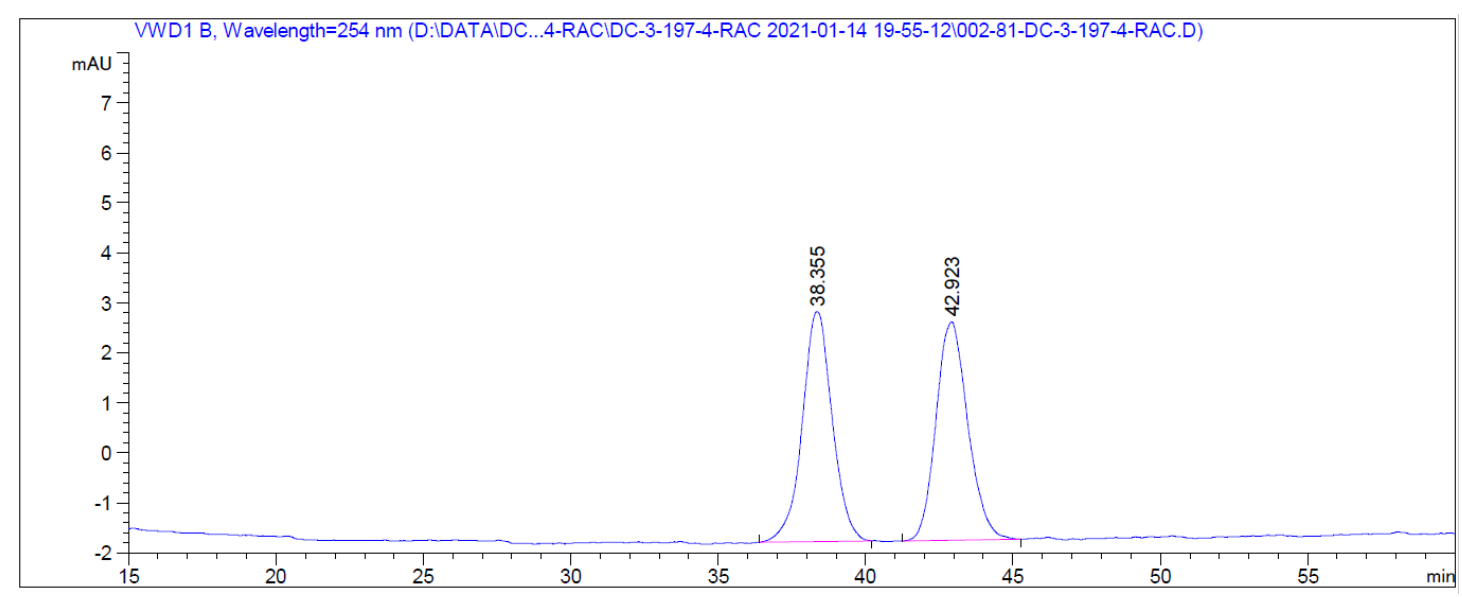

Signal 1: VWD1 B, Wavelength $=254 \mathrm{~nm}$
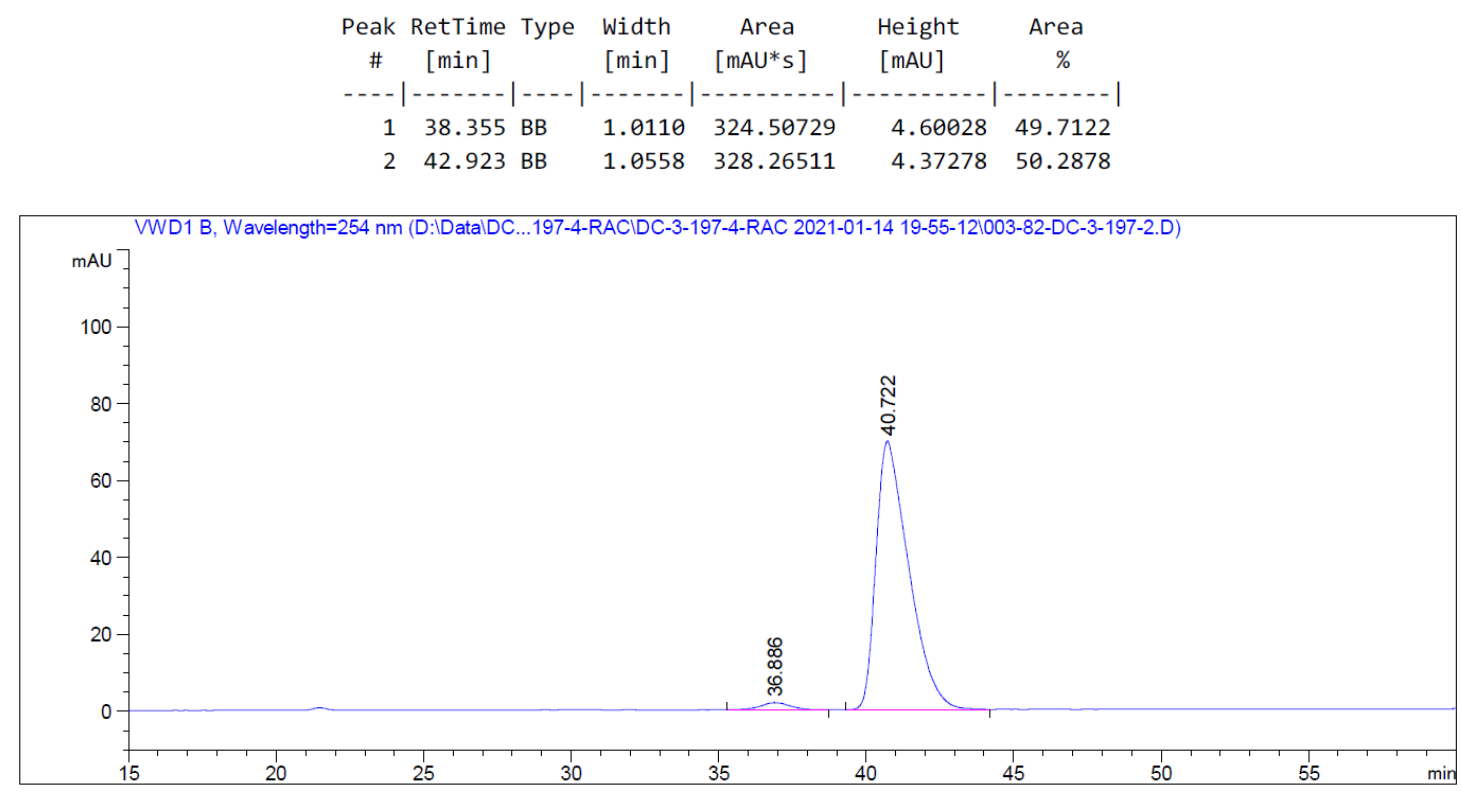

Signal 1: VWD1 B, Wavelength $=254 \mathrm{~nm}$

\begin{tabular}{|c|c|c|c|c|c|c|}
\hline $\begin{array}{c}\text { Peak } \\
\#\end{array}$ & $\begin{array}{c}\text { RetTime } \\
\text { [min] }\end{array}$ & Type & $\begin{array}{l}\text { Width } \\
\text { [min] }\end{array}$ & $\begin{array}{c}\text { Area } \\
{\left[\mathrm{mAU}^{*} \mathrm{~s}\right]}\end{array}$ & $\begin{array}{l}\text { Height } \\
{[\mathrm{mAU}]}\end{array}$ & $\begin{array}{c}\text { Area } \\
\%\end{array}$ \\
\hline . & & & & | $\ldots$ & & | \\
\hline 1 & 36. & BB & 1.0113 & 127.46680 & 1.85133 & 2.3146 \\
\hline 2 & 40.722 & BB & 1.1583 & 5379.60645 & 69.86516 & 97.6854 \\
\hline
\end{tabular}

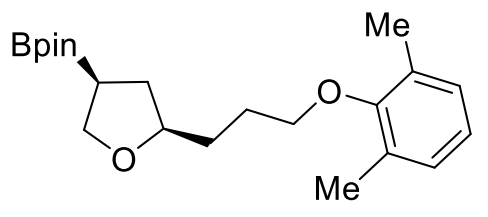

2-((3S,5R)-5-(3-(2,6-dimethylphenoxy)-propyl)-tetrahydrofuran-3-yl)-4,4,5,5-tetramethyl-1,3,2-dioxaborolane

(4be). This compound was synthesized according to general procedure B. The residue was purified by column chromatography on silica gel to afford the product 4 be $(99.0 \mathrm{mg}, 69 \%$ yield, $\mathrm{dr}=14: 1)$ as a colorless oil; ${ }^{1} \mathbf{H}$ NMR (400 MHz, Chloroform- $d$ ) $\delta 6.99(\mathrm{~d}, J=7.4 \mathrm{~Hz}, 2 \mathrm{H}), 6.90(\mathrm{dd}, J=8.2,6.7 \mathrm{~Hz}, 1 \mathrm{H}), 3.99(\mathrm{t}, J=8.4 \mathrm{~Hz}$, 1H), 3.89 (ddt, $J=9.0,6.3,5.5 \mathrm{~Hz}, 1 \mathrm{H}), 3.85-3.70$ (m, 3H), 2.27 (s, 6H), 2.18 (ddd, $J=11.9$, 
7.6, 5.5 Hz, 1H), 1.98 - $1.90(\mathrm{~m}, 1 \mathrm{H}), 1.88-1.67(\mathrm{~m}, 4 \mathrm{H}), 1.49(\mathrm{td}, J=11.8,9.2 \mathrm{~Hz}, 1 \mathrm{H}), 1.25$

(s, 12H) ppm; ${ }^{13} \mathbf{C}$ NMR (101 MHz, $\left.\mathbf{C D C l}_{3}\right) \delta 156.1,131.1,128.9,123.7,83.5,80.3,72.2$, 69.8, 35.2, 32.3, 27.6, 24.9, 16.4 ppm; ${ }^{11}$ B NMR (128 MHz, Chloroform-d) $\delta 32.39$ ppm; HRMS (ESI) calculated $[\mathrm{M}+\mathrm{H}]^{+}$for $\mathrm{C}_{21} \mathrm{H}_{34} \mathrm{BO}_{4}{ }^{+}=361.2545$, found: $361.2543 .[\boldsymbol{\alpha}]^{\mathbf{2 0}} \mathbf{D}=-2.9$ $\left(\mathrm{c}=0.15, \mathrm{CHCl}_{3}\right)$, the corresponding alcohol after oxidation. HPLC analysis (OD-H, $n$ Hexane: $i$-Propanol $=95: 5$ as eluent, $1 \mathrm{~mL} / \mathrm{min}, 220 \mathrm{~nm}$ ) indicated $95 \%$ ee: $\mathrm{t}_{\mathrm{R}}($ major $)=13.3$ $\min , t_{R}(\operatorname{minor})=15.3 \min$.
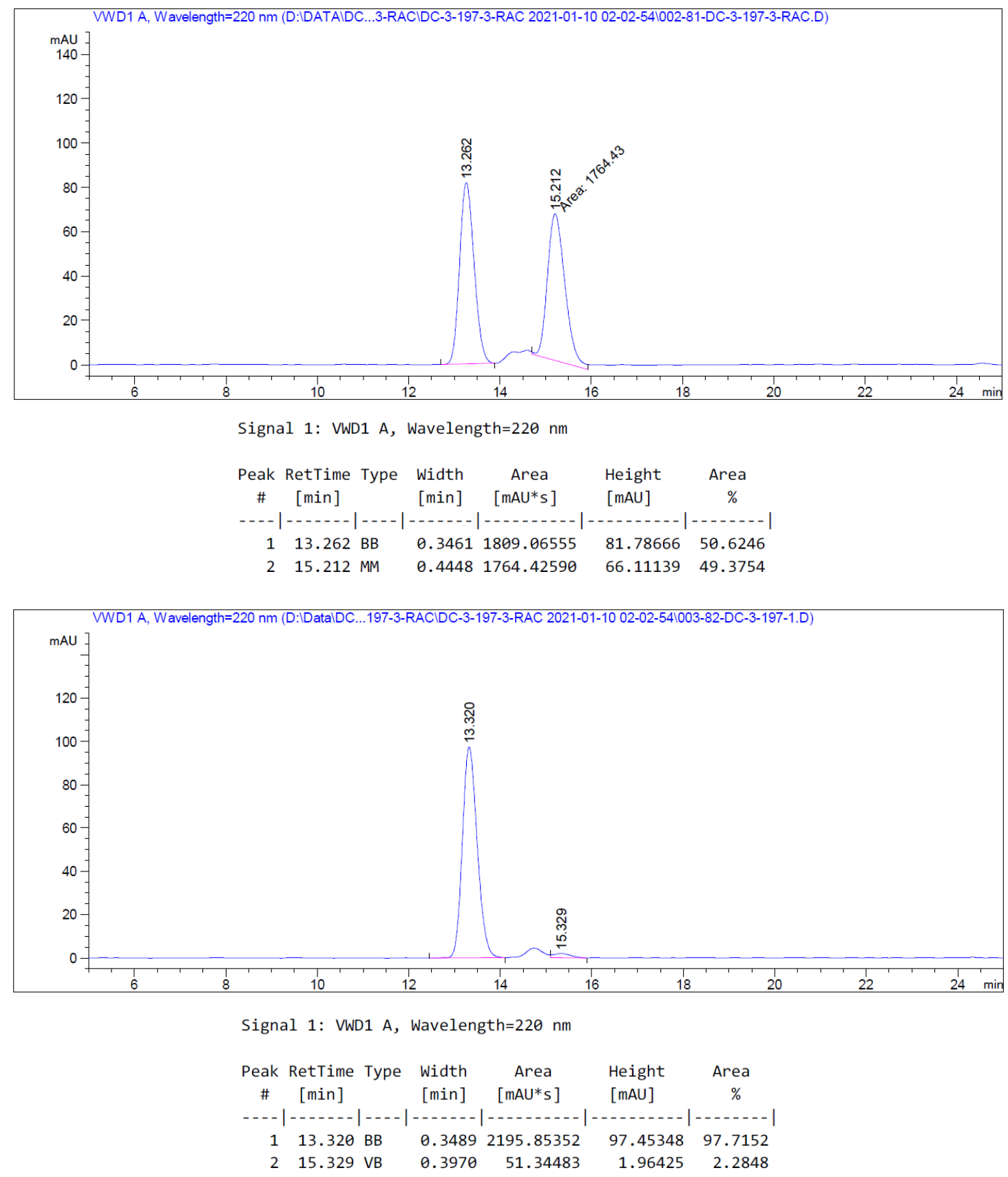


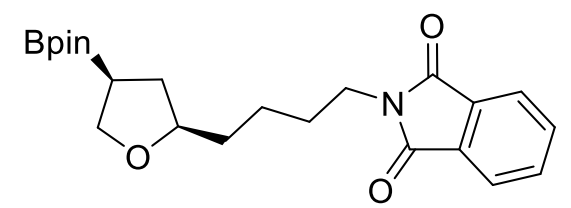

2-(4-((2R,4S)-4-(4,4,5,5-tetramethyl-1,3,2-dioxaborolan-2-yl)-tetrahydrofuran-2-yl)-butyl)-isoindoline-

1,3-dione (4bf). This compound was synthesized

according to general procedure B. The residue was purified by column chromatography on silica gel to afford the product $\mathbf{4 b f}(113.0 \mathrm{mg}, 71 \%$ yield, $\mathrm{dr}>20: 1)$ as a white solid; ${ }^{1} \mathbf{H}$ NMR (400 MHz, Chloroform-d) $\delta 7.78(\mathrm{dd}, J=5.5,3.0 \mathrm{~Hz}, 2 \mathrm{H}), 7.66(\mathrm{dd}, J=5.5,3.0 \mathrm{~Hz}, 2 \mathrm{H})$, $3.89(\mathrm{dd}, J=8.8,8.1 \mathrm{~Hz}, 1 \mathrm{H}), 3.78-3.66(\mathrm{~m}, 2 \mathrm{H}), 3.63(\mathrm{dd}, J=8.0,6.6 \mathrm{~Hz}, 2 \mathrm{H}), 2.07$ (ddd, $J$ $=12.0,7.7,5.4 \mathrm{~Hz}, 1 \mathrm{H}), 1.74-1.54(\mathrm{~m}, 4 \mathrm{H}), 1.50-1.39(\mathrm{~m}, 2 \mathrm{H}), 1.37-1.28(\mathrm{~m}, 2 \mathrm{H}), 1.19(\mathrm{~s}$, 12H) ppm; ${ }^{13} \mathbf{C}$ NMR (101 MHz, $\left.\mathbf{C D C l}_{3}\right) \delta$ 168.5, 133.9, 132.2, 123.2, 83.4, 80.2, 69.6, 38.0, 35.15, 35.08, 28.8, 24.8, 23.9 ppm; ${ }^{11}$ B NMR (128 MHz, Chloroform-d) $\delta 32.74$ ppm; HRMS (ESI) calculated $[\mathrm{M}+\mathrm{H}]^{+}$for $\mathrm{C}_{22} \mathrm{H}_{31} \mathrm{BNO}_{5}{ }^{+}=400.2290$, found: 400.2287 . $[\boldsymbol{\alpha}]^{\mathbf{2 0}} \mathbf{D}=-5.1(\mathrm{c}=$ $0.21, \mathrm{CHCl}_{3}$ ), the corresponding alcohol after oxidation. HPLC analysis (AS-H, $n$-Hexane: $i$ Propanol $=90: 10$ as eluent, $1 \mathrm{~mL} / \mathrm{min}, 220 \mathrm{~nm}$ ) indicated 93\% ee: $\mathrm{t}_{\mathrm{R}}($ minor $)=26.9 \mathrm{~min}$, $\mathrm{t}_{\mathrm{R}}($ major $)=35.7 \mathrm{~min}$.

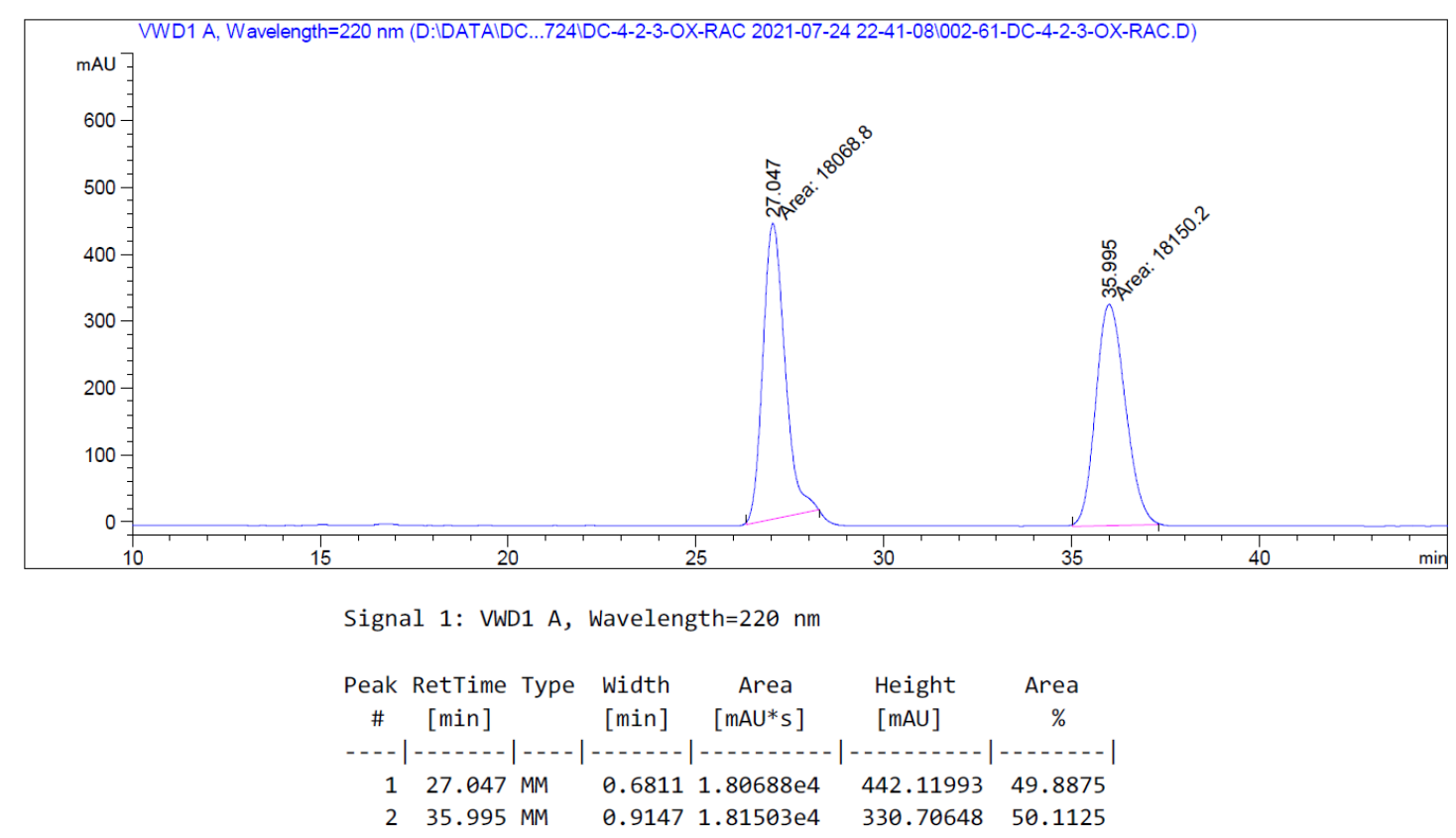



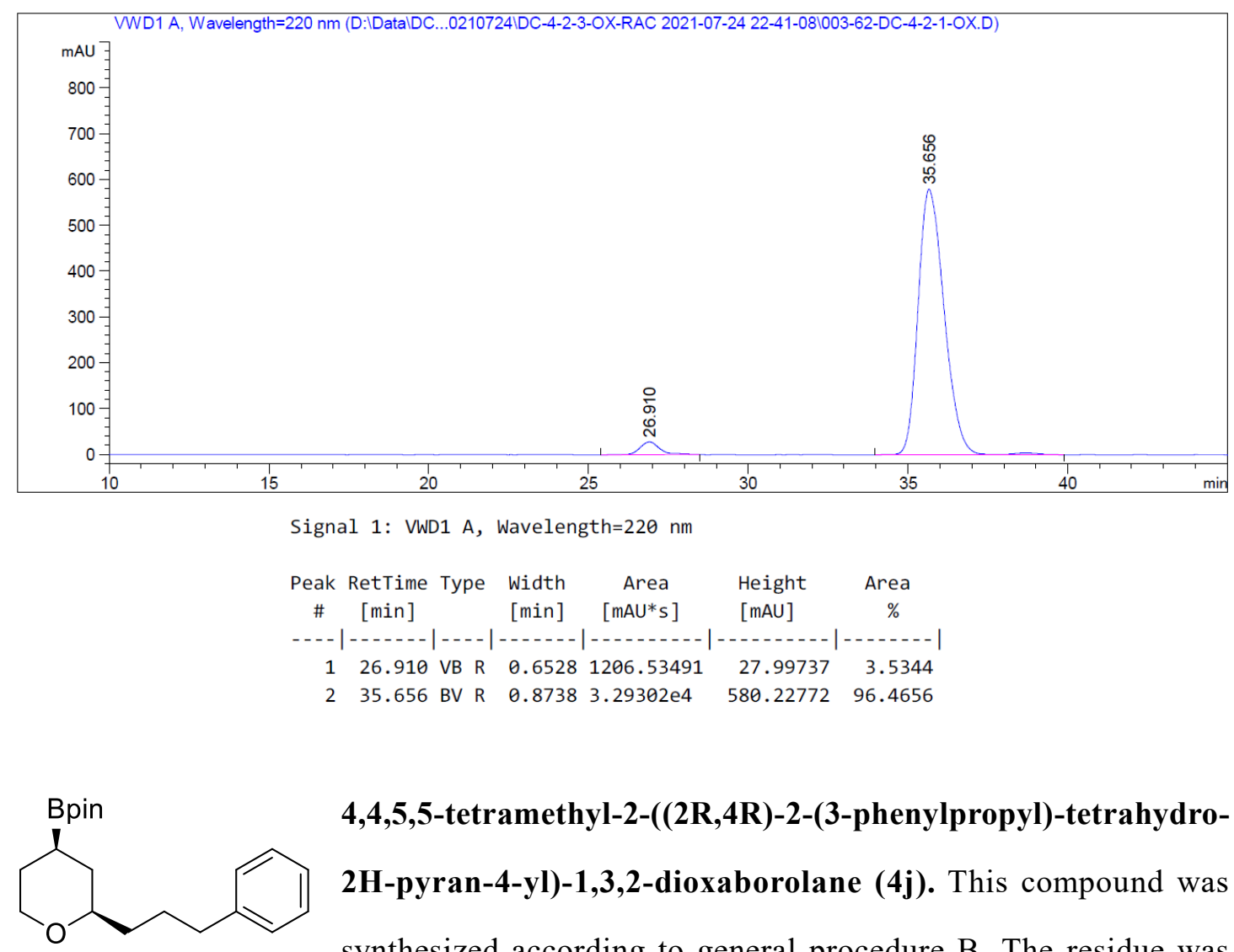

4,4,5,5-tetramethyl-2-((2R,4R)-2-(3-phenylpropyl)-tetrahydro2H-pyran-4-yl)-1,3,2-dioxaborolane $(\mathbf{4 j})$. This compound was synthesized according to general procedure B. The residue was purified by column chromatography on silica gel to afford the product $4 \mathbf{j}$ ( $26.1 \mathrm{mg}, 18 \%$ yield, $\mathrm{dr}>20: 1)$ as a colorless oil; ${ }^{1} \mathbf{H}$ NMR (600 MHz, Chloroform-d $) \delta 7.28$ - $7.23(\mathrm{~m}, 2 \mathrm{H}), 7.18$ $7.14(\mathrm{~m}, 3 \mathrm{H}), 3.98$ (ddd, $J=11.3,4.3,1.8 \mathrm{~Hz}, 1 \mathrm{H}), 3.39$ (td, $J=11.4,2.9 \mathrm{~Hz}, 1 \mathrm{H}), 3.22$ (dddd, $J=10.5,7.3,5.2,2.1 \mathrm{~Hz}, 1 \mathrm{H}), 2.60(\mathrm{t}, J=7.8 \mathrm{~Hz}, 2 \mathrm{H}), 1.79-1.70(\mathrm{~m}, 1 \mathrm{H}), 1.68-1.60(\mathrm{~m}$, 2H), $1.56-1.48(\mathrm{~m}, 3 \mathrm{H}), 1.46-1.39(\mathrm{~m}, 1 \mathrm{H}), 1.26-1.24(\mathrm{~m}, 1 \mathrm{H}), 1.23(\mathrm{~s}, 12 \mathrm{H}), 1.18-1.11$ (m, 1H). ${ }^{13} \mathbf{C}$ NMR (151 MHz, $\left.\mathbf{C D C l}_{3}\right) \delta$ 142.7, 128.6, 128.4, 125.7, 83.2, 78.3, 69.3, 36.4, 36.2, 33.0, 27.5, 27.4, 24.89, 24.86 ppm; ${ }^{11}$ B NMR (128 MHz, Chloroform-d) $\delta 32.64$ ppm; HRMS (ESI) calculated $[\mathrm{M}+\mathrm{H}]^{+}$for $\mathrm{C}_{20} \mathrm{H}_{32} \mathrm{BO}_{3}{ }^{+}=331.2439$, found: $331.2436 .[\boldsymbol{\alpha}]^{\mathbf{2 0}} \mathbf{D}=+3.0(\mathrm{c}$ $\left.=0.25, \mathrm{CHCl}_{3}\right)$, the corresponding alcohol after oxidation. HPLC analysis (OD-H, $n$-Hexane: $i$-Propanol $=95: 5$ as eluent, $1 \mathrm{~mL} / \mathrm{min}, 220 \mathrm{~nm}$ ) indicated $93 \%$ ee: $t_{\mathrm{R}}($ major $)=29.4 \mathrm{~min}$, $\mathrm{t}_{\mathrm{R}}(\operatorname{minor})=33.5 \mathrm{~min}$. 


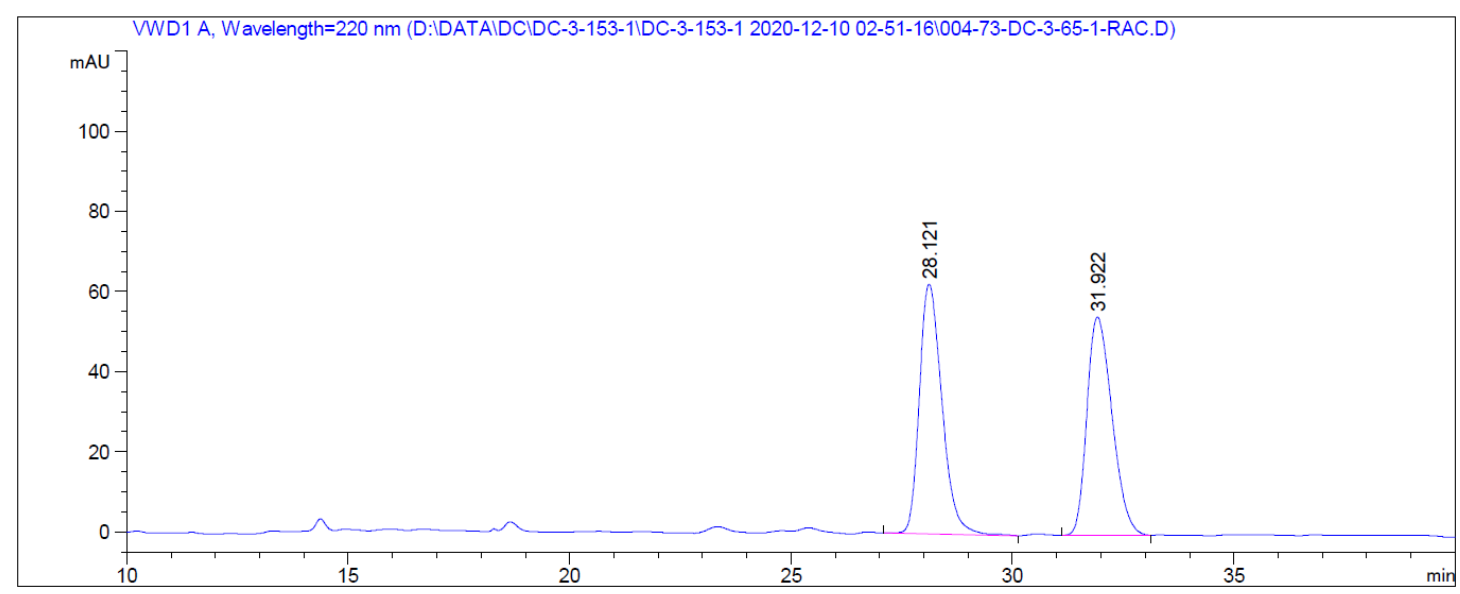

Signal 1: VWD1 A, Wavelength $=220 \mathrm{~nm}$
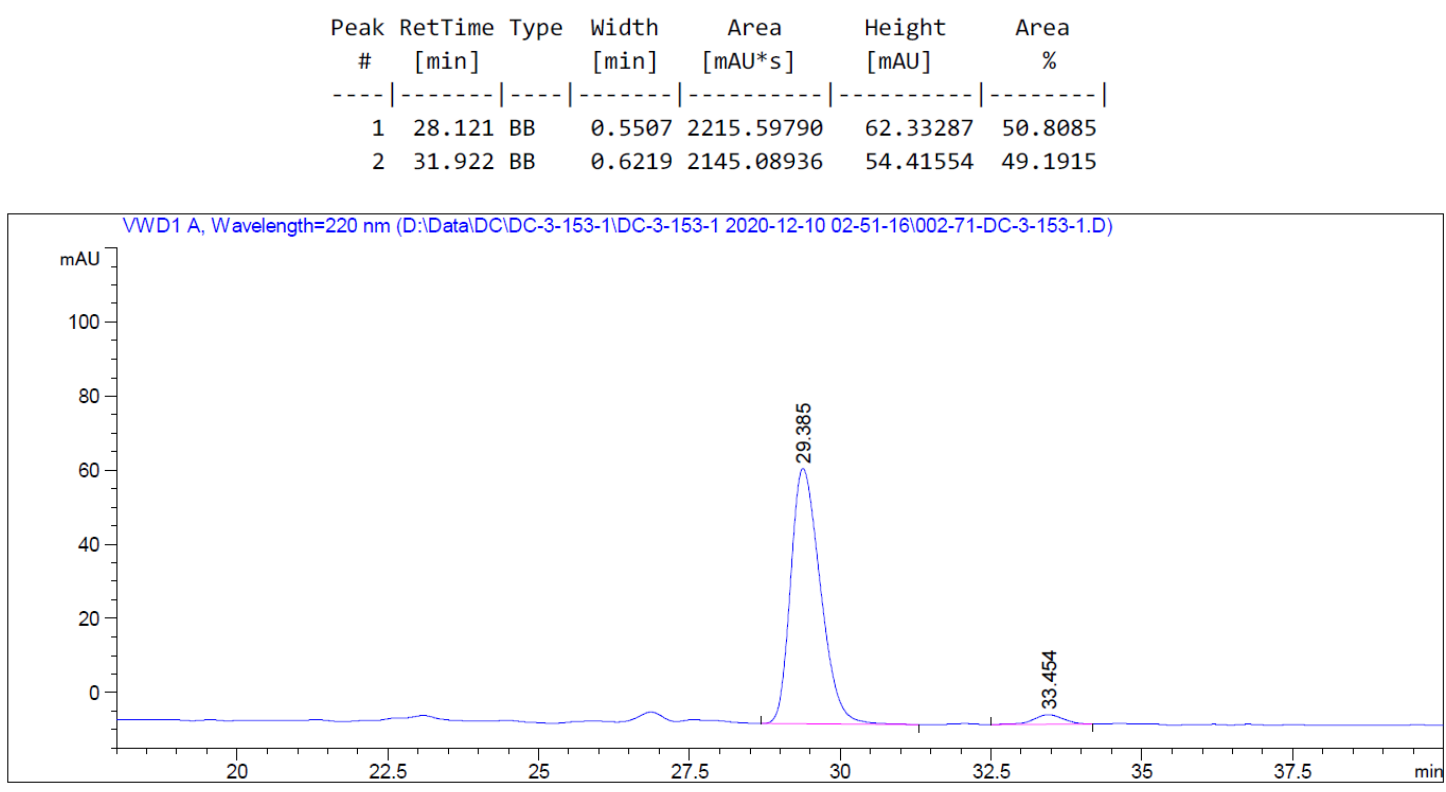

Signal 1: VWD1 A, Wavelength $=220 \mathrm{~nm}$
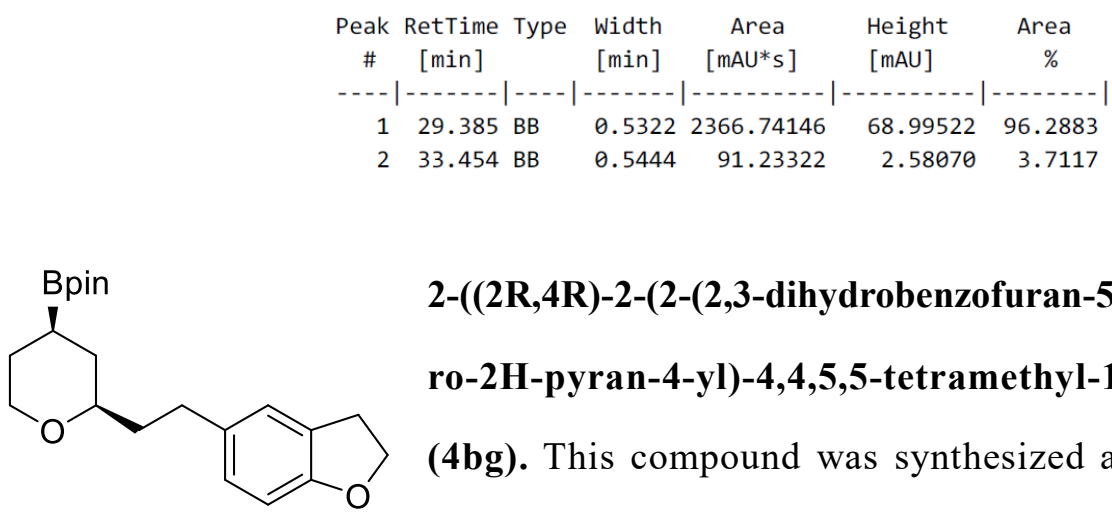

2-((2R,4R)-2-(2-(2,3-dihydrobenzofuran-5-yl)-ethyl)-tetrahydro-2H-pyran-4-yl)-4,4,5,5-tetramethyl-1,3,2-dioxaborolane

(4bg). This compound was synthesized according to general procedure B. The residue was purified by column chromatography on silica gel to afford the product $4 \mathbf{b g}(26.1 \mathrm{mg}, 18 \%$ yield, $\mathrm{dr}>20: 1)$ as a colorless oil; ${ }^{1} \mathbf{H}$ NMR (400 MHz, Chloroform-d) $\delta 7.00(\mathrm{~d}, J=1.9 \mathrm{~Hz}, 1 \mathrm{H}), 6.92-6.87(\mathrm{~m}, 1 \mathrm{H}), 6.67(\mathrm{~d}, J=$ $8.1 \mathrm{~Hz}, 1 \mathrm{H}), 4.52(\mathrm{t}, J=8.7 \mathrm{~Hz}, 2 \mathrm{H}), 4.04-3.97(\mathrm{~m}, 1 \mathrm{H}), 3.44-3.32(\mathrm{~m}, 1 \mathrm{H}), 3.24-3.10(\mathrm{~m}$, 
3H), 2.66 (ddd, $J=13.8,9.9,5.5 \mathrm{~Hz}, 1 \mathrm{H}), 2.56(\mathrm{ddd}, J=13.8,9.7,6.8 \mathrm{~Hz}, 1 \mathrm{H}), 1.74$ (dddd, $J$ $=13.4,9.7,7.7,5.6 \mathrm{~Hz}, 2 \mathrm{H}), 1.66-1.59(\mathrm{~m}, 2 \mathrm{H}), 1.57-1.45(\mathrm{~m}, 2 \mathrm{H}), 1.22(\mathrm{~s}, 12 \mathrm{H}), 1.18-$ 1.09 (m, 1H) ppm; ${ }^{13} \mathbf{C}$ NMR (101 MHz, $\left.\mathbf{C D C l}_{3}\right) \delta$ 158.2, 134.5, 127.9, 127.0, 125.0, 109.0, 83.2, 77.6, 71.2, 69.3, 38.9, 32.9, 31.2, 29.9, 27.4, 24.85, 24.83 ppm; ${ }^{11}$ B NMR (128 MHz, Chloroform-d) $\delta 33.40$ ppm; HRMS (ESI) calculated $[\mathrm{M}+\mathrm{H}]^{+}$for $\mathrm{C}_{21} \mathrm{H}_{32} \mathrm{BO}_{4}{ }^{+}=359.2388$, found: $359.2381 .[\boldsymbol{\alpha}]^{20}{ }_{\mathbf{D}}=+6.0\left(\mathrm{c}=0.25, \mathrm{CHCl}_{3}\right)$, the corresponding alcohol after oxidation. HPLC analysis (OD-H, $n$-Hexane: $i$-Propanol $=90: 10$ as eluent, $1 \mathrm{~mL} / \mathrm{min}, 254 \mathrm{~nm}$ ) indicated $93 \%$ ee: $t_{R}($ minor $)=21.0 \mathrm{~min}, t_{R}($ major $)=27.1 \mathrm{~min}$.
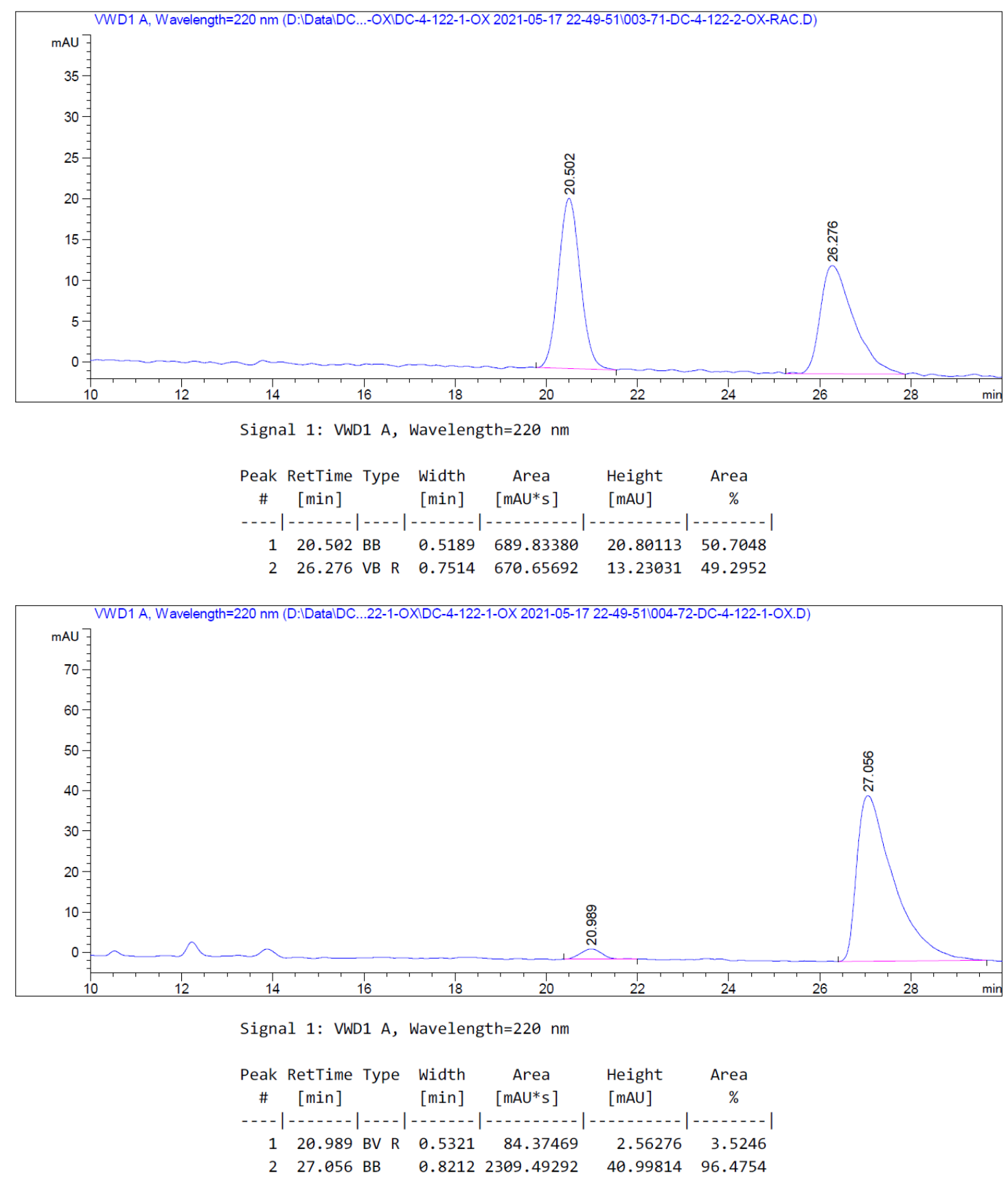


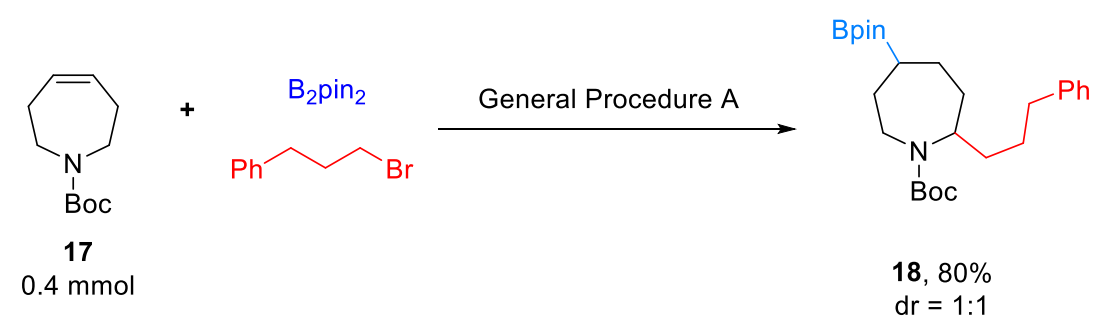

Following the General Procedure A using 17, the crude product was purified by flash column chromatography on silica gel to afford $\mathbf{1 8}$ as a colorless oil $(141.6 \mathrm{mg}, 80 \%, \mathrm{dr}=1: 1)$.

tert-butyl 2-(3-phenylpropyl)-5-(4,4,5,5-tetramethyl-1,3,2-dioxaborolan-2-yl)-azepane-1carboxylate (18). The mixture ${ }^{1} \mathbf{H}$ NMR (400 MHz, Chloroform-d) $\delta 7.31-7.21$ (m, 4H), 7.18 - $7.11(\mathrm{~m}, 6 \mathrm{H}), 4.08(\mathrm{dq}, J=10.4,6.8 \mathrm{~Hz}, 1 \mathrm{H}), 3.86(\mathrm{ddd}, J=13.8,11.4,6.3 \mathrm{~Hz}, 1 \mathrm{H}), 3.66$ $(\mathrm{dt}, J=14.5,3.9 \mathrm{~Hz}, 1 \mathrm{H}), 3.55(\mathrm{dt}, J=14.7,3.5 \mathrm{~Hz}, 1 \mathrm{H}), 2.83-2.68(\mathrm{~m}, 2 \mathrm{H}), 2.64-2.55(\mathrm{~m}$, 4H), 2.02 - $1.71(\mathrm{~m}, 8 \mathrm{H}), 1.67$ - $1.54(\mathrm{~m}, 6 \mathrm{H}), 1.44(\mathrm{~s}, 9 \mathrm{H}), 1.41(\mathrm{~s}, 9 \mathrm{H}), 1.40$ - $1.37(\mathrm{~m}, 2 \mathrm{H}), 1.36$ - $1.29(\mathrm{~m}, 4 \mathrm{H}), 1.22(\mathrm{~d}, \mathrm{~J}=1.5 \mathrm{~Hz}, 24 \mathrm{H}), 1.20-1.13(\mathrm{~m}, 2 \mathrm{H})$. The mixture ${ }^{13} \mathbf{C}$ NMR $(\mathbf{1 0 1}$ MHz, $\left.\mathbf{C D C l}_{3}\right) \delta 156.2,155.9,142.8,142.5,128.5,128.4,128.43,128.27,125.8,125.6,83.2$, 79.0, 78.7, 55.3, 53.9, 41.6, 35.9, 35.8, 34.5, 34.3, 33.8, 33.5, 30.0, 29.5, 28.7, 28.1, 28.0, 26.2, 25.85, 24.93, 24.90, 22.4 ppm; ${ }^{11}$ B NMR (128 MHz, Chloroform-d) $\delta 35.51$ ppm; HRMS (ESI) calculated $[\mathrm{M}+\mathrm{H}]^{+}$for $\mathrm{C}_{26} \mathrm{H}_{43} \mathrm{BNO}_{4}{ }^{+}=444.3280$, found: 444.3273 .

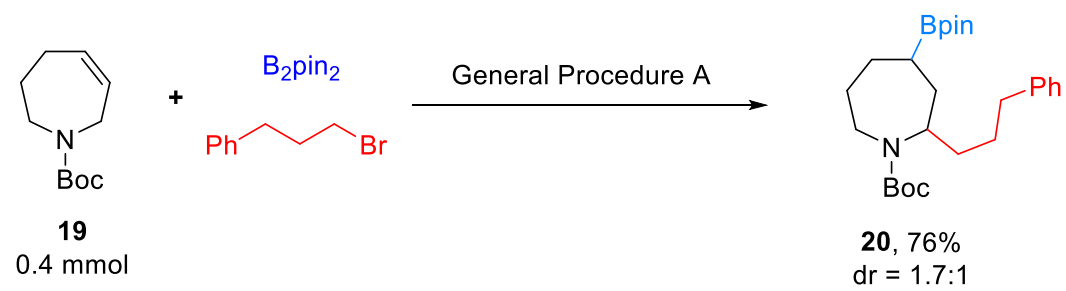

Following the General Procedure A using 19, the crude product was purified by flash column chromatography on silica gel to afford $\mathbf{2 0}$ as a colorless oil $(134.1 \mathrm{mg}, 76 \%, \mathrm{dr}=1.7: 1)$.

tert-butyl 2-(3-phenylpropyl)-4-(4,4,5,5-tetramethyl-1,3,2-dioxaborolan-2-yl)-azepane-1carboxylate (20). Major isomer ${ }^{1} \mathbf{H}$ NMR (600 MHz, Chloroform-d) $\delta 7.28$ - 7.23 (m, 2H), $7.19-7.13(\mathrm{~m}, 3 \mathrm{H}), 4.11(\mathrm{dq}, \mathrm{J}=11.5,6.9 \mathrm{~Hz}, 1 \mathrm{H}), 3.69-3.65(\mathrm{~m}, 1 \mathrm{H}), 2.68-2.54(\mathrm{~m}, 3 \mathrm{H})$, $2.04-1.96(\mathrm{~m}, 1 \mathrm{H}), 1.88(\mathrm{~m}, 1 \mathrm{H}), 1.77(\mathrm{~m}, 1 \mathrm{H}), 1.64-1.56(\mathrm{~m}, 2 \mathrm{H}), 1.46(\mathrm{~s}, 9 \mathrm{H}), 1.40-1.32$ $(\mathrm{m}, 3 \mathrm{H}), 1.21(\mathrm{~d}, \mathrm{~J}=4.2 \mathrm{~Hz}, 12 \mathrm{H}), 1.20-1.10(\mathrm{~m}, 3 \mathrm{H})$; Minor isomer ${ }^{1} \mathbf{H}$ NMR (600 MHz, Chloroform-d) $\delta 7.28-7.23(\mathrm{~m}, 2 \mathrm{H}), 7.19-7.13(\mathrm{~m}, 3 \mathrm{H}), 3.97-3.88(\mathrm{~m}, 1 \mathrm{H}), 3.80(\mathrm{~d}, \mathrm{~J}=$ $14.3 \mathrm{~Hz}, 1 \mathrm{H}), 2.68-2.54(\mathrm{~m}, 3 \mathrm{H}), 2.04-1.96(\mathrm{~m}, 1 \mathrm{H}), 1.88(\mathrm{~m}, 1 \mathrm{H}), 1.77(\mathrm{~m}, 1 \mathrm{H}), 1.64-1.56$ 
(m, 2H), $1.42(\mathrm{~s}, 9 \mathrm{H}), 1.40$ - $1.32(\mathrm{~m}, 3 \mathrm{H}), 1.23(\mathrm{~s}, \mathrm{~J}=4.2 \mathrm{~Hz}, 12 \mathrm{H}), 1.20$ - $1.10(\mathrm{~m}, 3 \mathrm{H}) \mathrm{ppm}$;

The mixture ${ }^{13} \mathbf{C}$ NMR (101 MHz, $\left.\mathbf{C D C l}_{3}\right) \delta 156.1,155.8,142.8,142.5,128.5,128.4,128.34$, $128.27,125.7,125.6,83.1,83.08,79.0,78.7,55.1,54.0,42.8,42.3,36.0,35.9,35.0,34.8,34.7$, 34.6, 31.9, 31.7, 28.6, 28.56, 28.1, 28.0, 26.5, 26.4, 24.9, 24.8, 24.6 ppm; ${ }^{11} \mathbf{B}$ NMR (128 MHz, Chloroform-d) $\delta 34.49$ ppm; HRMS (ESI) calculated $[\mathrm{M}+\mathrm{H}]^{+}$for $\mathrm{C}_{26} \mathrm{H}_{43} \mathrm{BNO}_{4}{ }^{+}=444.3280$, found: 444.3272 .

\section{Scale-up Experiment and Transformations of Products}

\subsection{Scale-up Experiment}
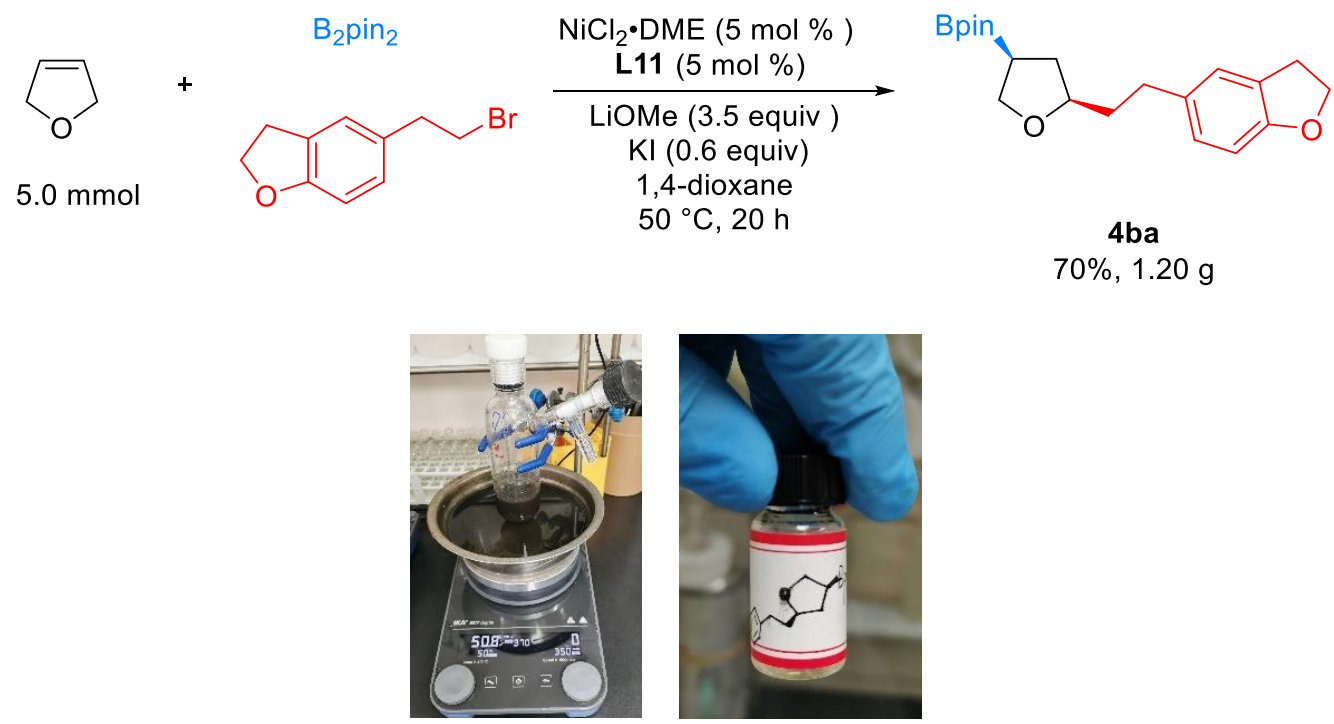

Fig S1. The Scale-up Reaction

In a glove box, to an oven-dried $120 \mathrm{~mL}$ reaction tube which equipped with a magnetic stir bar and sealed with a rubber stopper sequentially was added $\mathrm{NiCl}_{2} \cdot \mathrm{DME}(54.9 \mathrm{mg}, 0.25 \mathrm{mmol}, 5$ mol \%), L11 (64.7mg, 0.25 mmol, 5 mol \%), LiOMe (665.0 mg, 17.5 mmol, 3.5 equiv), KI (500.0 mg, $3.0 \mathrm{mmol}, 0.6$ equiv) and bis(pinacolato)diboron ( $4.4 \mathrm{~g}, 17.5 \mathrm{mmol}, 3.5$ equiv). Then anhydrous 1,4-dioxane ( $12.5 \mathrm{~mL})$, alkene (5.0 mmol, 1.0 equiv), alkylbromide ( $7.5 \mathrm{mmol}$, 1.5 equiv), anhydrous dioxane ( $12.5 \mathrm{~mL})$ were added and the mixture was stirred. After $20 \mathrm{~h}$ of stirring at $50{ }^{\circ} \mathrm{C}$, the resulting mixture was quenched with water $(30 \mathrm{~mL})$ and further diluted with ethyl acetate $(50 \mathrm{~mL})$. Then the mixture was extracted with ethyl acetate and the combined 
organic layers were dried over anhydrous $\mathrm{Na}_{2} \mathrm{SO}_{4}$, filtered and concentrated under vacuum. The crude material was separated on a silica gel column affording the desired product as a colorless oil (1.20 g, 70\% yield).

\subsection{Transformations of Products}

5-(2-((2R,4R)-4-vinyltetrahydrofuran-2-yl)-ethyl)-2,3-dihydrobenzofuran ${ }^{4}$
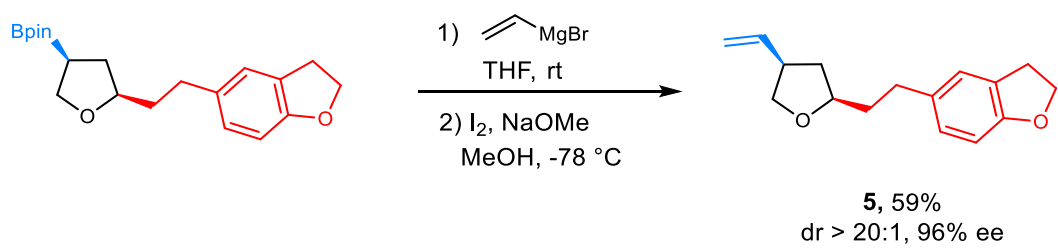

To an oven-dried $10 \mathrm{~mL}$ reaction tube containing a stirring bar, a solution of compound 4ba (68.9 mg, $0.2 \mathrm{mmol}, 1.0$ equiv) in THF ( $2 \mathrm{~mL})$ was added as subsequently vinylmagnesium bromide $(0.8 \mathrm{~mL}, 1 \mathrm{M}, 0.8 \mathrm{mmol}, 4$ equiv) was added dropwise and stirred at room temperature for $30 \mathrm{~min}$. To the above solution at $-78^{\circ} \mathrm{C}, \mathrm{I}_{2}(203 \mathrm{mg}, 0.8$ mmol, 4.0 equiv) in methanol $(3.0 \mathrm{~mL})$ was added dropwise. The reaction mixture was allowed to stir $30 \mathrm{~min}$ at the same temperature followed by dropwise addition of a solution of $\mathrm{NaOMe}(87 \mathrm{mg}, 1.6 \mathrm{mmol}, 8$ equiv) in methanol (3 mL). After warming to room temperature, the mixture was stirred for another $1.5 \mathrm{~h}$, diluted with pentane (20 $\mathrm{mL}$ ) and washed sequentially with saturated aqueous solution of $\mathrm{Na}_{2} \mathrm{~S}_{2} \mathrm{O}_{3}(5 \mathrm{~mL})$. The mixture was extracted with EtOAc $(3 \times 10 \mathrm{~mL})$, dried over $\mathrm{Na}_{2} \mathrm{SO}_{4}$, filtered and concentrated under reduced pressure. The mixture was purified by flash column chromatography to provide the title compound $\mathbf{5}$ as a colorless oil (29.0 mg, 59\% yield, dr $>20: 1,96 \%$ ee).

${ }^{1}$ H NMR $(400 \mathrm{MHz}$, Chloroform- $d) \delta 7.04(\mathrm{~d}, J=1.8 \mathrm{~Hz}, 1 \mathrm{H}), 6.95-6.90(\mathrm{~m}, 1 \mathrm{H}), 6.70(\mathrm{~d}, J$ $=8.1 \mathrm{~Hz}, 1 \mathrm{H}), 5.75(\mathrm{ddd}, J=17.1,10.2,8.1 \mathrm{~Hz}, 1 \mathrm{H}), 5.14-4.89(\mathrm{~m}, 2 \mathrm{H}), 4.54(\mathrm{t}, J=8.7 \mathrm{~Hz}$, 2H), $3.97-3.83(\mathrm{~m}, 2 \mathrm{H}), 3.56(\mathrm{t}, J=8.2 \mathrm{~Hz}, 1 \mathrm{H}), 3.28-3.03(\mathrm{~m}, 2 \mathrm{H}), 3.00-2.81(\mathrm{~m}, 1 \mathrm{H}), 2.68$ (ddd, $J=13.8,10.0,5.8 \mathrm{~Hz}, 1 \mathrm{H}), 2.58(\mathrm{ddd}, J=13.8,9.7,6.4 \mathrm{~Hz}, 1 \mathrm{H}), 2.17$ (ddd, $J=12.2,7.5$, $5.6 \mathrm{~Hz}, 1 \mathrm{H}), 1.89$ (dddd, $J=13.3,9.8,7.5,5.8 \mathrm{~Hz}, 1 \mathrm{H}), 1.76$ (dddd, $J=13.4,10.0,6.5,5.4 \mathrm{~Hz}$, $1 \mathrm{H}), 1.36(\mathrm{dt}, J=12.2,9.6 \mathrm{~Hz}, 1 \mathrm{H}) \mathrm{ppm} ;{ }^{13} \mathbf{C} \mathbf{N M R}\left(101 \mathrm{MHz}, \mathrm{CDCl}_{3}\right) \delta 158.3,139.4,134.1$, 
127.9, 127.1, 125.0, 115.2, 109.1, 79.5, 72.3, 71.3, 44.6, 39.3, 38.2, 32.2, 29.9 ppm; HRMS

(ESI) calculated $[\mathrm{M}+\mathrm{H}]^{+}$for $\mathrm{C}_{16} \mathrm{H}_{21} \mathrm{O}_{2}{ }^{+}=245.1536$, found: 245.1530. $[\boldsymbol{\alpha}]^{20} \mathbf{D}=+8.8(\mathrm{c}=0.16$, $\mathrm{CHCl}_{3}$ ). HPLC analysis (OD-H, $n$-Hexane: $i$-Propanol $=95: 5$ as eluent, $1 \mathrm{~mL} / \mathrm{min}, 220 \mathrm{~nm}$ ) indicated $96 \%$ ee: $t_{R}($ minor $)=5.2 \mathrm{~min}, \mathrm{t}_{\mathrm{R}}($ major $)=5.5 \mathrm{~min}$

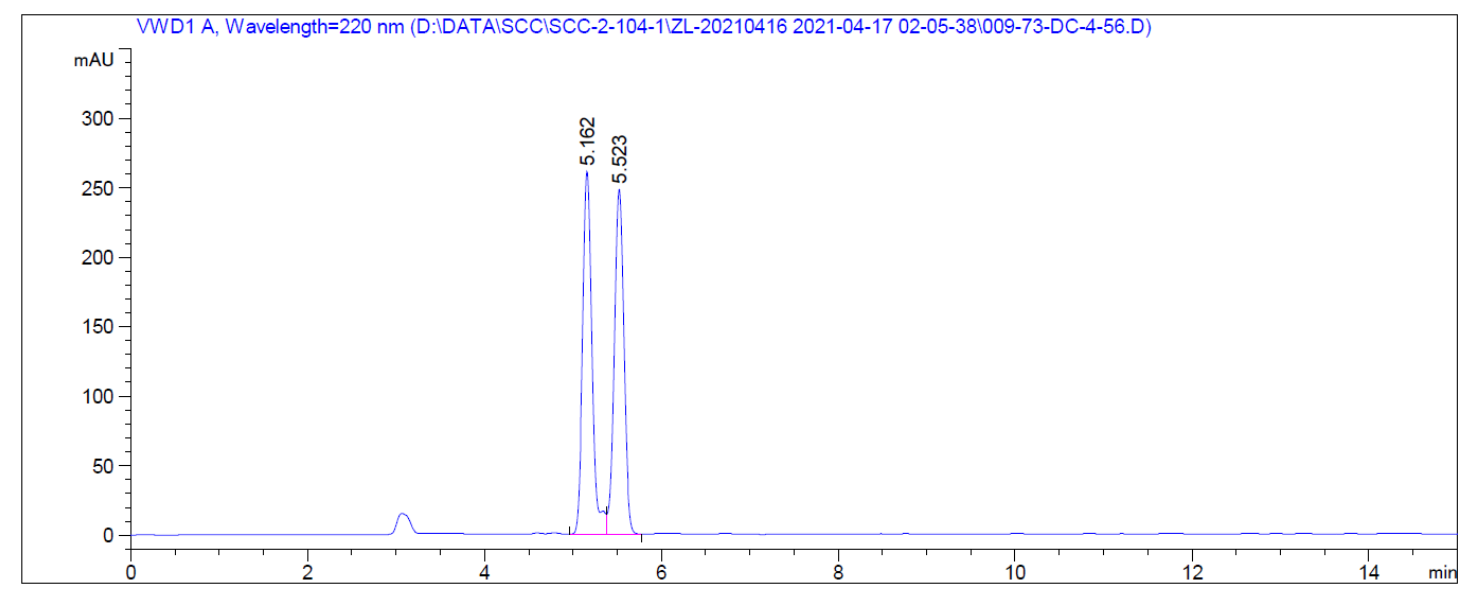

Signal 1: VWD1 A, Wavelength $=220 \mathrm{~nm}$
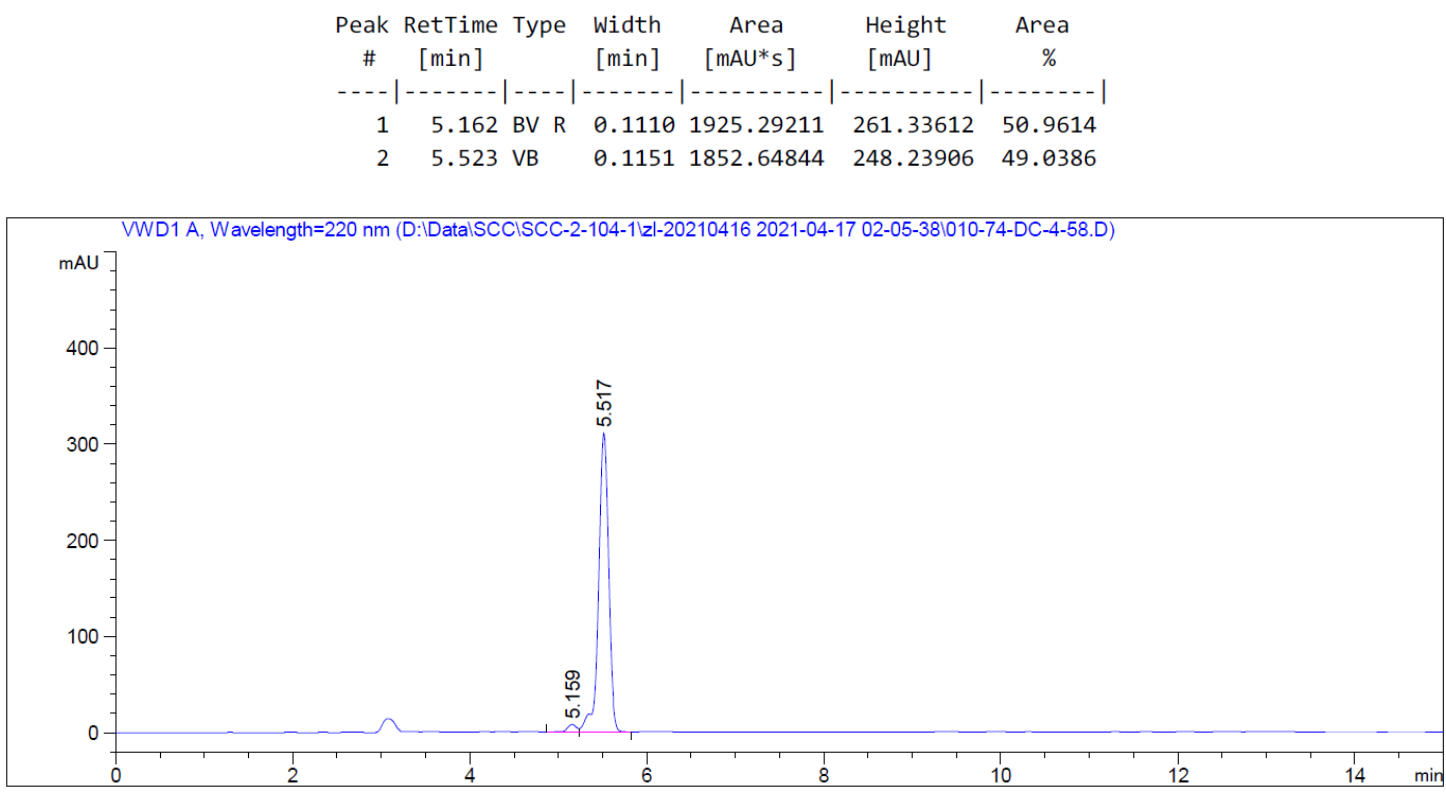

Signal 1: VWD1 A, Wavelength $=220 \mathrm{~nm}$

\begin{tabular}{|c|c|c|c|c|c|c|}
\hline $\begin{array}{c}\text { eak } \\
\text { \# }\end{array}$ & $\begin{array}{c}\text { RetTime } \\
\text { [min] }\end{array}$ & Type & $\begin{array}{l}\text { Width } \\
\text { [min] }\end{array}$ & $\begin{array}{c}\text { Area } \\
{\left[\mathrm{mAU}^{*} \mathrm{~s}\right]}\end{array}$ & $\begin{array}{l}\text { Height } \\
{[\mathrm{mAU}]}\end{array}$ & $\begin{array}{c}\text { Area } \\
\%\end{array}$ \\
\hline & & & & & & \\
\hline 1 & & & 0. & 54 & 7. & 308 \\
\hline 2 & 5.517 & B & & 2428.75391 & 7571 & 97.8192 \\
\hline
\end{tabular}

2-((3R,5R)-5-(2-(2,3-dihydrobenzofuran-5-yl)-ethyl)-tetrahydrofuran-3-yl)-isoindoline-

\section{1,3-dione ${ }^{5}$}



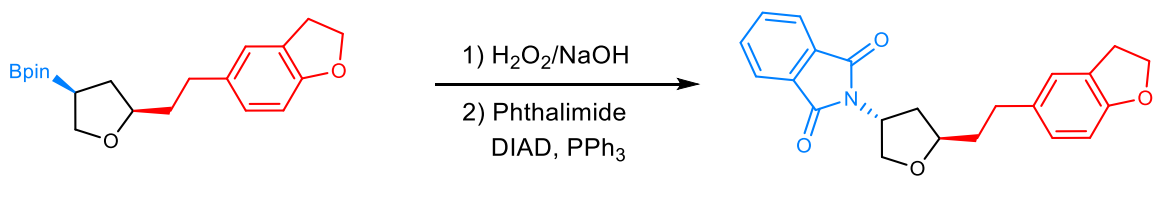

$$
\begin{gathered}
\mathbf{6}, 81 \% \\
d r>20: 1,95 \% \text { ee }
\end{gathered}
$$

In a $50 \mathrm{~mL}$ round bottom flask, a solution of compound $\mathbf{4 b a}(68.9 \mathrm{mg}, 0.2 \mathrm{mmol}, 1.0$ equiv) was dissolved in THF $(2.0 \mathrm{~mL})$. The reaction flask was placed in an ice bath and cooled to 0 ${ }^{\circ} \mathrm{C}$, then $\mathrm{NaOH}\left(1.2 \mathrm{~mL}, 2 \mathrm{M}\right.$ aqueous solution, $2 \mathrm{mmol}, 10.0$ equiv) and $\mathrm{H}_{2} \mathrm{O}_{2}(1.2 \mathrm{~mL}, 30 \%$ aqueous solution, $1.1 \mathrm{mmol}, 6.0$ equiv) were added. The ice bath was removed and the solution was stirred at room temperature for 4 hours. Then the reaction was quenched with saturated aqueous solution of $\mathrm{Na}_{2} \mathrm{~S}_{2} \mathrm{O}_{3}(5 \mathrm{~mL})$ under an ice bath. The aqueous layer was extracted with EtOAc $(3 \times 5 \mathrm{~mL})$. The combined organic phases were dried over anhydrous $\mathrm{Na}_{2} \mathrm{SO}_{4}$ and the solvent was removed under reduced pressure. The crude material, phthalimide ( $44.0 \mathrm{mg}, 0.3$ mmol, 1.5 equiv), and $\mathrm{PPh}_{3}(78.7 \mathrm{mg}, 0.3 \mathrm{mmol}, 1.5$ equiv) were dissolved in anhydrous THF $(2.0 \mathrm{~mL})$ at $0{ }^{\circ} \mathrm{C}$. To this mixture was added dropwise DIAD $(61.0 \mathrm{mg}, 0.3 \mathrm{mmol}, 1.5$ equiv) over a period of $5 \mathrm{~min}$, the reaction was warmed to $\mathrm{rt}$ and stirred $2 \mathrm{~h}$. Then the reaction was quenched with saturated aqueous solution of $\mathrm{NaCl}(5 \mathrm{~mL})$ and extracted with EtOAc $(3 \times 5$ $\mathrm{mL}$ ), dried over $\mathrm{Na}_{2} \mathrm{SO}_{4}$, filtered and concentrated under reduced pressure. The mixture was purified by flash column chromatography to provide the title compound $\mathbf{6}$ as white solid (58.7 mg, $81 \%$ yield, $\mathrm{dr}>20: 1,95 \%$ ee).

${ }^{1}$ H NMR (400 MHz, Chloroform-d) $\delta 7.84(\mathrm{dd}, J=5.4,3.0 \mathrm{~Hz}, 2 \mathrm{H}), 7.73(\mathrm{dd}, J=5.5,3.0 \mathrm{~Hz}$, 2H), $7.06(\mathrm{~d}, J=1.9 \mathrm{~Hz}, 1 \mathrm{H}), 6.95(\mathrm{dd}, J=8.1,1.9 \mathrm{~Hz}, 1 \mathrm{H}), 6.71(\mathrm{~d}, J=8.1 \mathrm{~Hz}, 1 \mathrm{H}), 4.94(\mathrm{dtd}$, $J=10.8,7.9,4.6 \mathrm{~Hz}, 1 \mathrm{H}), 4.55(\mathrm{t}, J=8.7 \mathrm{~Hz}, 2 \mathrm{H}), 4.47-4.36(\mathrm{~m}, 1 \mathrm{H}), 4.17(\mathrm{t}, J=8.4 \mathrm{~Hz}, 1 \mathrm{H})$, $3.93(\mathrm{dd}, J=8.8,7.7 \mathrm{~Hz}, 1 \mathrm{H}), 3.19(\mathrm{t}, J=8.6 \mathrm{~Hz}, 2 \mathrm{H}), 2.75(\mathrm{ddd}, J=13.7,10.3,5.5 \mathrm{~Hz}, 1 \mathrm{H})$, $2.63(\mathrm{ddd}, J=13.8,10.1,6.3 \mathrm{~Hz}, 1 \mathrm{H}), 2.42(\mathrm{ddd}, J=12.9,6.7,4.6 \mathrm{~Hz}, 1 \mathrm{H}), 1.99$ - $1.78(\mathrm{~m}$, 3H). ${ }^{13} \mathbf{C}$ NMR (101 MHz, $\left.\mathbf{C D C l}_{3}\right) \delta 168.2,158.4,134.3,134.1,131.9,127.8,127.1,125.0$, 123.4, 109.1, 79.4, 71.3, 69.3, 49.4, 37.8, 35.7, 32.0, 29.9 ppm; HRMS (ESI) calculated $[\mathrm{M}+\mathrm{H}]^{+}$for $\mathrm{C}_{22} \mathrm{H}_{22} \mathrm{NO}_{4}{ }^{+}=364.1543$, found: $364.1539 .[\boldsymbol{\alpha}]^{20}{ }_{\mathrm{D}}=+34.1\left(\mathrm{c}=0.36, \mathrm{CHCl}_{3}\right) . \mathbf{H P L C}$ analysis (AD-H, $n$-Hexane $: i$-Propanol $=95: 5$ as eluent, $1 \mathrm{~mL} / \mathrm{min}, 220 \mathrm{~nm}$ ) indicated $95 \%$ ee: $t_{R}($ major $)=29.5 \mathrm{~min}, t_{R}($ minor $)=36.9 \min$. 


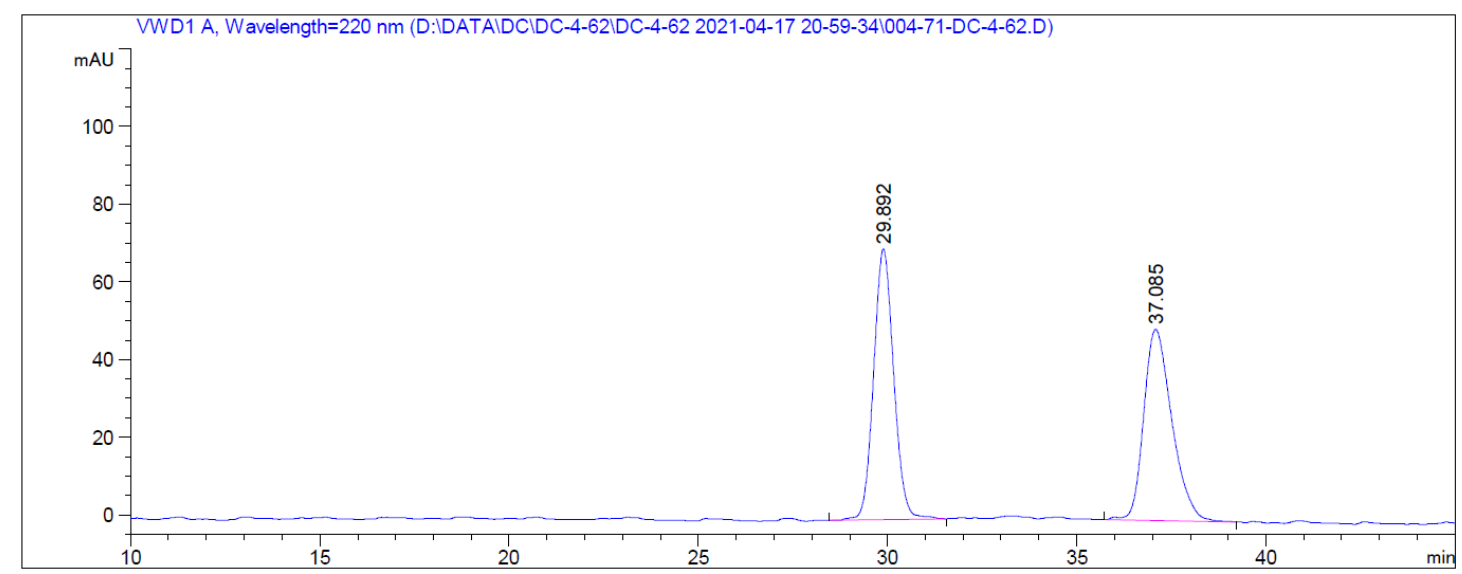

Signal 1: VWD1 A, Wavelength $=220 \mathrm{~nm}$
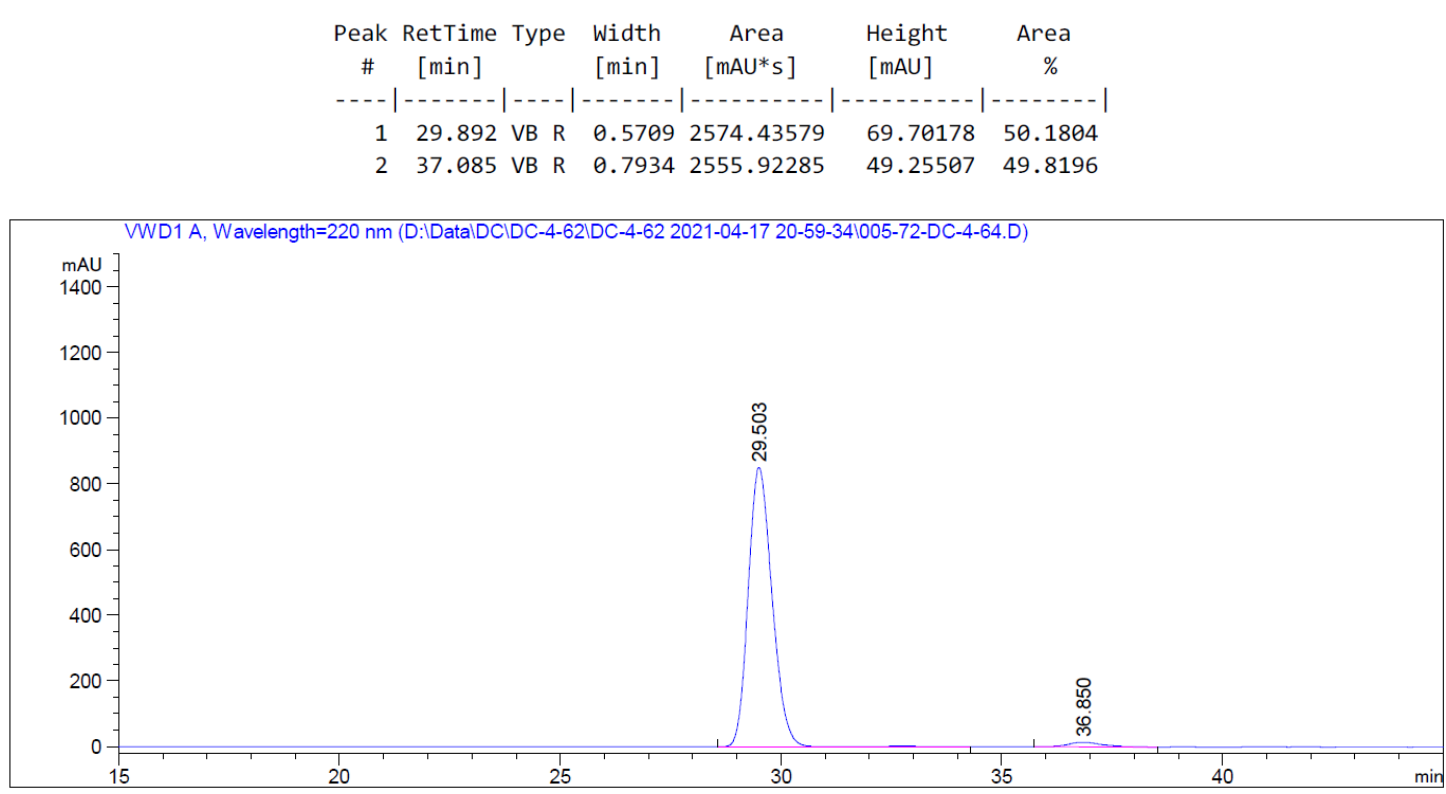

Signal 1: VWD1 A, Wavelength $=220 \mathrm{~nm}$

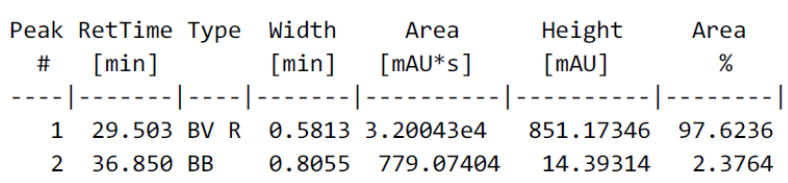

\section{5-(2-((2R,4S)-4-(furan-2-yl)-tetrahydrofuran-2-yl)-ethyl)-2,3-dihydrobenzofuran ${ }^{6}$}
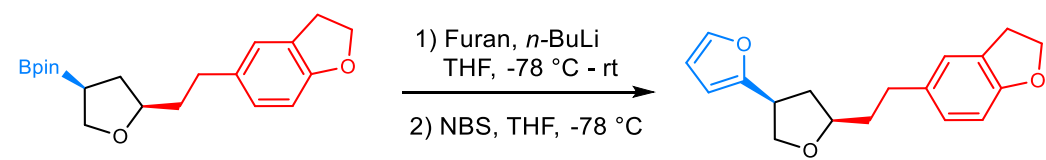

$7,57 \%$

$\mathrm{dr}>20: 1,92 \%$ ee

To an oven-dried $10 \mathrm{~mL}$ reaction tube containing a stirring bar, a solution of furan $(22 \mu \mathrm{L}$, $0.3 \mathrm{mmol}, 1.5$ equiv $)$ in THF $(1.0 \mathrm{~mL})$ was cooled to $-78{ }^{\circ} \mathrm{C}$. Then $n$-BuLi $(0.12 \mathrm{~mL}, 0.3 \mathrm{mmol}$, 2.5 $\mathrm{M}$ in hexane) was added dropwise under $-78^{\circ} \mathrm{C}$. The mixture was warmed to room 
temperature and stirred for $2 \mathrm{~h}$. Subsequently, the reaction mixture was cooled back to $-78{ }^{\circ} \mathrm{C}$ and a solution of compound $\mathbf{4 b a}(68.9 \mathrm{mg}, 0.2 \mathrm{mmol}, 1.0$ equiv) in THF ( $0.5 \mathrm{ml})$ was added dropwise. The resulting mixture was stirred at the same temperature for $1 \mathrm{~h}$. Then a solution of NBS (44.0 mg, $0.24 \mathrm{mmol}, 1.2$ equiv) in THF (1.0 mL) was added dropwise and stirred $1 \mathrm{~h}$ at $-78^{\circ} \mathrm{C}$. Saturated aqueous $\mathrm{Na}_{2} \mathrm{~S}_{2} \mathrm{O}_{3}$ solution $(2.0 \mathrm{~mL})$ was added and the reaction mixture was allowed to warm to room temperature. The mixture was extracted with EtOAc $(3 \times 10 \mathrm{~mL})$, dried over $\mathrm{Na}_{2} \mathrm{SO}_{4}$, filtered and concentrated under reduced pressure. The mixture was purified by flash column chromatography to provide the title compound 7 as a colorless oil ( $32.5 \mathrm{mg}$, $57 \%$ yield, $\mathrm{dr}>20: 1,92 \%$ ee).

${ }^{1}$ H NMR (400 MHz, Chloroform- $d$ ) $\delta 7.32(\mathrm{~s}, 1 \mathrm{H}), 7.04(\mathrm{~s}, 1 \mathrm{H}), 6.93(\mathrm{dd}, J=8.2,1.9 \mathrm{~Hz}$, $1 \mathrm{H}), 6.70(\mathrm{dd}, J=8.1,1.3 \mathrm{~Hz}, 1 \mathrm{H}), 6.32-6.24(\mathrm{~m}, 1 \mathrm{H}), 6.05(\mathrm{~d}, J=3.1 \mathrm{~Hz}, 1 \mathrm{H}), 4.54(\mathrm{td}, J=$ 8.6, 1.4 Hz, 2H), 4.06 (td, $J=8.2,1.4 \mathrm{~Hz}, 1 \mathrm{H}), 3.97$ (ddtd, $J=9.1,7.1,5.7,1.4 \mathrm{~Hz}, 1 \mathrm{H}), 3.92$ - $3.85(\mathrm{~m}, 1 \mathrm{H}), 3.57-3.46(\mathrm{~m}, 1 \mathrm{H}), 3.18(\mathrm{t}, J=8.6 \mathrm{~Hz}, 2 \mathrm{H}), 2.75-2.66(\mathrm{~m}, 1 \mathrm{H}), 2.66-2.56$ (m, 1H), 2.37 (dddd, $J=12.1,7.4,5.8,1.4 \mathrm{~Hz}, 1 \mathrm{H}), 2.03-1.90(\mathrm{~m}, 1 \mathrm{H}), 1.86-1.69(\mathrm{~m}, 2 \mathrm{H}) .{ }^{13} \mathrm{C}$ NMR (101 MHz, $\left.\mathbf{C D C l}_{3}\right) \delta 158.3,155.9,141.5,134.0,127.9,127.1,125.0,110.2,109.1,104.7$, 79.4, 71.6, 71.3, 39.1, 38.0, 37.9, 32.1, 29.9 ppm; HRMS (ESI) calculated $[\mathrm{M}+\mathrm{H}]^{+}$for $\mathrm{C}_{18} \mathrm{H}_{21} \mathrm{O}_{3}{ }^{+}=285.1485$, found: $285.1488 .[\alpha]^{20}{ }_{\mathbf{D}}=-12.5\left(\mathrm{c}=0.20, \mathrm{CHCl}_{3}\right) . \mathbf{H P L C}$ analysis (ODH, $n$-Hexane: $i$-Propanol $=95: 5$ as eluent, $1 \mathrm{~mL} / \mathrm{min}, 220 \mathrm{~nm})$ indicated 92\% ee: $\mathrm{t}_{\mathrm{R}}($ minor $)=$ $7.1 \mathrm{~min}, \mathrm{t}_{\mathrm{R}}$ (major) $=7.7 \mathrm{~min}$.

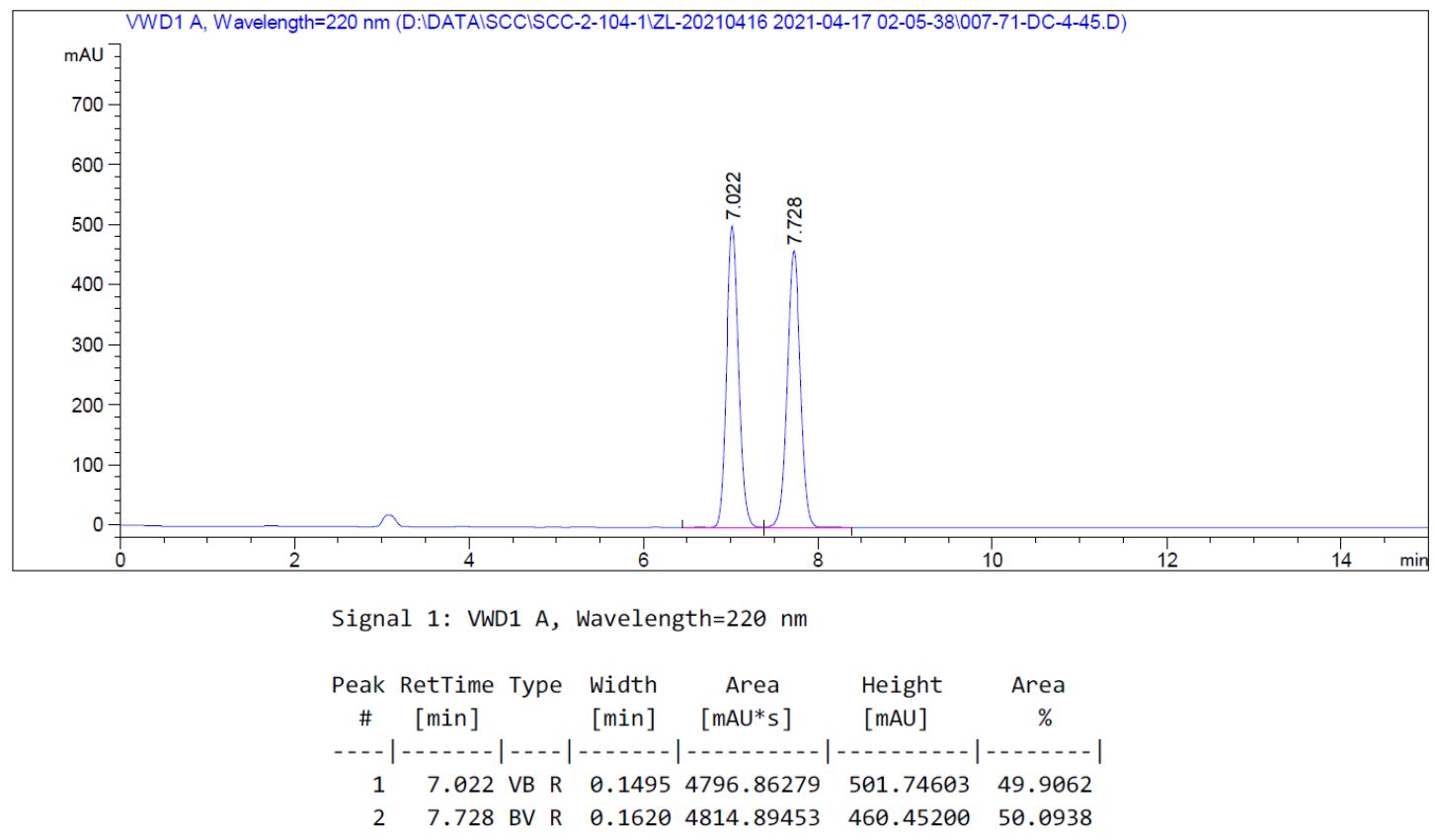




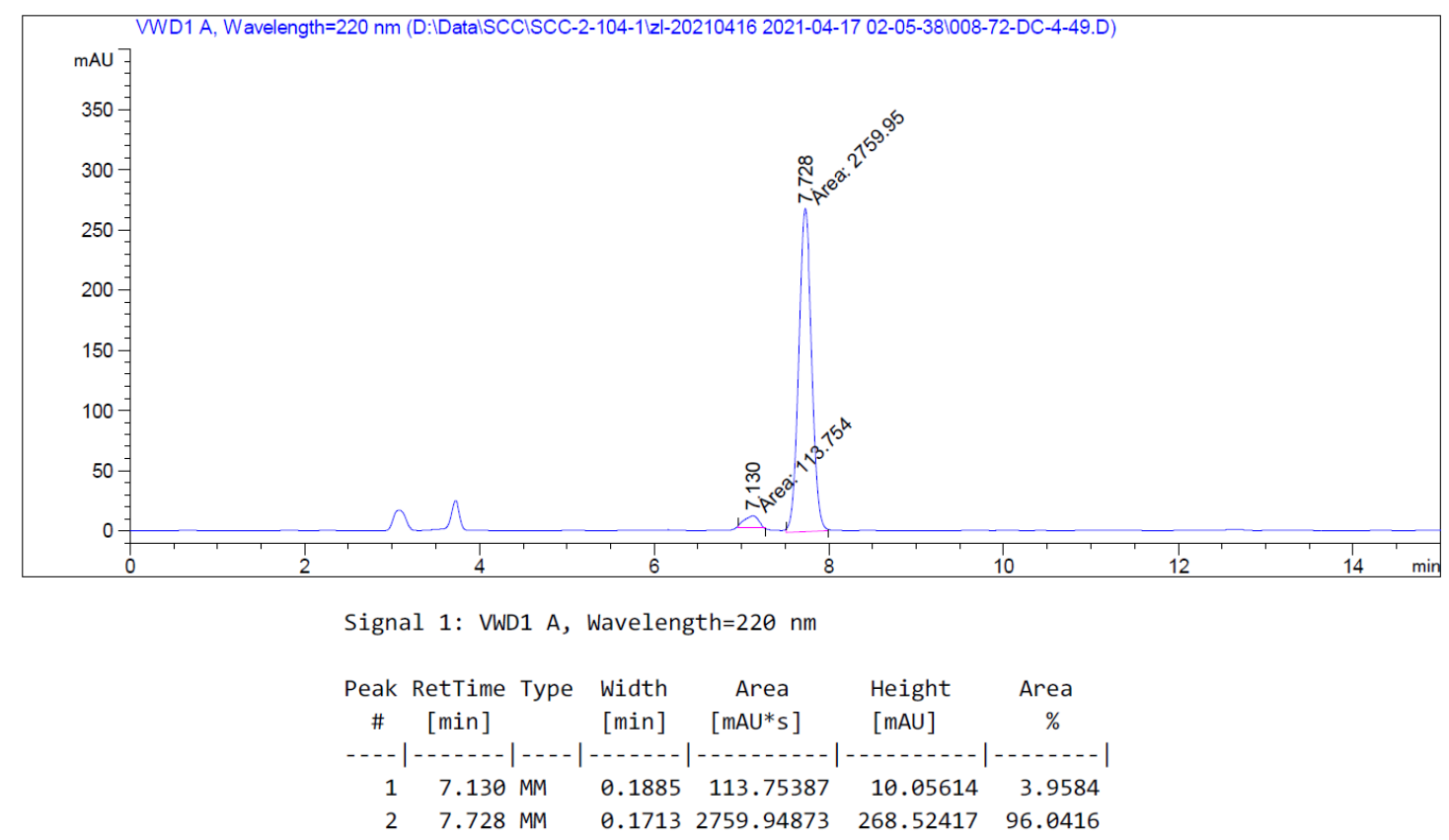

2-((3R,5R)-5-(2-(2,3-dihydrobenzofuran-5-yl)-ethyl)-tetrahydrofuran-3-yl)-1-methyl-1Hindole $^{6}$
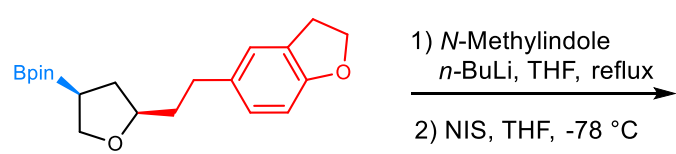

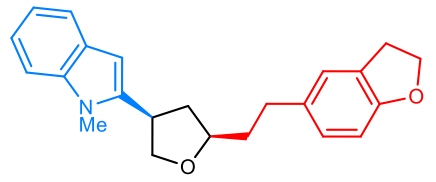

$$
8,65 \%
$$$$
\text { dr }>20: 1,95 \% \text { ee }
$$

To an oven-dried $10 \mathrm{~mL}$ reaction tube containing a stirring bar, a solution of $\mathrm{N}$ methylindole ( $52 \mu \mathrm{L}, 0.4 \mathrm{mmol}, 1.6$ equiv) in THF $(1.0 \mathrm{~mL})$ was treated $n$-BuLi $(0.12 \mathrm{~mL}, 0.3$ $\mathrm{mmol}, 2.5 \mathrm{M}$ in hexane) at room temprature. The mixture was heated at refluxed temperature for $5 \mathrm{~h}$. The reaction mixture was cooled to $-78{ }^{\circ} \mathrm{C}$ and a solution of compound $4 \mathbf{b a}$ (68.9 $\mathrm{mg}, 0.2 \mathrm{mmol}, 1.0$ equiv) in THF $(0.5 \mathrm{ml})$ was added dropwise. The resulting mixture was stirred at the same temperature for $2 \mathrm{~h}$. Then a solution of NIS (44.0 mg, $0.24 \mathrm{mmol}, 1.2$ equiv) in THF $(1.0 \mathrm{~mL})$ was added dropwise and stirred at $-78{ }^{\circ} \mathrm{C}$ for $1.5 \mathrm{~h}$. Saturated aqueous $\mathrm{Na}_{2} \mathrm{~S}_{2} \mathrm{O}_{3}$ solution $(2.0 \mathrm{~mL})$ was added and the reaction mixture was allowed to warm to room temperature. The mixture was extracted with EtOAc $(3 \times 10 \mathrm{~mL})$, dried over $\mathrm{Na}_{2} \mathrm{SO}_{4}$, filtered and concentrated under reduced pressure. The mixture was purified by flash column chromatography to provide the title compound 8 as a yellow oil (56.3mg, $65 \%$ yield, $\mathrm{dr}>20: 1$, $95 \%$ ee). 
${ }^{1}$ H NMR (400 MHz, Chloroform- $d$ ) $\delta 7.54(\mathrm{dt}, J=7.8,1.0 \mathrm{~Hz}, 1 \mathrm{H}), 7.27(\mathrm{dd}, J=8.2,1.0 \mathrm{~Hz}$, $1 \mathrm{H}), 7.17(\mathrm{ddd}, J=8.2,7.0,1.2 \mathrm{~Hz}, 1 \mathrm{H}), 7.12-7.01(\mathrm{~m}, 2 \mathrm{H}), 6.94(\mathrm{dd}, J=8.1,1.9 \mathrm{~Hz}, 1 \mathrm{H})$, $6.71(\mathrm{~d}, J=8.1 \mathrm{~Hz}, 1 \mathrm{H}), 6.34(\mathrm{t}, J=0.9 \mathrm{~Hz}, 1 \mathrm{H}), 4.54(\mathrm{t}, J=8.7 \mathrm{~Hz}, 2 \mathrm{H}), 4.23(\mathrm{t}, J=8.0 \mathrm{~Hz}$, 1H), 4.06 (ddt, $J=9.3,7.5,5.5 \mathrm{~Hz}, 1 \mathrm{H}), 3.91(\mathrm{t}, J=8.2 \mathrm{~Hz}, 1 \mathrm{H}), 3.69(\mathrm{~s}, 3 \mathrm{H}), 3.67-3.58(\mathrm{~m}$, 1H), $3.18(\mathrm{t}, J=8.7 \mathrm{~Hz}, 2 \mathrm{H}), 2.73(\mathrm{ddd}, J=13.8,9.7,5.7 \mathrm{~Hz}, 1 \mathrm{H}), 2.64(\mathrm{ddd}, J=13.8,9.4,6.6$ $\mathrm{Hz}, 1 \mathrm{H}), 2.49$ (ddd, $J=12.2,7.6,5.7 \mathrm{~Hz}, 1 \mathrm{H}), 1.99(\mathrm{dddd}, J=13.3,9.5,7.5,5.7 \mathrm{~Hz}, 1 \mathrm{H}), 1.89$ - $1.72(\mathrm{~m}, 2 \mathrm{H}) .{ }^{13} \mathbf{C}$ NMR (101 MHz, $\left.\mathbf{C D C l}_{3}\right) \delta$ 158.4, 141.4, 137.7, 133.9, 127.9, 127.7, 127.2, 125.0, 121.2, 120.2, 119.6, 109.1, 108.9, 97.6, 79.4, 72.2, 71.3, 39.0, 38.0, 37.7, 32.1, 29.9 ppm; HRMS (ESI) calculated $[\mathrm{M}+\mathrm{H}]^{+}$for $\mathrm{C}_{23} \mathrm{H}_{26} \mathrm{NO}_{2}{ }^{+}=348.1958$, found: $348.1958 .[\boldsymbol{\alpha}]^{\mathbf{2 0}}{ }_{\mathbf{D}}=-10.7$ $\left(\mathrm{c}=0.20, \mathrm{CHCl}_{3}\right)$. HPLC analysis (AD-H, $n$-Hexane: $i$-Propanol $=95: 5$ as eluent, $1 \mathrm{~mL} / \mathrm{min}$, $220 \mathrm{~nm}$ ) indicated $95 \%$ ee: $\mathrm{t}_{\mathrm{R}}$ (major) $=14.5 \mathrm{~min}, \mathrm{t}_{\mathrm{R}}($ minor $)=15.2 \mathrm{~min}$.
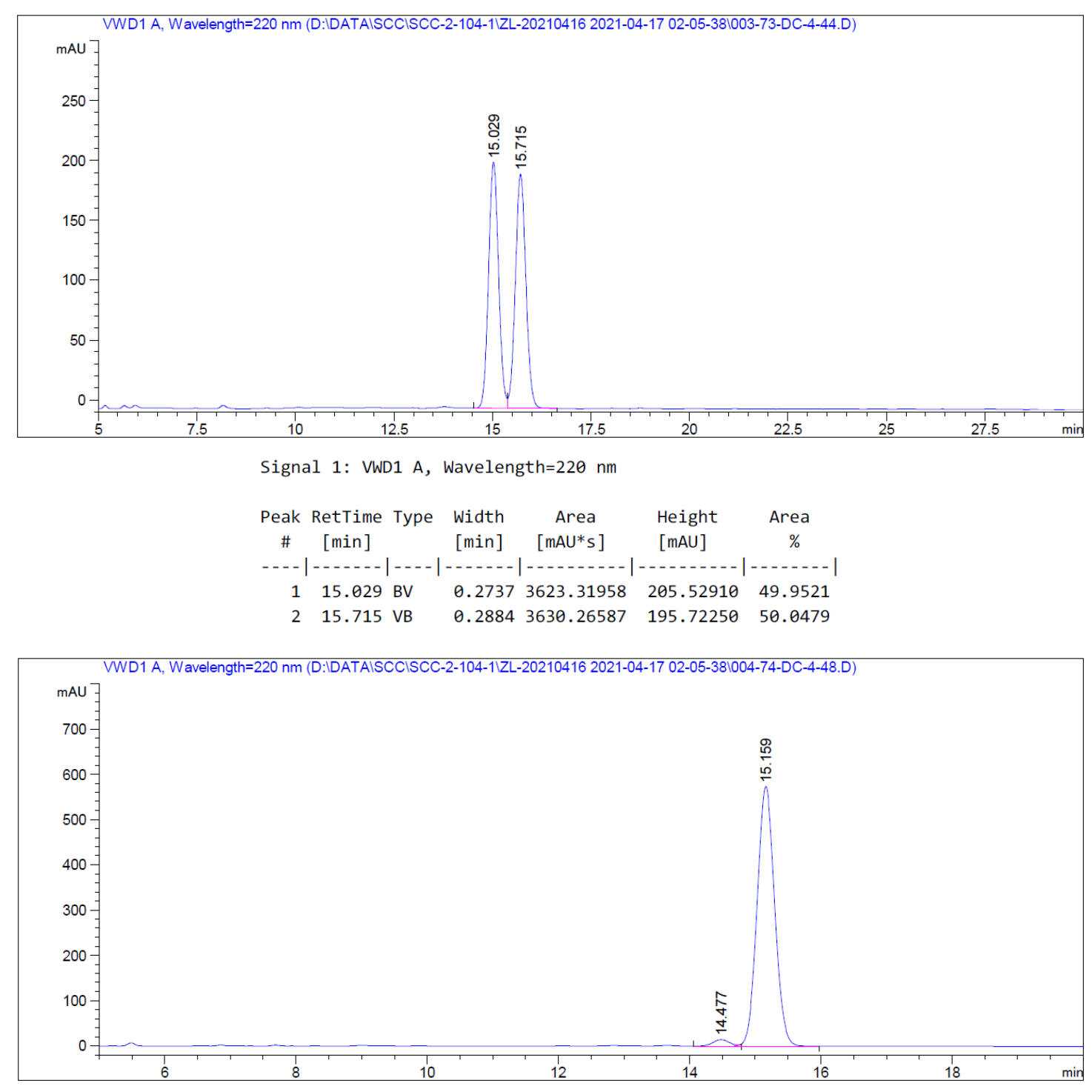


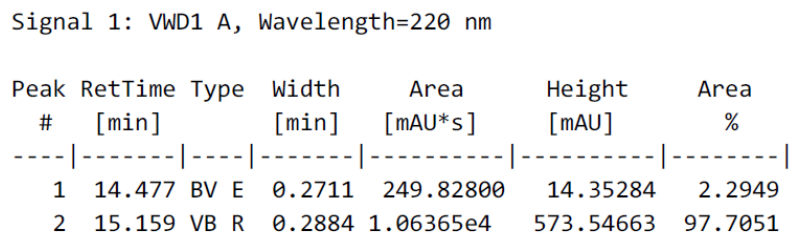

\section{Synthetic Applications}

cis-benzyl 2-(3,3-dimethylbutyl)-4-(hydroxymethyl)-piperidine-1-carboxylate ${ }^{7}$

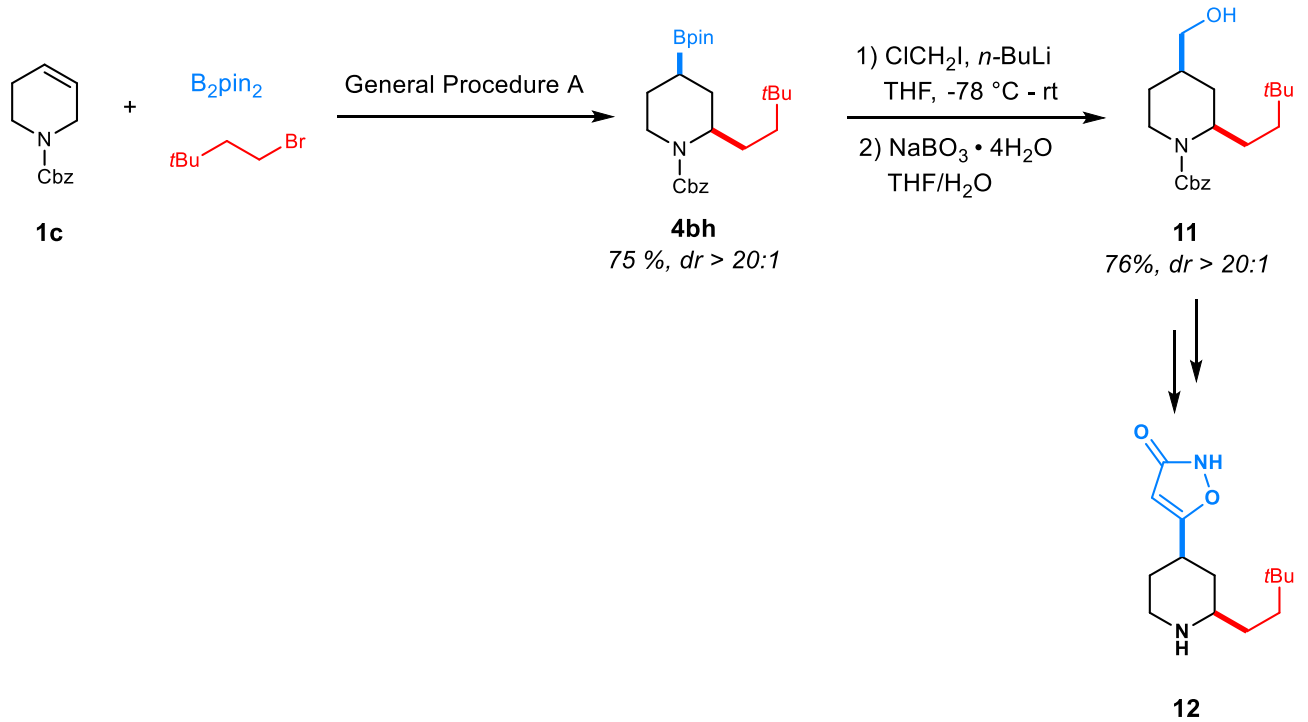

cis-benzyl 2-(3,3-dimethylbutyl)-4-(4,4,5,5-tetramethyl-1,3,2-dioxaborolan-2-yl)-piperidine1-carboxylate was synthesized according to general procedure A, obtained the pure compound 4bh as a colorless oil (129.1 mg, 75\% yield, dr > 20:1).

${ }^{1}$ H NMR (400 MHz, Chloroform-d) $\delta 7.36-7.32(\mathrm{~m}, 4 \mathrm{H}), 7.32-7.28(\mathrm{~m}, 1 \mathrm{H}), 5.17(\mathrm{~d}, J=$ $12.5 \mathrm{~Hz}, 1 \mathrm{H}), 5.08(\mathrm{~d}, J=12.4 \mathrm{~Hz}, 1 \mathrm{H}), 4.13-4.01(\mathrm{~m}, 1 \mathrm{H}), 3.96$ (ddd, $J=13.8,5.4,2.5 \mathrm{~Hz}$, 1H), $2.87(\mathrm{ddd}, J=13.7,12.1,3.9 \mathrm{~Hz}, 1 \mathrm{H}), 1.88(\mathrm{dt}, J=13.6,6.1 \mathrm{~Hz}, 1 \mathrm{H}), 1.76-1.69(\mathrm{~m}, 1 \mathrm{H})$, $1.67-1.64(\mathrm{~m}, 2 \mathrm{H}), 1.60-1.56(\mathrm{~m}, 1 \mathrm{H}), 1.36-1.28(\mathrm{~m}, 1 \mathrm{H}), 1.25(\mathrm{~d}, J=1.6 \mathrm{~Hz}, 12 \mathrm{H}), 1.22$ $1.16(\mathrm{~m}, 2 \mathrm{H}), 1.11-1.07(\mathrm{~m}, 1 \mathrm{H}), 0.83(\mathrm{~s}, 9 \mathrm{H}) \mathrm{ppm} ;{ }^{13} \mathbf{C} \mathbf{N M R}\left(\mathbf{1 0 1} \mathbf{M H z}, \mathbf{C D C l}_{3}\right) \delta$ 156.0, $137.4,128.5,127.89,127.87,83.4,66.9,53.2,40.8,38.1,30.2,29.8,29.5,27.3,25.8,25.1$, 25.0 ppm; ${ }^{11}$ B NMR (128 MHz, Chloroform-d) $\delta 33.91$ ppm; HRMS (ESI) calculated [M+H] ${ }^{+}$ for $\mathrm{C}_{25} \mathrm{H}_{41} \mathrm{BNO}_{4}{ }^{+}=430.3123$, found: 430.3116 . 
To an oven-dried round bottom flask containing a stirring bar was added a solution of $\mathbf{4 b h}$ ( 45.0 $\mathrm{mg}, 0.1 \mathrm{mmol}, 1.0$ equiv) and iodochloromethane (15.2 $\mu \mathrm{L}, 0.2 \mathrm{mmol}, 2.0$ equiv.) in THF (1.0 $\mathrm{mL}$ ) under $\mathrm{N}_{2}$ atmosphere. The reaction mixture was cooled to $-78{ }^{\circ} \mathrm{C} . n$-BuLi $(0.08 \mathrm{~mL}, 0.2$ mmol, 2.0 equiv, 2.5 $\mathrm{M}$ in hexane) was slowly added and the solution was stirred for $10 \mathrm{~min}$ at the same temperature. The reaction mixture was warmed to room temperature and additionally stirred for $20 \mathrm{~h}$. Then the reaction was quenched with saturated aqueous solution of $\mathrm{NaCl}(5$ $\mathrm{mL})$ and extracted with EtOAc $(3 \times 5 \mathrm{~mL})$, dried over $\mathrm{Na}_{2} \mathrm{SO}_{4}$, concentrated under reduced pressure. Then the crude material and $\mathrm{NaBO}_{3} \cdot 4 \mathrm{H}_{2} \mathrm{O}(63.4 \mathrm{mg}, 0.4 \mathrm{mmol}, 4.0$ equiv) were dissolved in $\mathrm{THF} / \mathrm{H}_{2} \mathrm{O}(1 \mathrm{~mL}, 1: 1)$. After stirred at ambient temperature $16 \mathrm{~h}$, the reaction was quenched by saturated aqueous solution of $\mathrm{NaCl}(5 \mathrm{~mL})$. The mixture was extracted with EtOAc, dried over $\mathrm{Na}_{2} \mathrm{SO}_{4}$, concentrated under reduced pressure. The mixture was purified by flash column chromatography to provide the title compound $\mathbf{1 1}$ as a colorless oil $(25.2 \mathrm{mg}, 76 \%$ yield, $\mathrm{dr}>20: 1)$.

${ }^{1}$ H NMR (400 MHz, Chloroform-d) $\delta 7.37$ - 7.27 (m, 5H), 5.18 (d, J=12.4 Hz, 1H), 5.08 (d, $J=12.4 \mathrm{~Hz}, 1 \mathrm{H}), 3.88(\mathrm{ddt}, J=13.4,7.9,2.7 \mathrm{~Hz}, 2 \mathrm{H}), 3.61(\mathrm{dd}, J=10.5,6.2 \mathrm{~Hz}, 1 \mathrm{H}), 3.49$ $(\mathrm{dd}, J=10.5,6.6 \mathrm{~Hz}, 1 \mathrm{H}), 3.04(\mathrm{ddd}, J=14.0,10.6,6.0 \mathrm{~Hz}, 1 \mathrm{H}), 1.96-1.83(\mathrm{~m}, 2 \mathrm{H}), 1.81$ $1.70(\mathrm{~m}, 1 \mathrm{H}), 1.63-1.57(\mathrm{~m}, 1 \mathrm{H}), 1.47(\mathrm{~s}, 1 \mathrm{H}), 1.44-1.35(\mathrm{~m}, 1 \mathrm{H}), 1.29$ (dddd, $J=12.9,8.3$, 5.9, $2.6 \mathrm{~Hz}, 1 \mathrm{H}), 1.23$ - $1.12(\mathrm{~m}, 3 \mathrm{H}), 0.84(\mathrm{~s}, 9 \mathrm{H}) .{ }^{13} \mathbf{C}$ NMR (101 MHz, $\left.\mathbf{C D C l}_{\mathbf{3}}\right) \delta$ 156.1, 137.2, 128.6, 128.0, 127.9, 67.0, 66.9, 54.0, 40.1 , 37.4, 34.3, 30.3, 30.2, 29.8, 29.5, 25.7 ppm; HRMS (ESI) calculated $[\mathrm{M}+\mathrm{H}]^{+}$for $\mathrm{C}_{20} \mathrm{H}_{32} \mathrm{NO}_{3}{ }^{+}=334.2377$, found: 334.2380 .

cis-ethyl 4-(6-(3,4-dimethoxyphenyl)-4-hydroxypiperidin-2-yl)-butanoate ${ }^{8}$ 


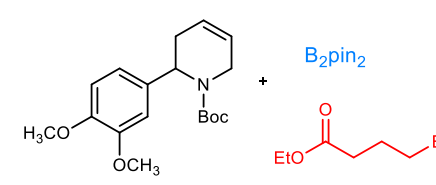

$1 d$

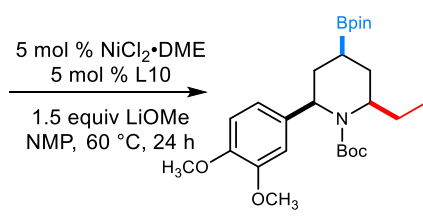

46. 4 bi

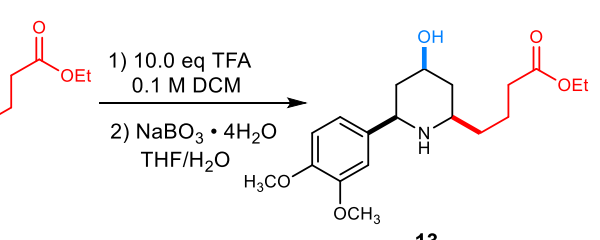

$70 \%, d r>20: 1$

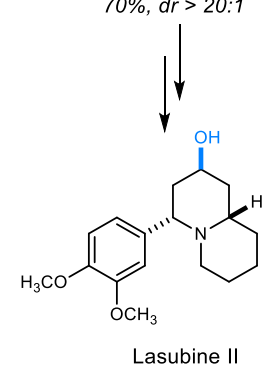

cis-tert-butyl 2-(3,4-dimethoxyphenyl)-6-(4-ethoxy-4-oxobutyl)-4-(4,4,5,5-tetramethyl-1,3,2dioxaborolan-2-yl)-piperidine-1-carboxylate was synthesized according to general procedure A $\left(60{ }^{\circ} \mathrm{C}, 24 \mathrm{~h}\right)$, obtained the pure compound $4 \mathbf{b i}$ as a colorless oil $(143.2 \mathrm{mg}, 64 \%$ yield, $\mathrm{dr}>$ 20:1).

${ }^{1}$ H NMR (400 MHz, Chloroform-d) $\delta 6.83-6.72(\mathrm{~m}, 3 \mathrm{H}), 5.07$ (s, 1H), $4.12(\mathrm{q}, J=7.1 \mathrm{~Hz}$, 2H), $3.91-3.87(\mathrm{~m}, 1 \mathrm{H}), 3.86(\mathrm{~s}, 3 \mathrm{H}), 3.85(\mathrm{~s}, 3 \mathrm{H}), 2.36-2.28(\mathrm{~m}, 2 \mathrm{H}), 2.19$ - $2.07(\mathrm{~m}, 2 \mathrm{H})$, $1.90(\mathrm{ddd}, J=14.6,9.9,4.9 \mathrm{~Hz}, 1 \mathrm{H}), 1.79-1.62(\mathrm{~m}, 4 \mathrm{H}), 1.58-1.46(\mathrm{~m}, 1 \mathrm{H}), 1.36(\mathrm{~s}, 9 \mathrm{H})$, $1.27-1.21(\mathrm{~m}, 15 \mathrm{H}), 1.16-1.06(\mathrm{~m}, 1 \mathrm{H}) .{ }^{13} \mathbf{C}$ NMR (101 MHz, $\left.\mathbf{C D C l}_{3}\right) \delta 173.7,155.8,148.8$, $147.4,117.9,110.9,109.4,83.4,79.4,60.3,56.0,55.89,54.88,52.0,36.0,34.5,28.5,25.3$, 24.9, 24.7, 22.6, 14.3 ppm; ${ }^{11}$ B NMR (128 MHz, Chloroform-d) $\delta 34.97$ ppm; HRMS (ESI) calculated $[\mathrm{M}+\mathrm{H}]^{+}$for $\mathrm{C}_{30} \mathrm{H}_{49} \mathrm{BNO}_{8}{ }^{+}=562.3546$, found: 562.3539 .

To an oven-dried $10 \mathrm{~mL}$ reaction tube containing a stirring bar, the product $\mathbf{4 b i}(56.1 \mathrm{mg}, 0.1$ mmol, 1.0 equiv) was dissolved in $\mathrm{CH}_{2} \mathrm{Cl}_{2}(1 \mathrm{~mL})$. Then trifluoroacetic acid (76 $\mu \mathrm{L}, 1.0 \mathrm{mmol}$, 10.0 equiv) was added dropwise at $0{ }^{\circ} \mathrm{C}$ and the mixture was stirred at the same temperature for $15 \mathrm{~min}$. The solution was allowed to warm up to room temperature and stirred. After $1 \mathrm{~h}$, the mixture was concentrated under reduced pressure. The crude material and $\mathrm{NaBO}_{3} \cdot 4 \mathrm{H}_{2} \mathrm{O}(63.4$ $\mathrm{mg}, 0.4 \mathrm{mmol}, 4.0$ equiv) were dissolved in $\mathrm{THF} / \mathrm{H}_{2} \mathrm{O}(1 \mathrm{~mL}, 1: 1)$. After stirred at ambient temperature $16 \mathrm{~h}$, the reaction was quenched by saturated aqueous solution of $\mathrm{NaCl}(5 \mathrm{~mL})$. The mixture was extracted with EtOAc, dried over $\mathrm{Na}_{2} \mathrm{SO}_{4}$, concentrated under reduced pressure. The mixture was purified by flash column chromatography to provide the title compound $\mathbf{1 3}$ as a colorless oil (24.5 mg, 70\% yield, $\mathrm{dr}>20: 1)$. 
${ }^{1}$ H NMR (400 MHz, Chloroform- $d$ ) $\delta 6.94(\mathrm{~d}, J=2.0 \mathrm{~Hz}, 1 \mathrm{H}), 6.89(\mathrm{dd}, J=8.1,2.0 \mathrm{~Hz}, 1 \mathrm{H})$, $6.82(\mathrm{~d}, J=8.2 \mathrm{~Hz}, 1 \mathrm{H}), 4.12(\mathrm{q}, J=7.1 \mathrm{~Hz}, 2 \mathrm{H}), 3.89(\mathrm{~s}, 3 \mathrm{H}), 3.87(\mathrm{~s}, 3 \mathrm{H}), 3.83-3.73(\mathrm{~m}$, 1H), $3.61(\mathrm{dd}, J=11.5,2.4 \mathrm{~Hz}, 1 \mathrm{H}), 2.72(\mathrm{dtd}, J=11.1,6.3,2.3 \mathrm{~Hz}, 1 \mathrm{H}), 2.32(\mathrm{t}, J=7.4 \mathrm{~Hz}$, 2H), $2.13-2.00(\mathrm{~m}, 2 \mathrm{H}), 1.73-1.64(\mathrm{~m}, 2 \mathrm{H}), 1.54-1.43(\mathrm{~m}, 3 \mathrm{H}), 1.25(\mathrm{t}, J=7.2 \mathrm{~Hz}, 3 \mathrm{H}), 1.17$ $(\mathrm{q}, J=11.3 \mathrm{~Hz}, 1 \mathrm{H}){ }^{13} \mathbf{C} \mathbf{N M R}\left(\mathbf{1 0 1} \mathbf{M H z}, \mathbf{C D C l}_{3}\right) \delta 173.7,149.1,148.4,136.7,118.9,111.2$, 110.2, 69.7, 60.5, 59.6, 56.1, 56.0, 55.1, 43.8, 41.4, 36.2, 34.4, 21.5, 14.4 ppm; HRMS (ESI) calculated $[\mathrm{M}+\mathrm{H}]^{+}$for $\mathrm{C}_{19} \mathrm{H}_{30} \mathrm{NO}_{5}{ }^{+}=352.2119$, found: 352.2113 .

\section{Mechanism Studies}

\subsection{Radical Clock Experiment}

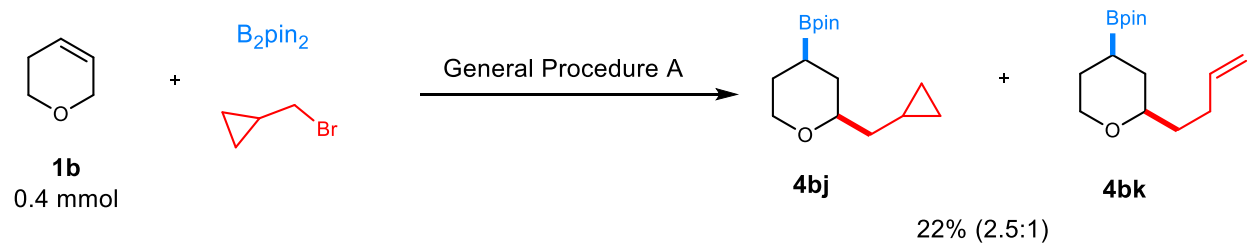

Following the General Procedure A, the crude product was purified by flash column chromatography on silica gel to afford the mixture of $\mathbf{4 b j}$ and $\mathbf{4 b k}$ as a colorless oil (23.4 $\mathrm{mg}$, $22 \%)$.

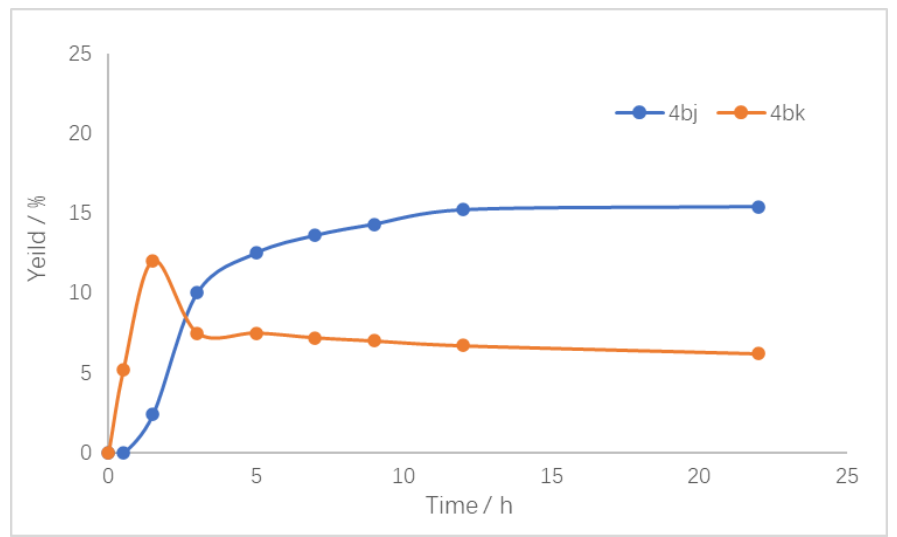

Fig S2. Time Course of the Reaction 


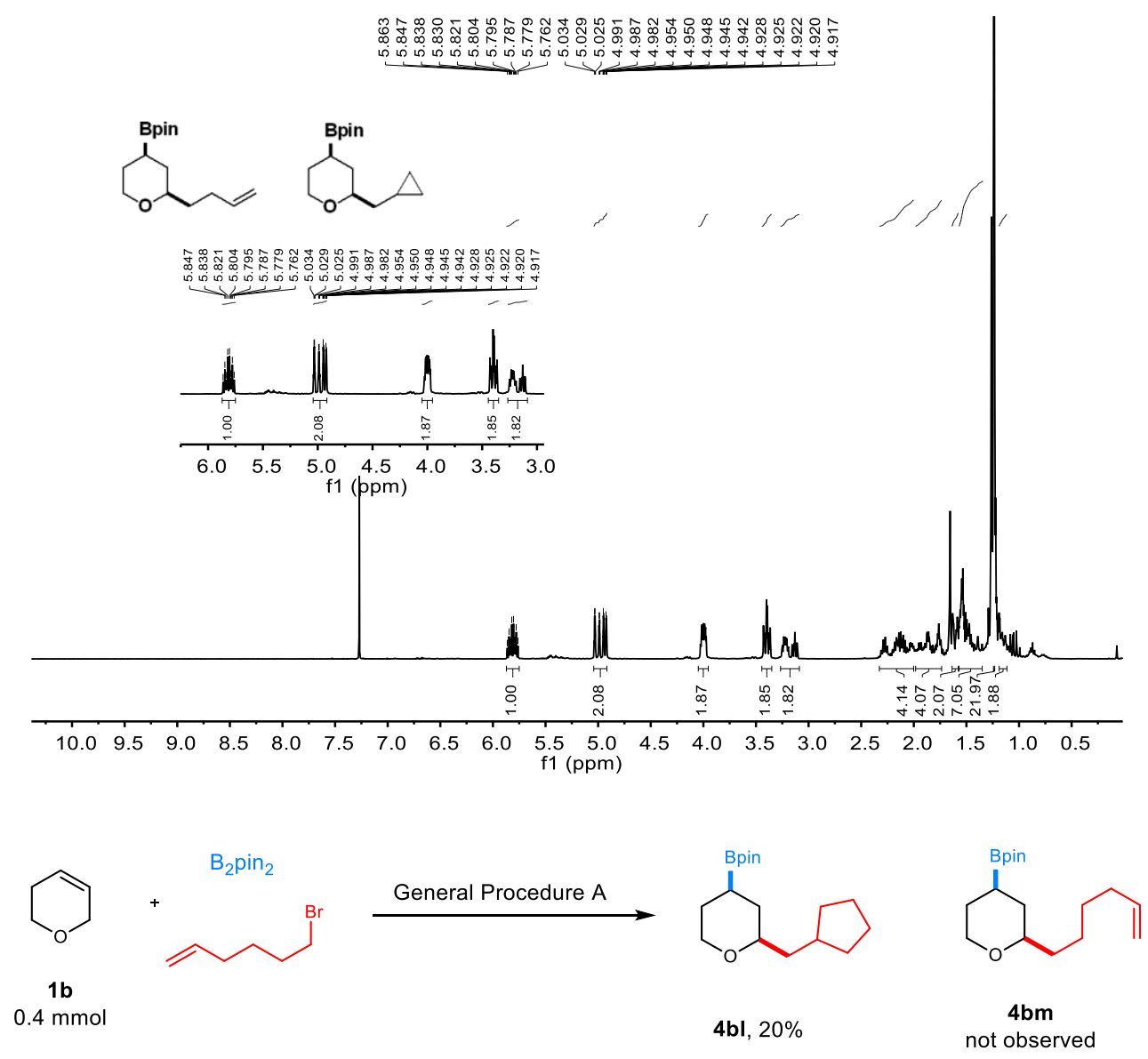

Following the General Procedure A, the crude product was purified by flash column chromatography on silica gel to afford $\mathbf{4 b l}$ as a colorless oil $(23.8 \mathrm{mg}, 20 \%$, dr $>20: 1)$.

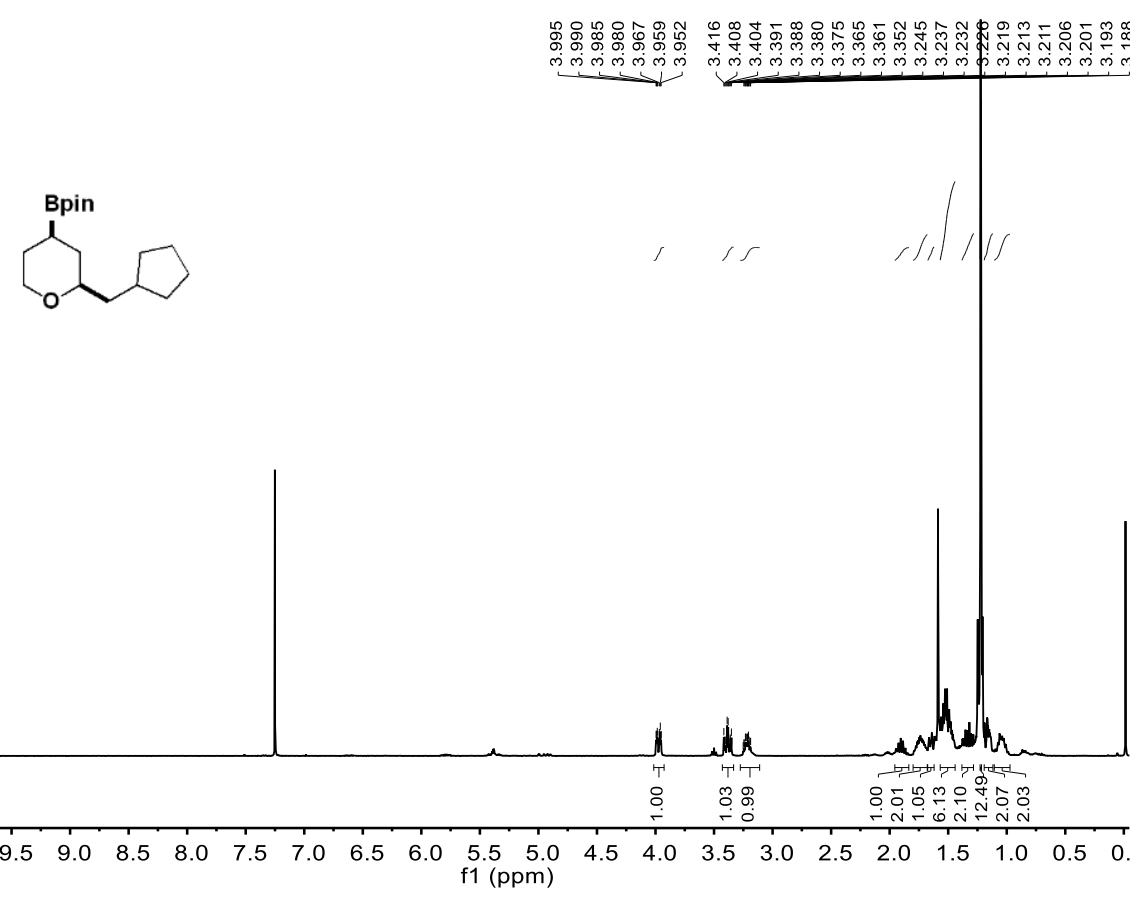




\subsection{Reaction with Bromobenzene}

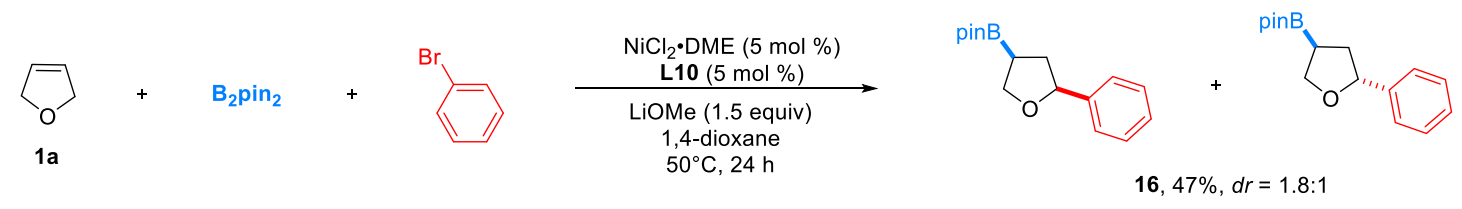

Under $\mathrm{N}_{2}$ atmosphere, an oven-dried $10 \mathrm{~mL}$ reaction tube which equipped with a magnetic stir bar and sealed with a rubber stopper sequentially was added $\mathrm{NiCl}_{2} \cdot \mathrm{DME}(4.4 \mathrm{mg}, 0.02 \mathrm{mmol}$, $5 \mathrm{~mol} \%$ ), L10 (6.0 mg, $0.02 \mathrm{mmol}, 5 \mathrm{~mol} \%$ ), LiOMe (22.8 mg, $0.6 \mathrm{mmol}, 1.5$ equiv) and bis(pinacolato)diboron (152.4 mg, $0.6 \mathrm{mmol}, 1.5$ equiv). Then anhydrous 1,4-dioxane ( $1 \mathrm{~mL})$, alkenes ( $0.4 \mathrm{mmol}, 1.0$ equiv), bromobenzene ( $0.6 \mathrm{mmol}, 1.5$ equiv), anhydrous 1,4 -dioxane (1 $\mathrm{mL}$ ) were added and the mixture was stirred. After $24 \mathrm{~h}$ of stirring at $50{ }^{\circ} \mathrm{C}$, the resulting mixture was quenched with water $(3 \mathrm{~mL})$ and further diluted with ethyl acetate $(3 \mathrm{~mL})$. Then the mixture was extracted with ethyl acetate and the combined organic layers were dried over anhydrous $\mathrm{Na}_{2} \mathrm{SO}_{4}$, filtered, and concentrated under vacuum. The crude material was separated on a silica gel column affording the desired product as a colorless oil $(51.0 \mathrm{mg}, 47 \%, \mathrm{dr}=1.8: 1)$.

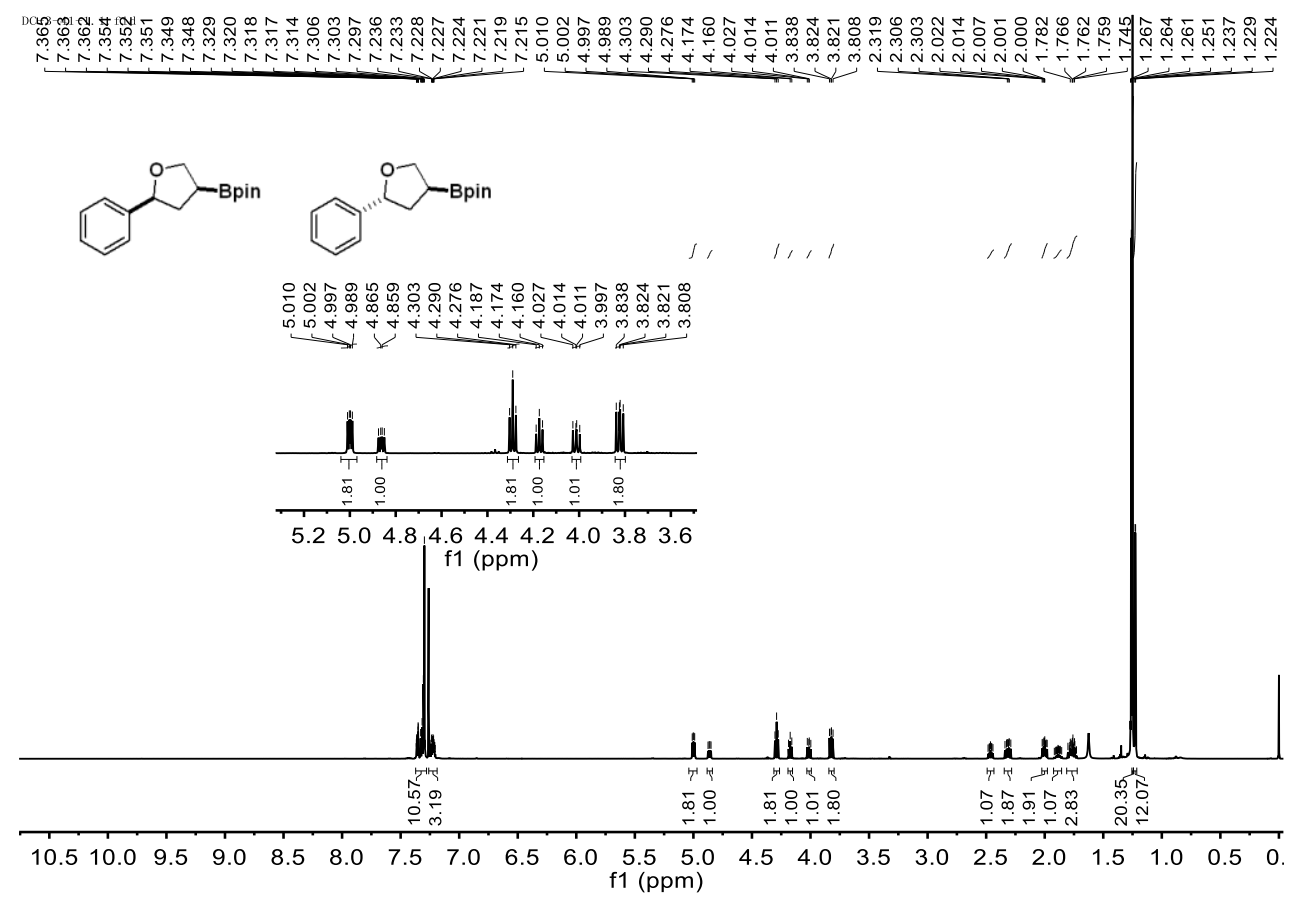




\section{X-ray Structures and Data}

Crystals suitable for X-ray single-crystal diffraction analysis were obtained from $\mathrm{Hexane} / \mathrm{EtO}_{2}$ using slow evaporation under air at room temperature.

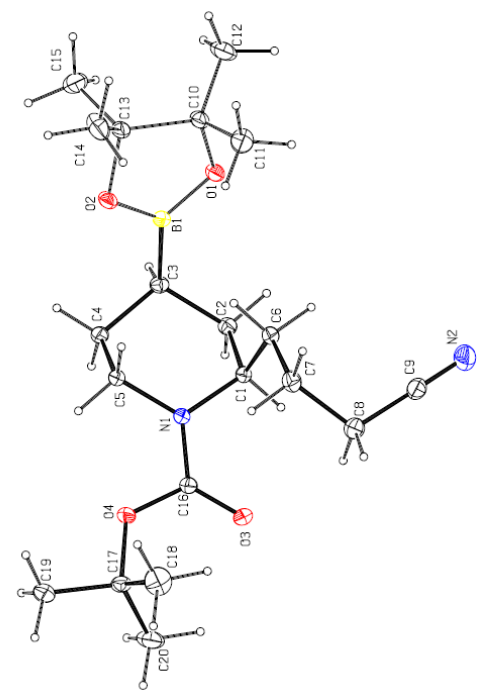

CCDC: 2102385

Table 1. Crystal data and structure refinement for Tab.

Identification code

Empirical formula

Formula weight

Temperature

Wavelength

Crystal system

Space group

Unit cell dimensions

Volume

Z

Density (calculated)

Absorption coefficient

$\mathrm{F}(000)$

Crystal size

Theta range for data collection

Index ranges

Reflections collected

Independent reflections

$$
\begin{aligned}
& \text { 210318b_0m_pl } \\
& \text { C20 H35 B N2 O4 }
\end{aligned}
$$

378.31

100(1) K

$1.34139 \AA$

Triclinic

P-1

$\mathrm{a}=6.5174(4) \AA$

$\alpha=65.948(2)^{\circ}$.

$\mathrm{b}=12.8864(9) \AA$

$\beta=77.709(2)^{\circ}$.

$\mathrm{c}=14.9099(10) \AA$

$\gamma=78.441(2)^{\circ}$.

$1108.19(13) \AA^{3}$

2

$1.134 \mathrm{Mg} / \mathrm{m}^{3}$

$0.394 \mathrm{~mm}^{-1}$

412

$0.12 \times 0.1 \times 0.1 \mathrm{~mm}^{3}$

2.854 to $55.000^{\circ}$.

$-7<=\mathrm{h}<=7,-15<=\mathrm{k}<=15,-18<=1<=18$

38051

$4159[\mathrm{R}($ int $)=0.0469]$ 
Completeness to theta $=53.594^{\circ}$

Absorption correction

Max. and min. transmission

Refinement method

Data / restraints / parameters

Goodness-of-fit on $\mathrm{F}^{2}$

Final $R$ indices [I>2sigma(I)]

$\mathrm{R}$ indices (all data)

Extinction coefficient

Largest diff. peak and hole
$98.8 \%$

Semi-empirical from equivalents

1.0000 and 0.8942

Full-matrix least-squares on $\mathrm{F}^{2}$

4159 / 29 / 281

1.106

$\mathrm{R} 1=0.0716, \mathrm{wR} 2=0.2242$

$\mathrm{R} 1=0.0782, \mathrm{wR} 2=0.2293$

$0.033(5)$

0.408 and -0.241 e. $\AA^{-3}$

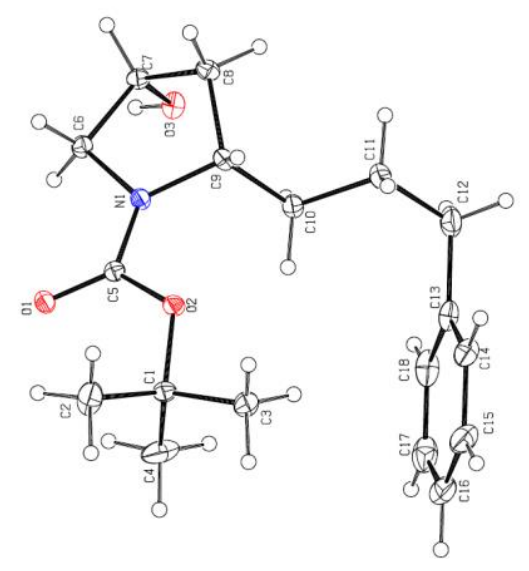

CCDC: 2102398

Table 1. Crystal data and structure refinement for 210318C_0M.

Identification code

Empirical formula

Formula weight

Temperature

Wavelength

Crystal system

Space group

Unit cell dimensions

Volume

$\mathrm{Z}$

Density (calculated)

Absorption coefficient

$\mathrm{F}(000)$

Crystal size 210318c_0m

C18 H27 N O3

305.40

$100.0 \mathrm{~K}$

$1.34138 \AA$

Orthorhombic

$\mathrm{P} 212121$

$$
\begin{array}{ll}
\mathrm{a}=6.0094(3) \AA & \alpha=90^{\circ} . \\
\mathrm{b}=9.1334(5) \AA & \beta=90^{\circ} . \\
\mathrm{c}=31.7659(16) \AA & \gamma=90^{\circ} .
\end{array}
$$$$
\text { 1743.51(16) } \AA^{3}
$$

4

$$
1.163 \mathrm{Mg} / \mathrm{m}^{3}
$$

$0.398 \mathrm{~mm}^{-1}$

664

$0.2 \times 0.2 \times 0.1 \mathrm{~mm}^{3}$ 
Theta range for data collection

Index ranges

Reflections collected

Independent reflections

Completeness to theta $=53.594^{\circ}$

Absorption correction

Max. and min. transmission

Refinement method

Data / restraints / parameters

Goodness-of-fit on $\mathrm{F}^{2}$

Final R indices [I $>2 \operatorname{sigma}(\mathrm{I})]$

$\mathrm{R}$ indices (all data)

Absolute structure parameter

Extinction coefficient

Largest diff. peak and hole
4.382 to $58.609^{\circ}$.

$-7<=\mathrm{h}<=7,-11<=\mathrm{k}<=11,-40<=\mathrm{l}<=40$

19280

$3710[\mathrm{R}(\mathrm{int})=0.0316]$

$97.8 \%$

Semi-empirical from equivalents

0.7515 and 0.6861

Full-matrix least-squares on $\mathrm{F}^{2}$

$3710 / 0 / 203$

1.045

$\mathrm{R} 1=0.0279, \mathrm{wR} 2=0.0740$

$\mathrm{R} 1=0.0280, \mathrm{wR} 2=0.0741$

$0.29(4)$

$\mathrm{n} / \mathrm{a}$

0.193 and -0.124 e. $\AA^{-3}$

CCDC: 2102400

Table 1. Crystal data and structure refinement for table.

Identification code

Empirical formula

Formula weight

Temperature

Wavelength

Crystal system

Space group

Unit cell dimensions

Volume

Z

Density (calculated) 210322b_0ma_pl

C13 H18 O3

222.27

$100.15 \mathrm{~K}$

$1.34138 \AA$

Orthorhombic

$\mathrm{P} 2{ }_{12} 2_{1}$

$\mathrm{a}=6.4943(4) \AA$

$\alpha=90^{\circ}$.

$\mathrm{b}=6.5895(5) \AA$

$\beta=90^{\circ}$.

$\mathrm{c}=27.0102(18) \AA$

$\gamma=90^{\circ}$.
1155.88(14) $\AA^{3}$

4

$1.277 \mathrm{Mg} / \mathrm{m}^{3}$ 
Absorption coefficient

$\mathrm{F}(000)$

Crystal size

Theta range for data collection

Index ranges

Reflections collected

Independent reflections

Completeness to theta $=53.594^{\circ}$

Absorption correction

Max. and min. transmission

Refinement method

Data / restraints / parameters

Goodness-of-fit on $\mathrm{F}^{2}$

Final $\mathrm{R}$ indices [I $>2 \operatorname{sigma}(\mathrm{I})]$

$\mathrm{R}$ indices (all data)

Absolute structure parameter

Extinction coefficient

Largest diff. peak and hole
$0.461 \mathrm{~mm}^{-1}$

480

$0.1 \times 0.1 \times 0.08 \mathrm{~mm}^{3}$

5.700 to $61.801^{\circ}$.

$-8<=\mathrm{h}<=7,-8<=\mathrm{k}<=8,-35<=1<=35$

10093

$2322[\mathrm{R}(\mathrm{int})=0.0308]$

$83.9 \%$

Semi-empirical from equivalents

1.0000 and 0.8812

Full-matrix least-squares on $\mathrm{F}^{2}$

$2322 / 0 / 147$

1.109

$\mathrm{R} 1=0.0344, \mathrm{wR} 2=0.0929$

$\mathrm{R} 1=0.0346, \mathrm{wR} 2=0.0930$

$0.1(2)$

$\mathrm{n} / \mathrm{a}$

0.195 and -0.151 e. $\AA^{-3}$ 


\section{NMR Spectra}
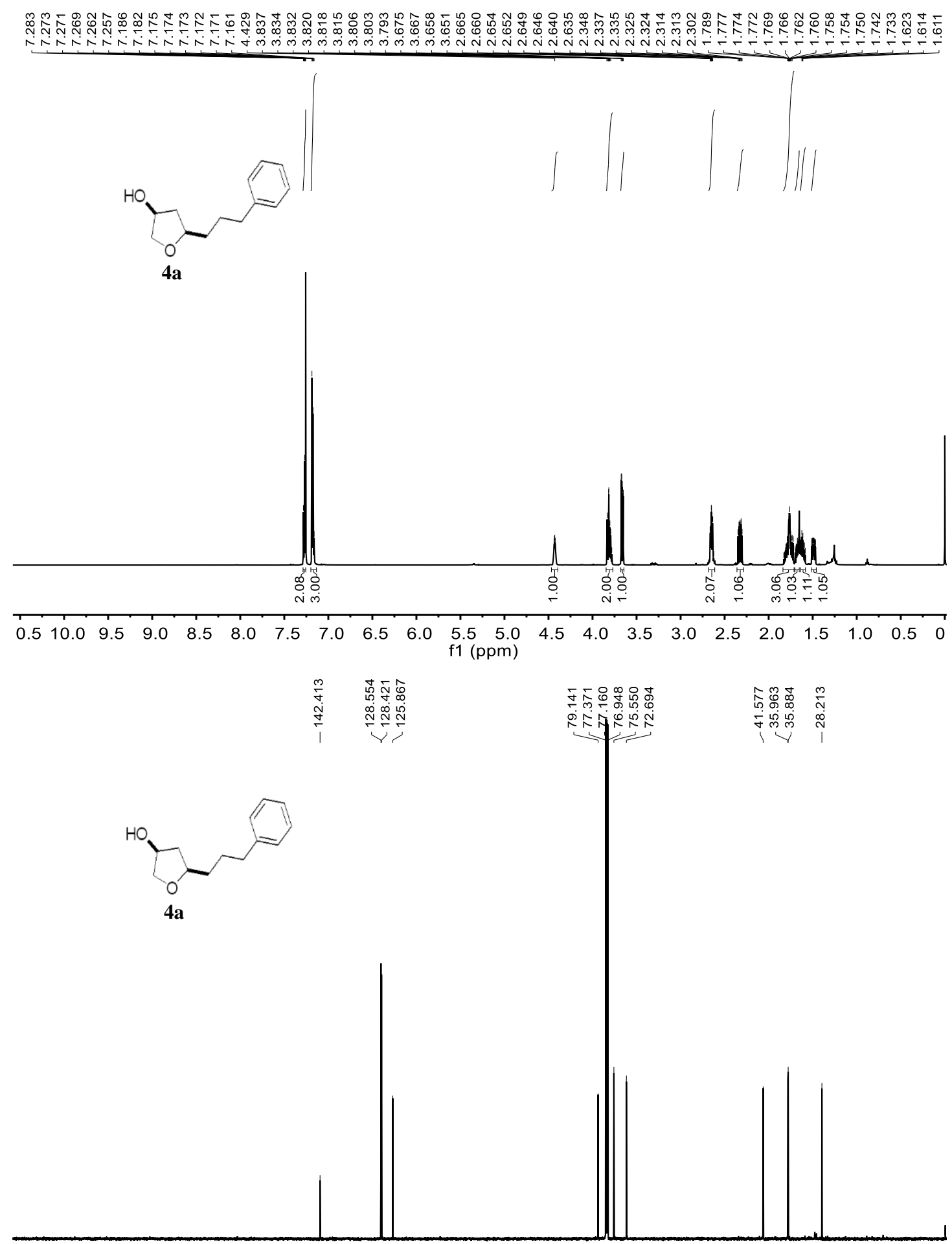

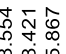

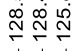

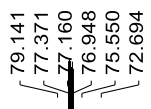

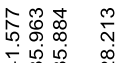

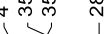

$\begin{array}{lllllllllllllllllllll}210 & 200 & 190 & 180 & 170 & 160 & 150 & 140 & 130 & 120 & 110 & 100 & 90 & 80 & 70 & 60 & 50 & 40 & 30 & 20 & 10\end{array}$ f1 (ppm) 
<smiles>CCOC(=O)CCCC1CC(Cc2ccccc2)CO1</smiles>
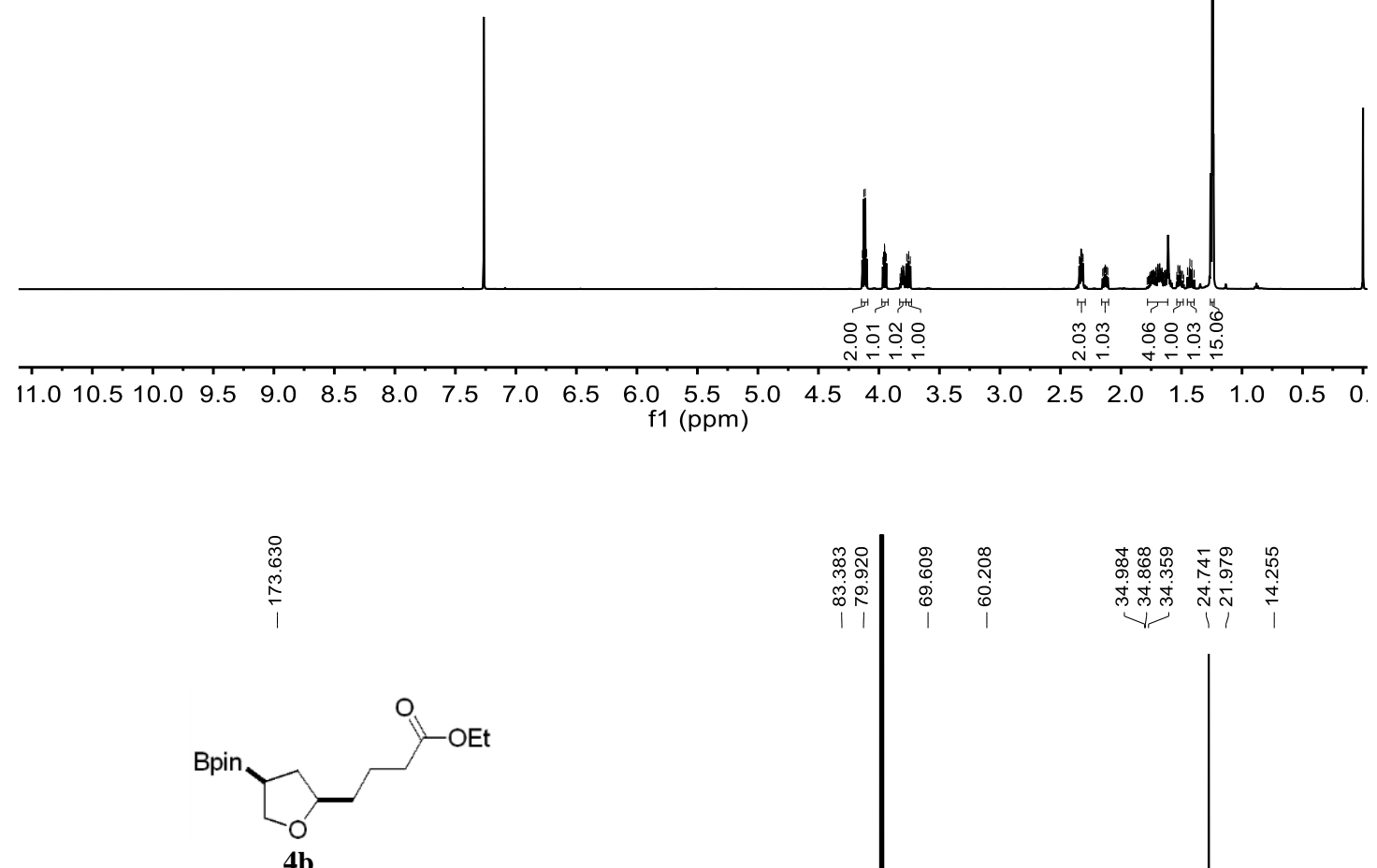

4b

(1)

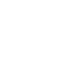




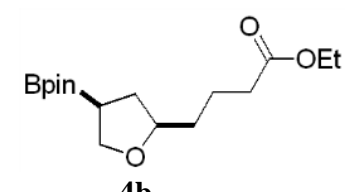

$4 b$

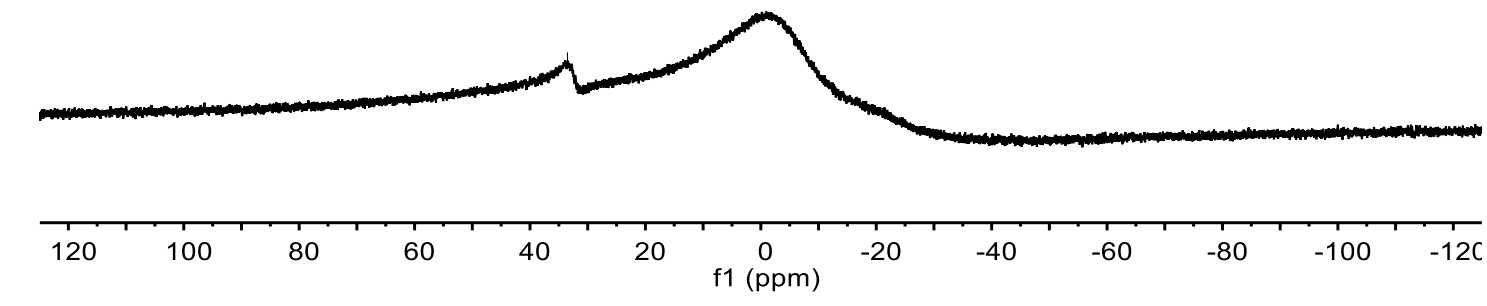

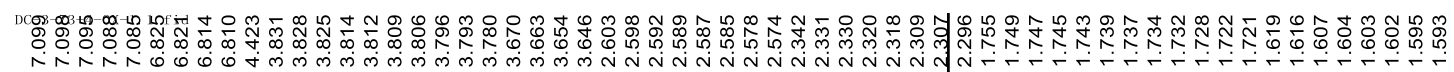

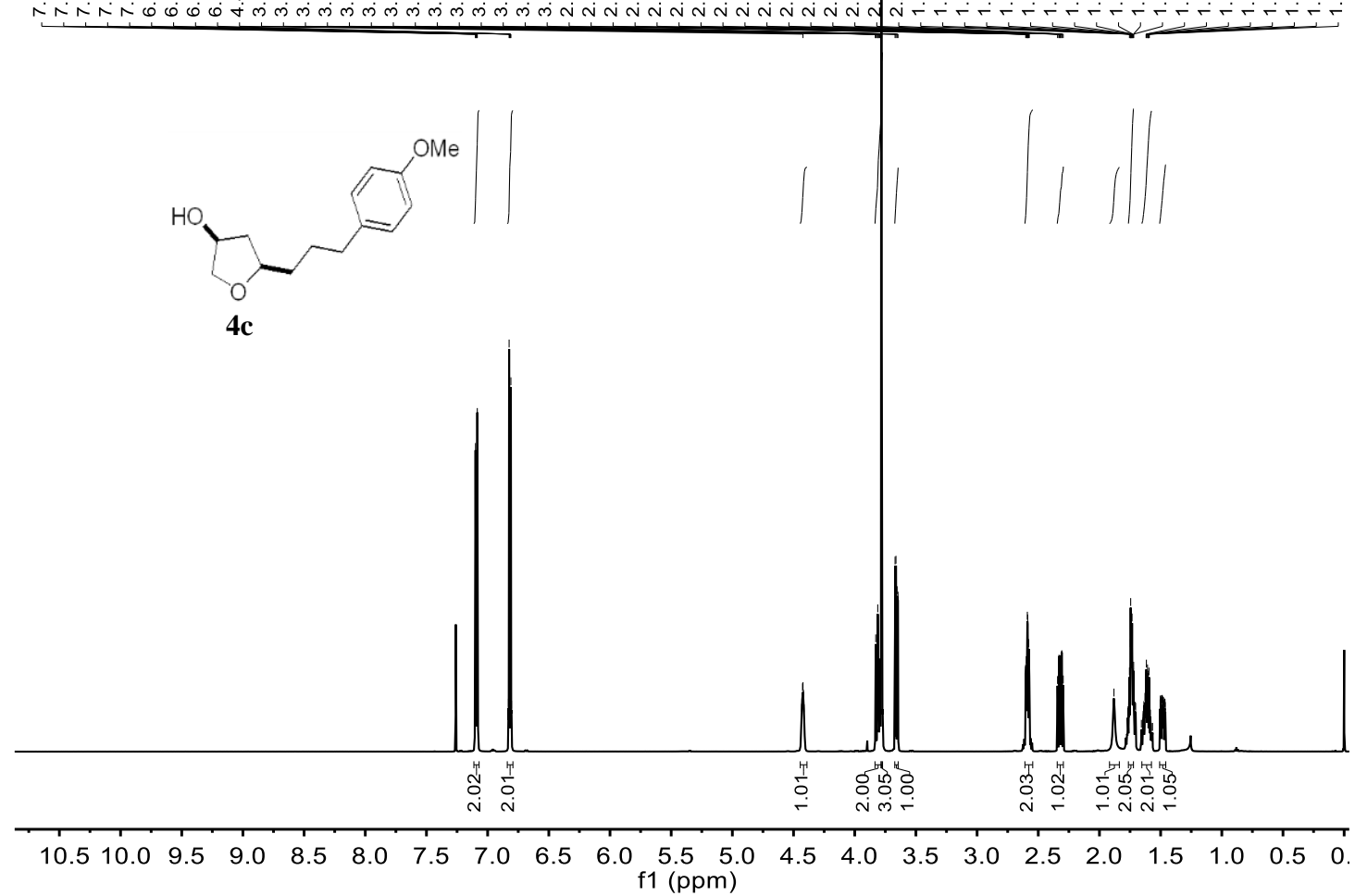




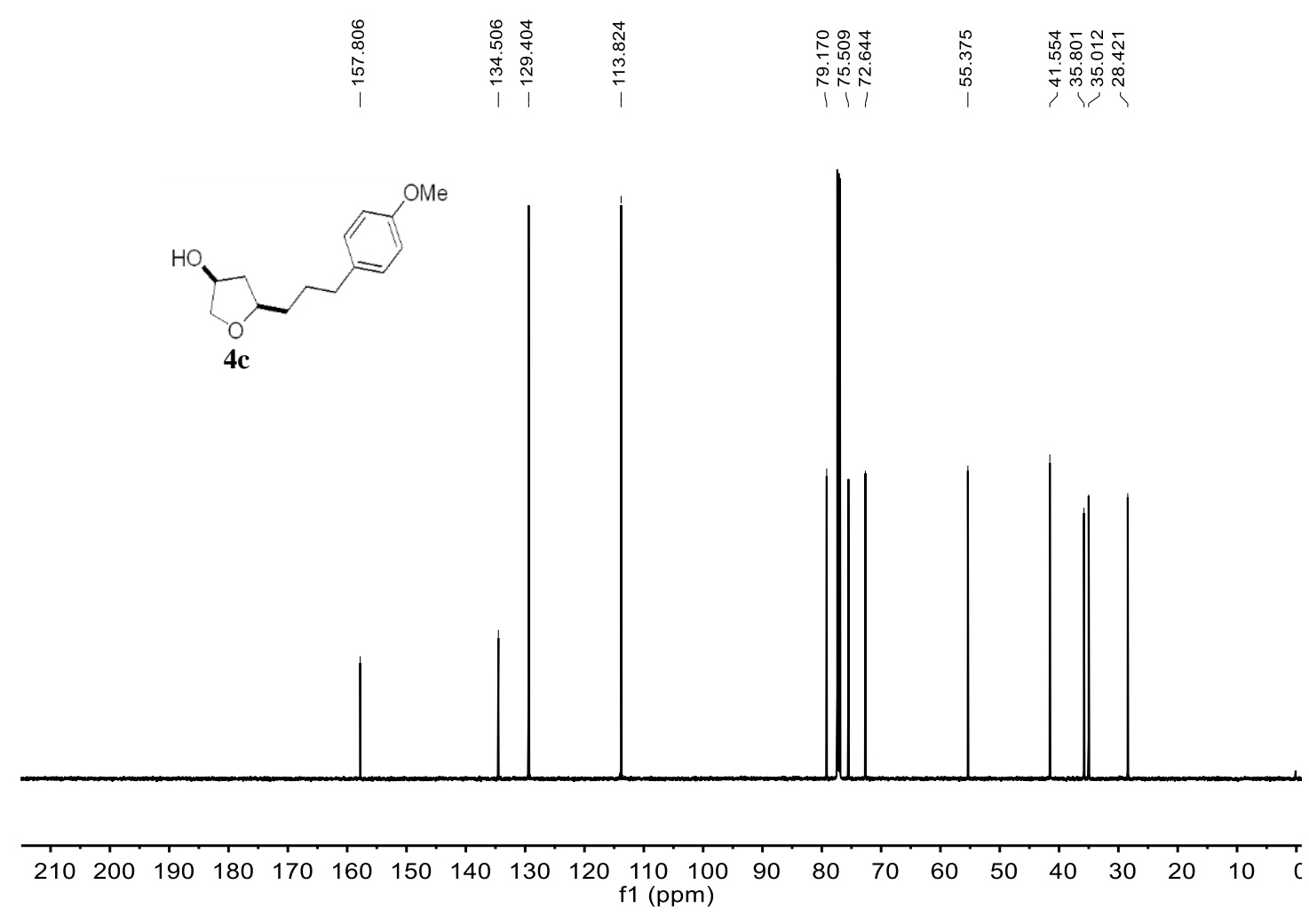

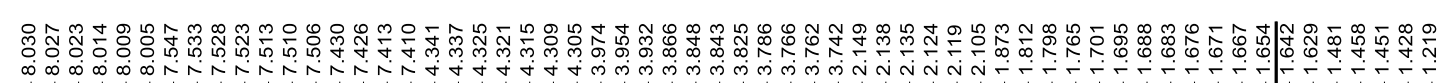

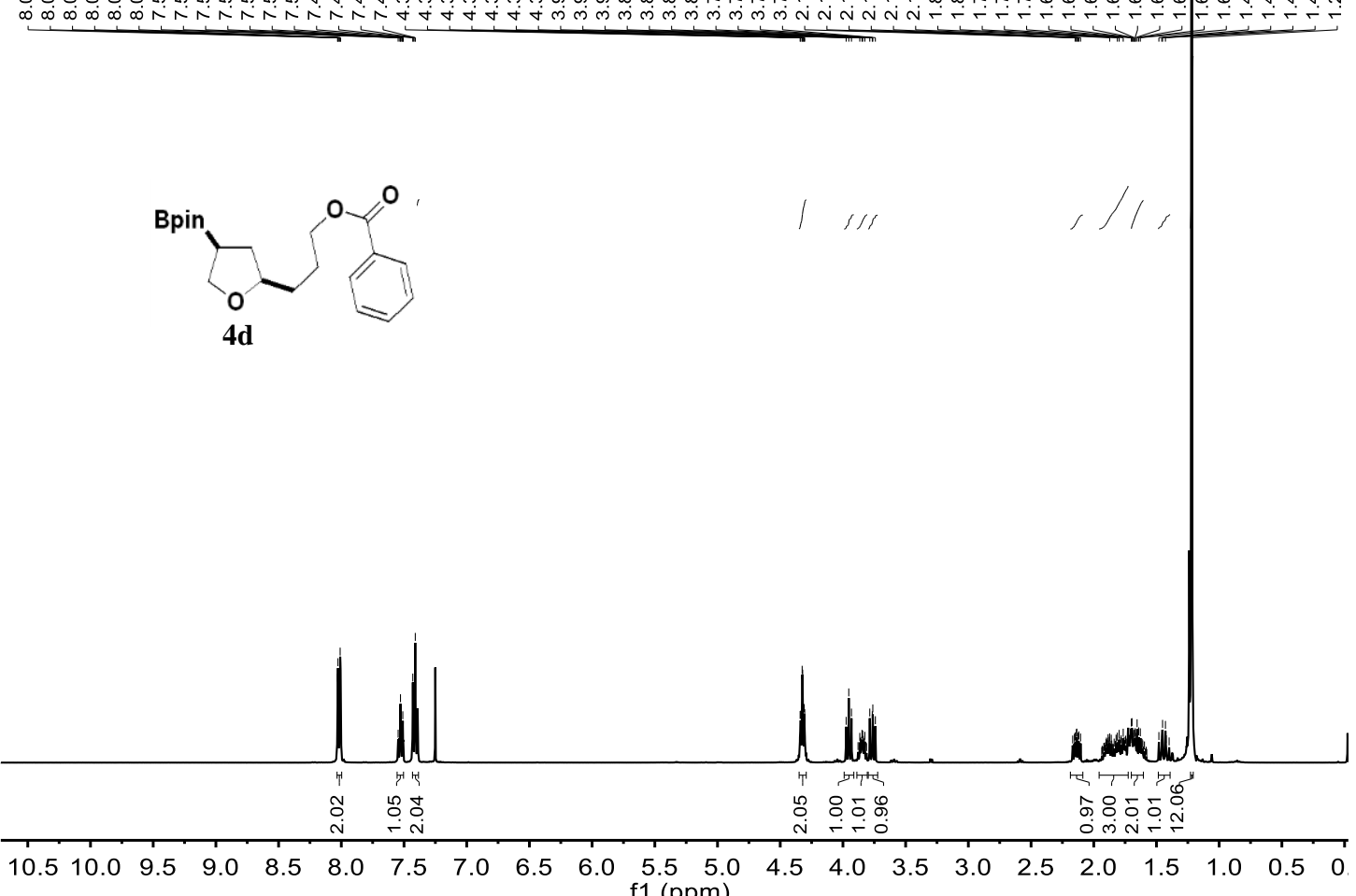



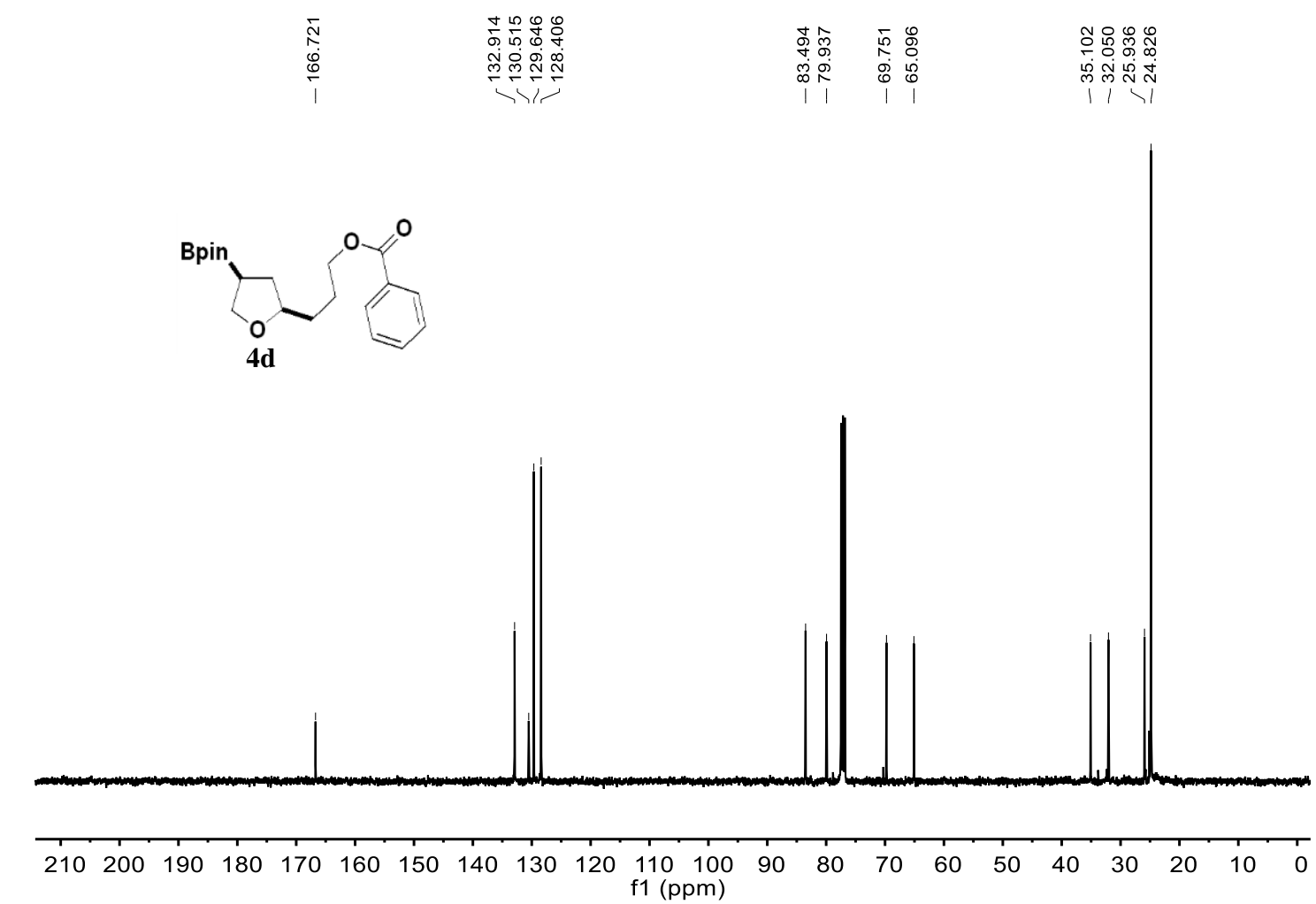

\section{\&
in
लm
|}
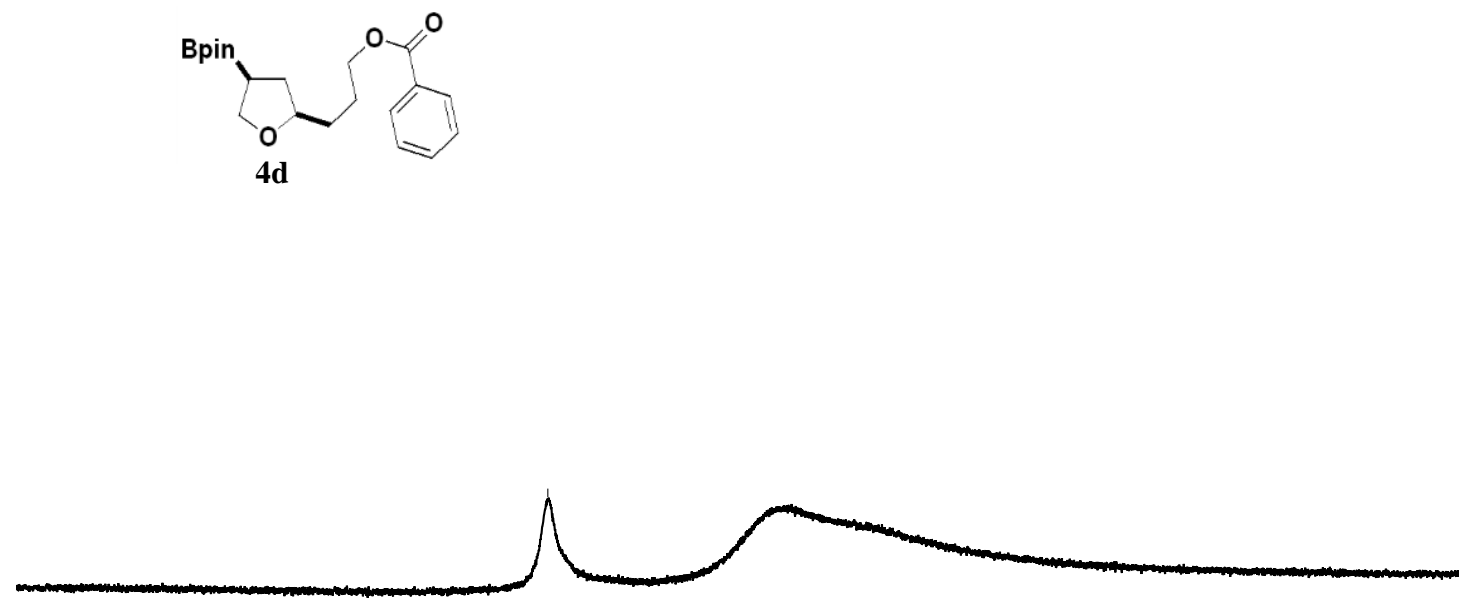

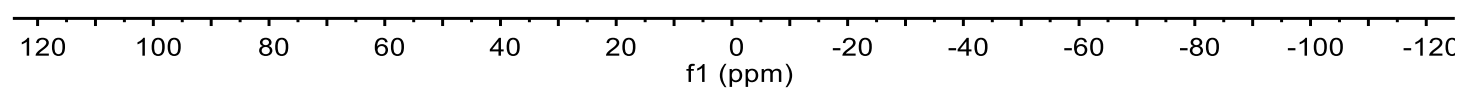



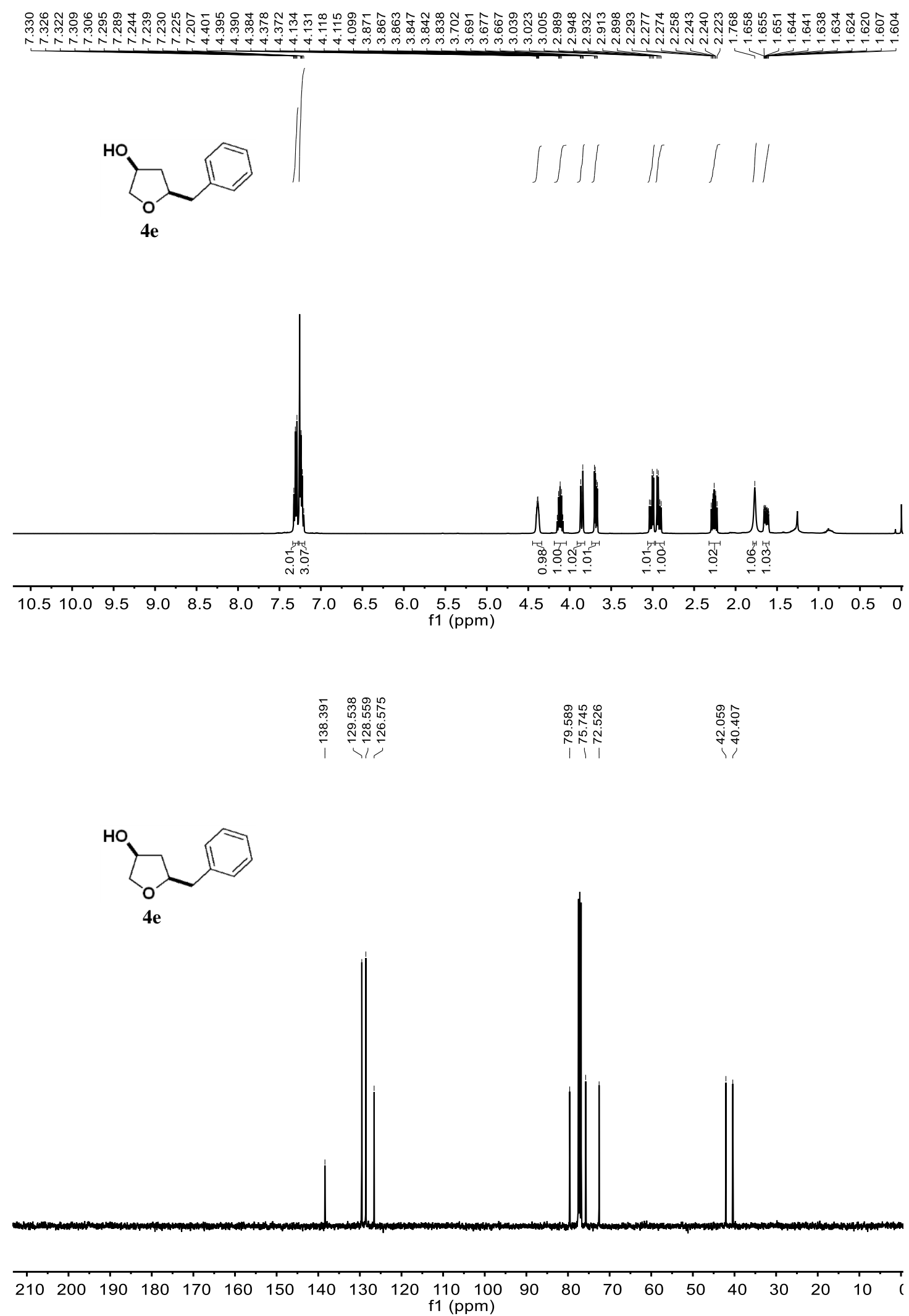


\section{HO}
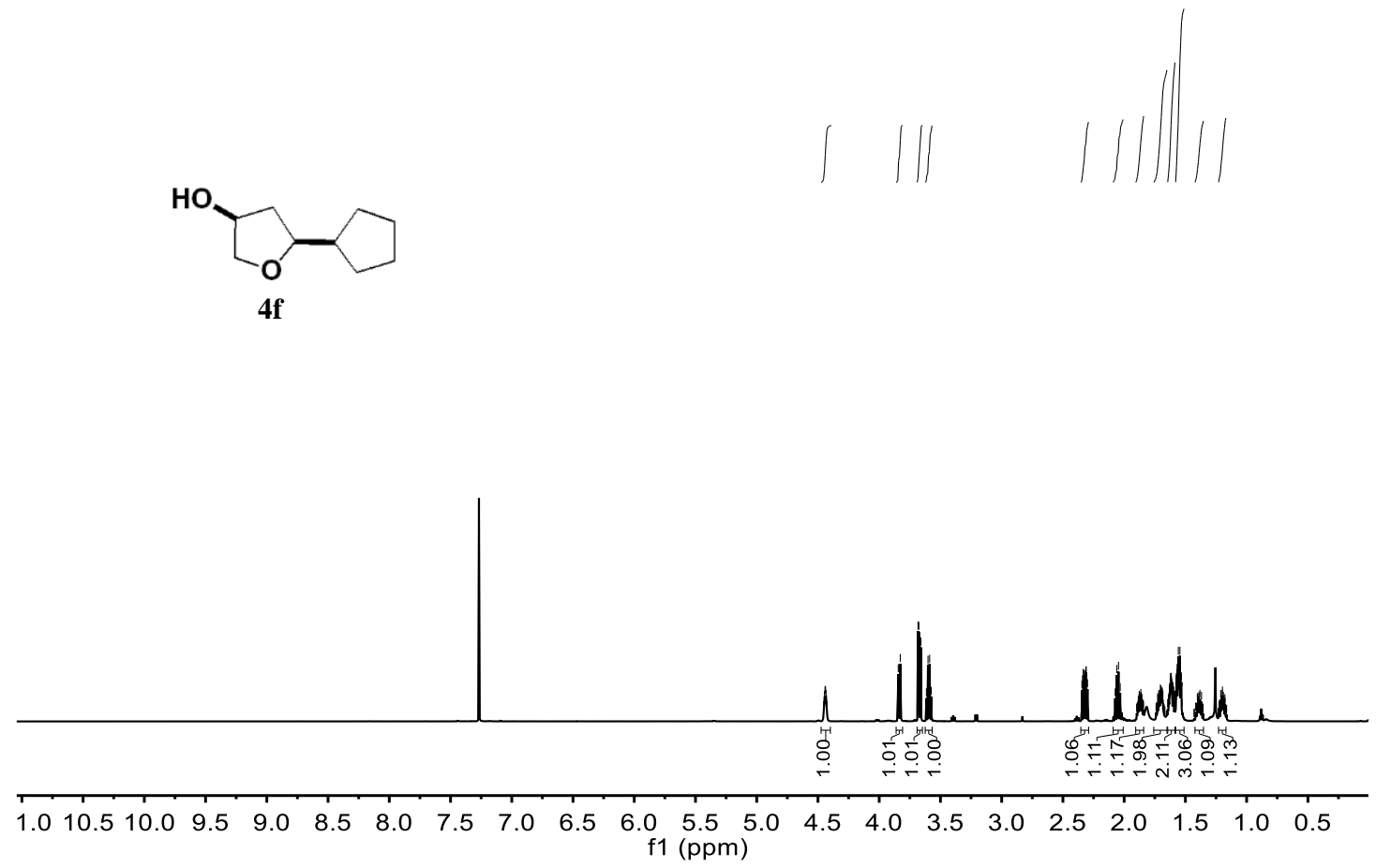

HO<smiles>IC1COC(C2CCCC2)C1</smiles>

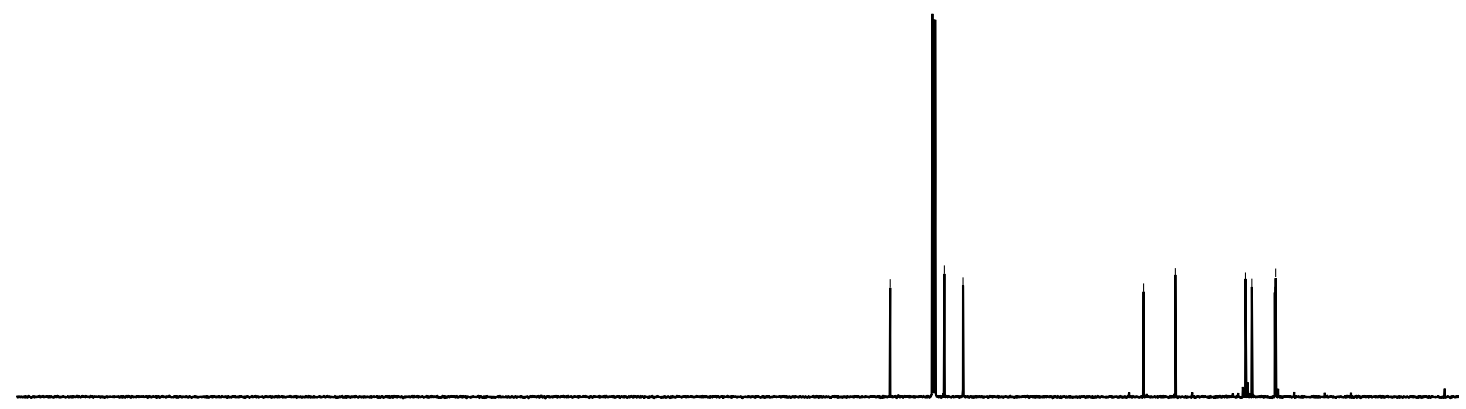

$\begin{array}{llllllllllllllllllllll}210 & 200 & 190 & 180 & 170 & 160 & 150 & 140 & 130 & 120 & 110 & 100 & 90 & 80 & 70 & 60 & 50 & 40 & 30 & 20 & 10 & 0\end{array}$ f1 (ppm) 

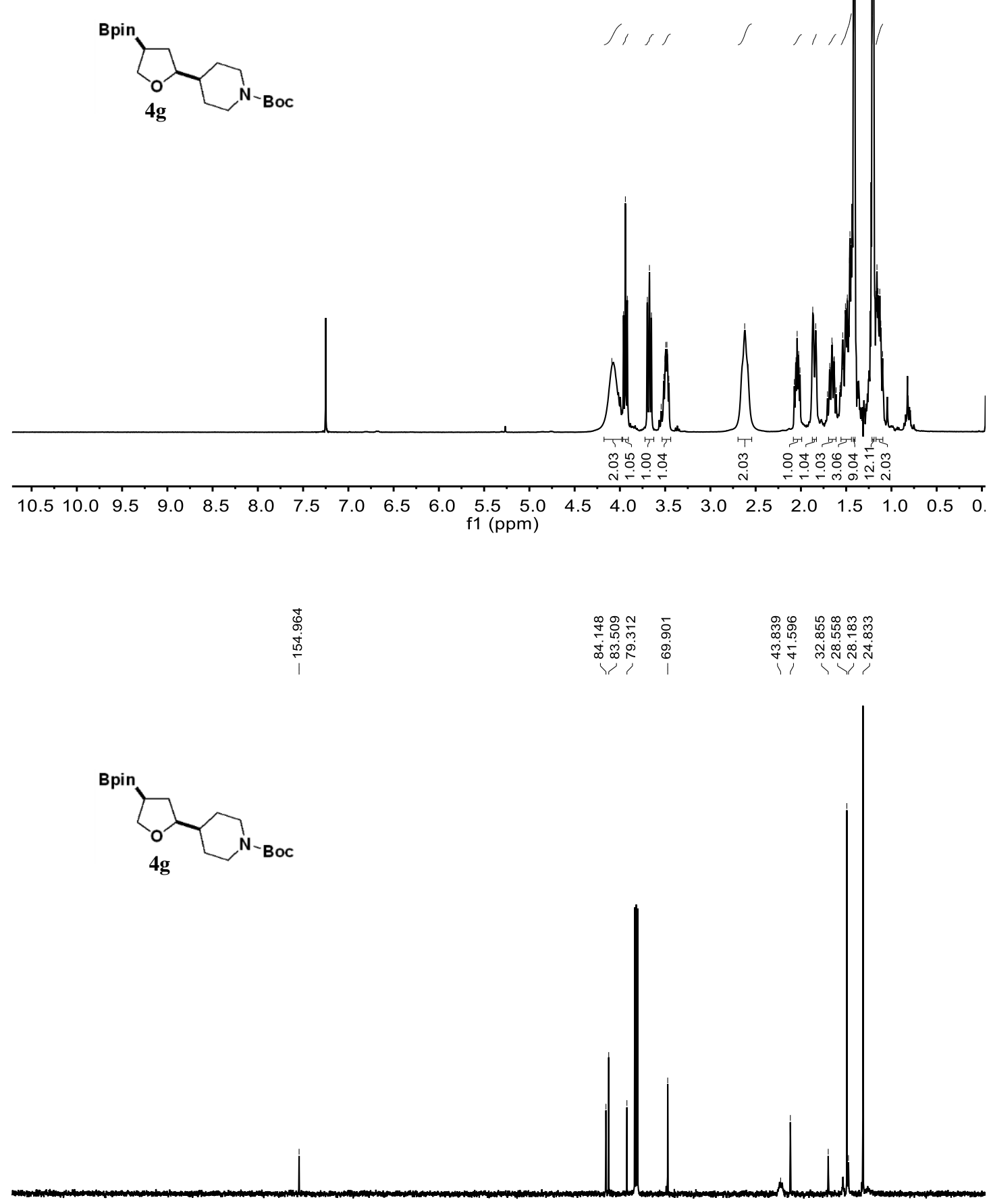

$4 \mathrm{~g}$

$\begin{array}{llllllllllllllllllllllll}20 & 210 & 200 & 190 & 180 & 170 & 160 & 150 & 140 & 130 & 120 & 110 & 100 & 90 & 80 & 70 & 60 & 50 & 40 & 30 & 20 & 10 & 0\end{array}$ f1 (ppm) 

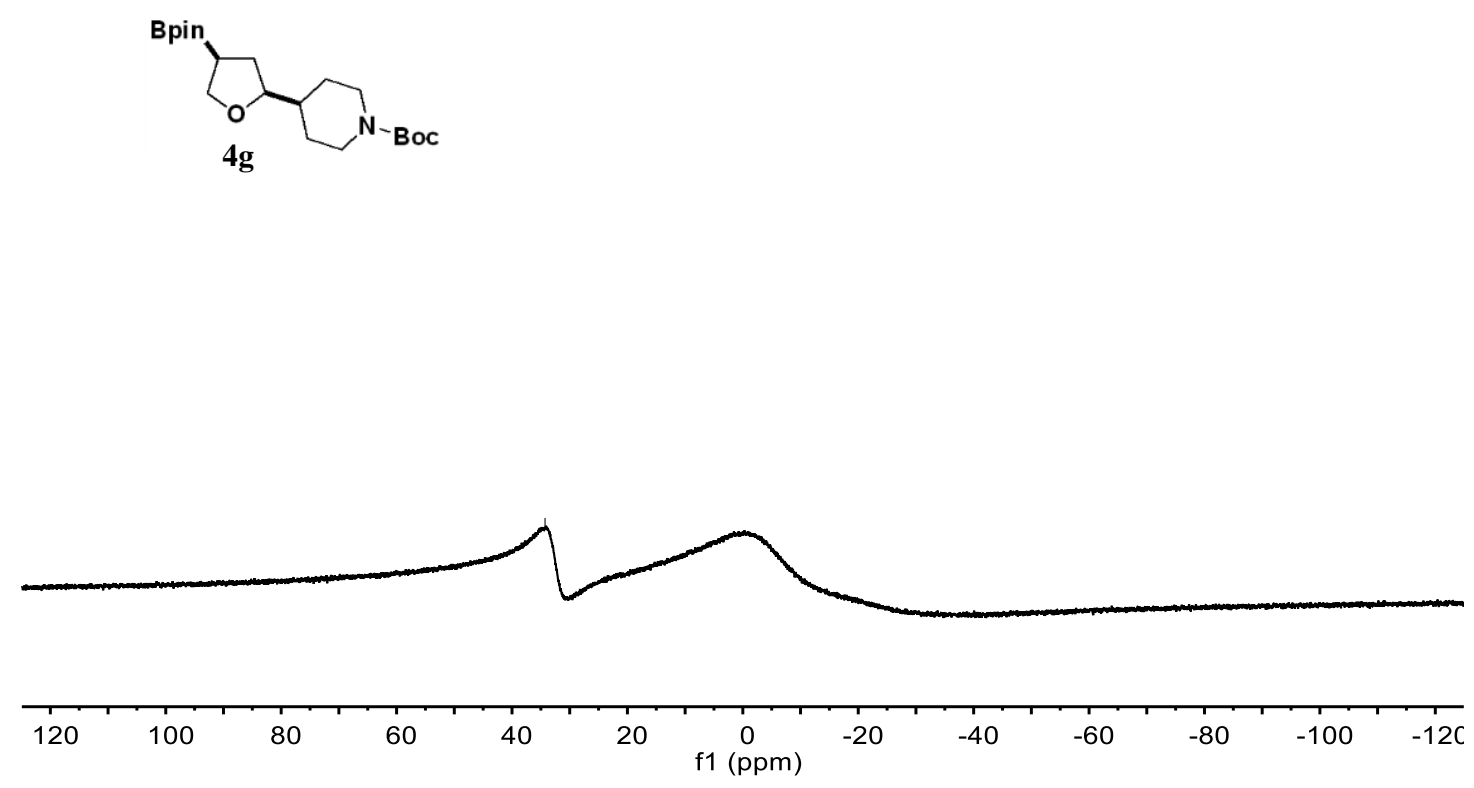

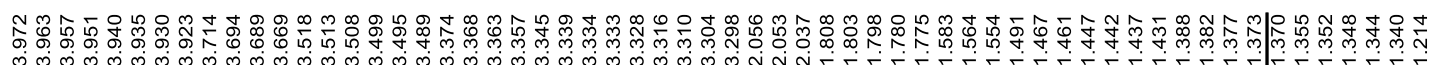

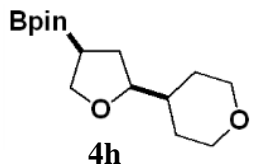

4h

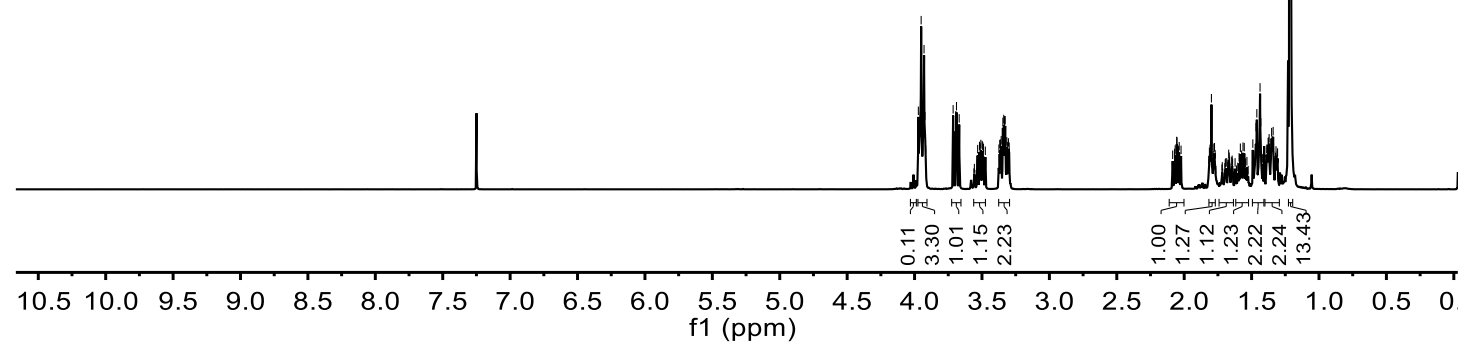


Bpin<smiles>[CH2+]OC1CCOCC1C1CCOCC1</smiles>

4h

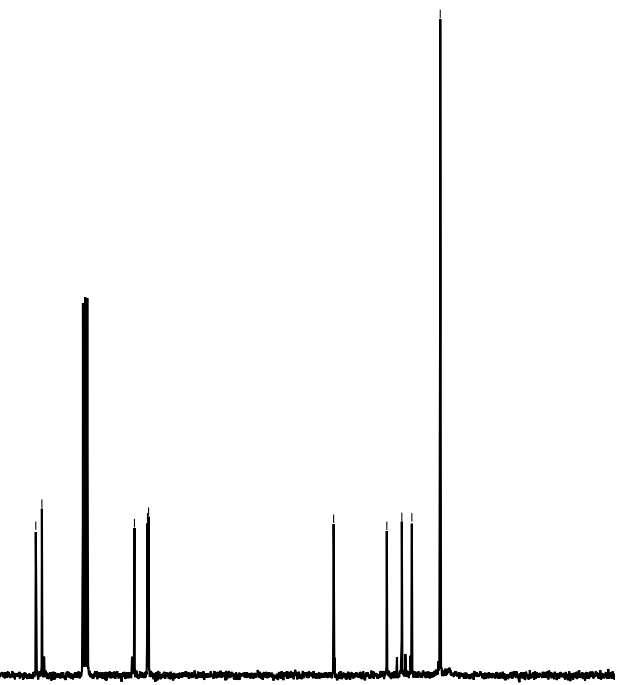

$\begin{array}{llllllllllllllllllllll}? 10 & 200 & 190 & 180 & 170 & 160 & 150 & 140 & 130 & 120 & 110 & 100 & 90 & 80 & 70 & 60 & 50 & 40 & 30 & 20 & 10 & \mathrm{C}\end{array}$ f1 (ppm)
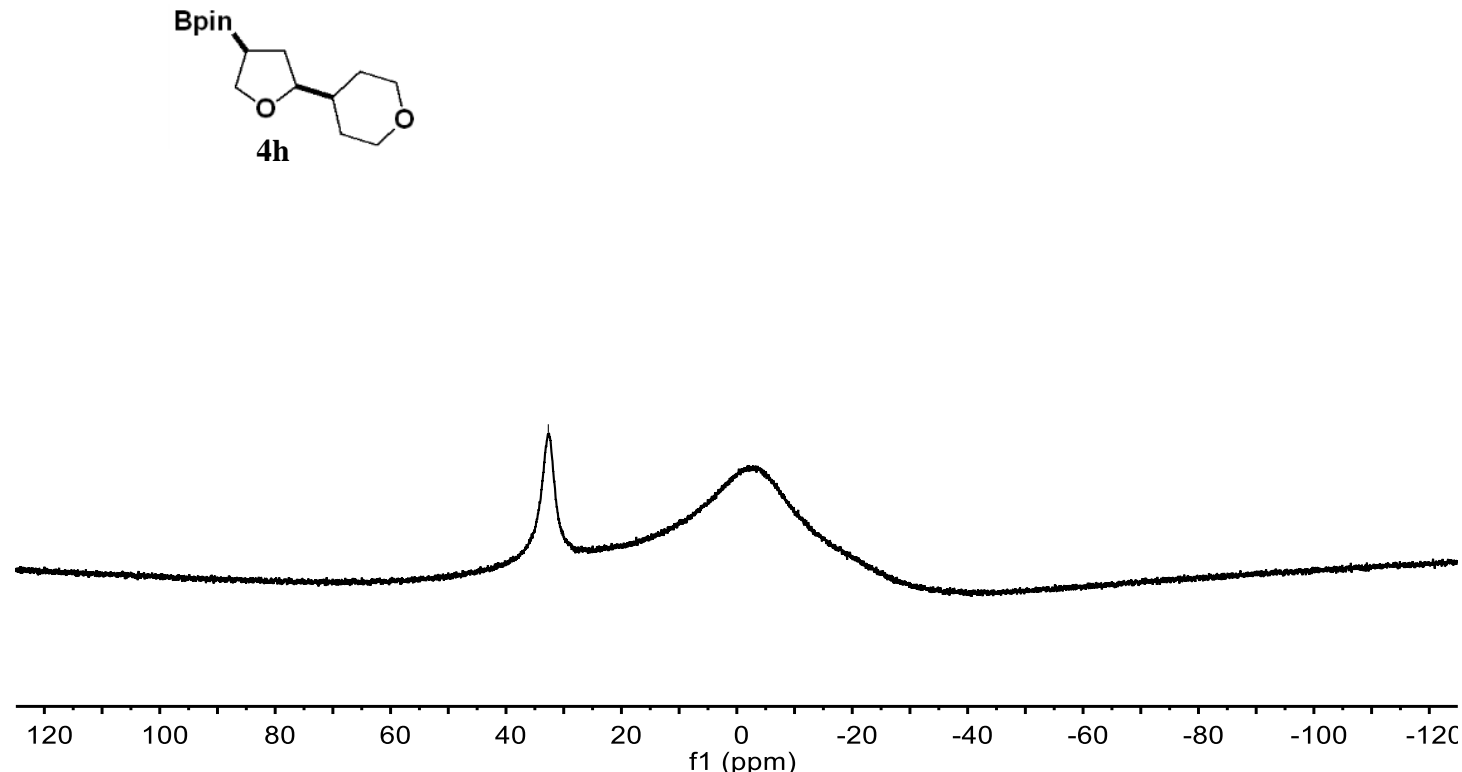

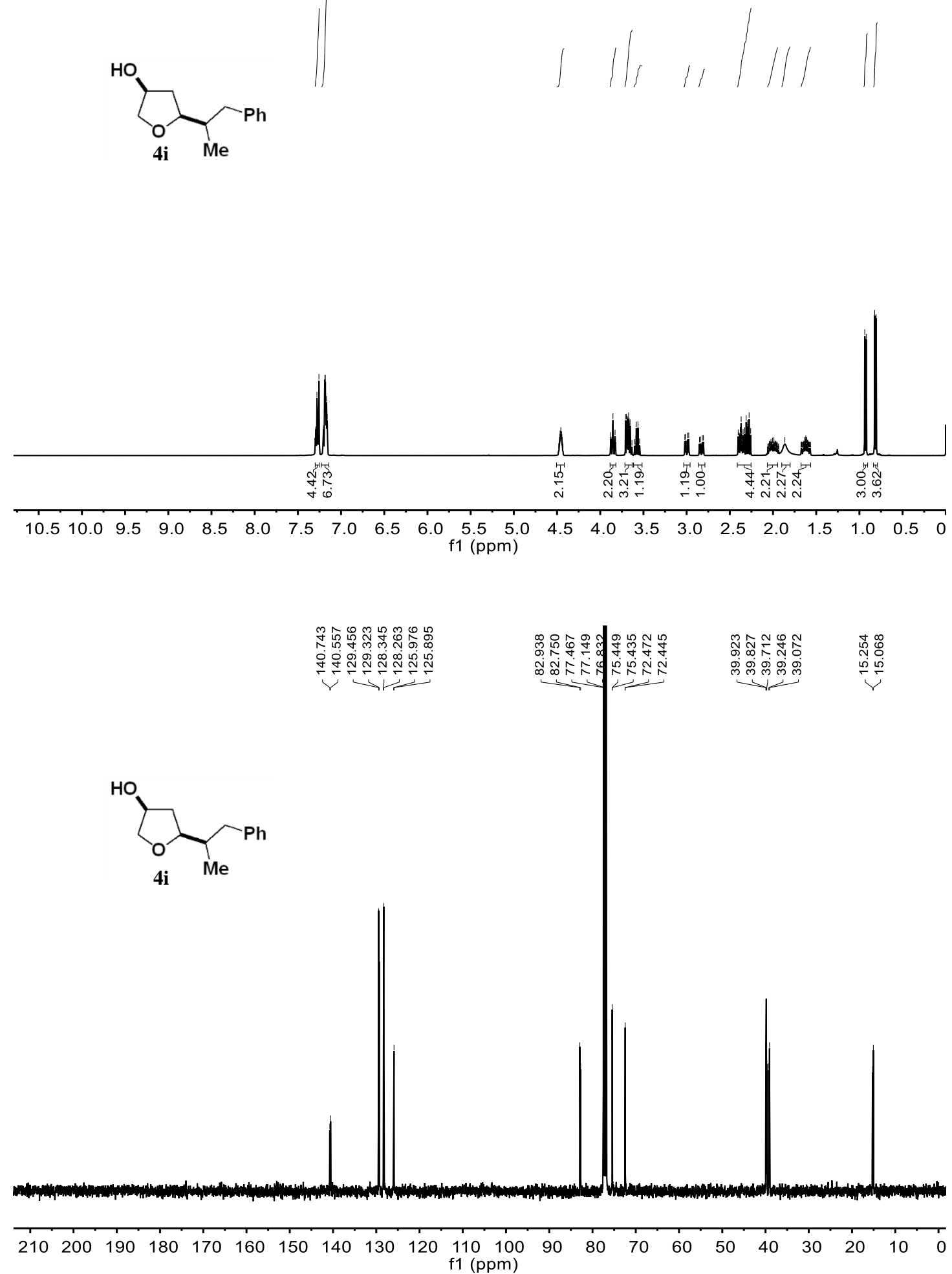


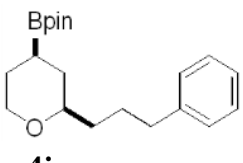

$4 j$
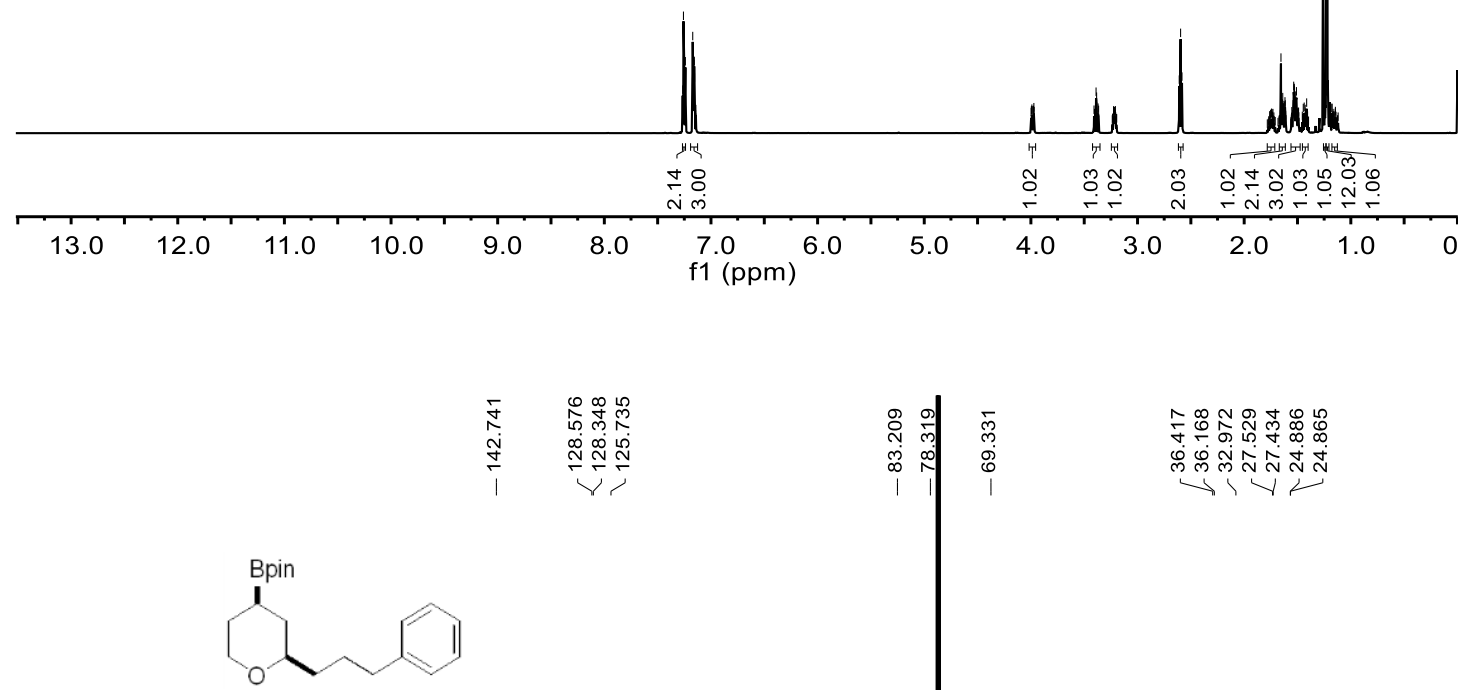

$4 \mathbf{j}$

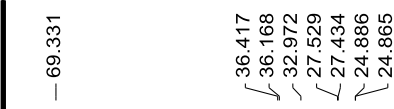

$\begin{array}{lllllllllllllllllllll}210 & 200 & 190 & 180 & 170 & 160 & 150 & 140 & 130 & 120 & 110 & 100 & 90 & 80 & 70 & 60 & 50 & 40 & 30 & 20 & 10\end{array}$ f1 $(\mathrm{ppm})$ 

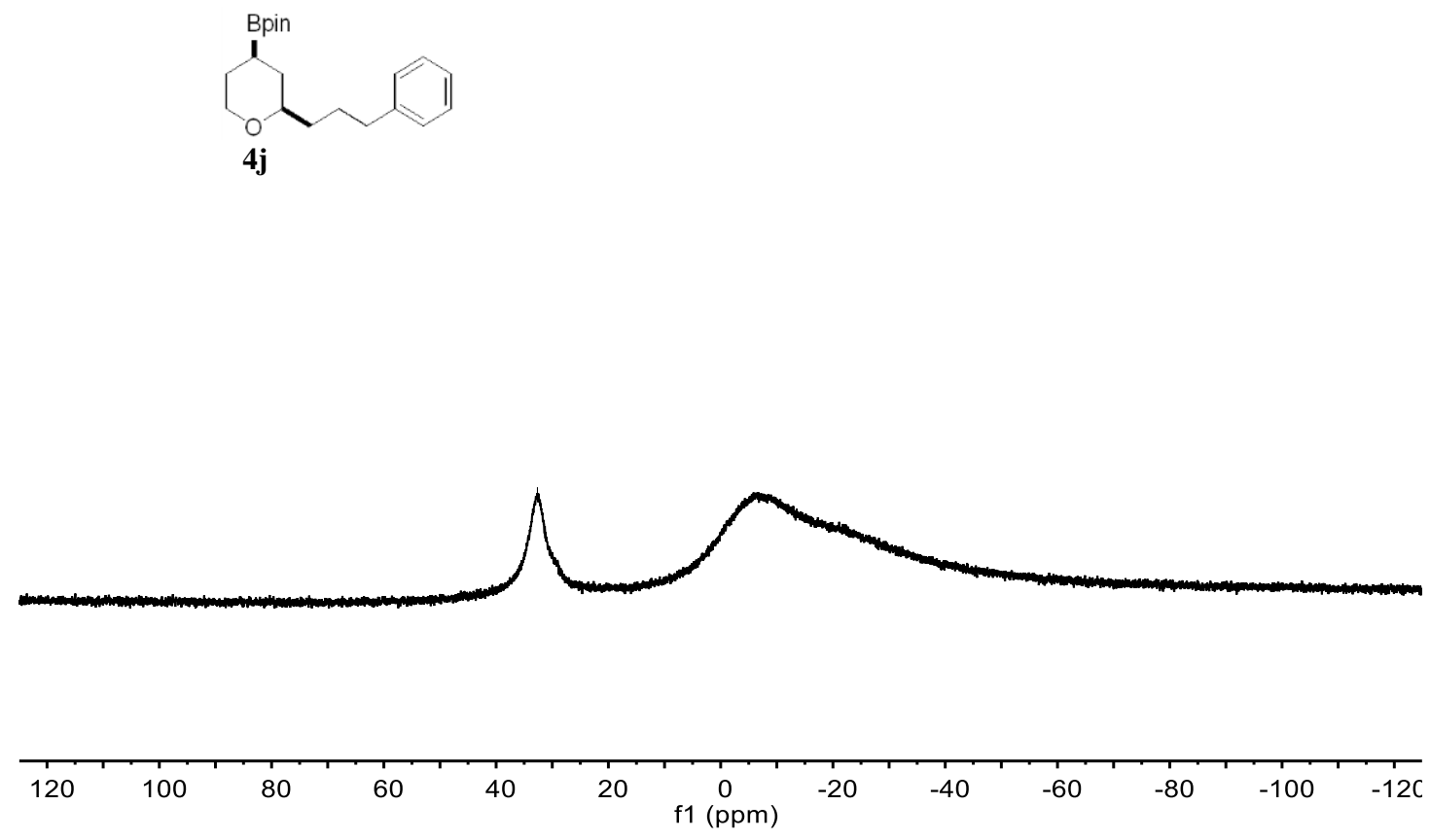

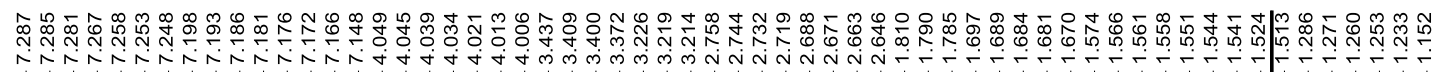
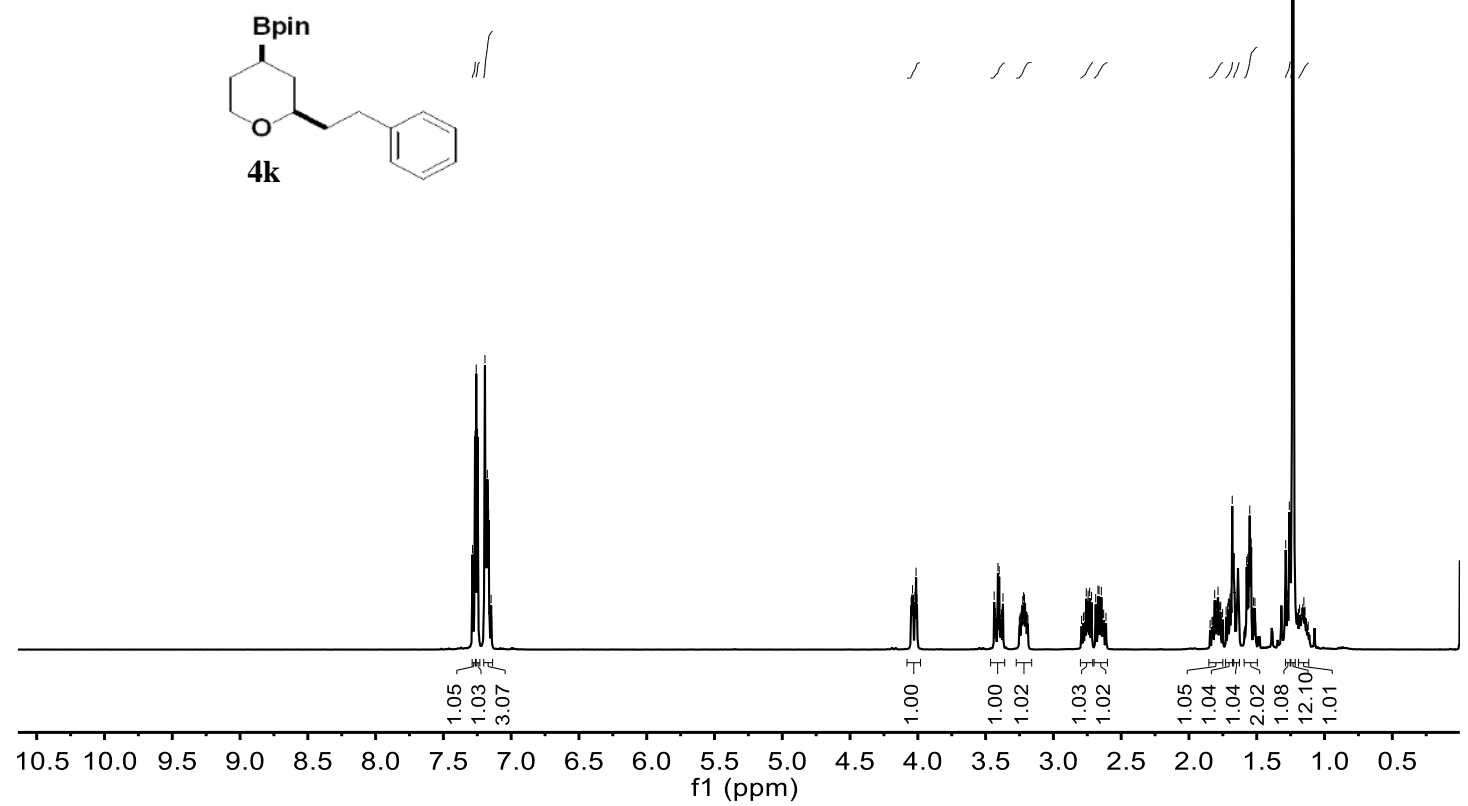


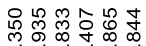

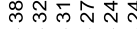

Bpin

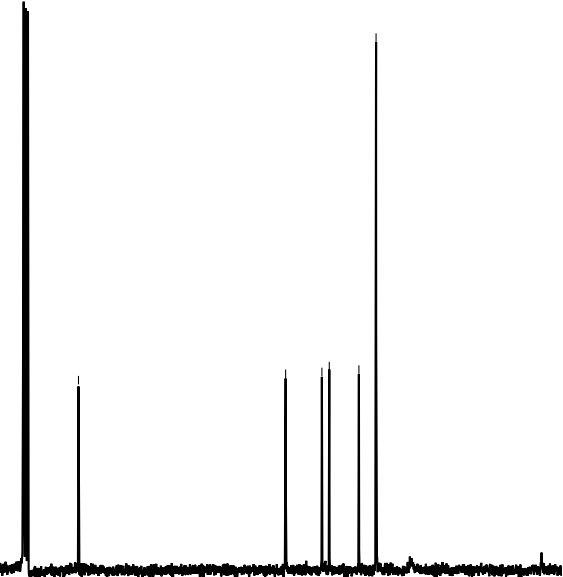

$\begin{array}{llllllllllllllllllllll}210 & 200 & 190 & 180 & 170 & 160 & 150 & 140 & 130 & 120 & 110 & 100 & 90 & 80 & 70 & 60 & 50 & 40 & 30 & 20 & 10 & 0\end{array}$ f1 (ppm)
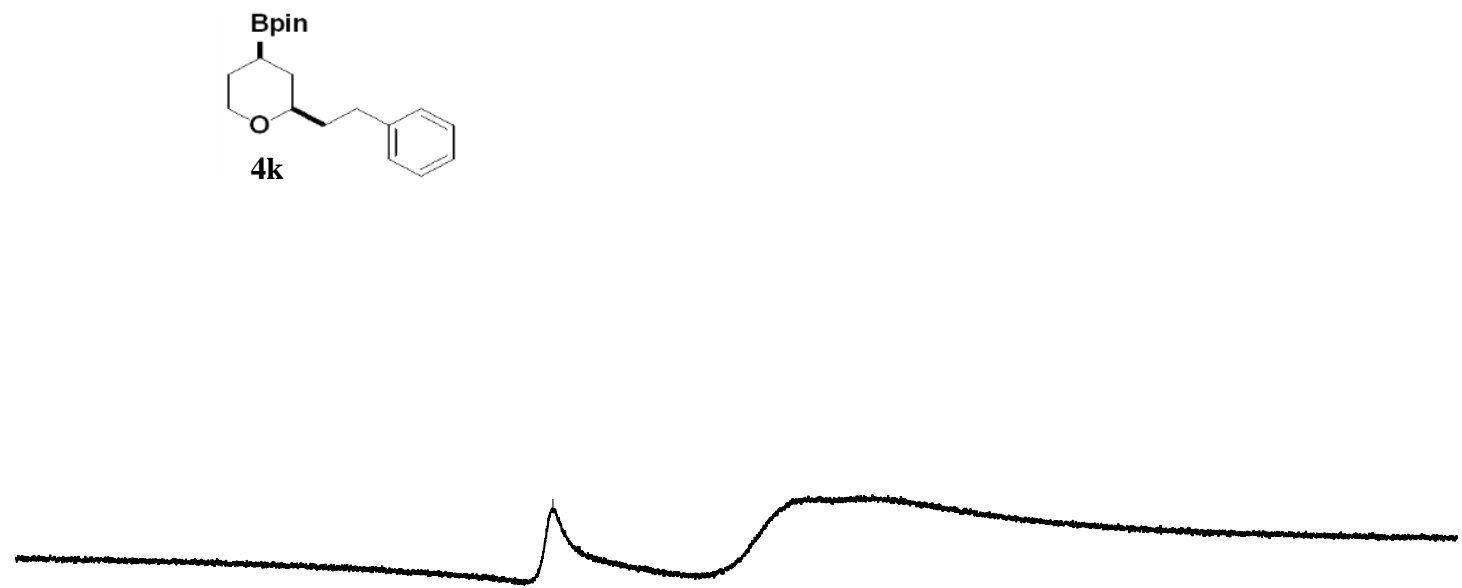

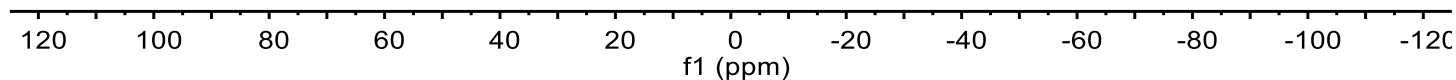


<smiles>CC(CCC1C[C@H]([SnH3])CCO1)(c1ccccc1)c1ccccc1</smiles>

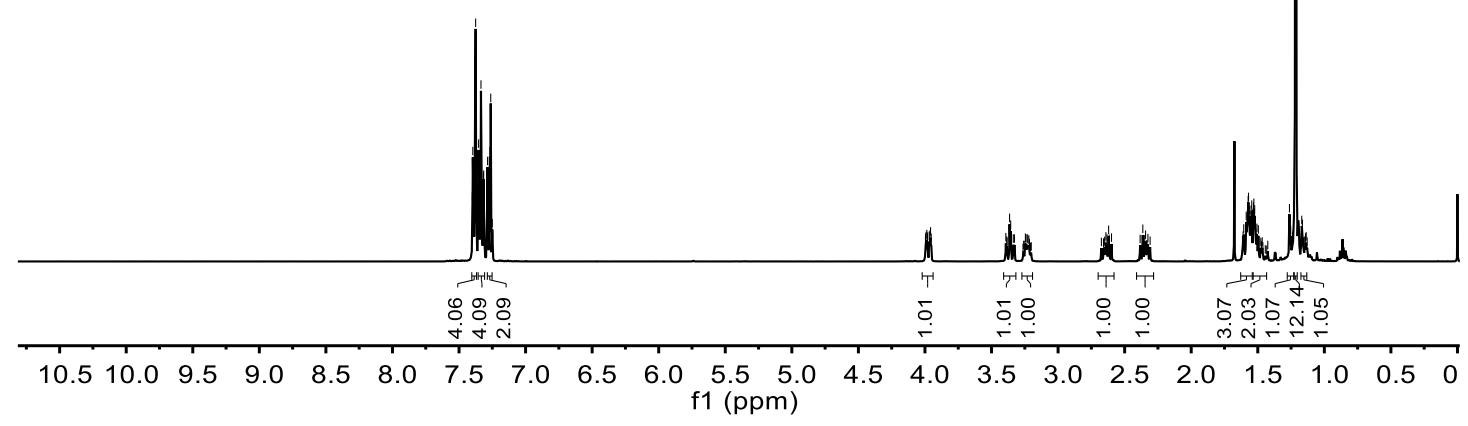

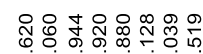

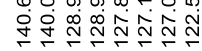

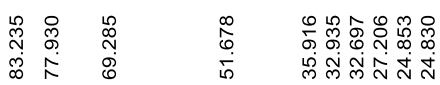

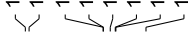

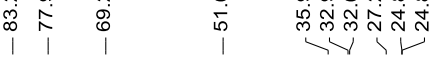

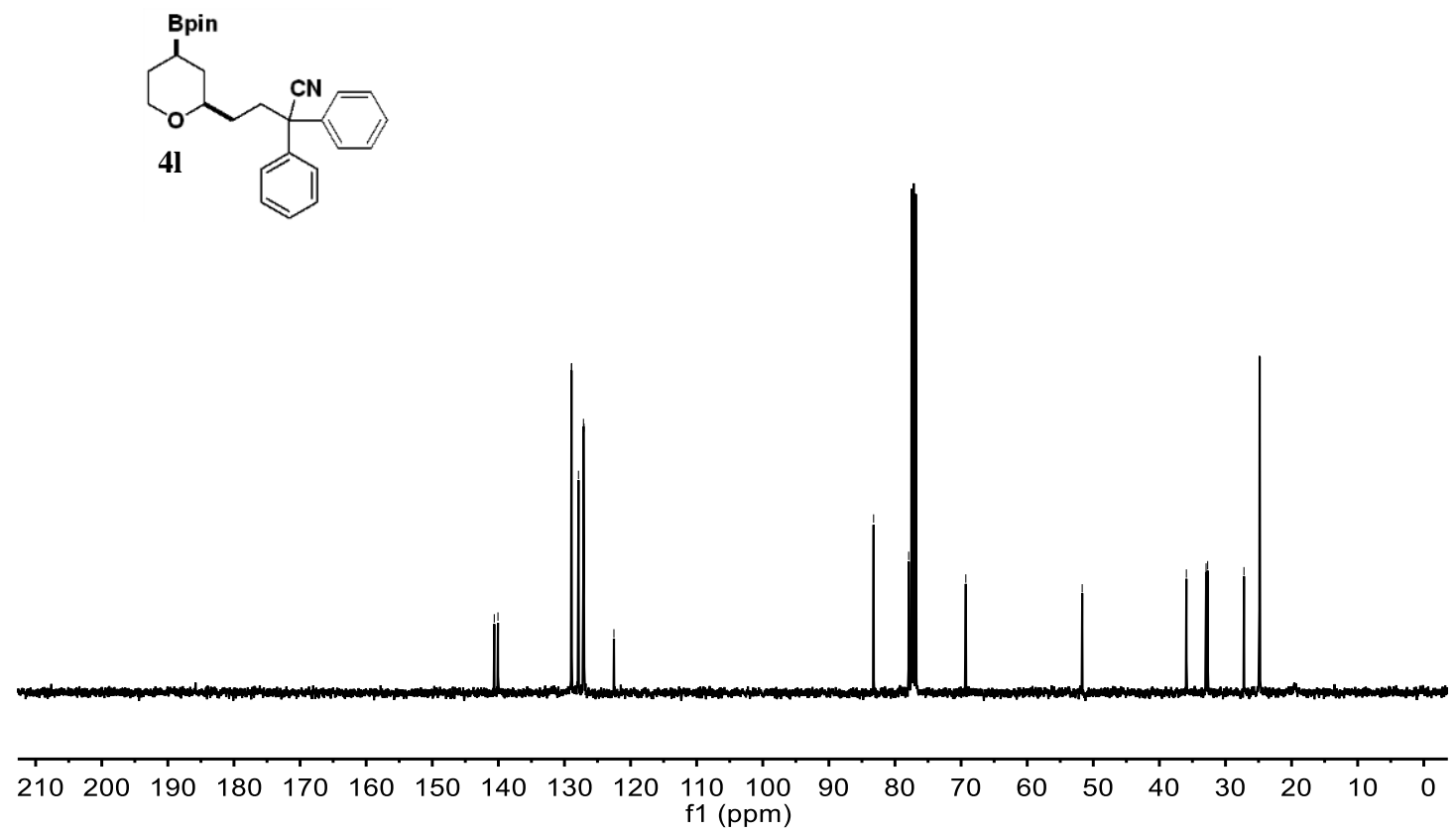



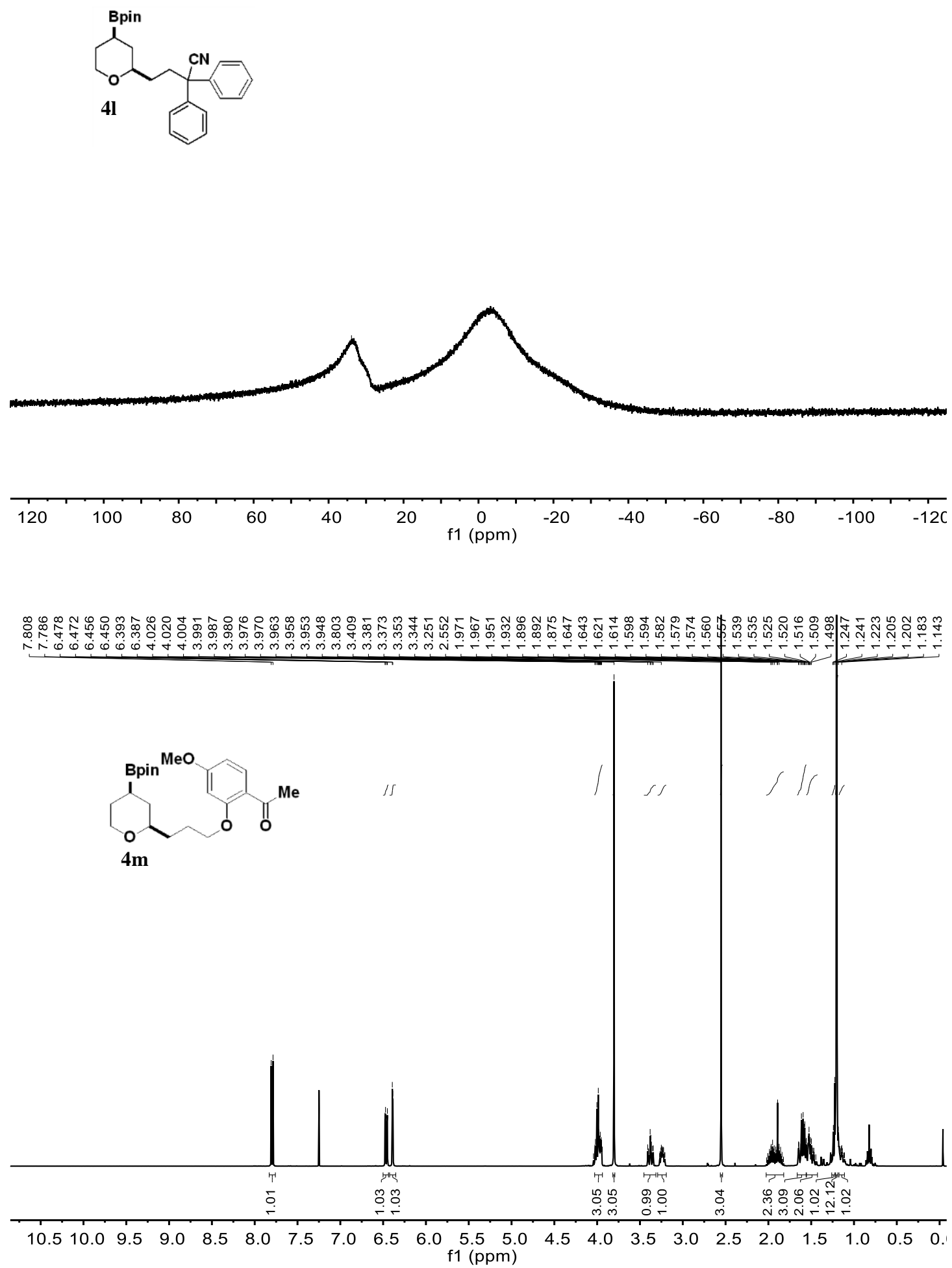


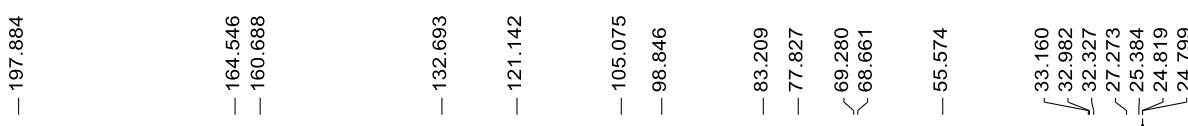<smiles>COc1ccc(C(C)=O)c(OCCCC2CC([SnH3])CCO2)c1</smiles>

$4 m$

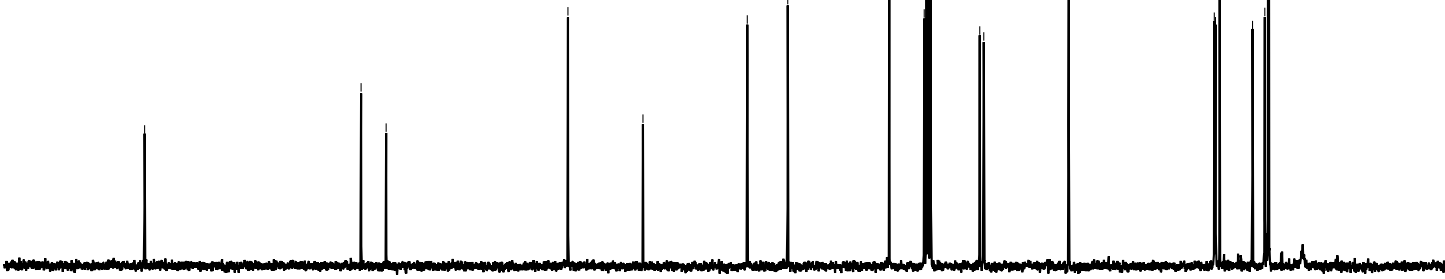

$\begin{array}{llllllllllllllllllllll}210 & 200 & 190 & 180 & 170 & 160 & 150 & 140 & 130 & 120 & 110 & 100 & 90 & 80 & 70 & 60 & 50 & 40 & 30 & 20 & 10 & 0\end{array}$ f1 (ppm)

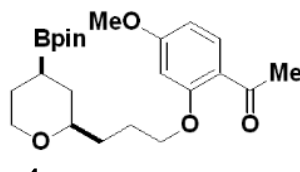

$4 \mathrm{~m}$
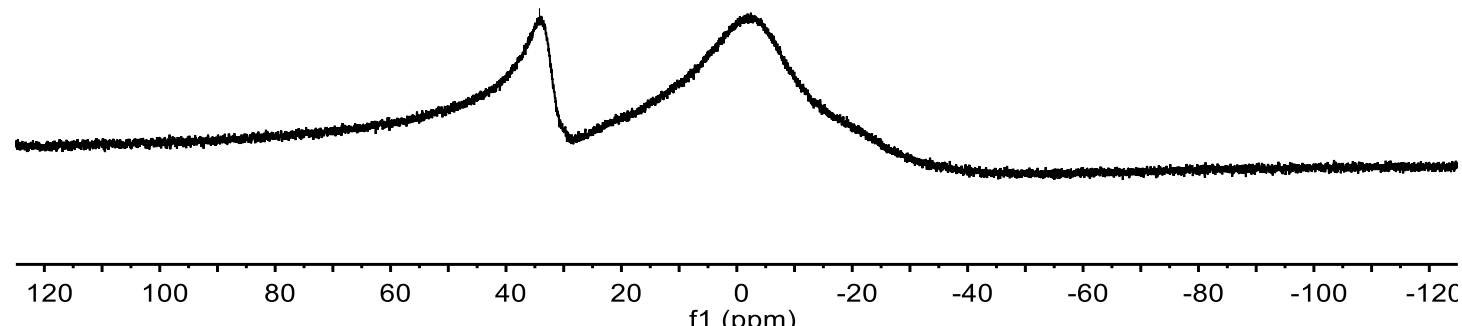


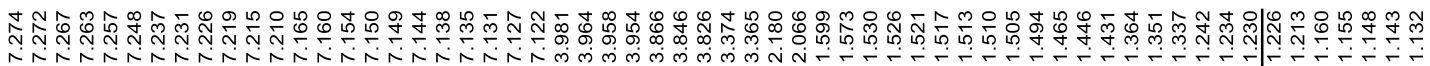<smiles>C#CC1CC(Cc2ccccc2)CCC1CCC(c1ccccc1)c1ccccc1</smiles>

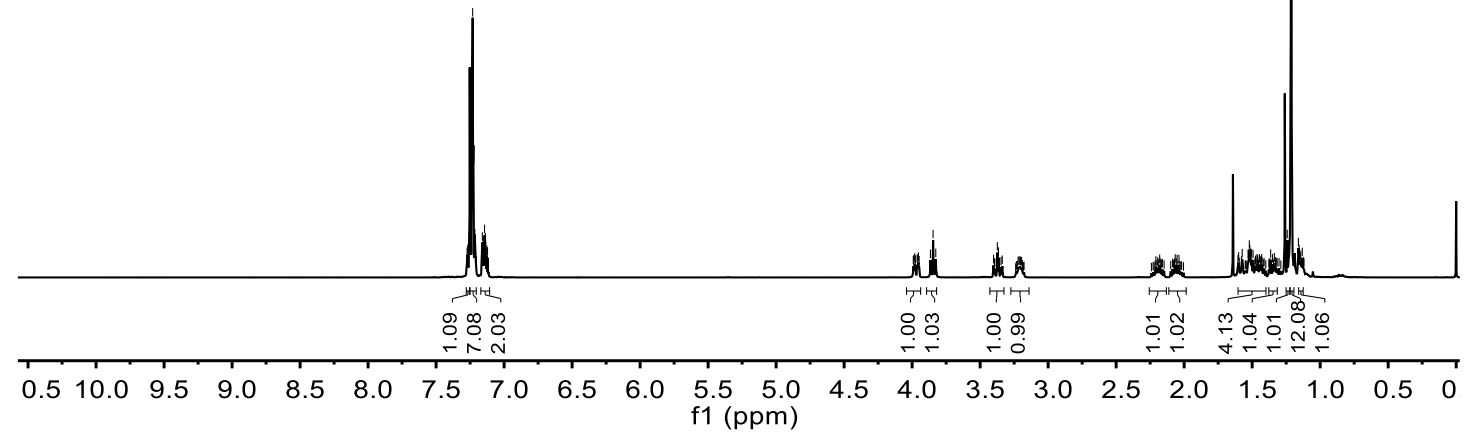<smiles>c1ccc(CCCC(c2ccccc2)c2ccccc2)cc1</smiles>

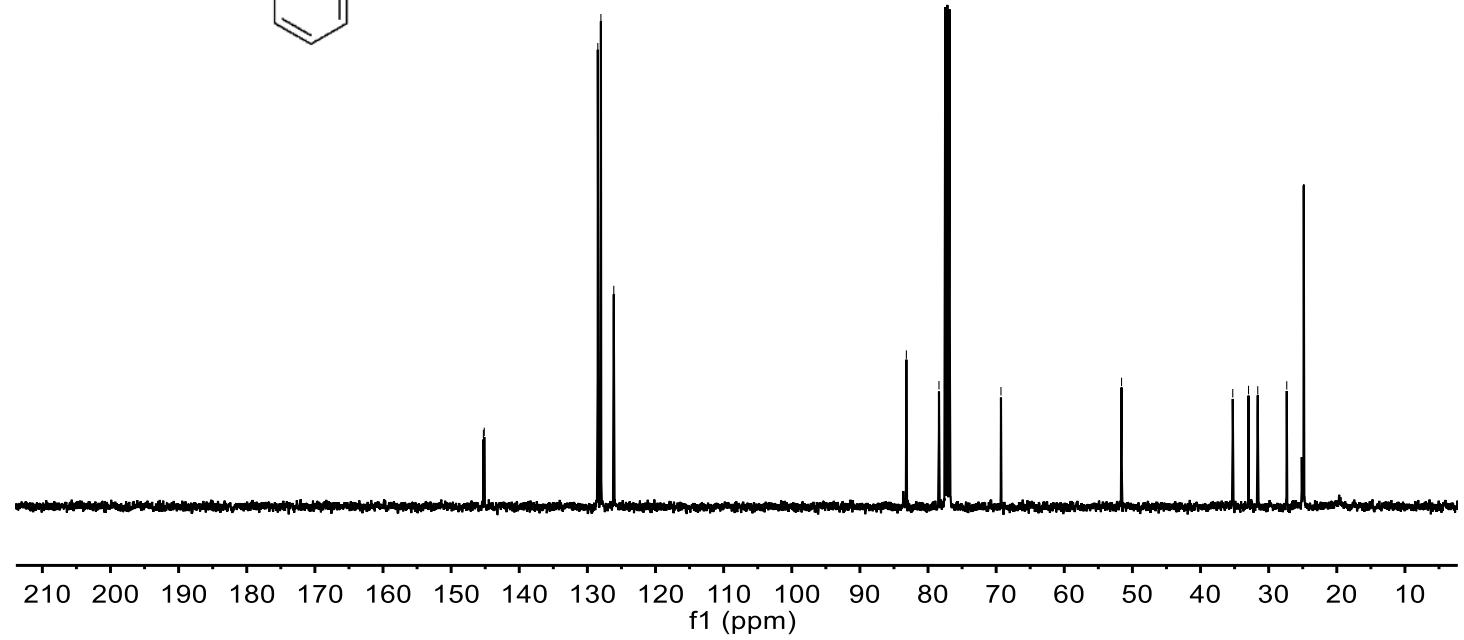


<smiles>BrC1CCOC(CCC(c2ccccc2)c2ccccc2)C1</smiles>

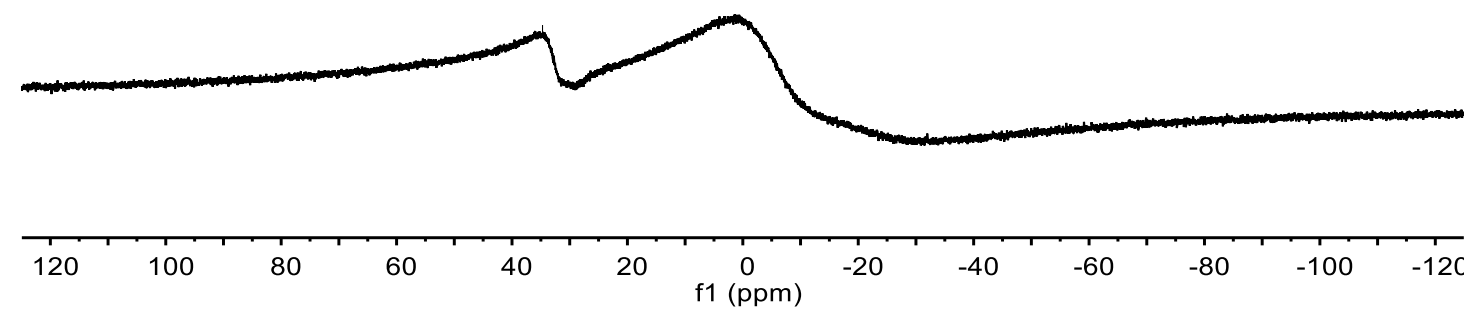

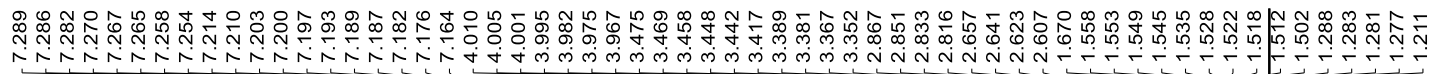

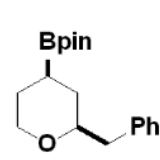

40

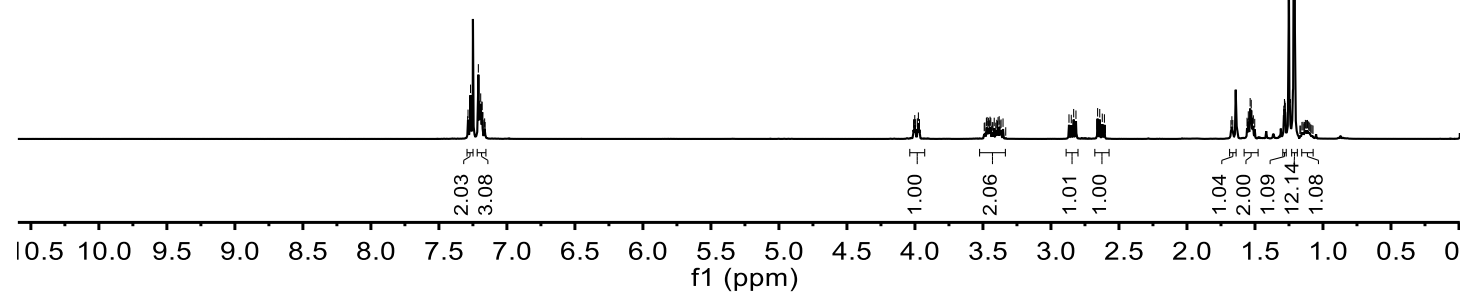




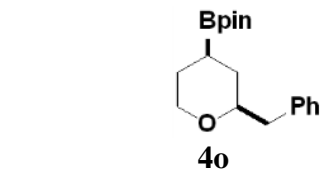

40

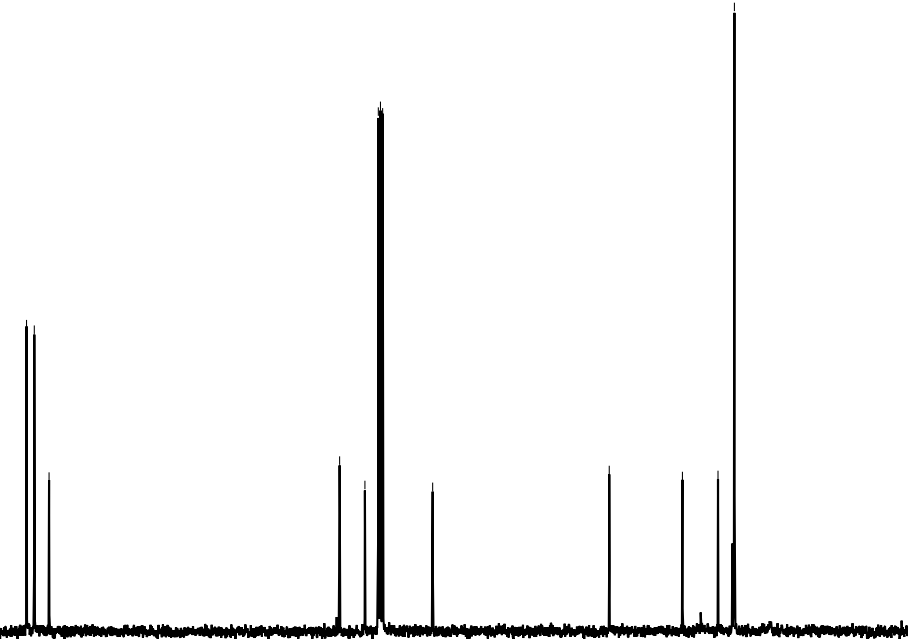

$\begin{array}{llllllllllllllllllllll}210 & 200 & 190 & 180 & 170 & 160 & 150 & 140 & 130 & 120 & 110 & 100 & 90 & 80 & 70 & 60 & 50 & 40 & 30 & 20 & 10 & c\end{array}$ $\mathrm{f1}(\mathrm{ppm})$

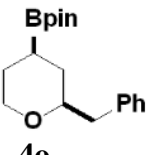

4o
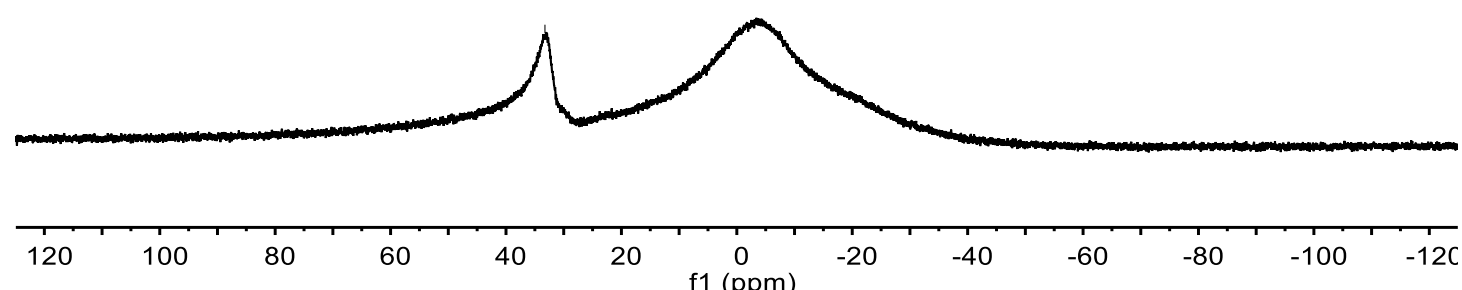


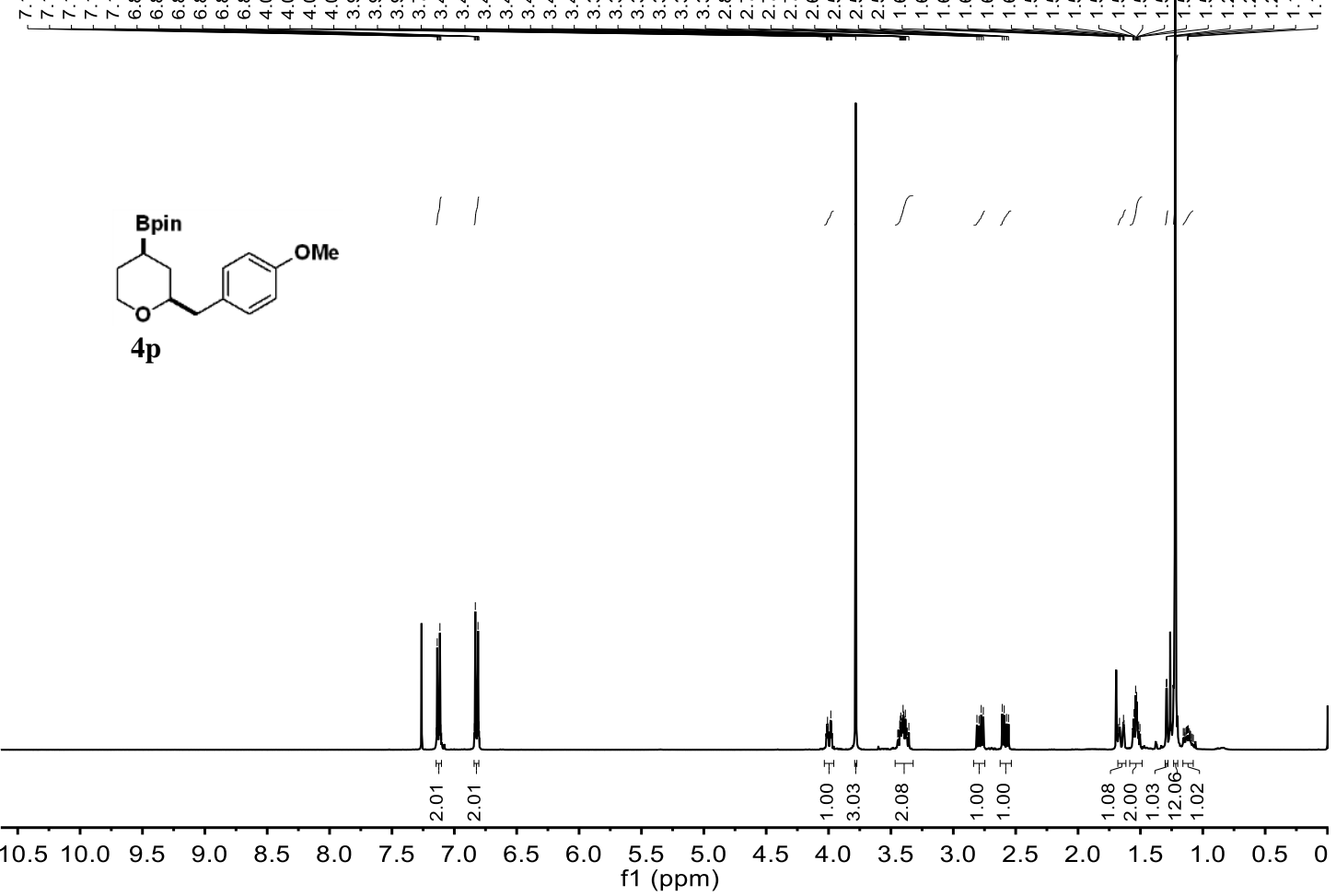

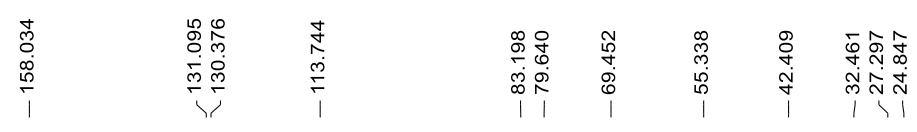
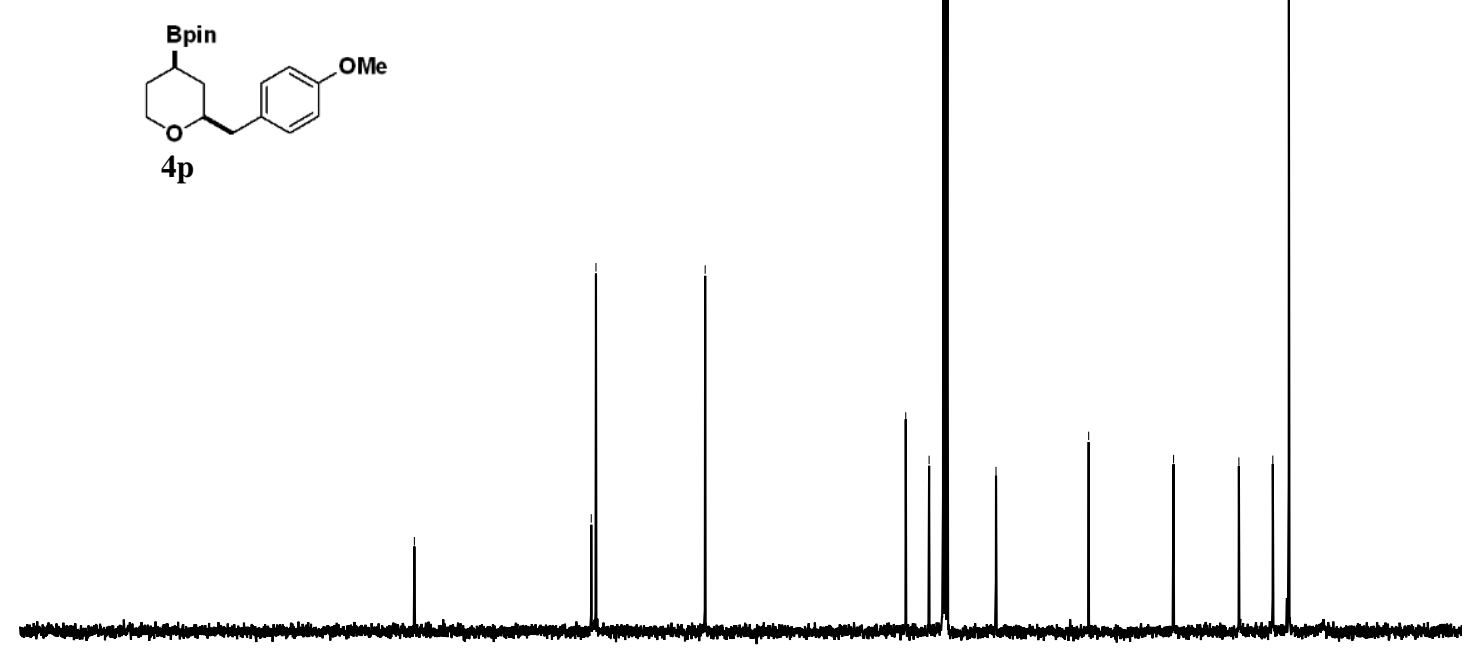

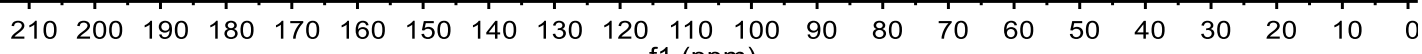
f1 (ppm) 

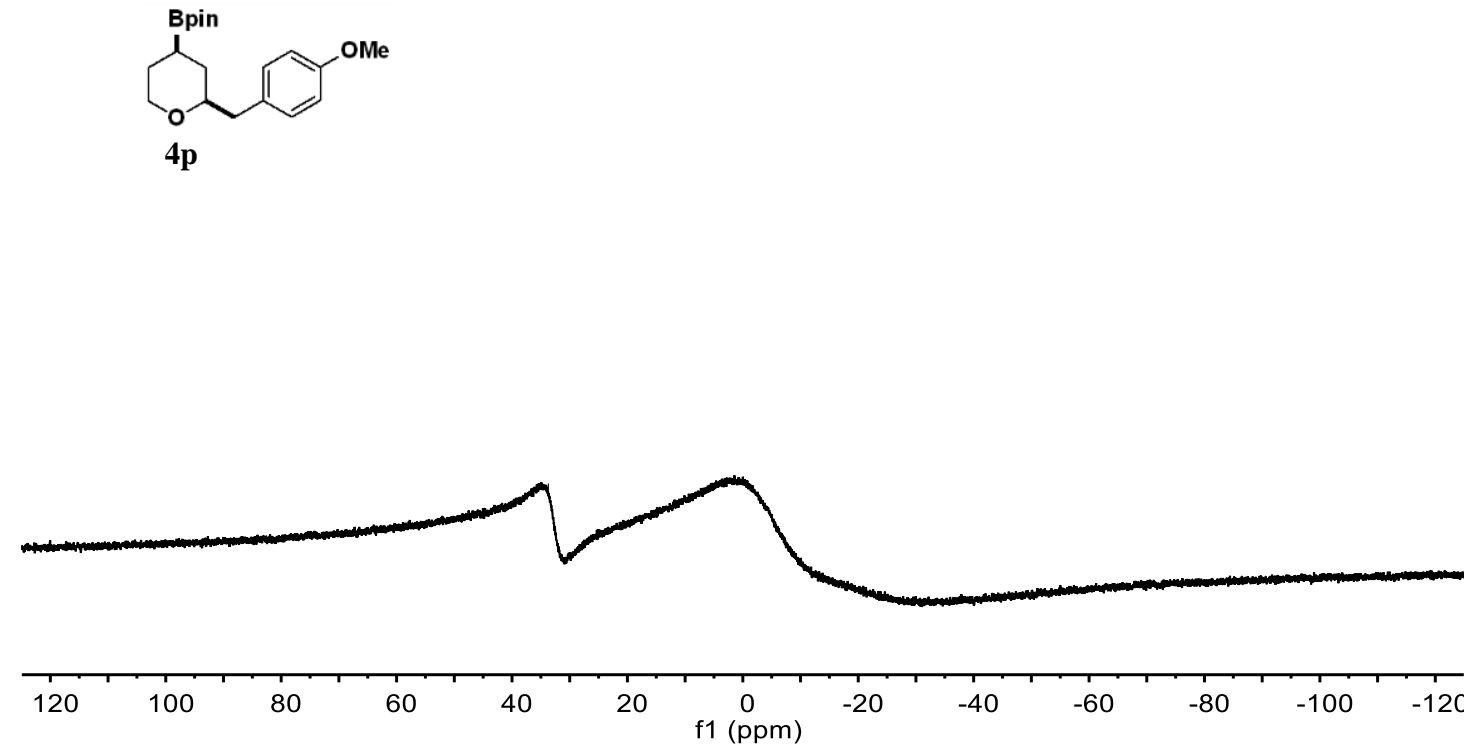

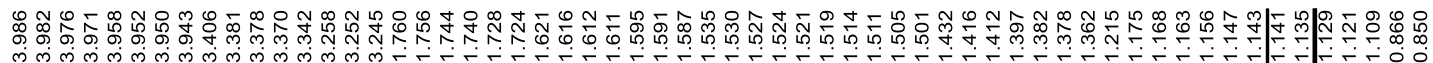

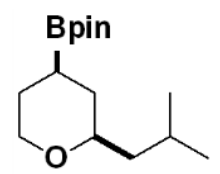

$4 \mathbf{q}$

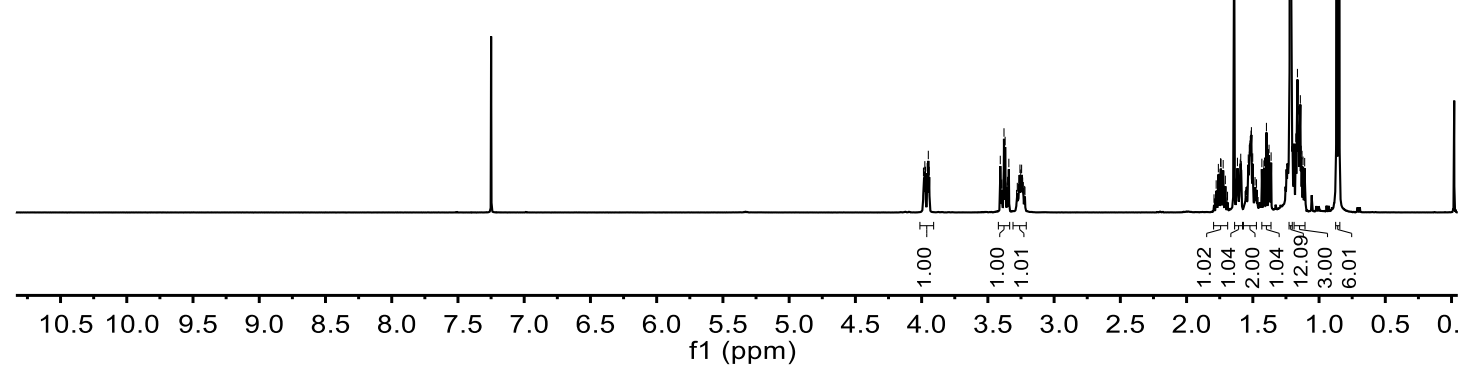



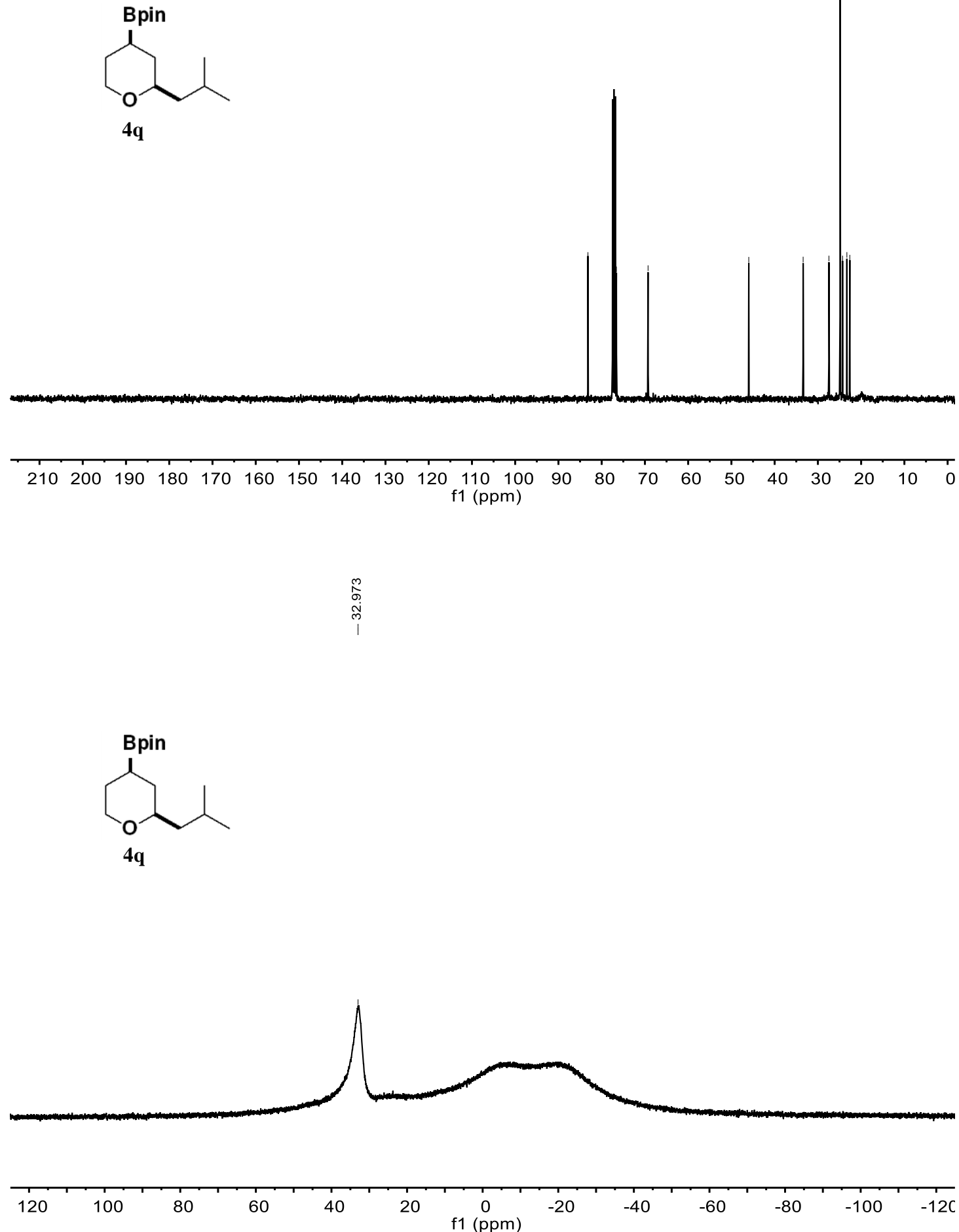
<smiles>c1ccc(CC2CCC(C(c3ccccc3)C3CCOCC3)CC2)cc1</smiles>

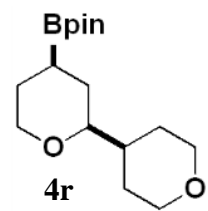

$\begin{array}{lllllllllllllllllllll}210 & 200 & 190 & 180 & 170 & 160 & 150 & 140 & 130 & 120 & 110 & 100 & 90 & 80 & 70 & 60 & 50 & 40 & 30 & 20 & 10\end{array}$ f1 (ppm) 

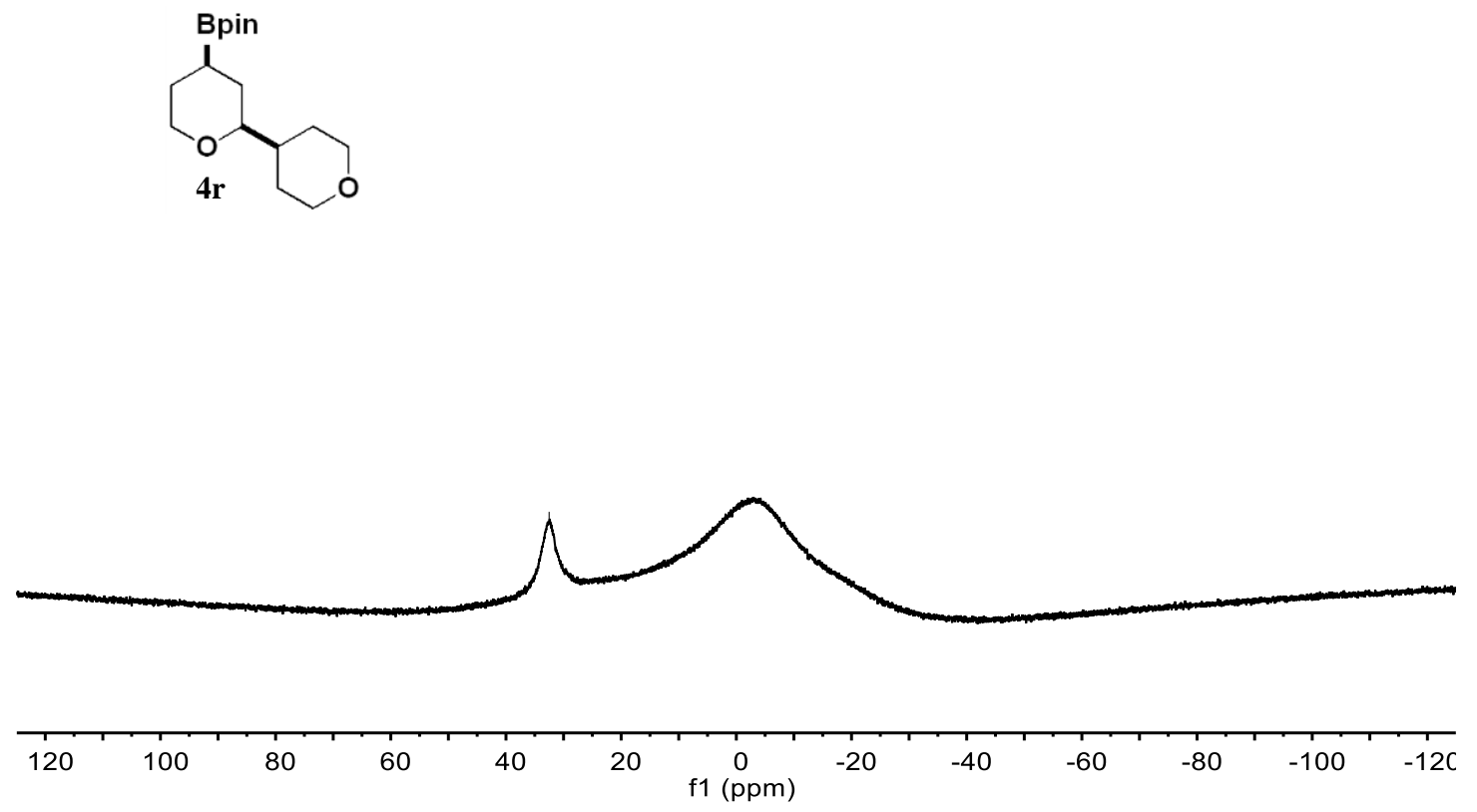

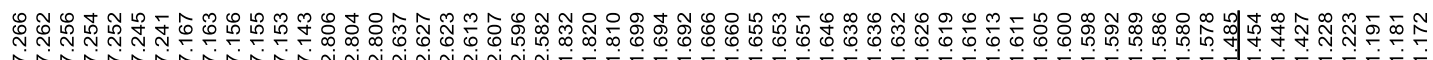

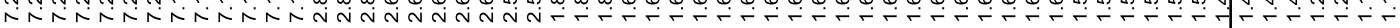<smiles>CC(C)(C)OC(=O)N1CCC(Br)CC1CCCc1ccccc1</smiles>

$4 s$

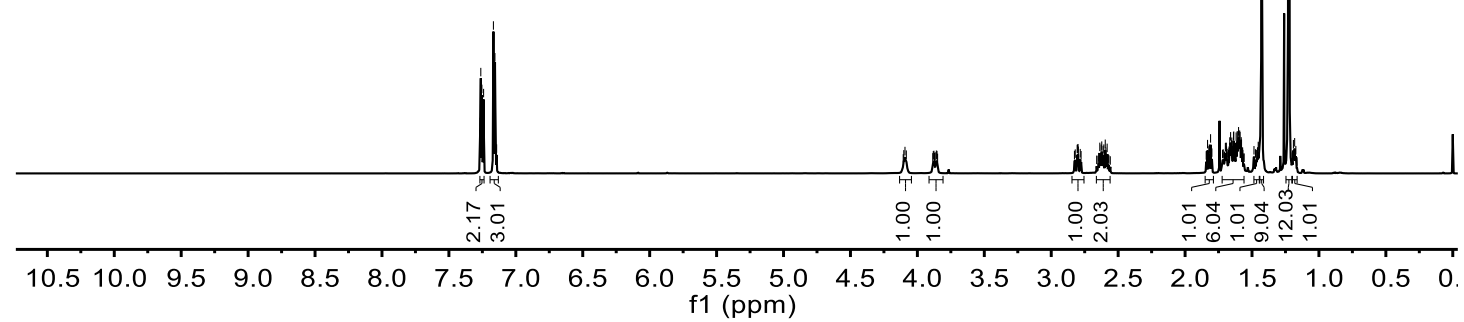



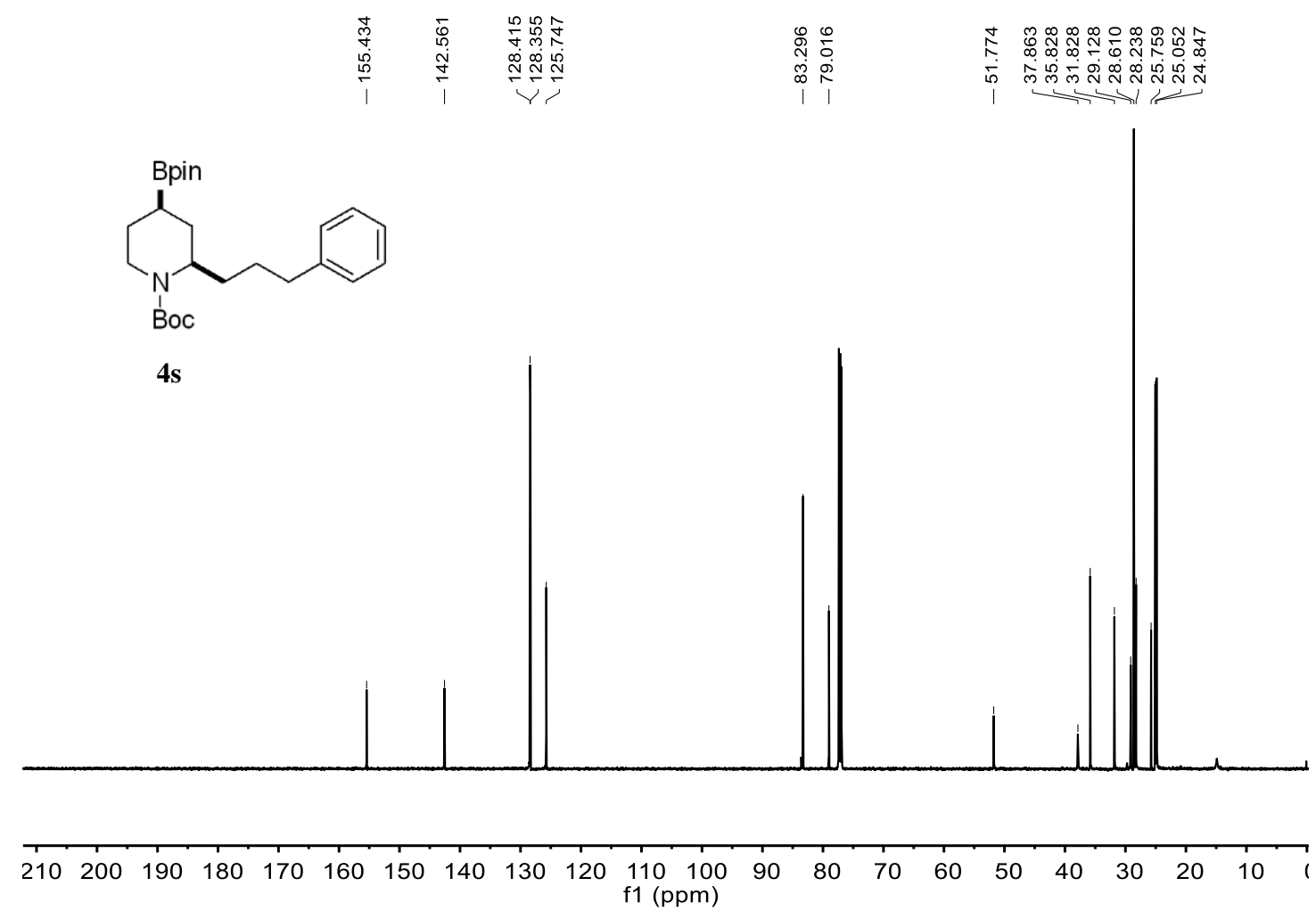<smiles>O=C(c1ccccc1)N1CCC(Cc2ccccc2)CC1CCCc1ccccc1</smiles>

$4 s$

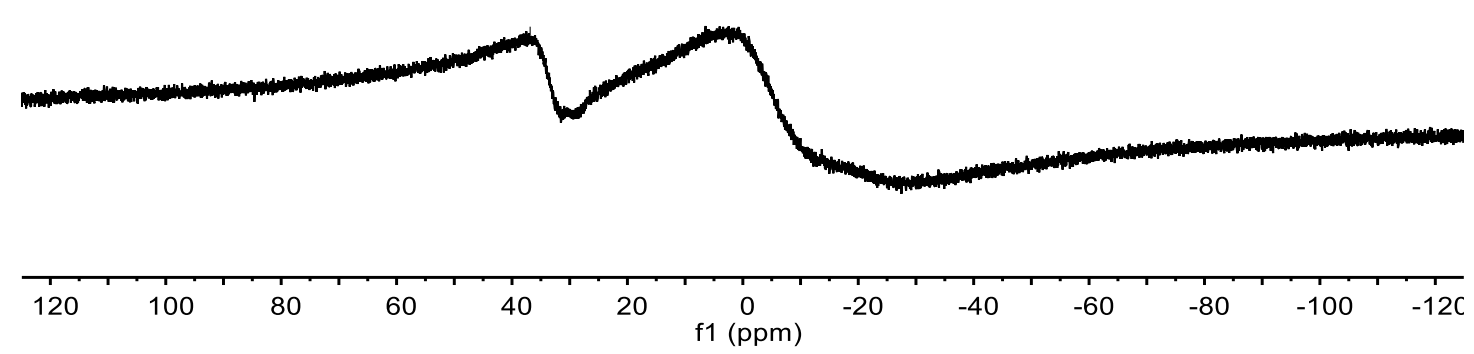



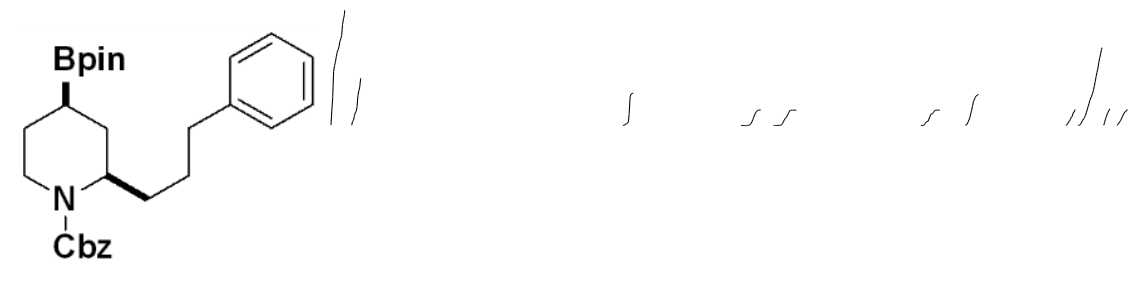

$4 \mathrm{t}$

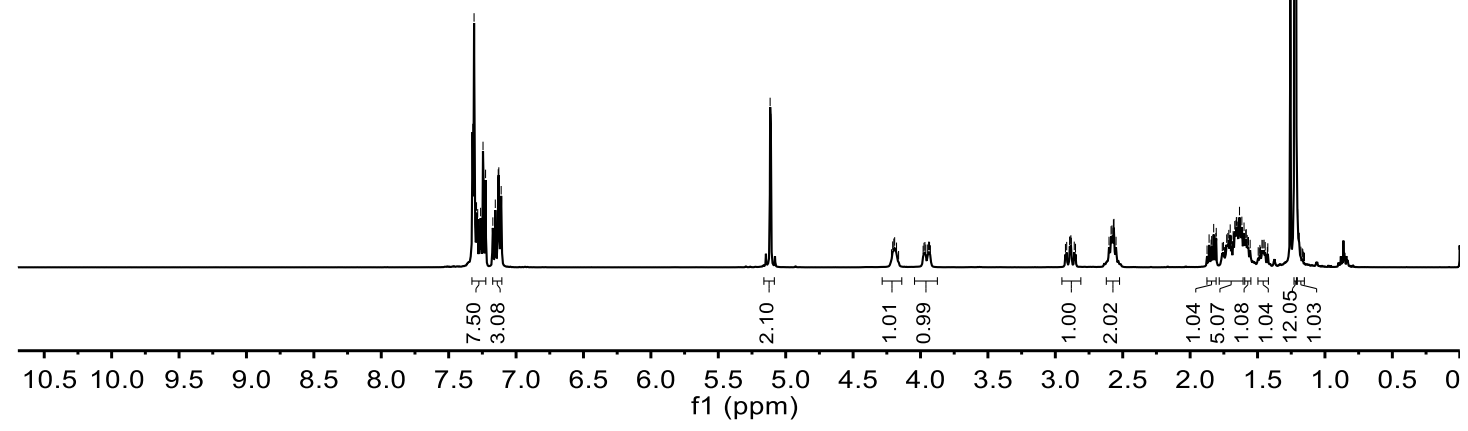

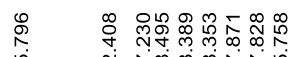

至

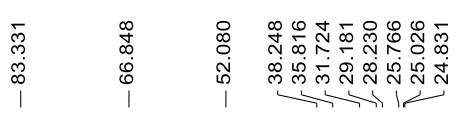

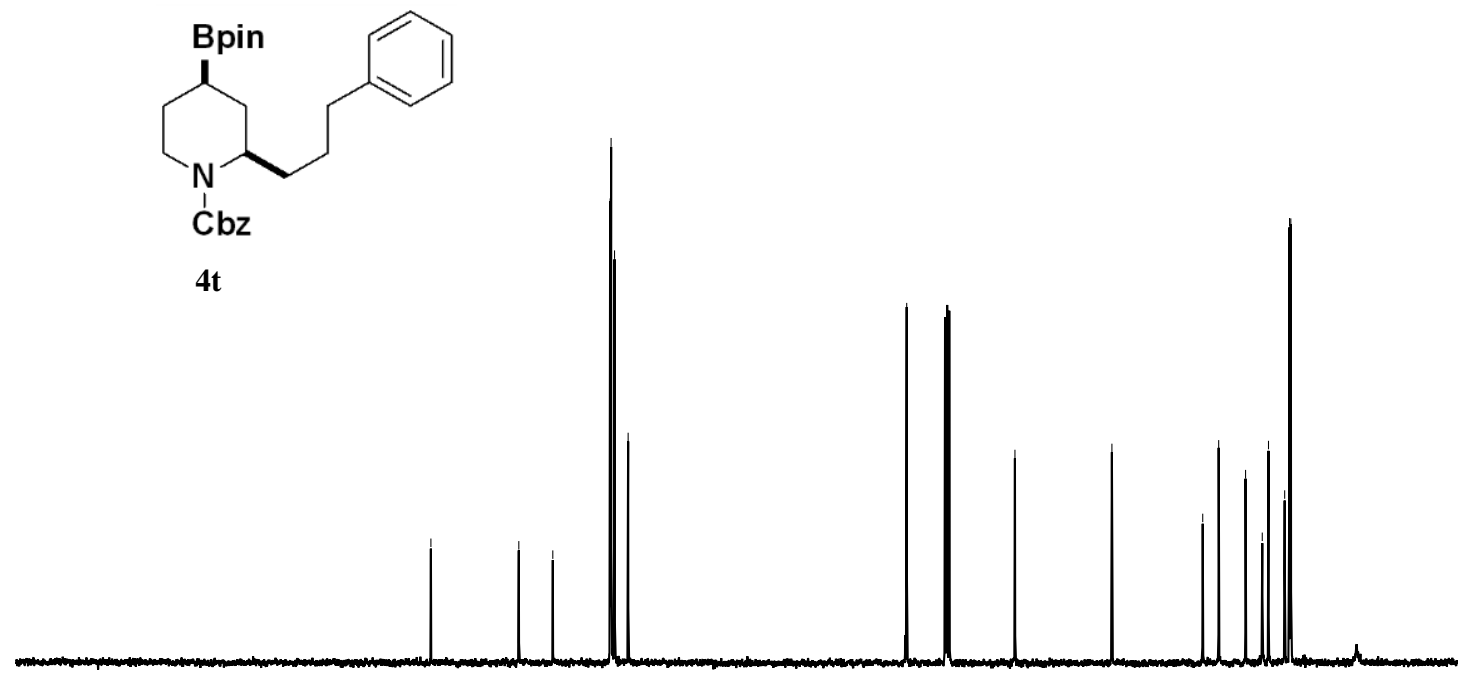

$\begin{array}{lllllllllllllllllllll}210 & 200 & 190 & 180 & 170 & 160 & 150 & 140 & 130 & 120 & 110 & 100 & 90 & 80 & 70 & 60 & 50 & 40 & 30 & 20 & 10\end{array}$ f1 (ppm) 


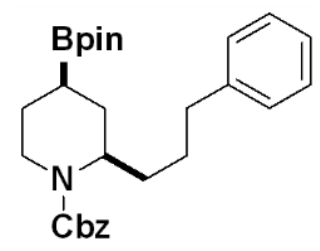

4t
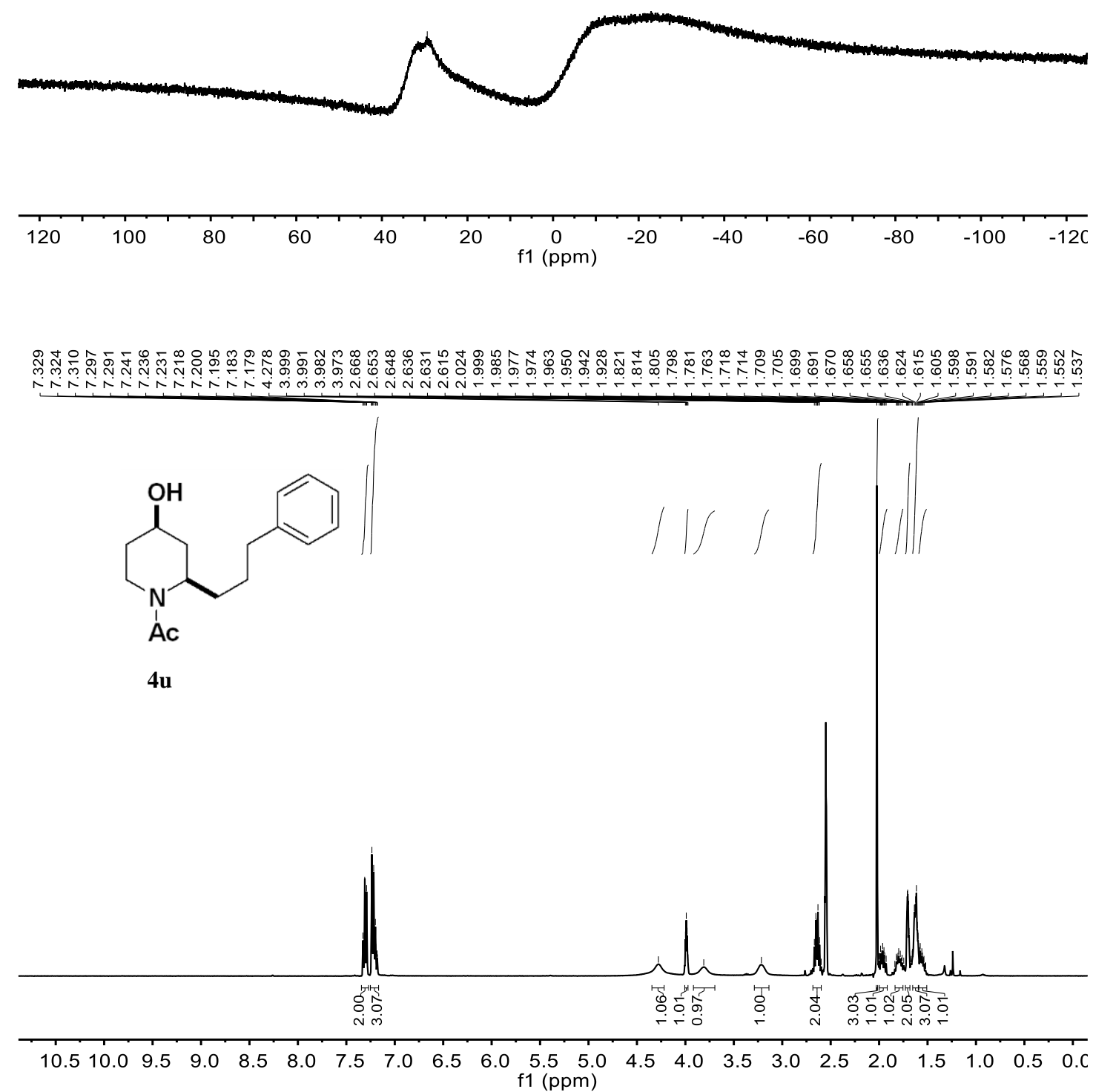


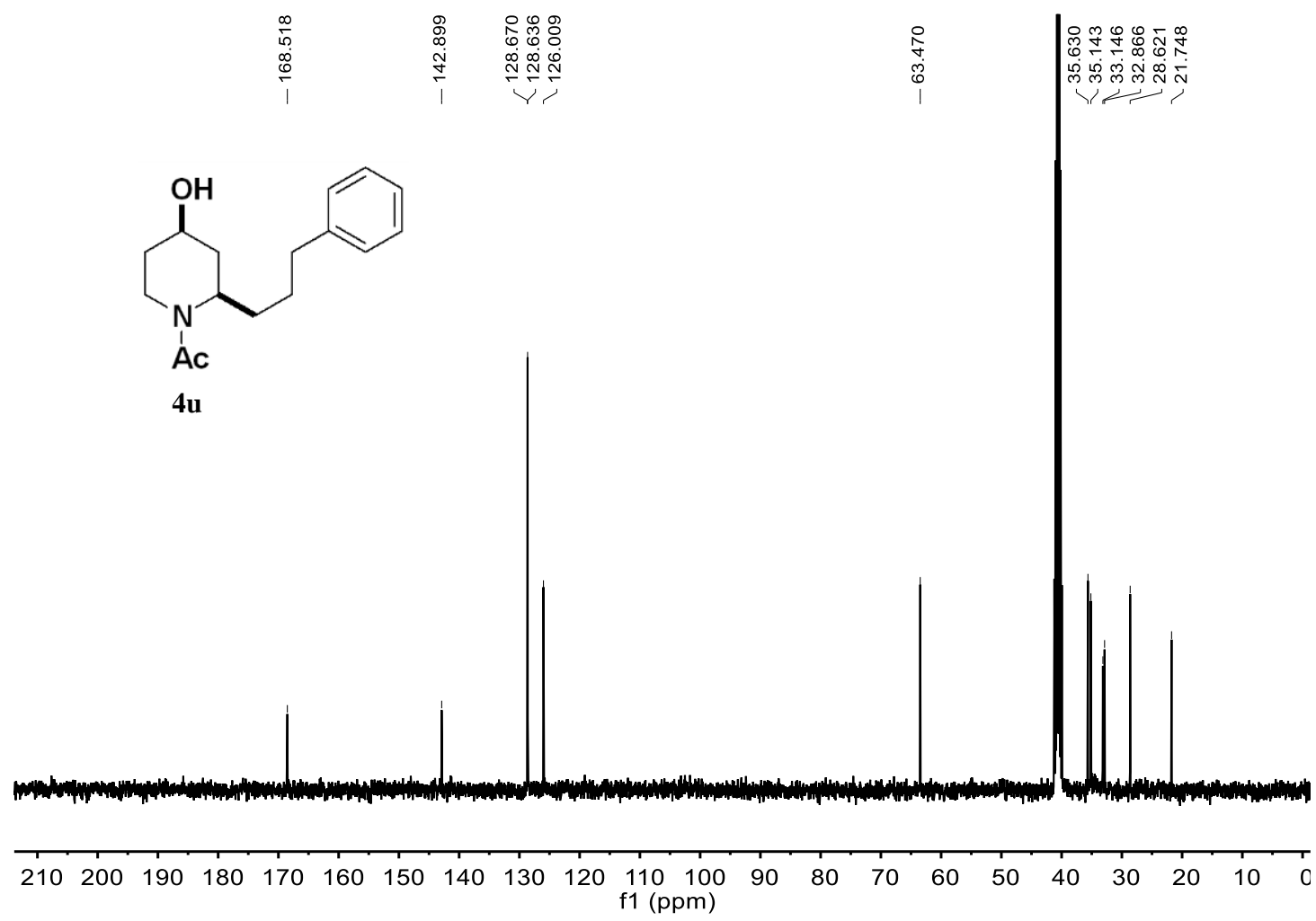

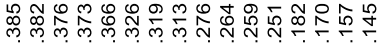

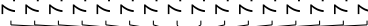

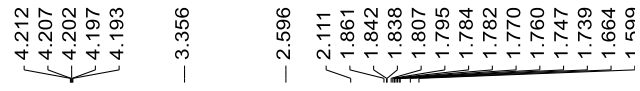

$\overbrace{\substack{1 \\ \mathrm{Bz}}}^{\mathrm{OH}}$

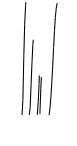

$4 v$

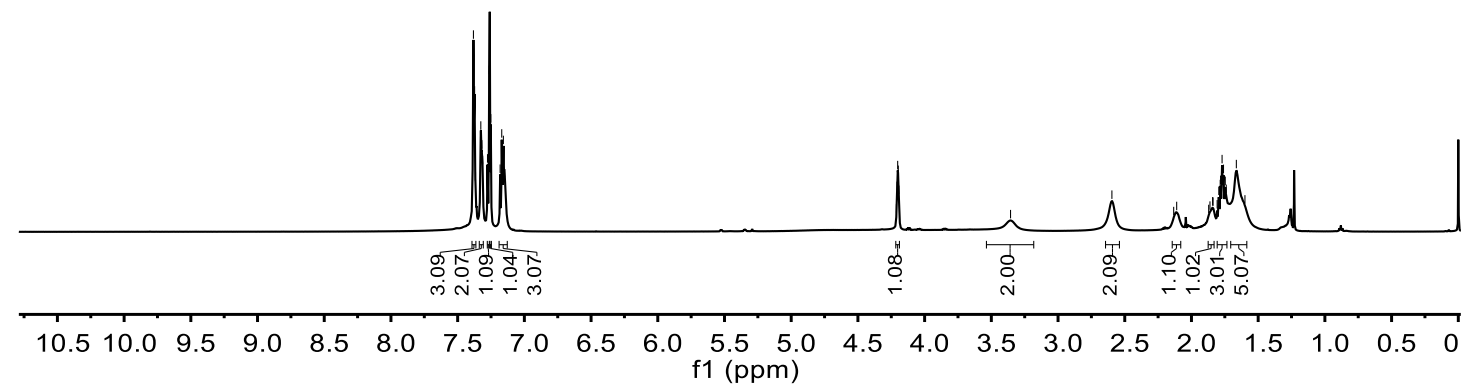




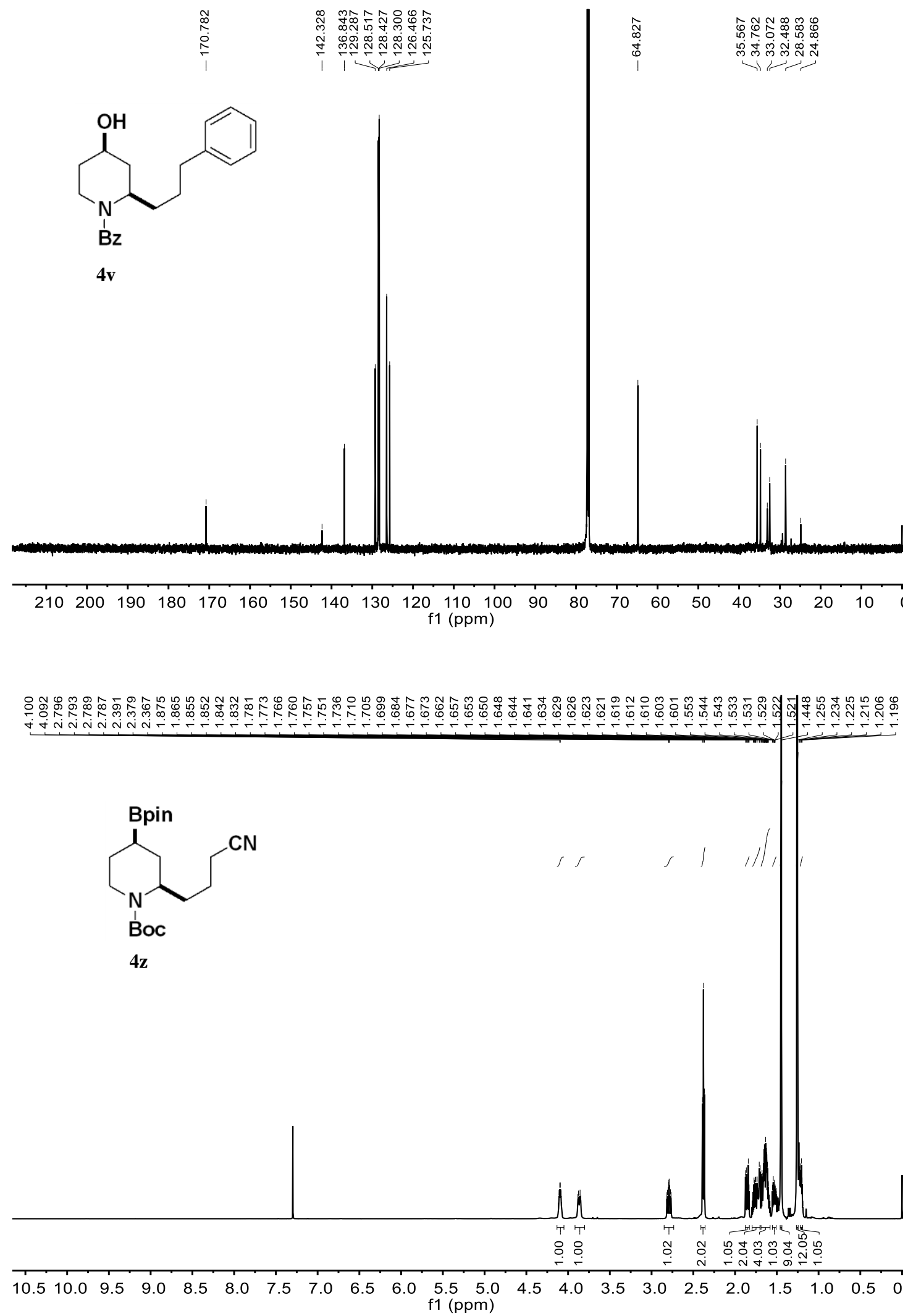



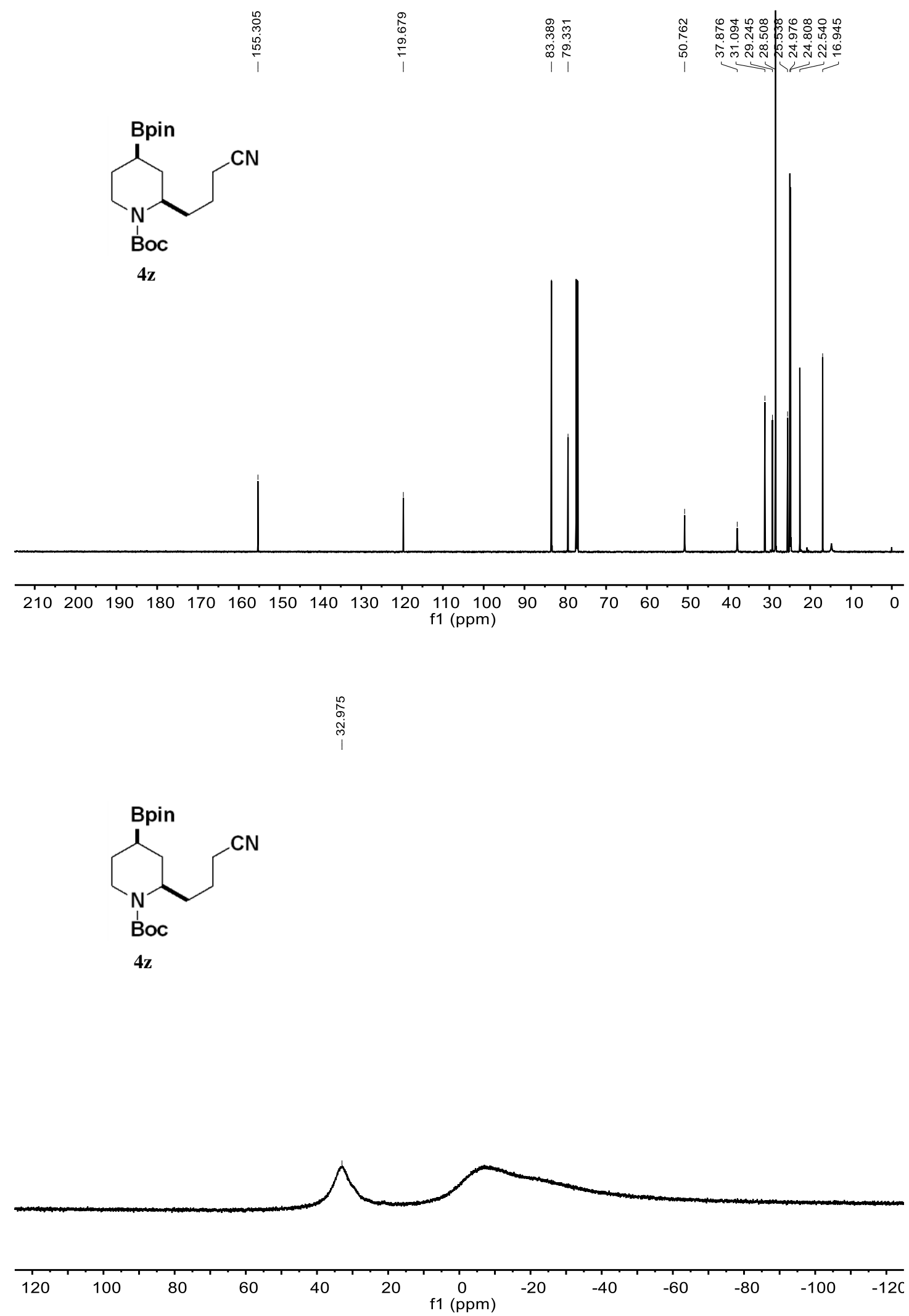

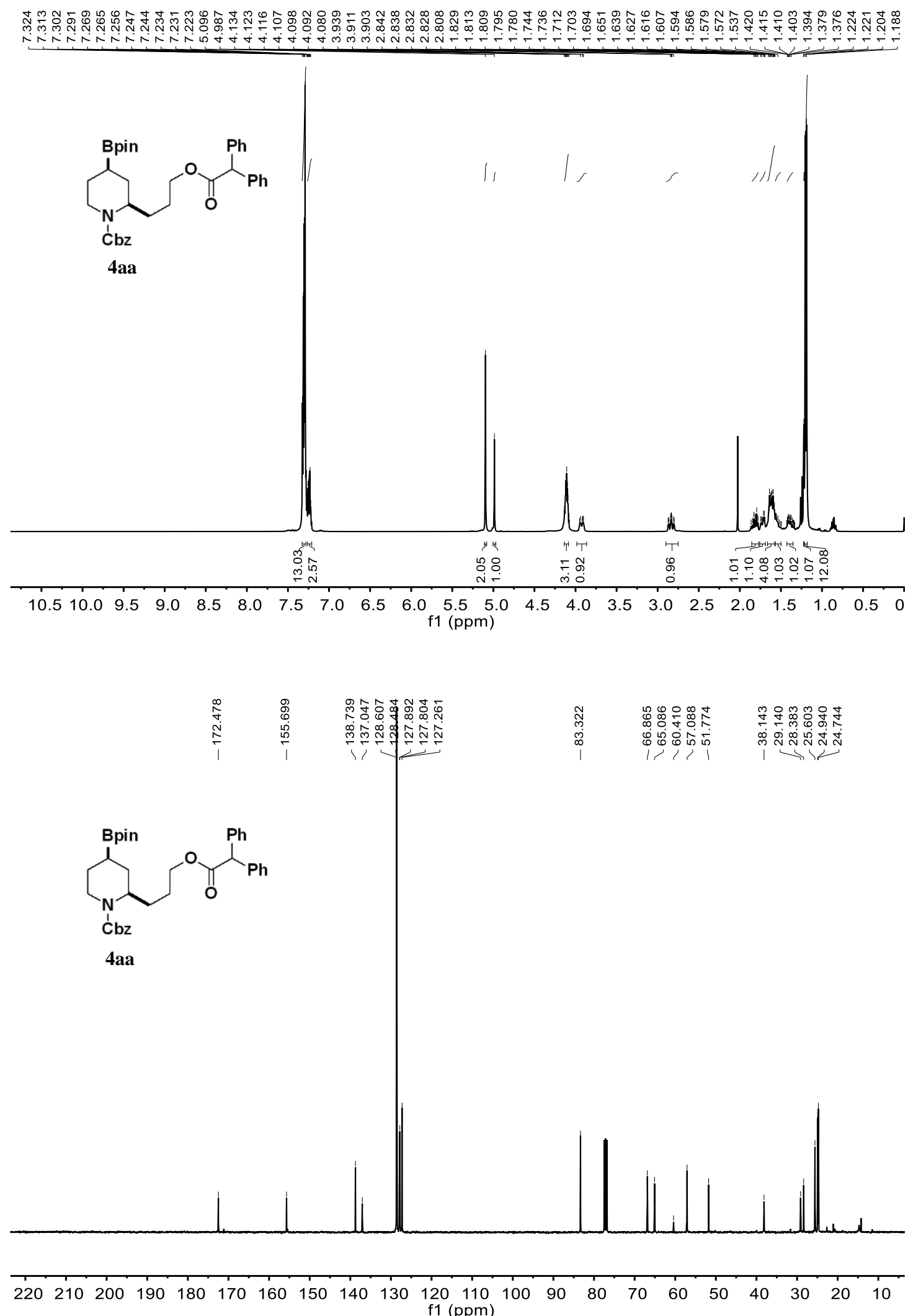


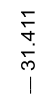<smiles>[14CH3]N1CCC([B]c2ccccc2)CC1CCCC(=O)C(c1ccccc1)c1ccccc1</smiles>

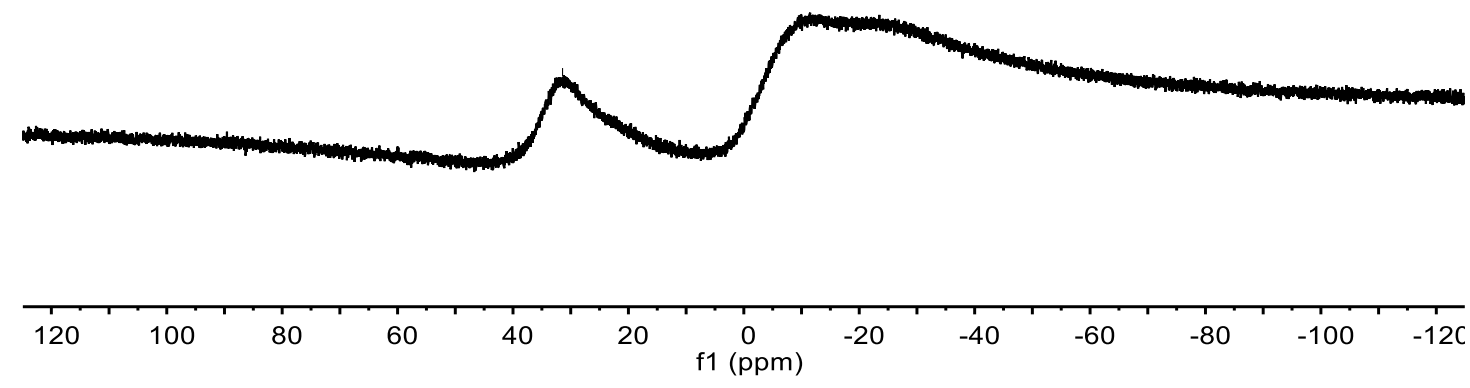

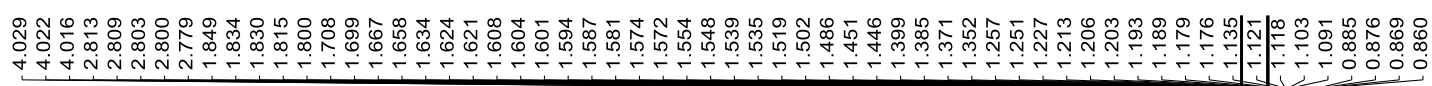

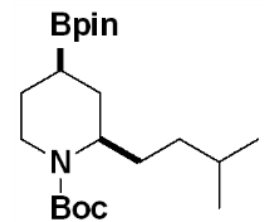

4ab

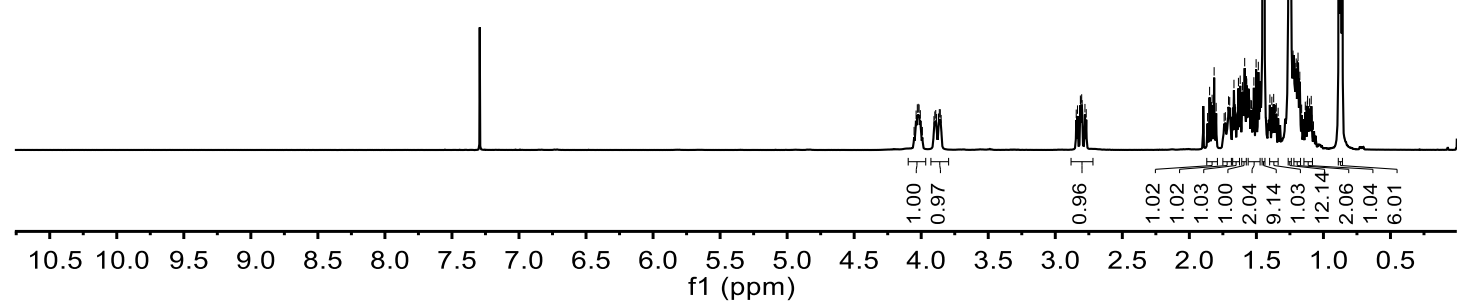



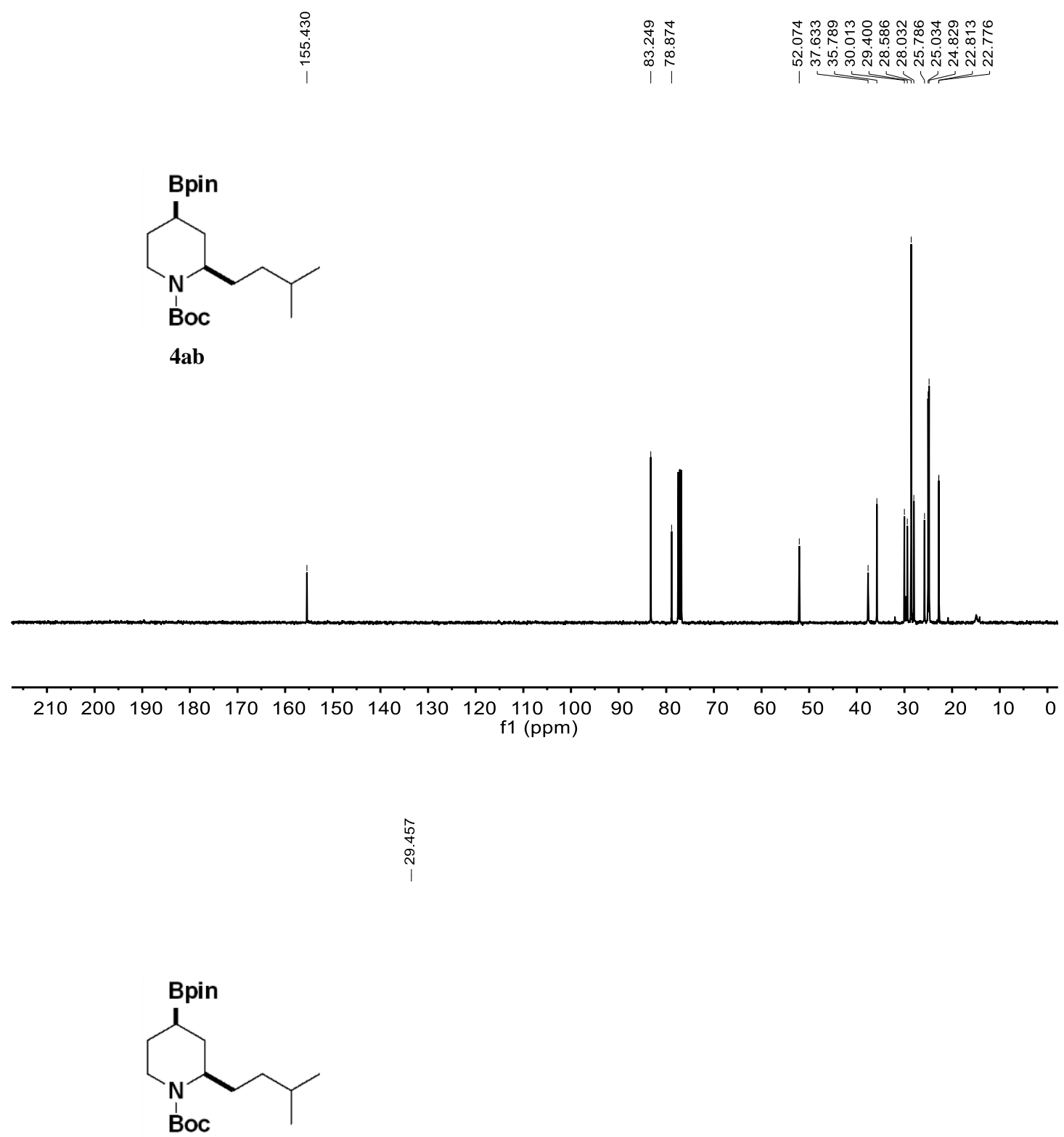

4ab
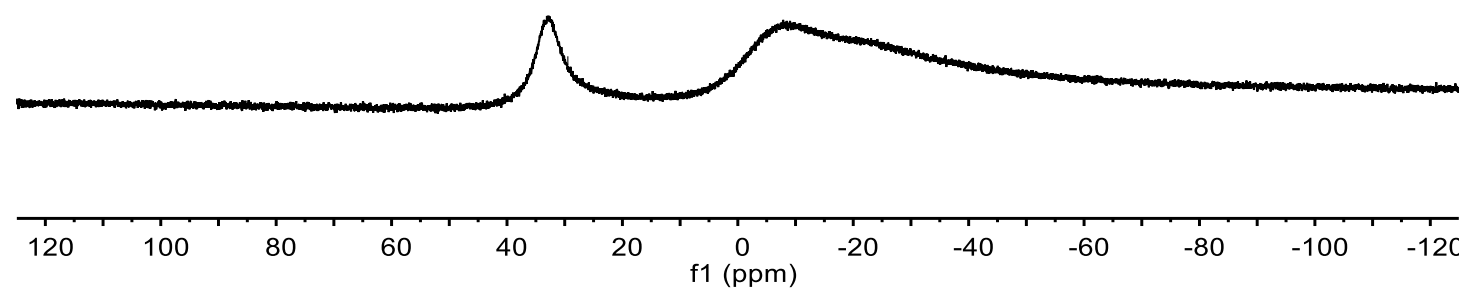
<smiles>O=C(c1ccccc1)N1CCC([B]c2ccccc2)CC1CCc1ccc2ccccc2c1</smiles>

$4 a c$
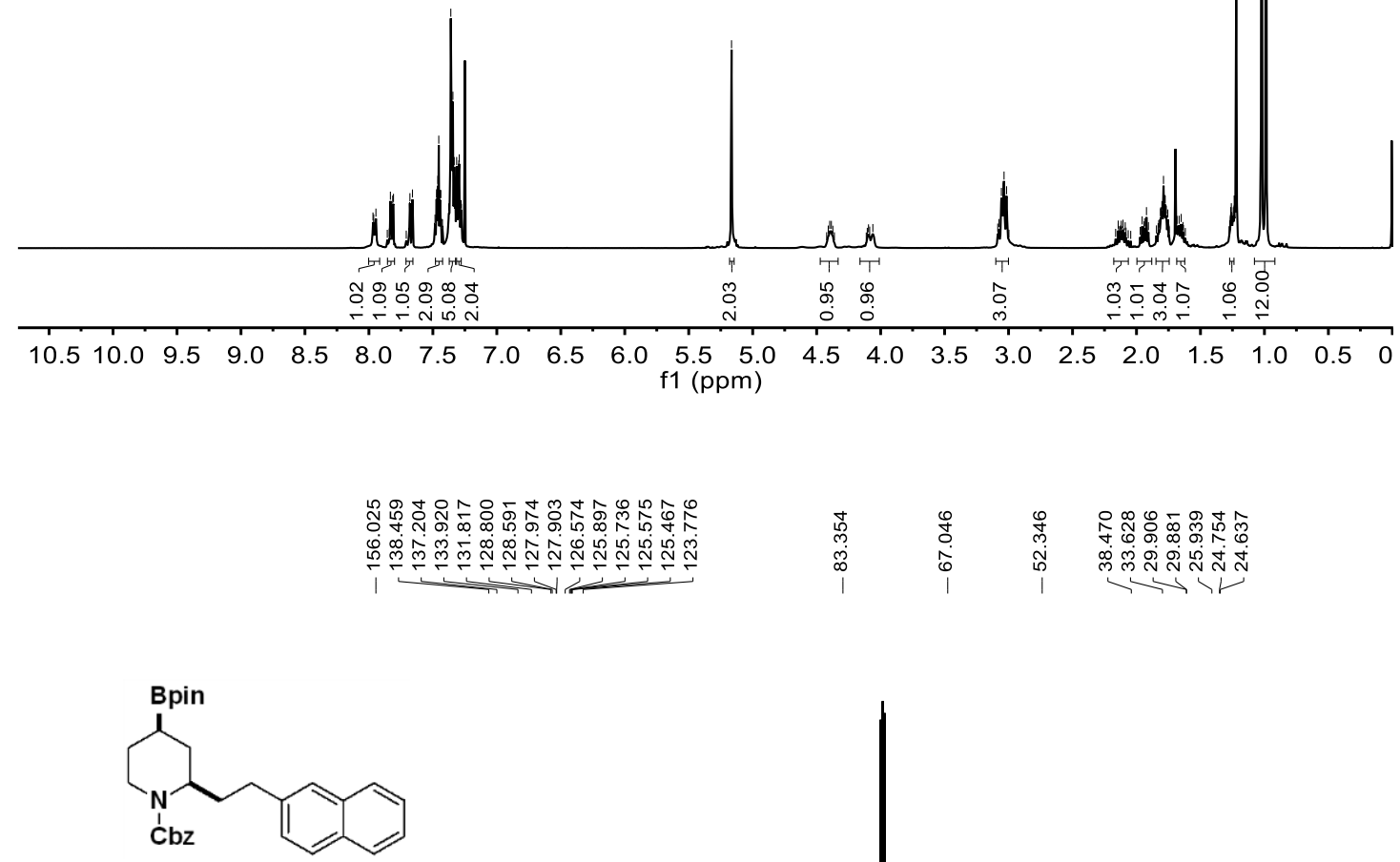

$4 a c$

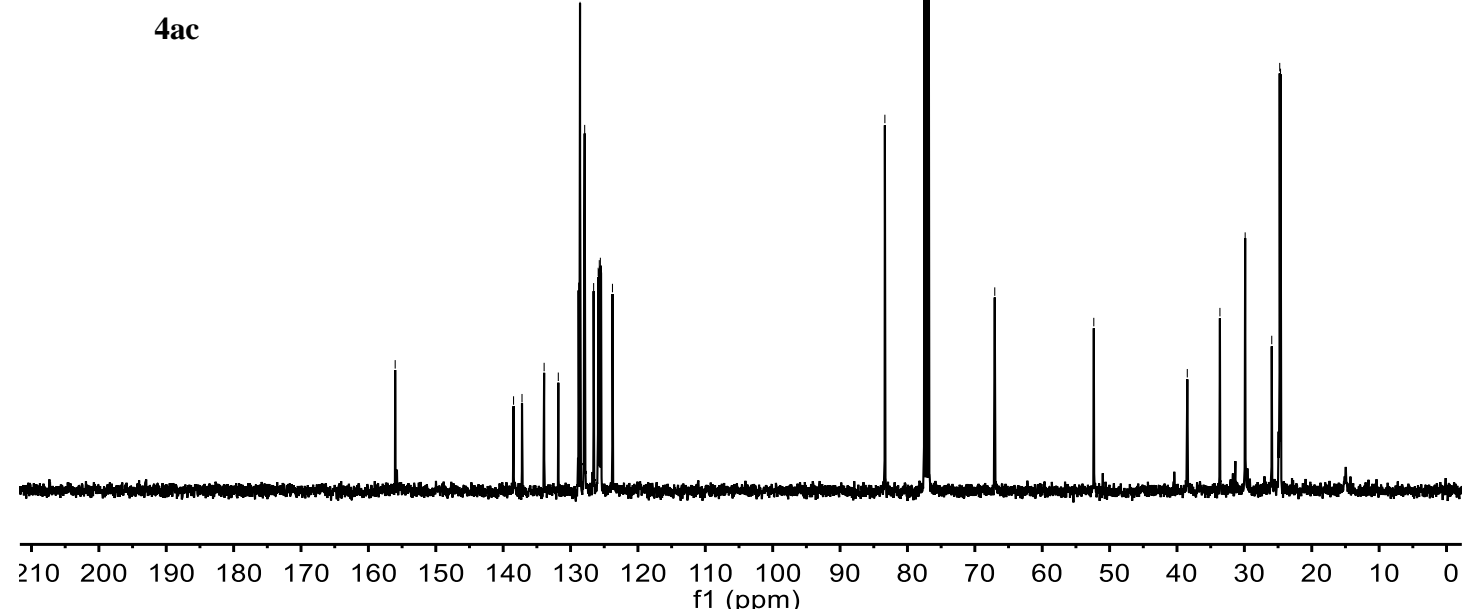




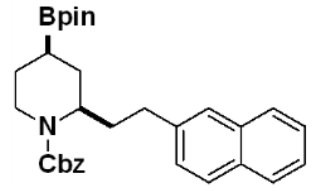

$4 a c$
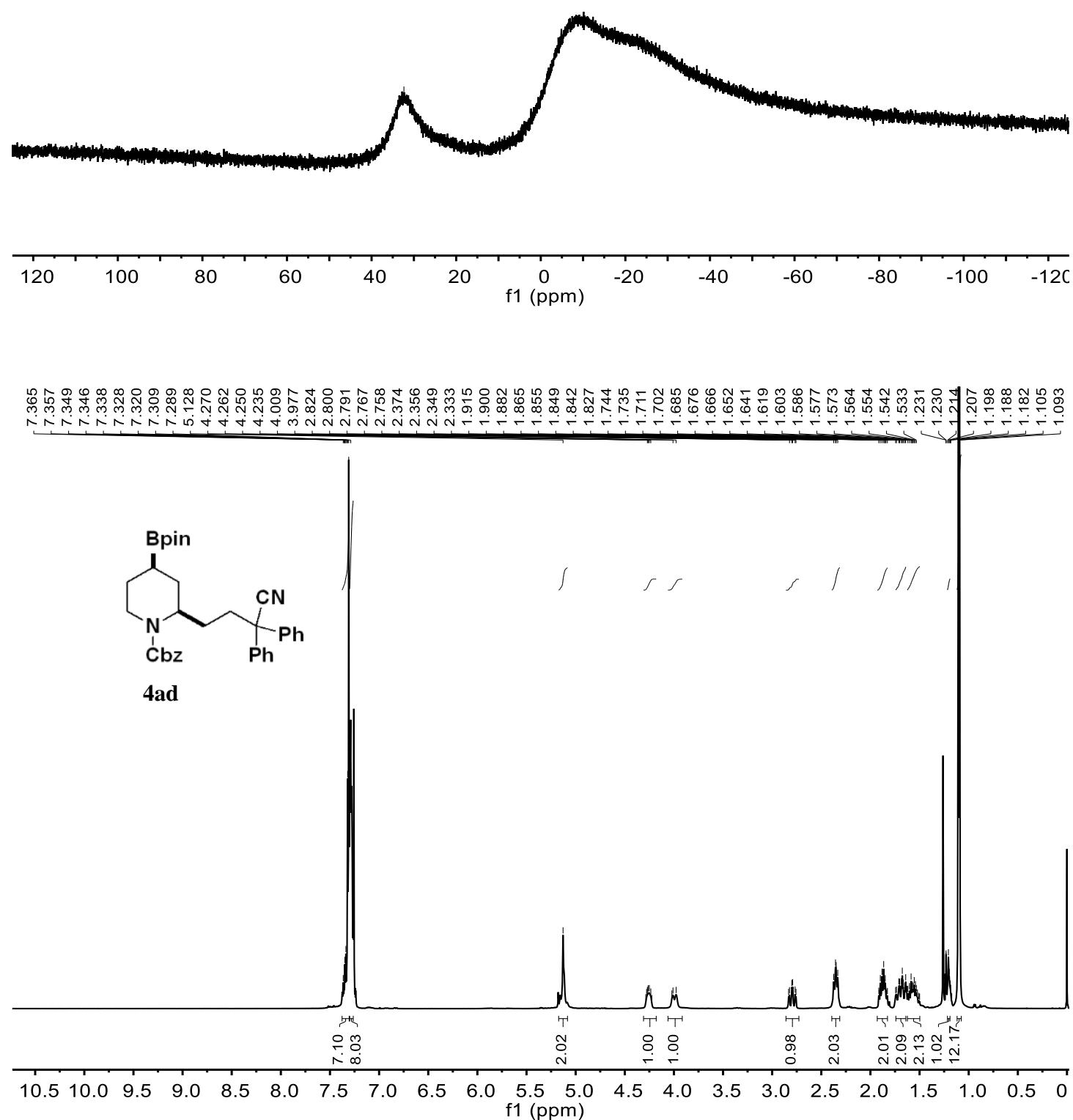

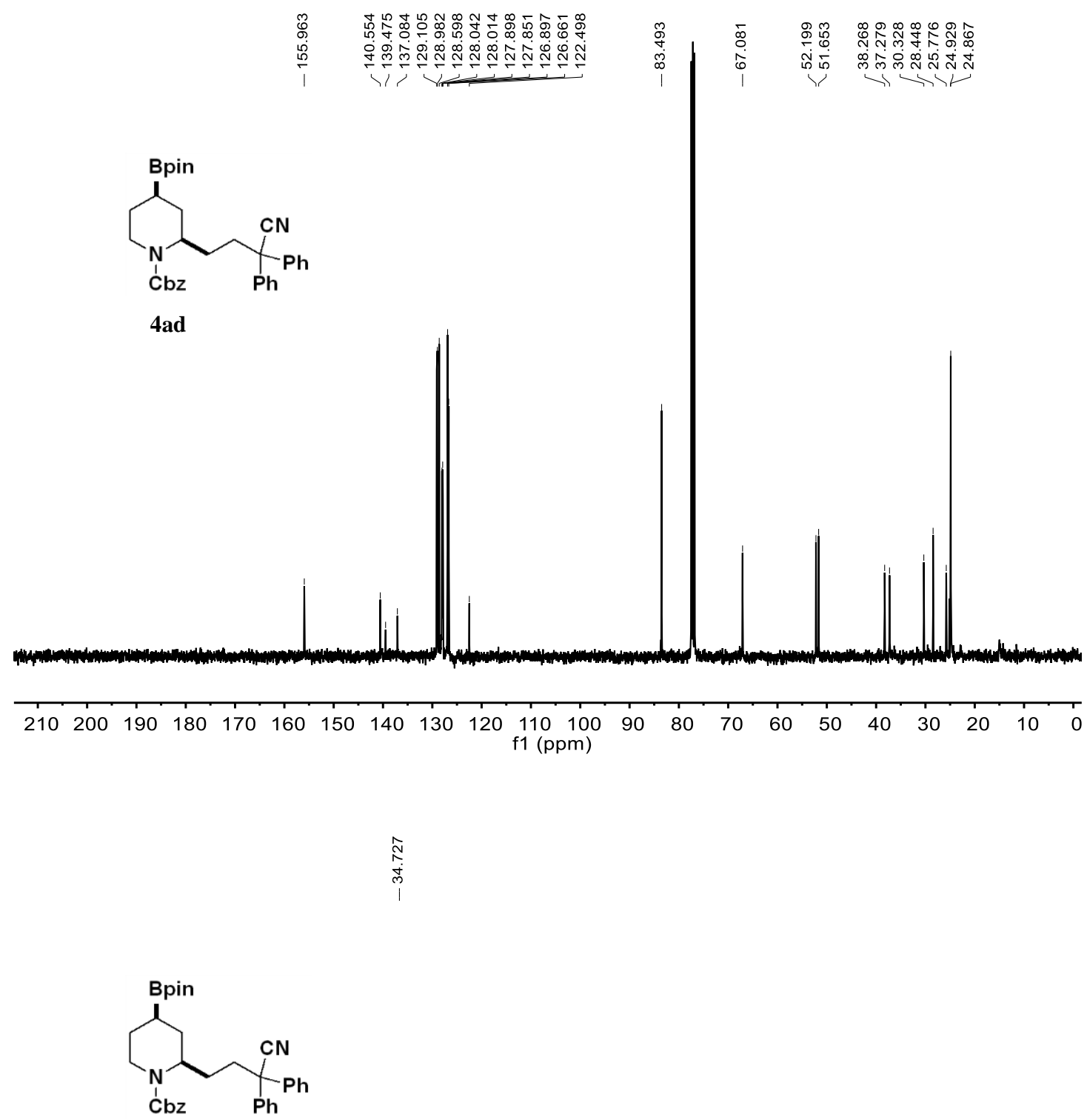

4ad

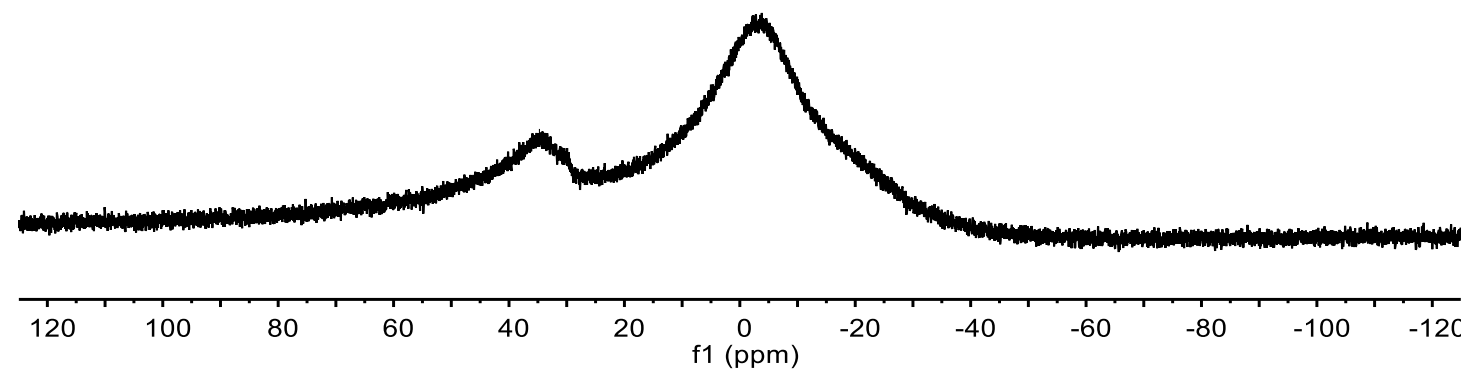



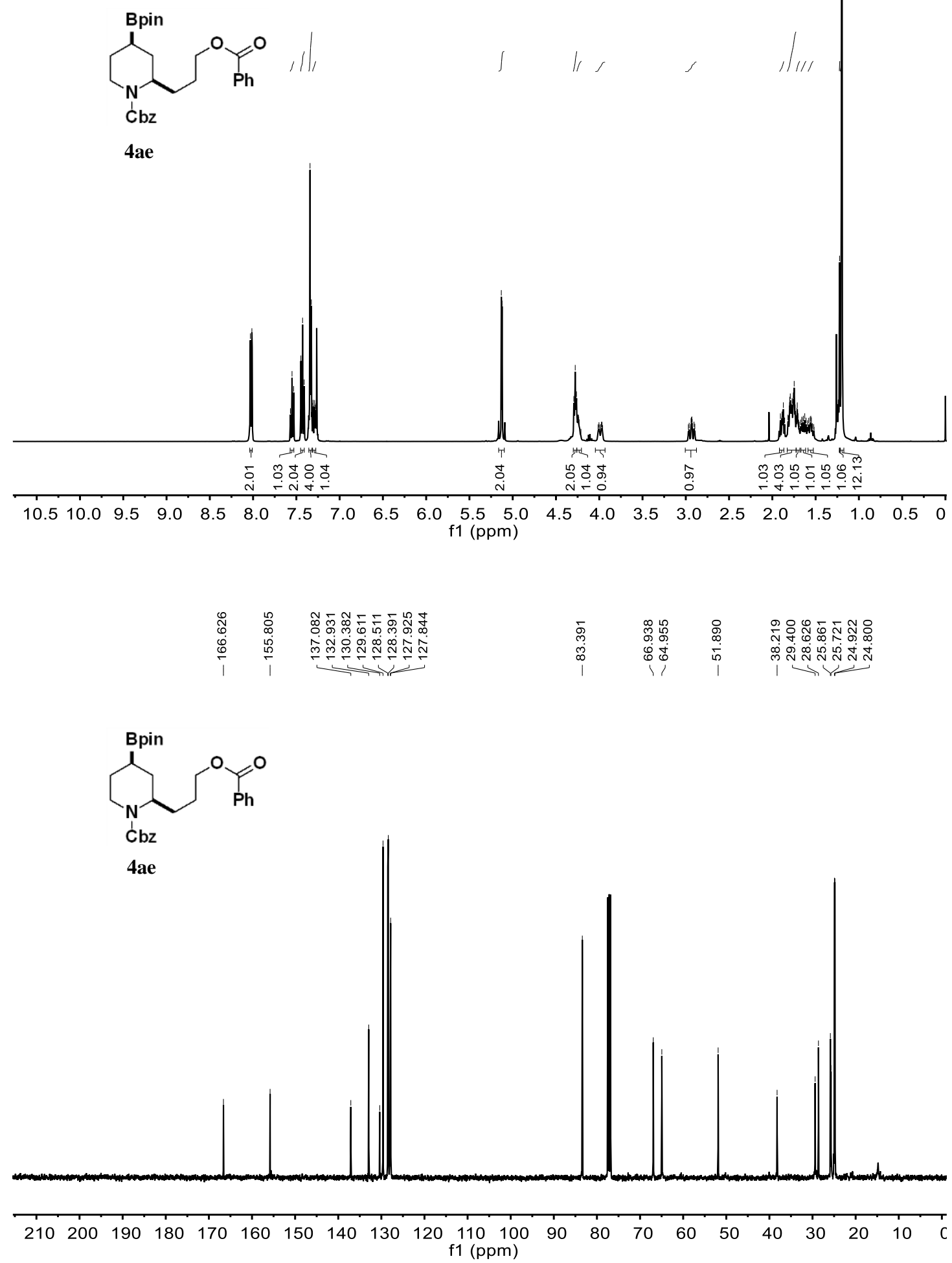


\section{!}
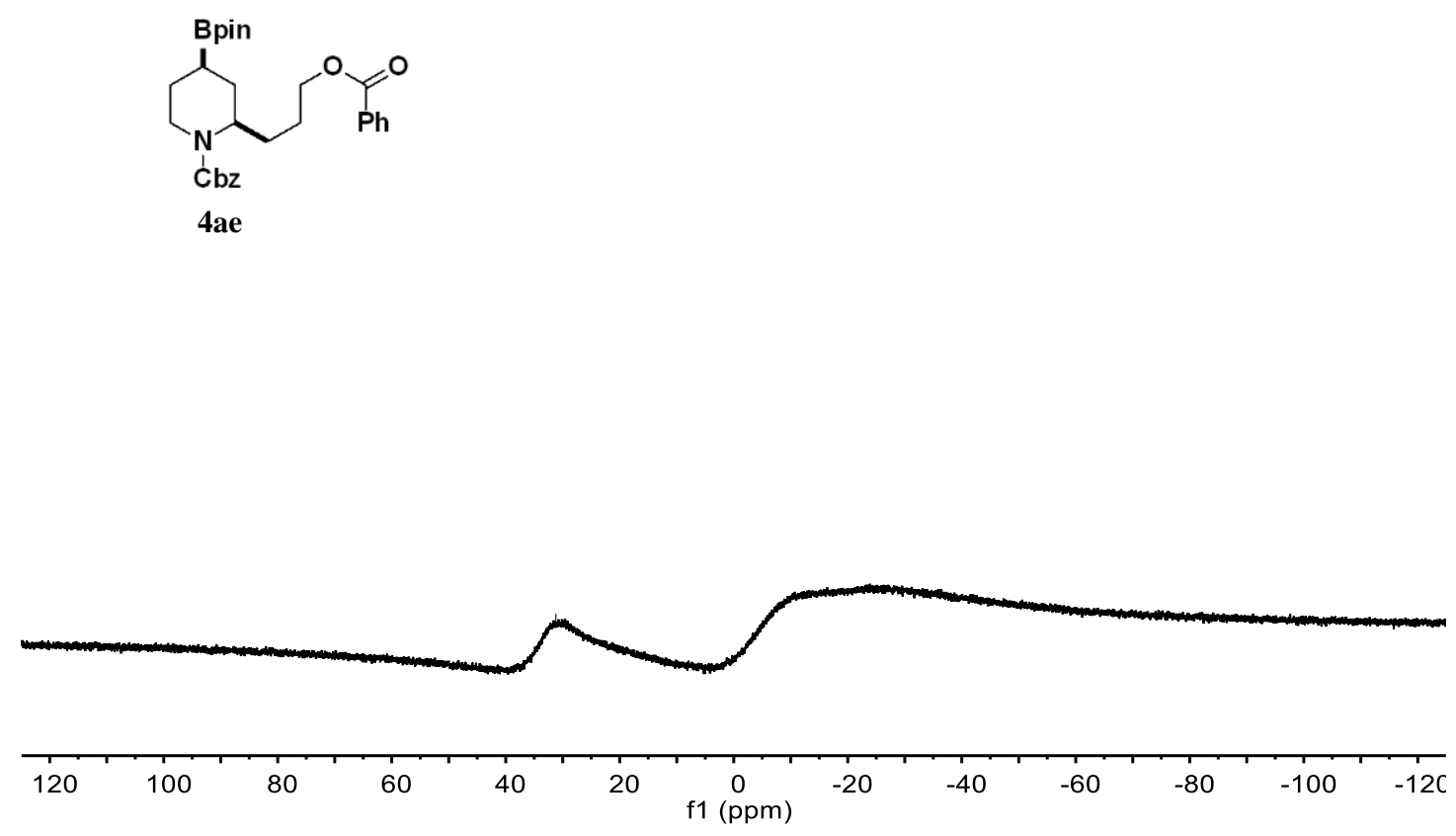

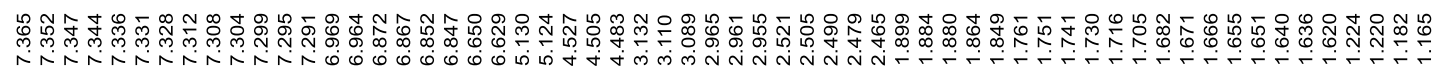

risis
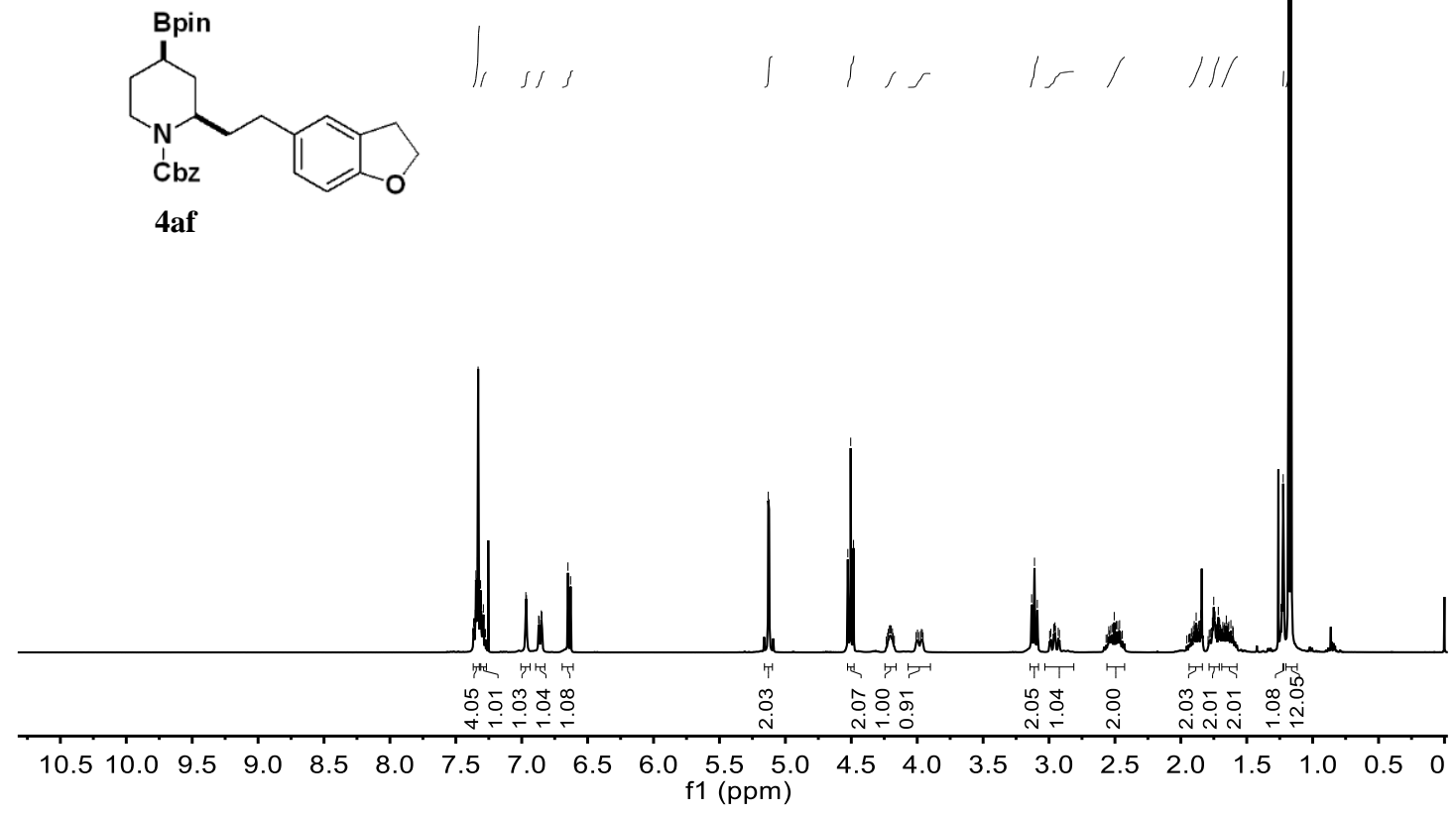

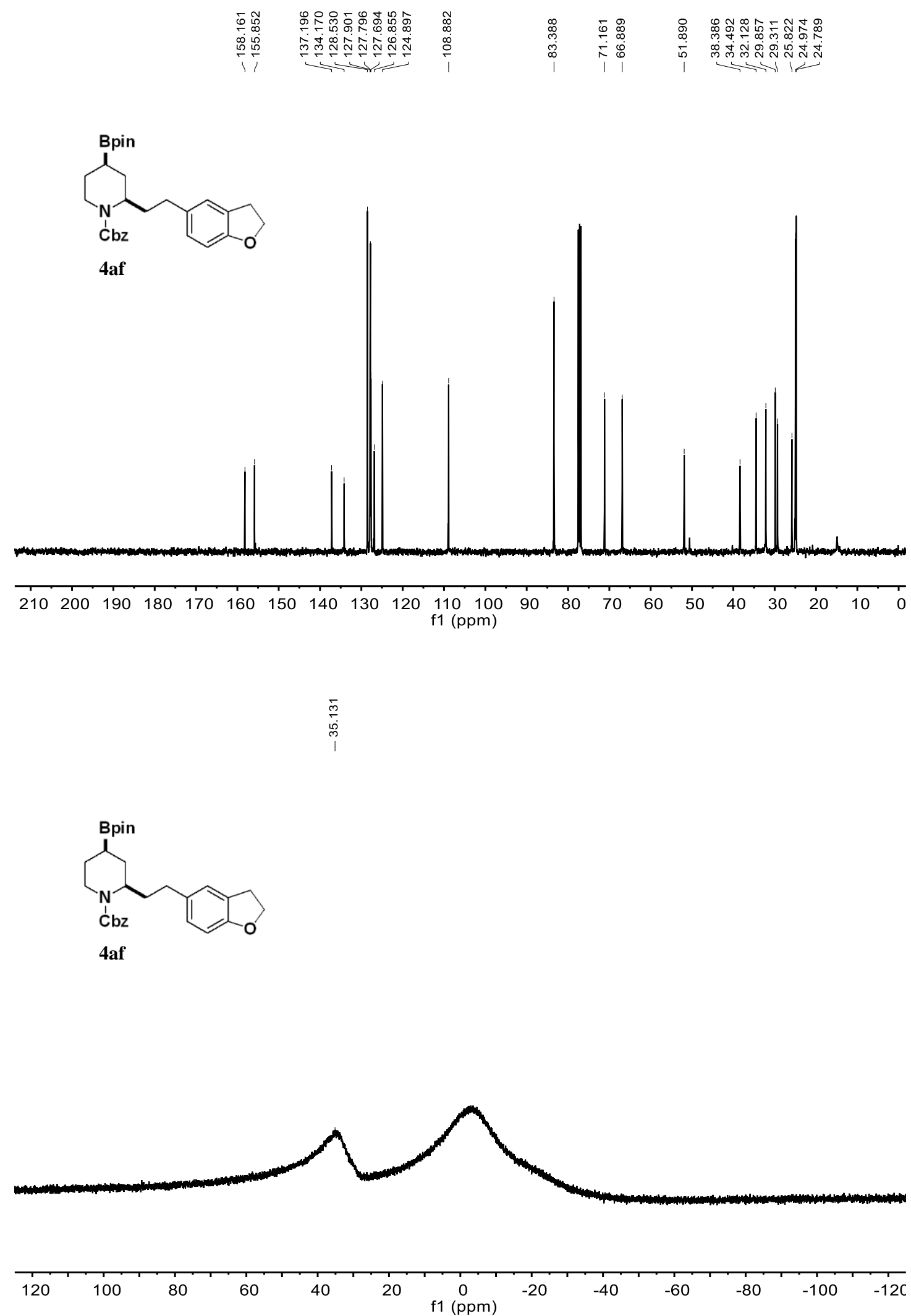

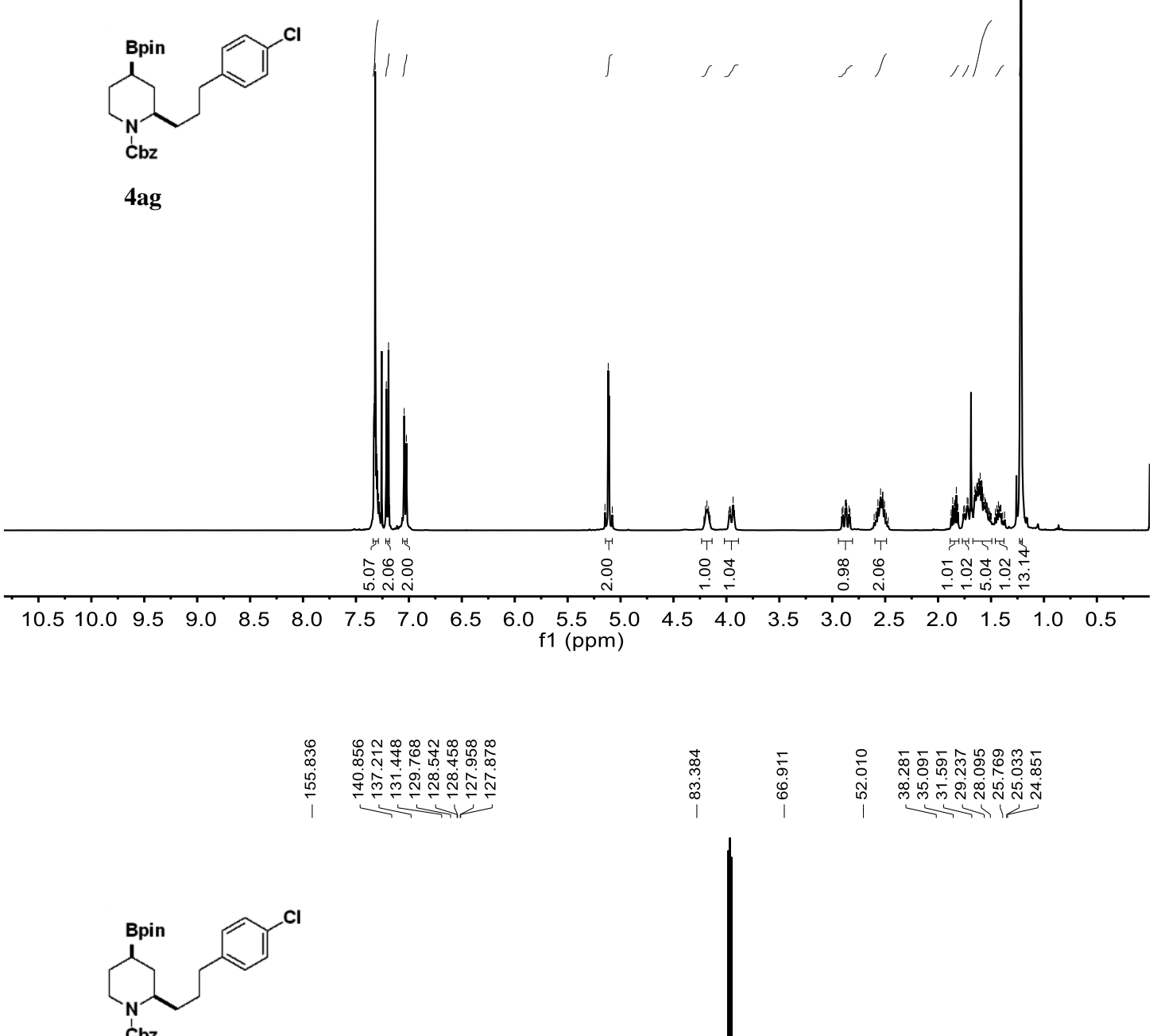

4ag

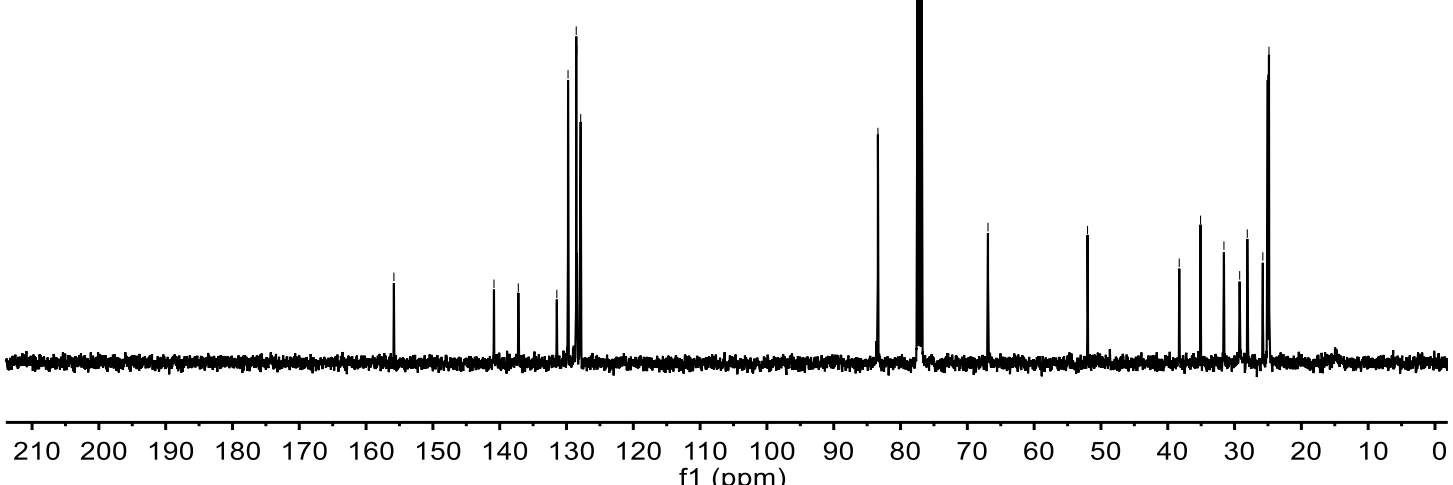




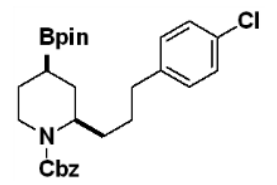

$4 a g$
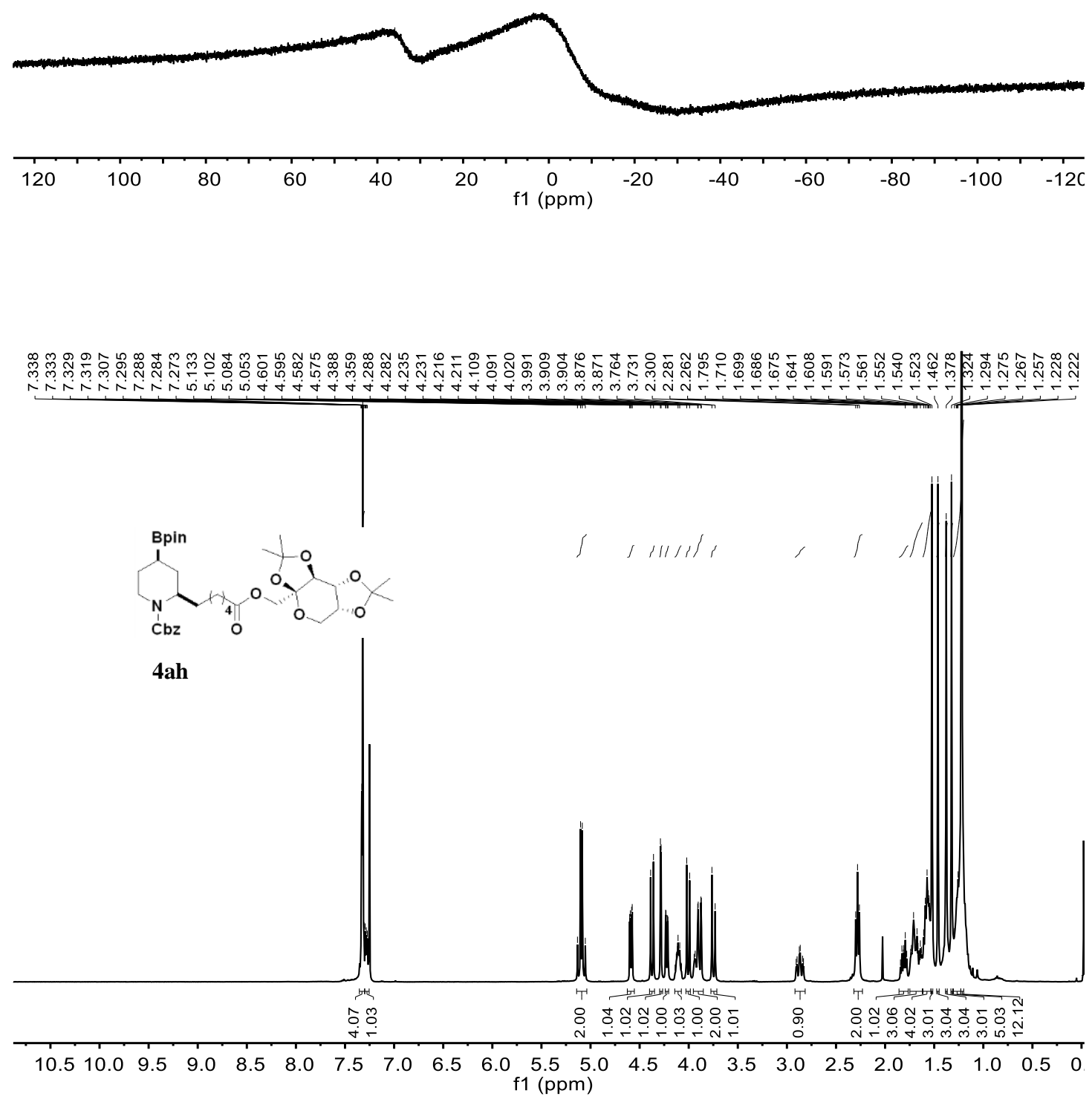


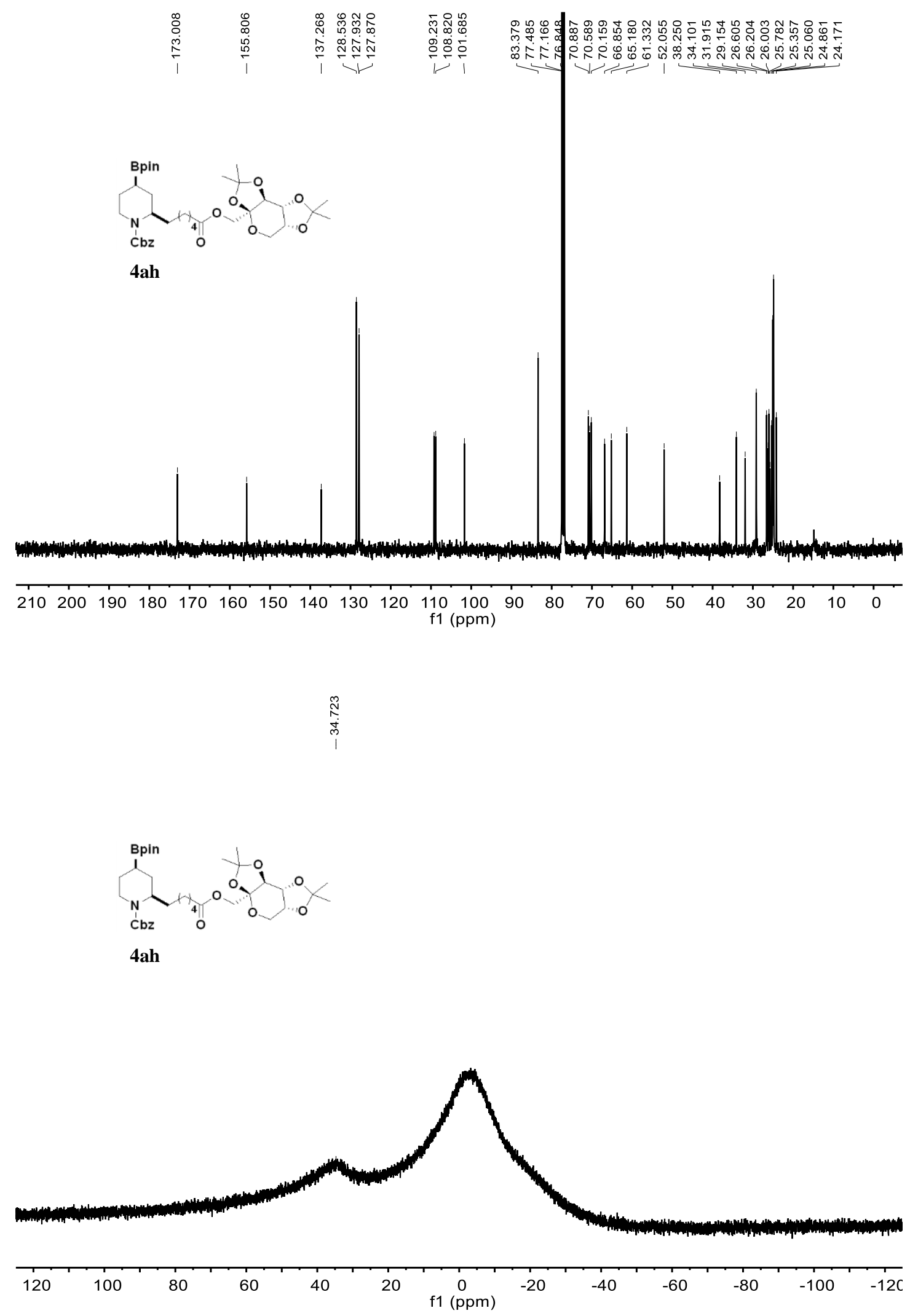



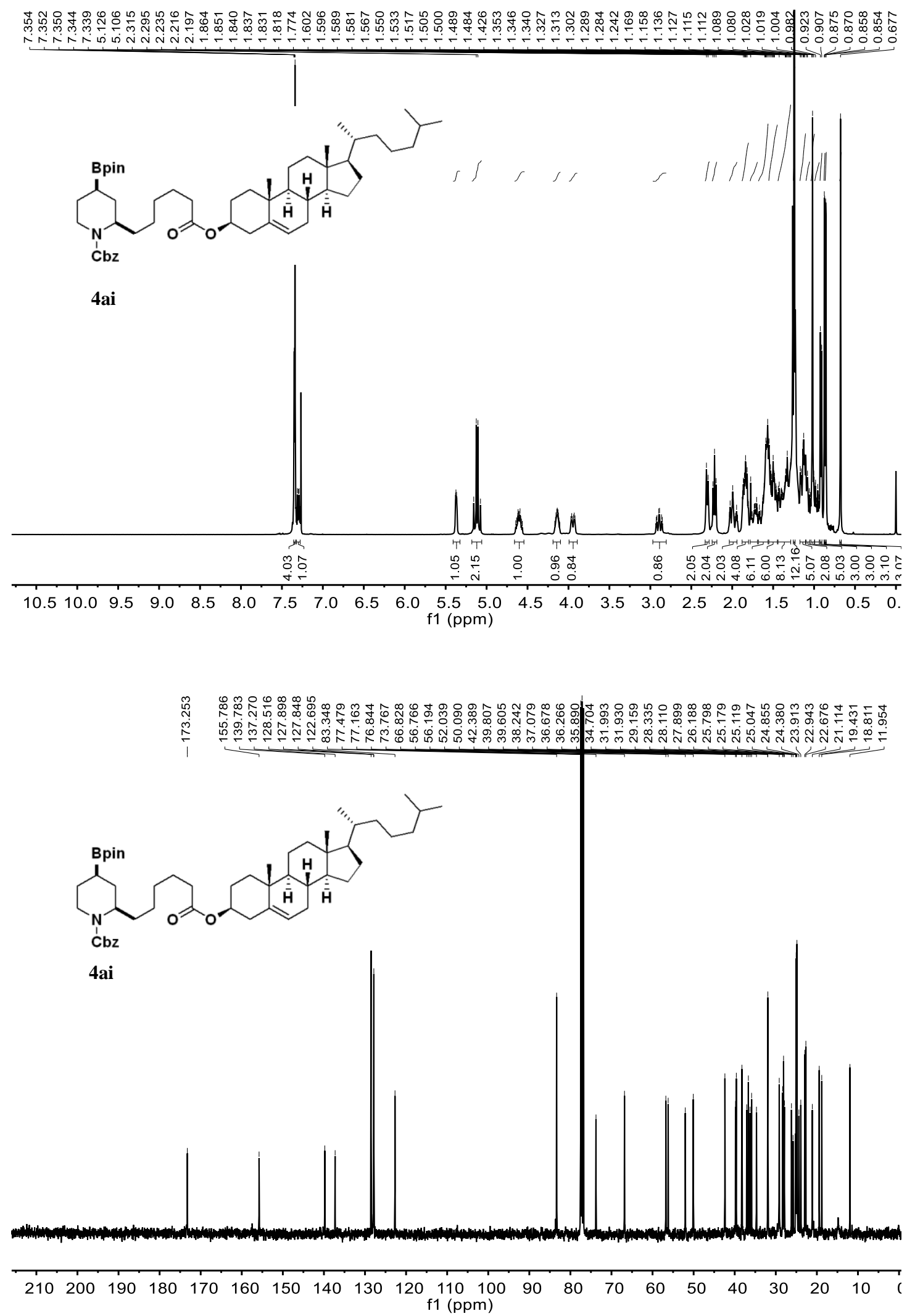


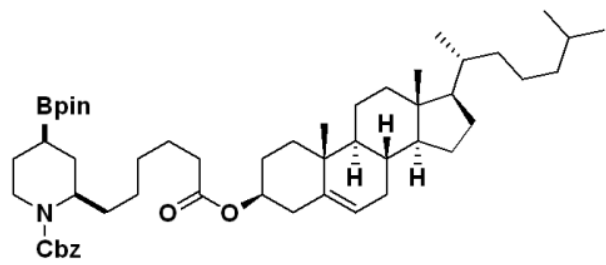

4ai
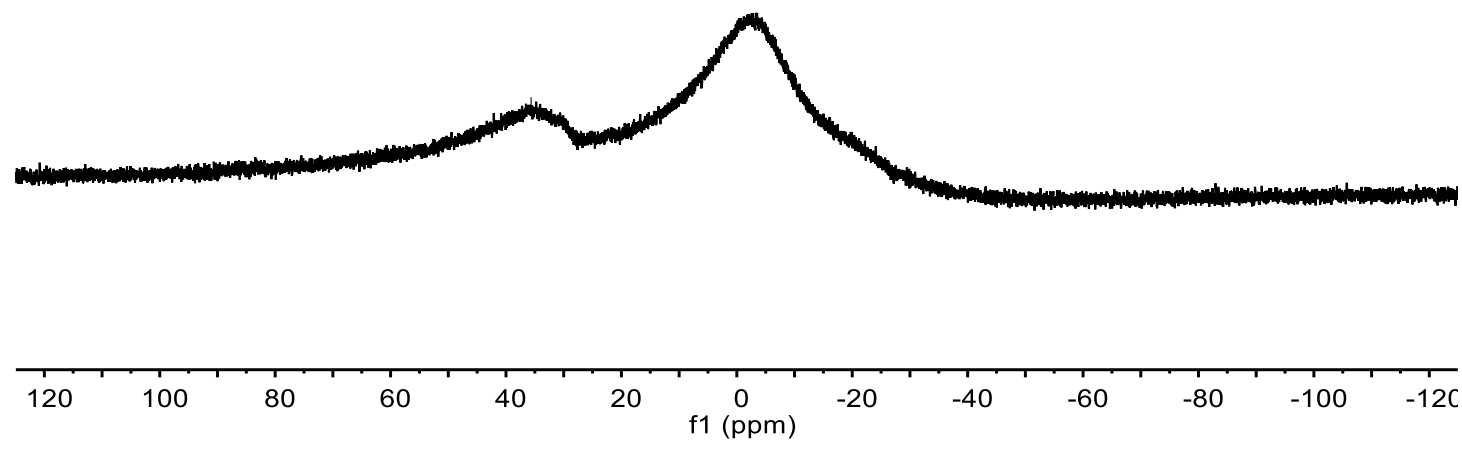

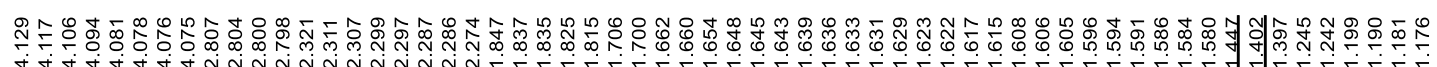

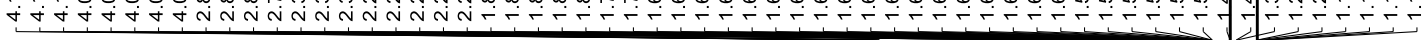<smiles>CCOC(=O)CCCC1CC([Pb](C)(C)C)CCN1C(=O)OCc1ccccc1</smiles>

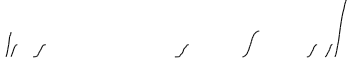

4aj

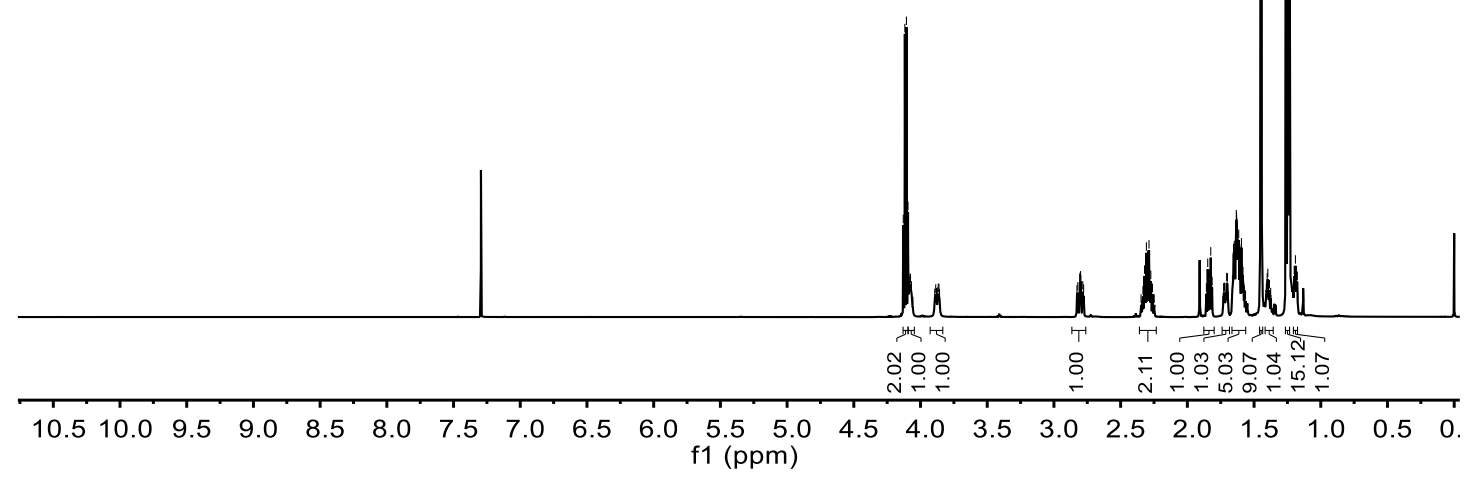




$$
\begin{aligned}
& 1
\end{aligned}
$$

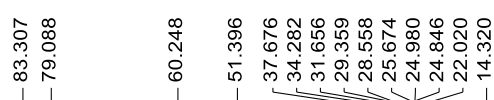
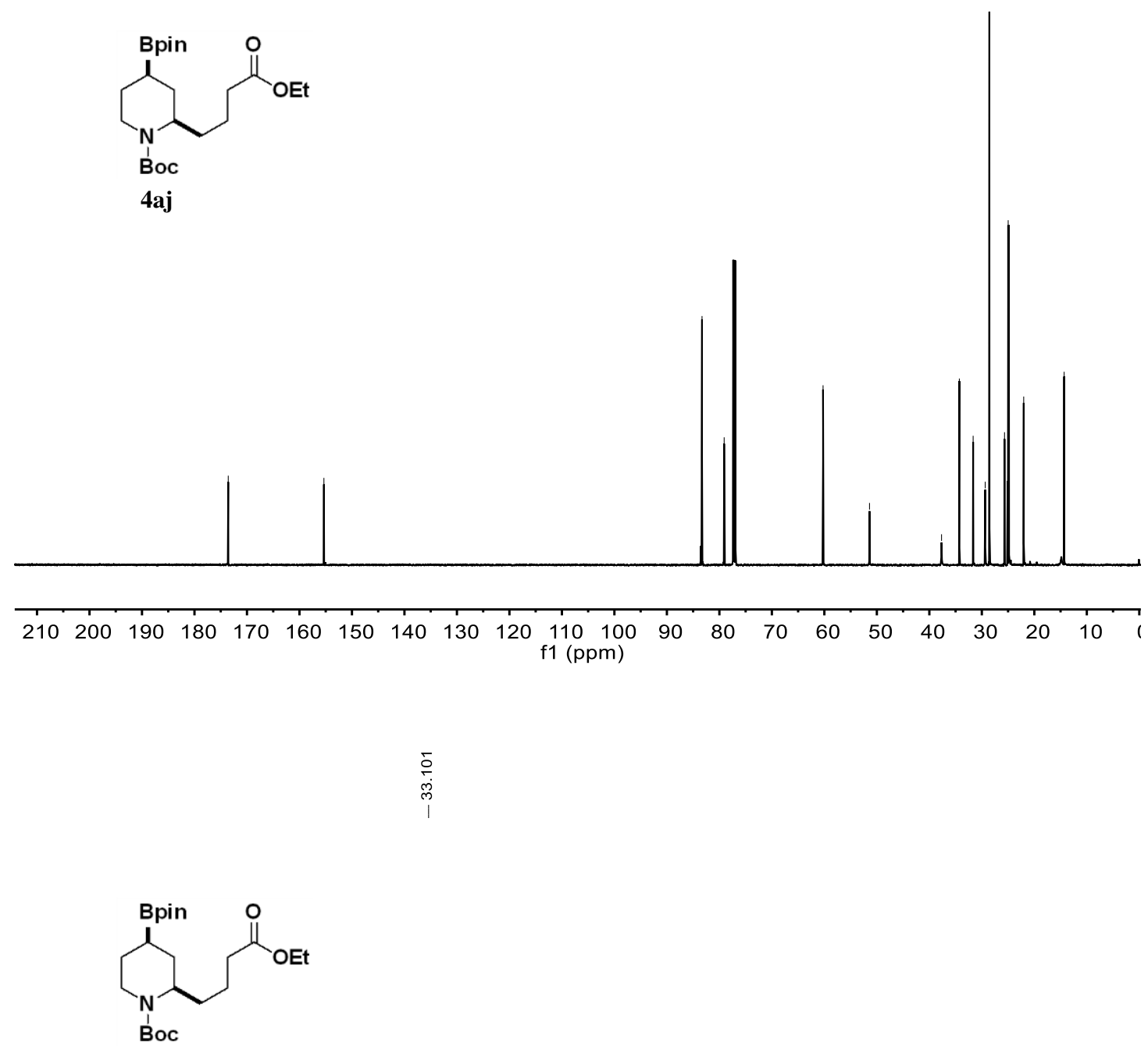

4aj
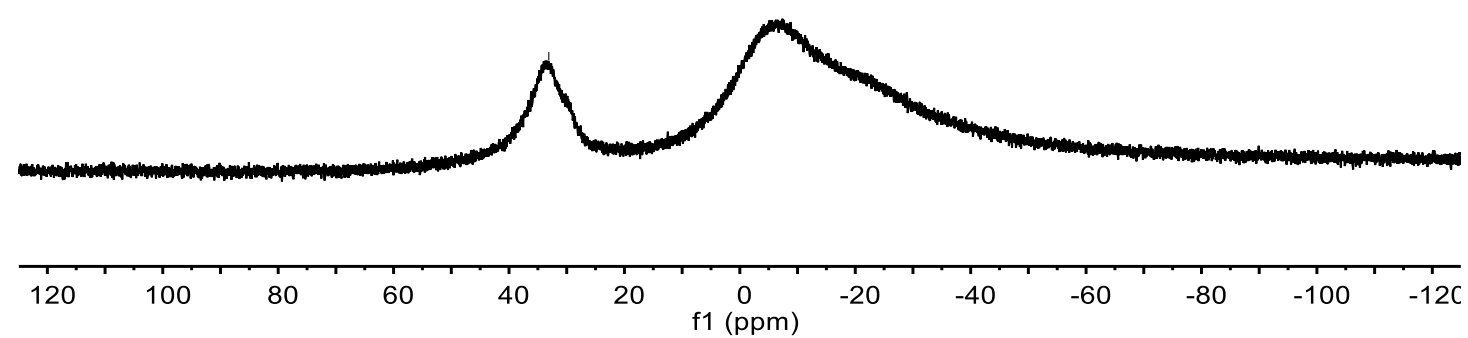


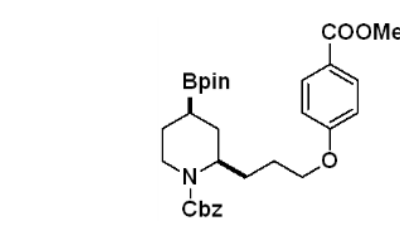

4ak
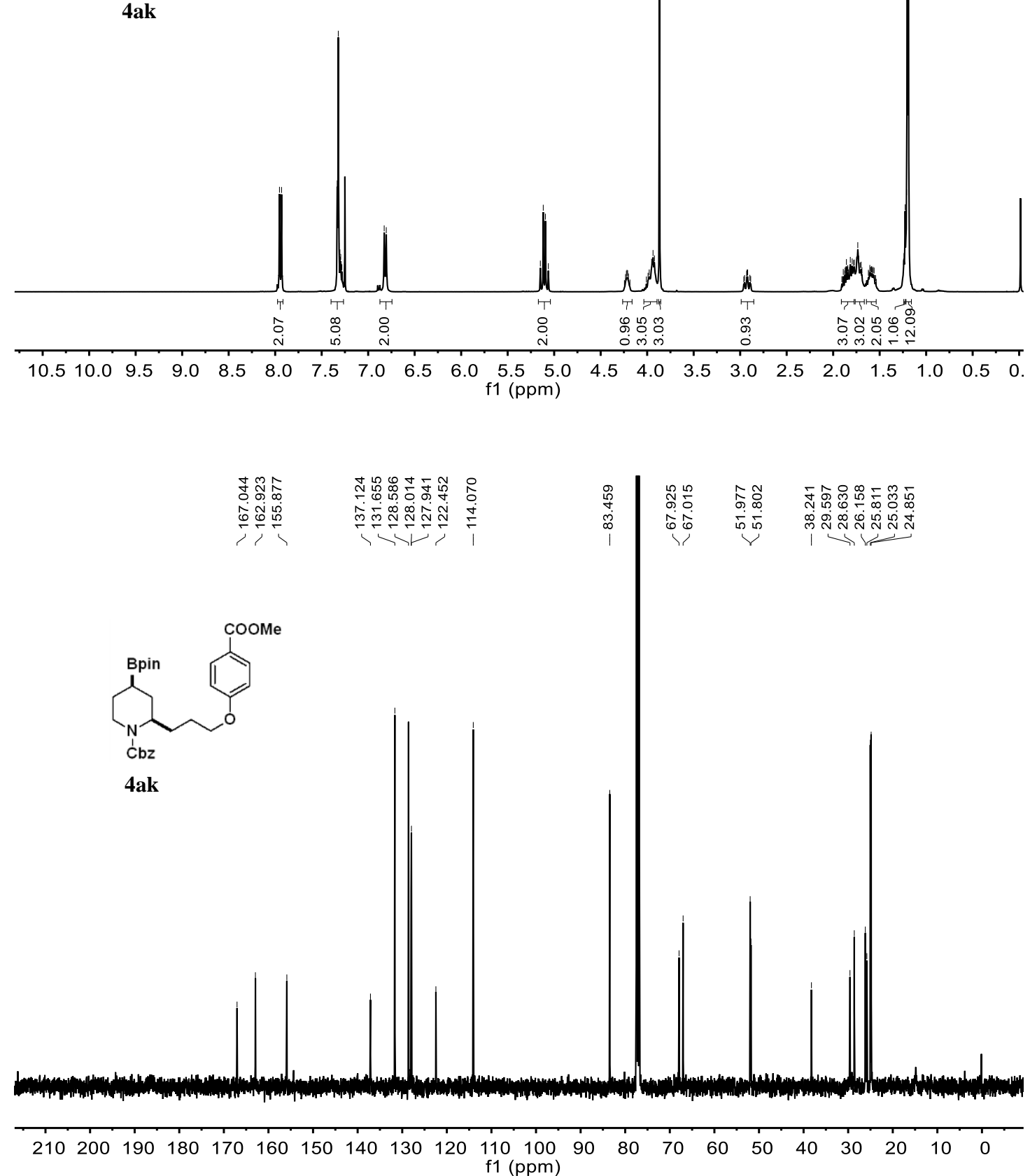


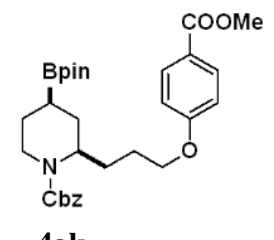

4ak

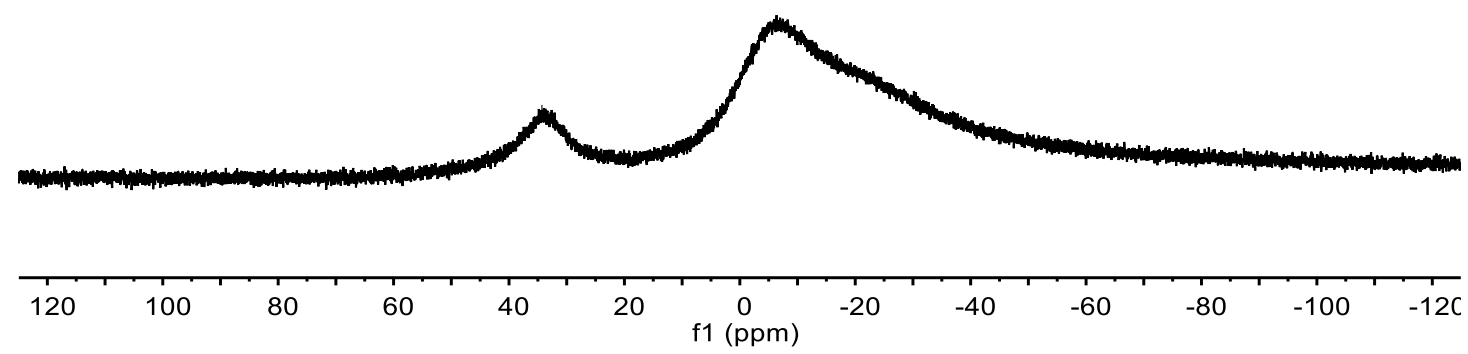

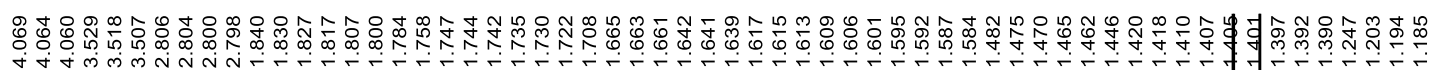<smiles>O=C(OC(=O)N1CCC(Br)CC1CCCCl)c1ccccc1</smiles>

4al

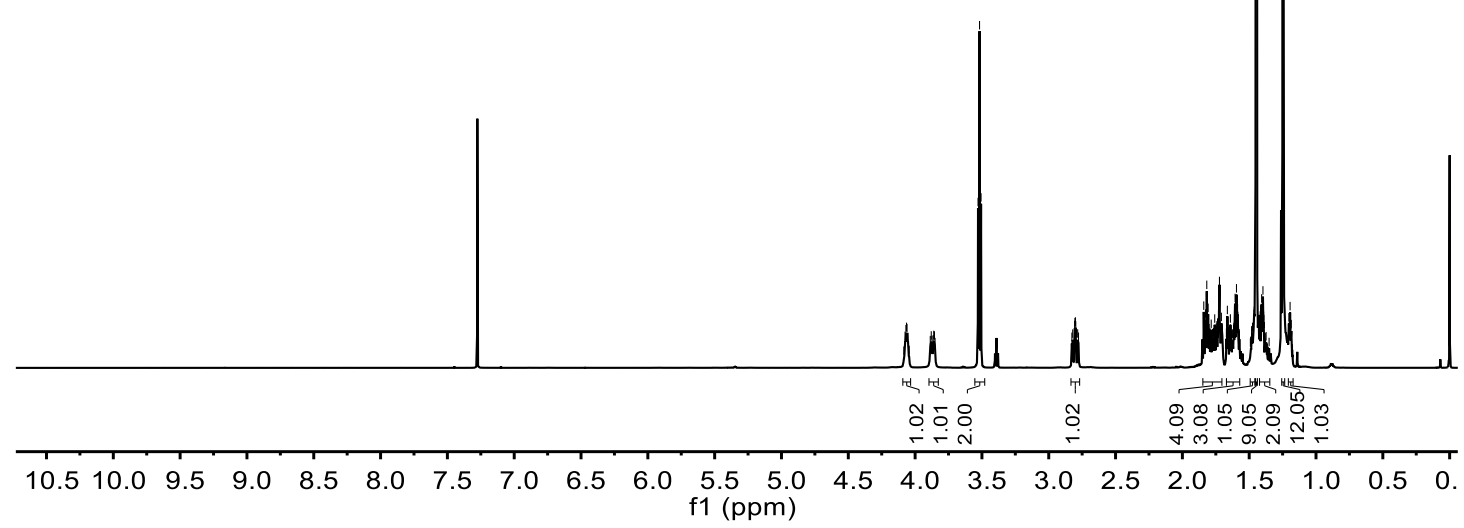



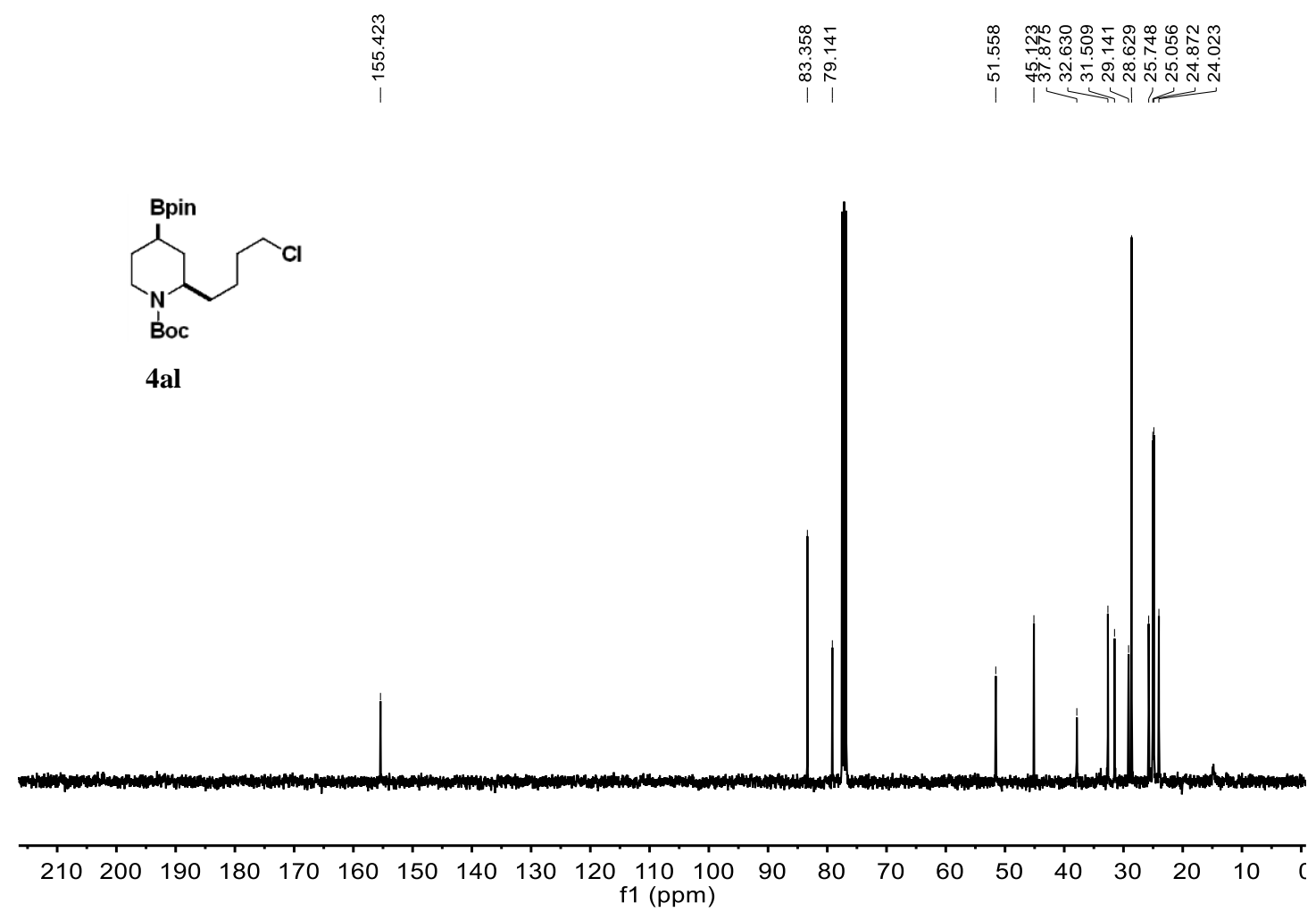

$\frac{\hat{\theta}}{\ddot{m}}$

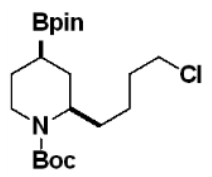

4al

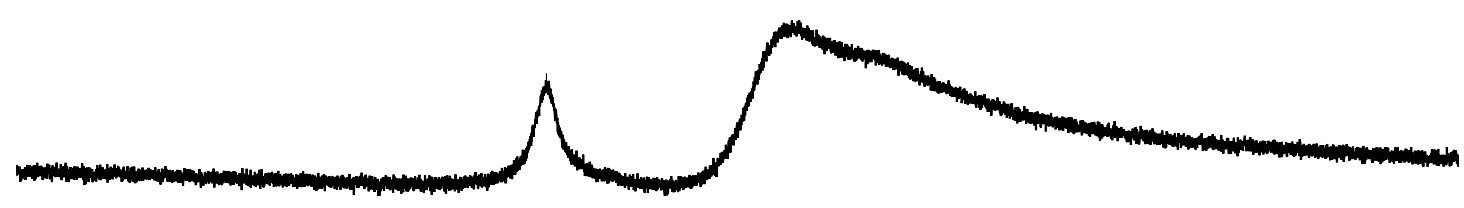

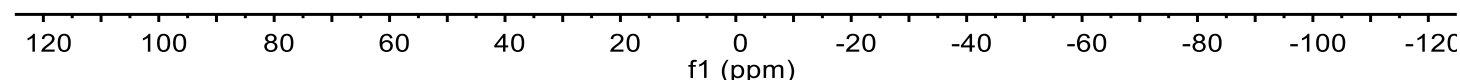



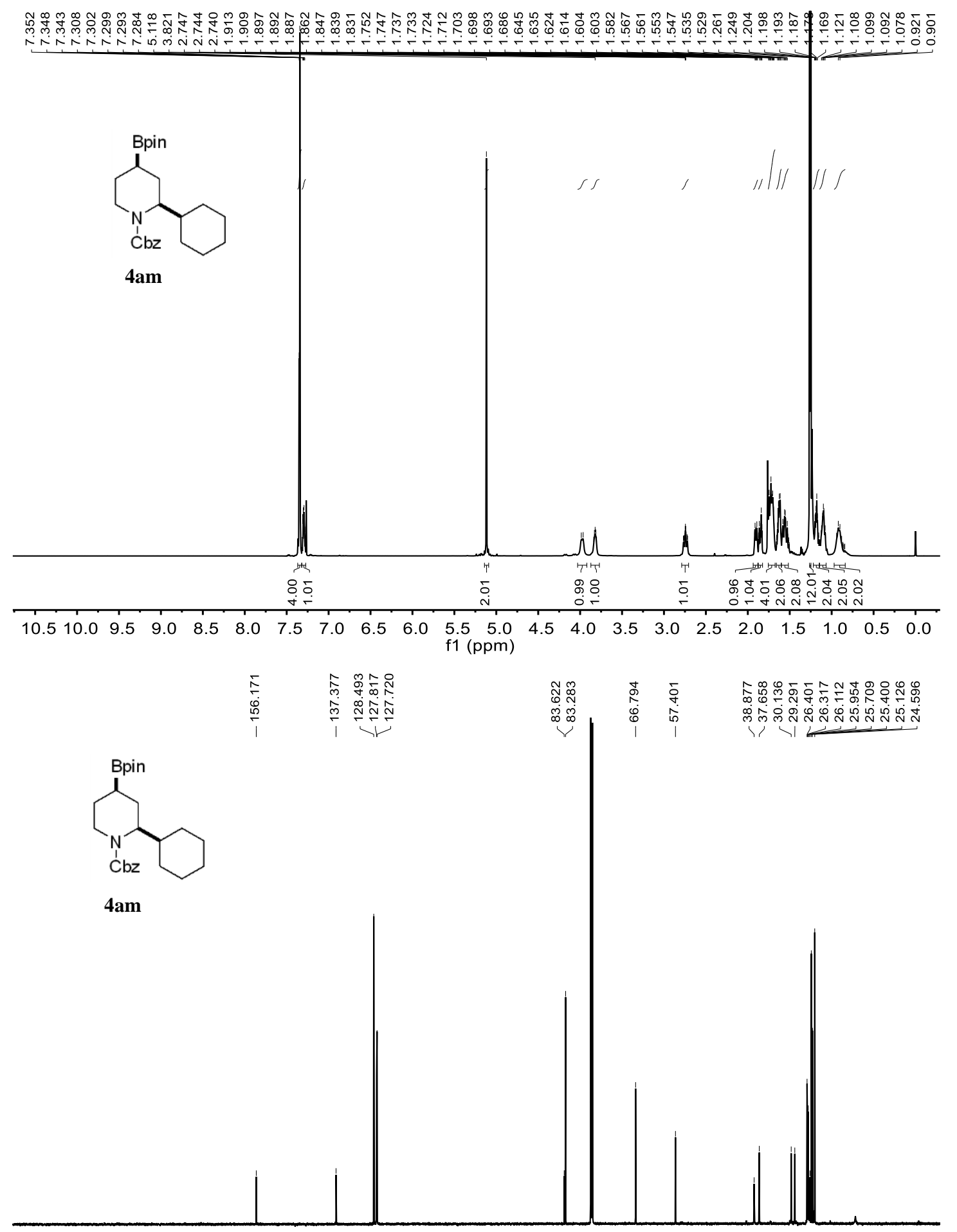

$\begin{array}{llllllllllllllllllllllllllllllllllllll}210 & 200 & 190 & 180 & 170 & 160 & 150 & 140 & 130 & 120 & 110 & 100 & 90 & 80 & 70 & 60 & 50 & 40 & 30 & 20 & 10 & 0\end{array}$ f1 (ppm) 


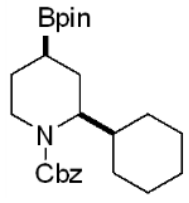

4am
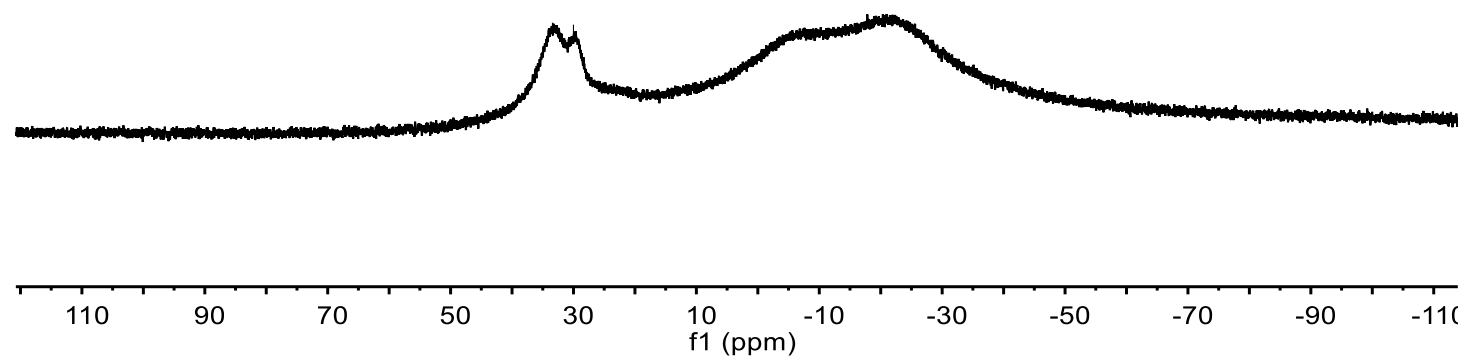

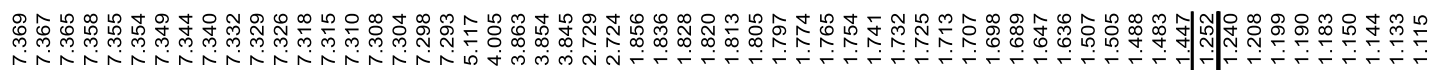

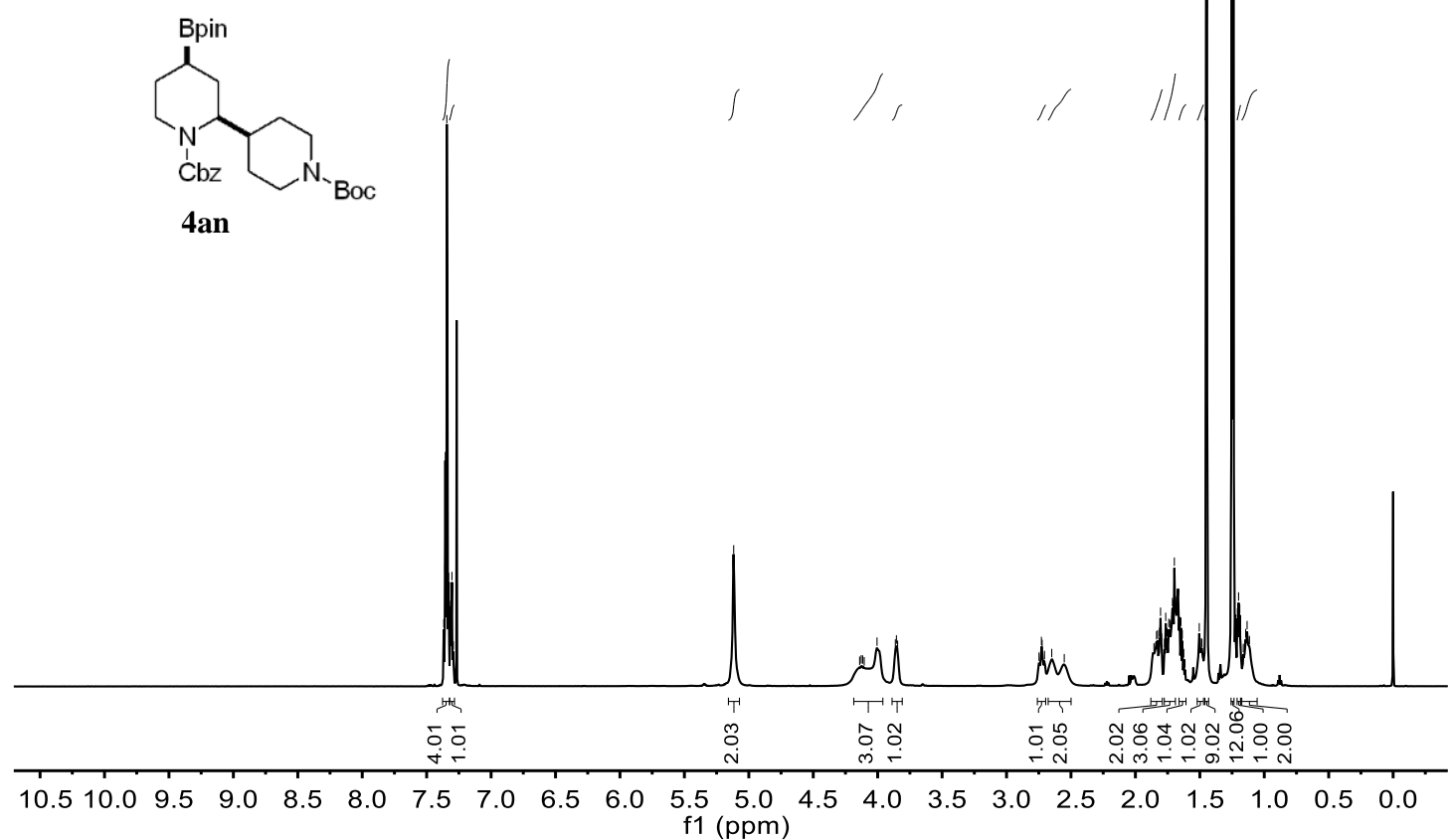



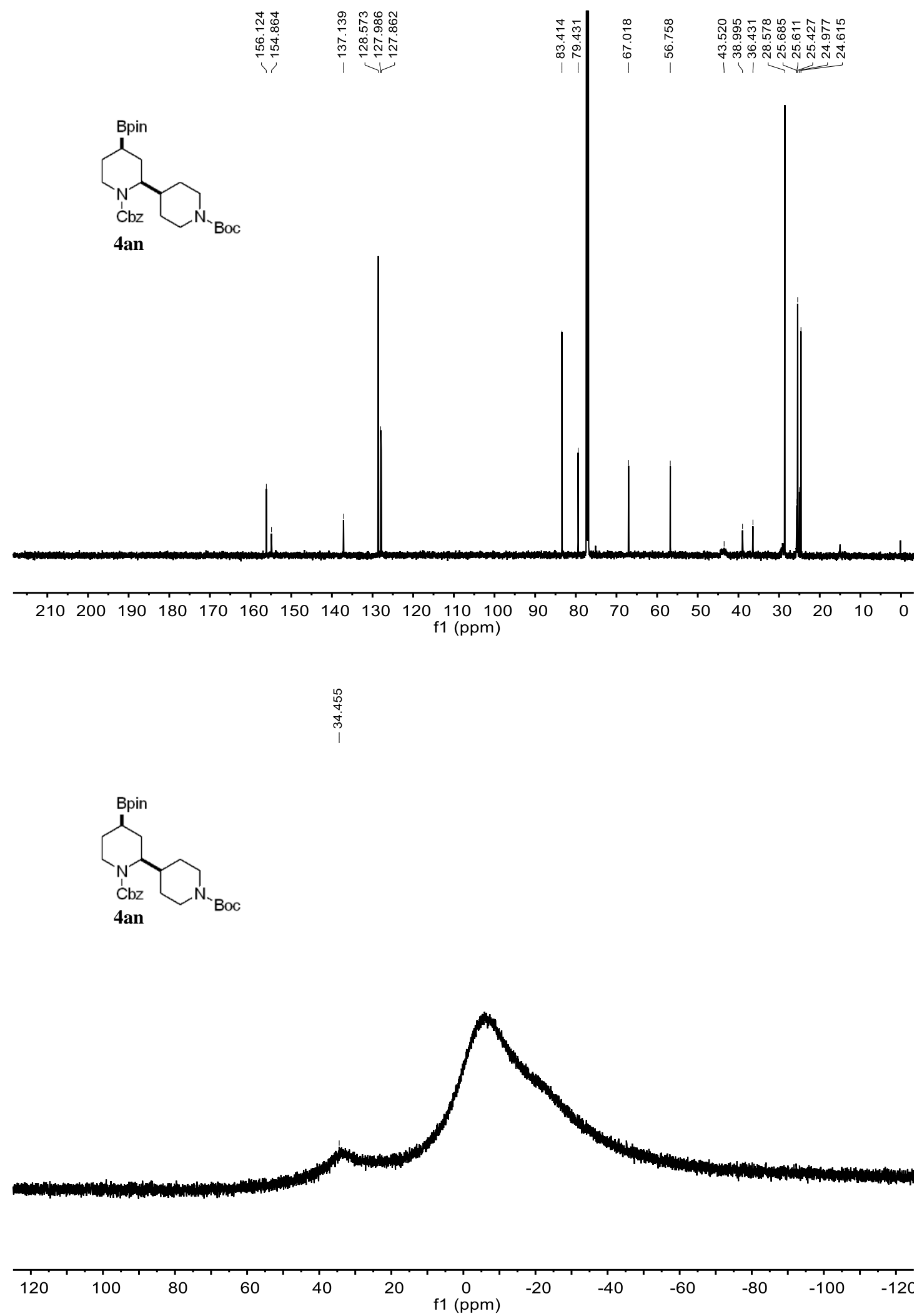

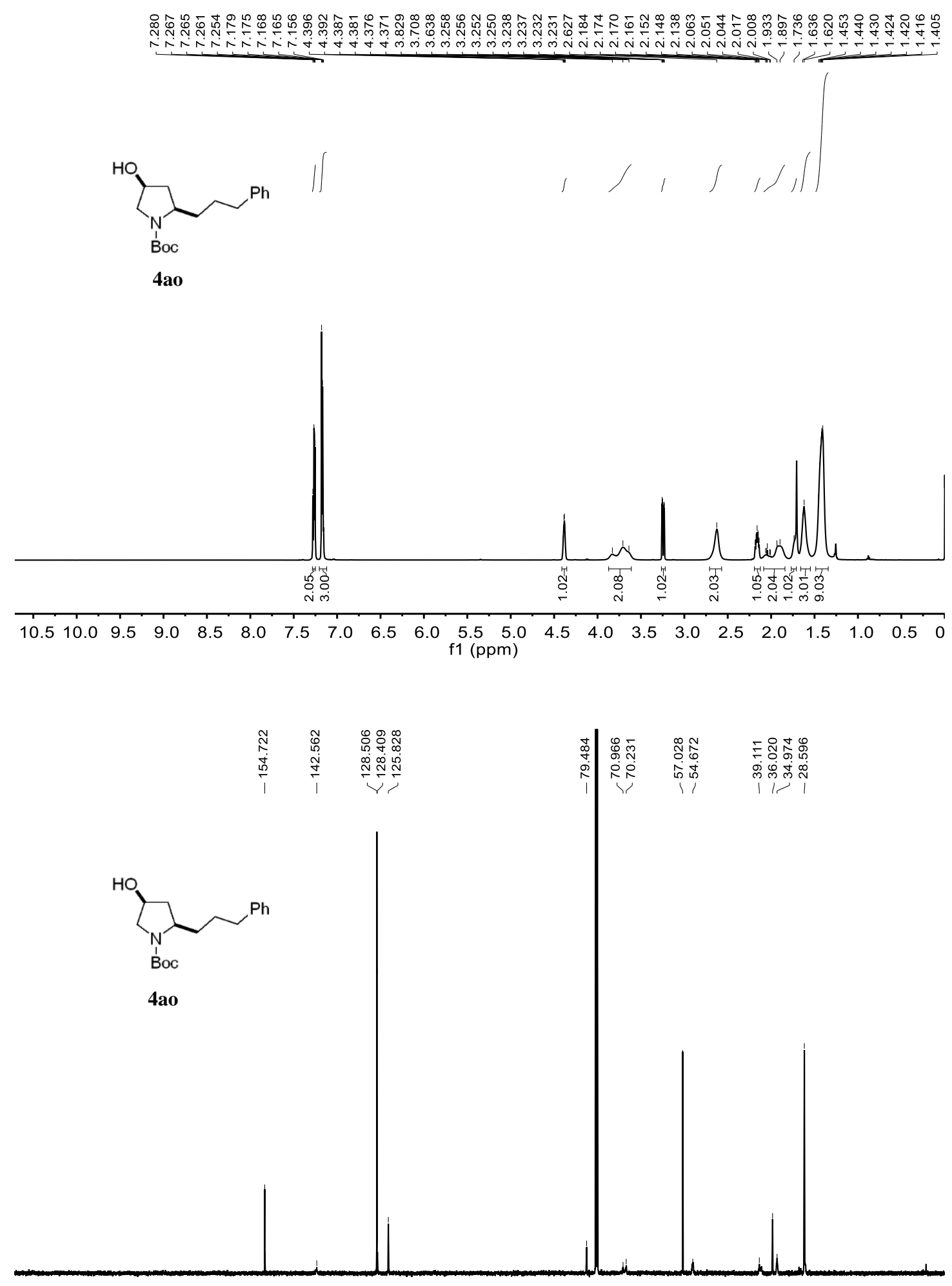

$\begin{array}{llllllllllllllllllllll}210 & 200 & 190 & 180 & 170 & 160 & 150 & 140 & 130 & 120 & 110 & 100 & 90 & 80 & 70 & 60 & 50 & 40 & 30 & 20 & 10 & 0\end{array}$ f1 (ppm) 

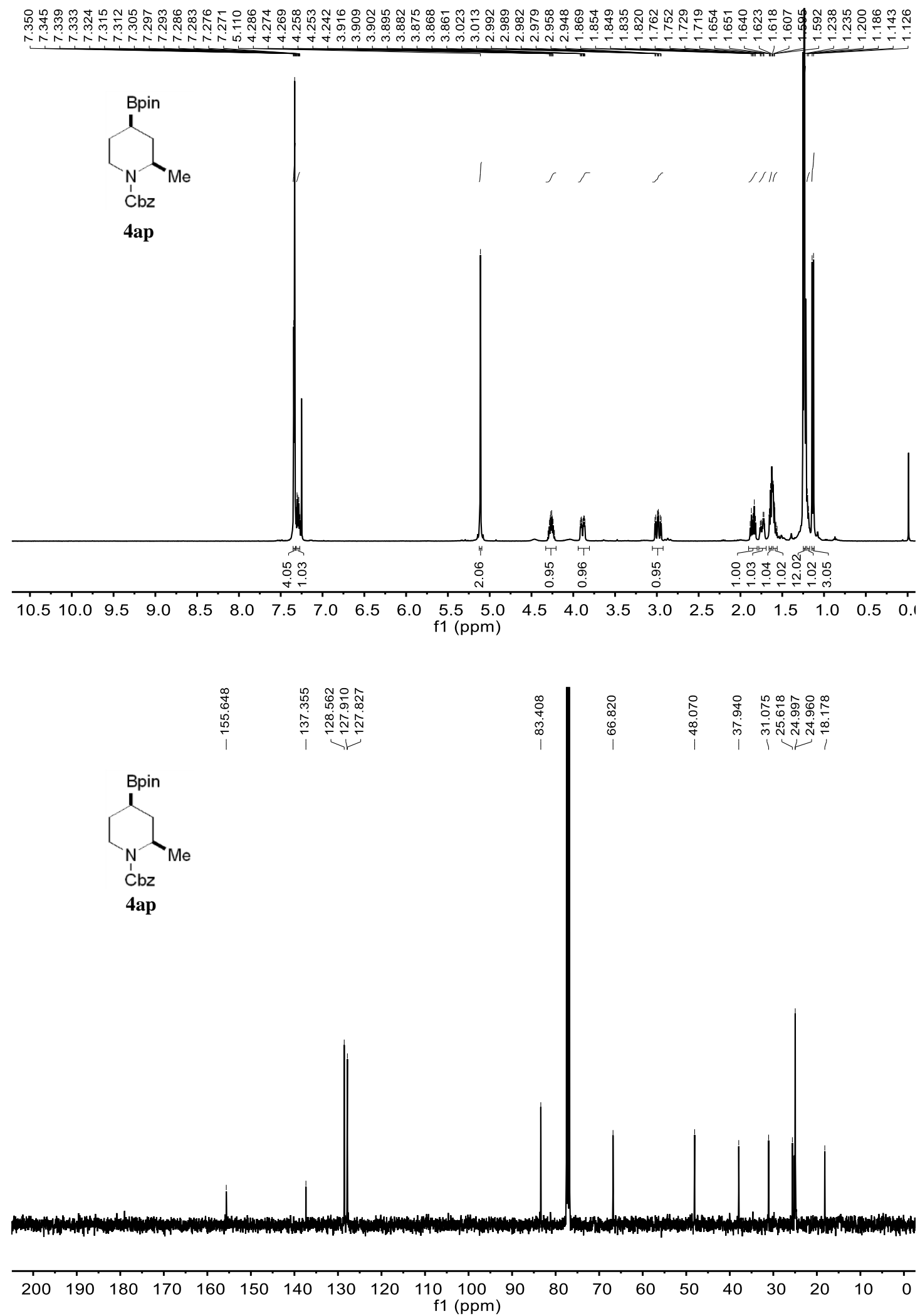


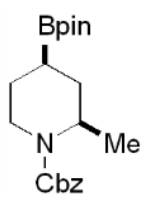

4ap
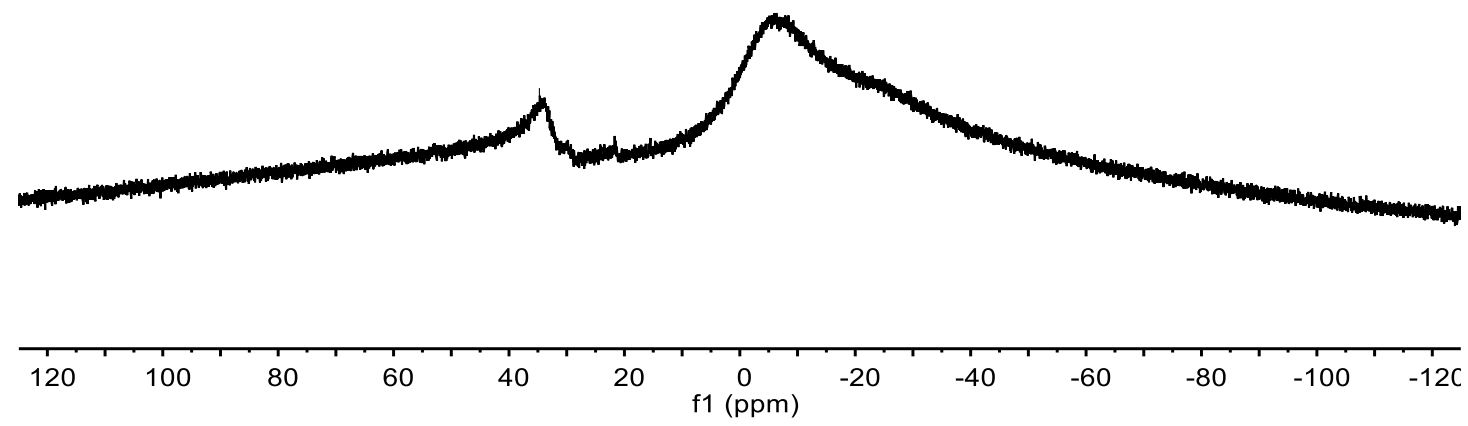

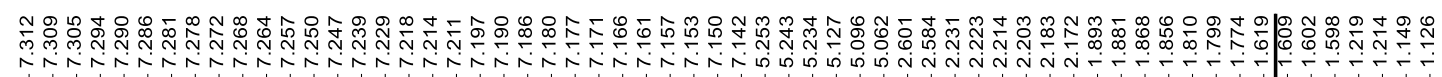

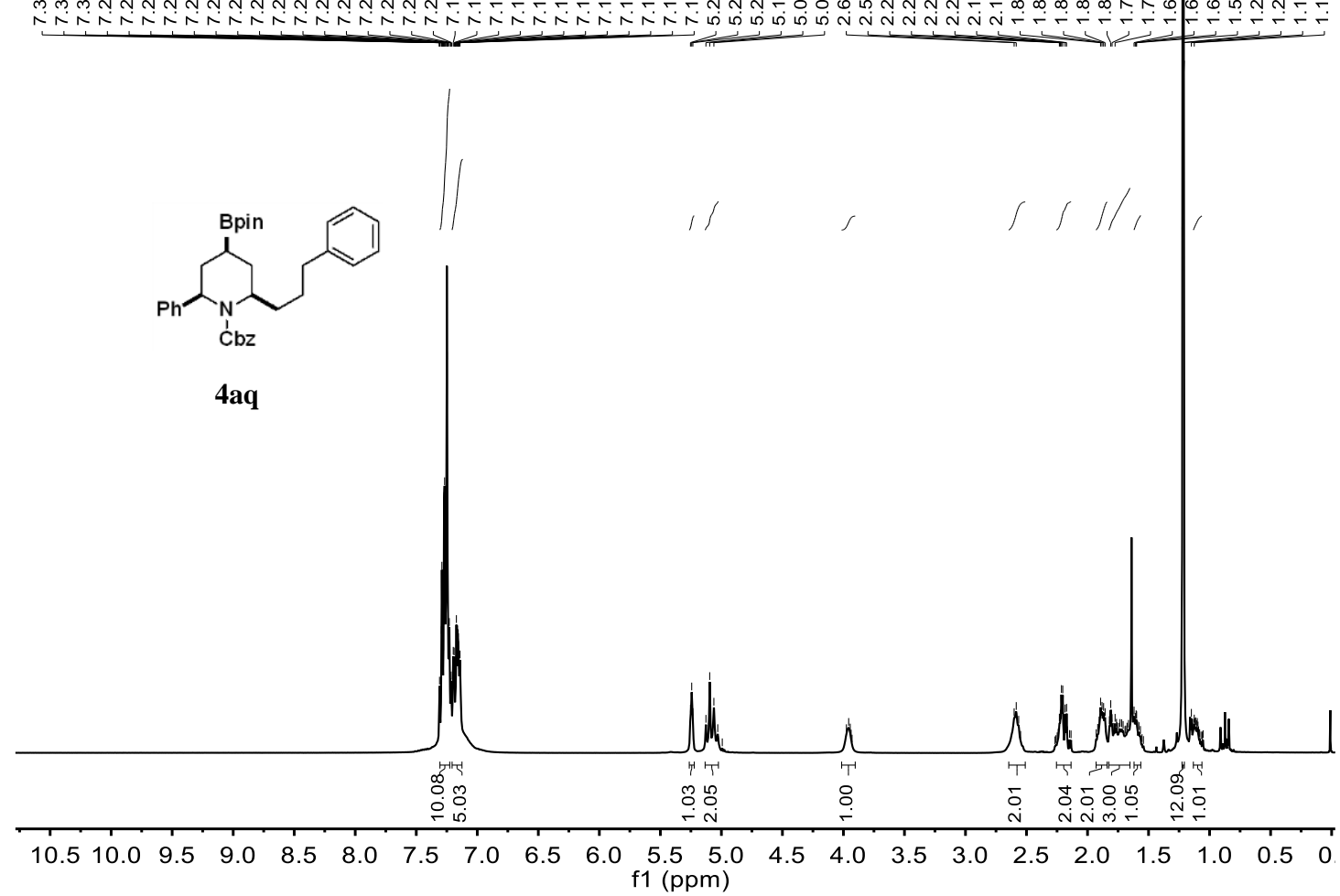



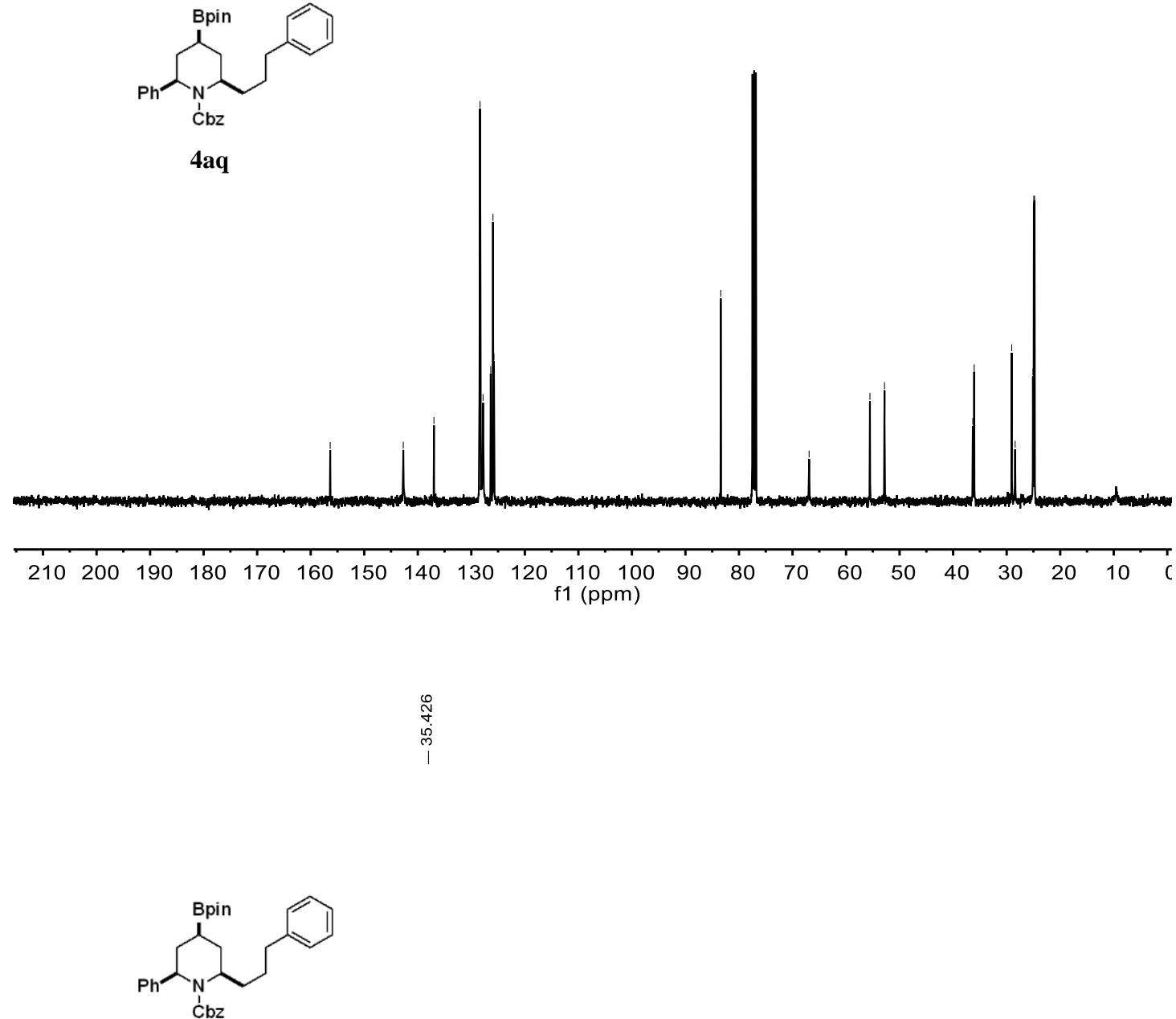

4aq

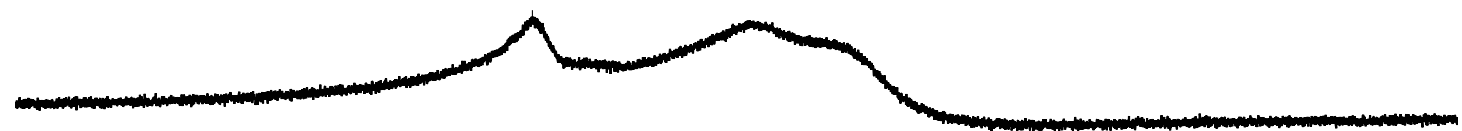

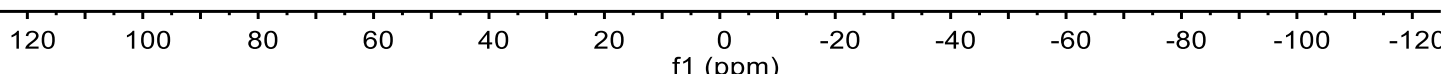



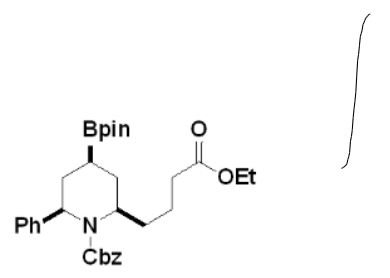

4ar
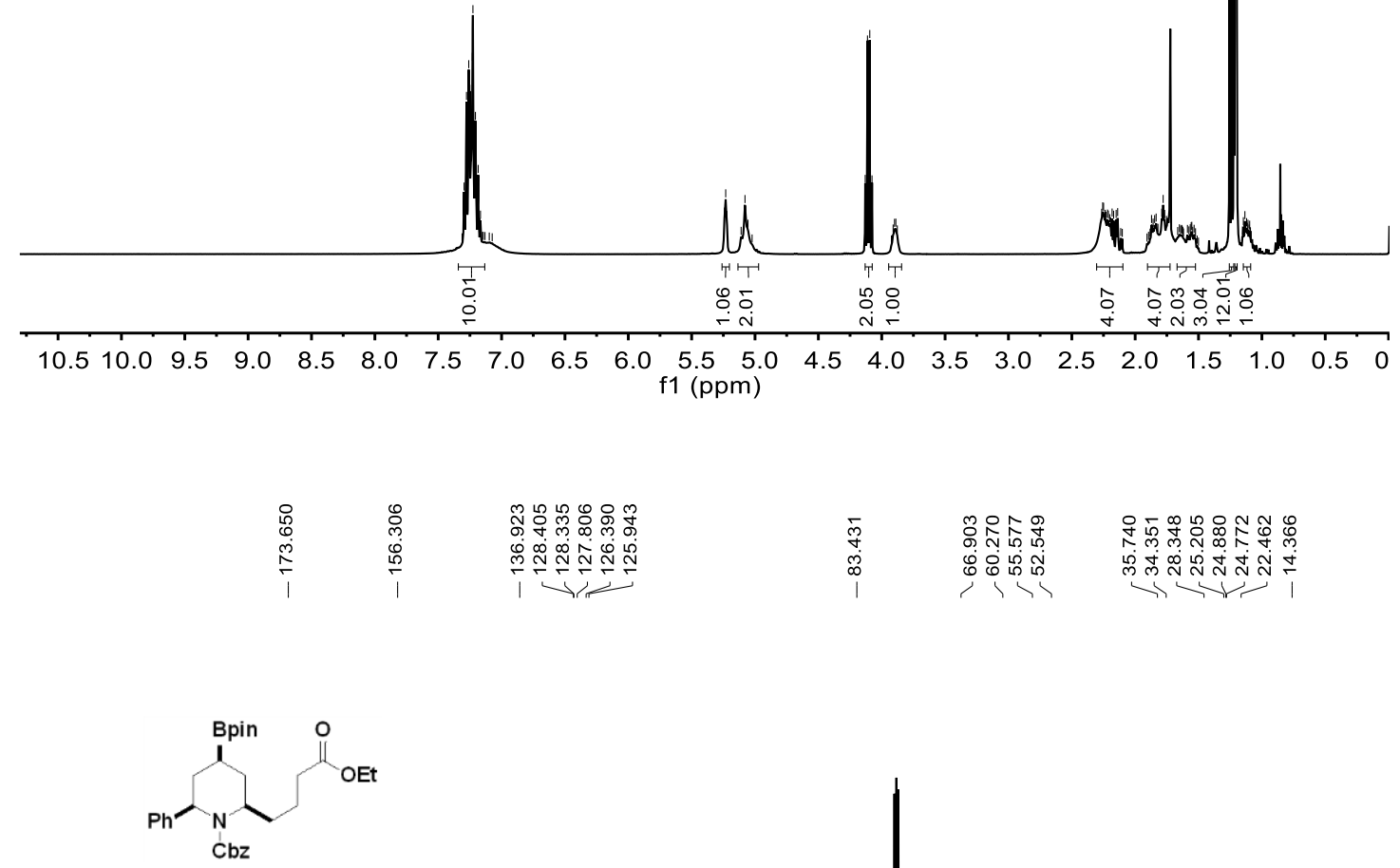

4ar

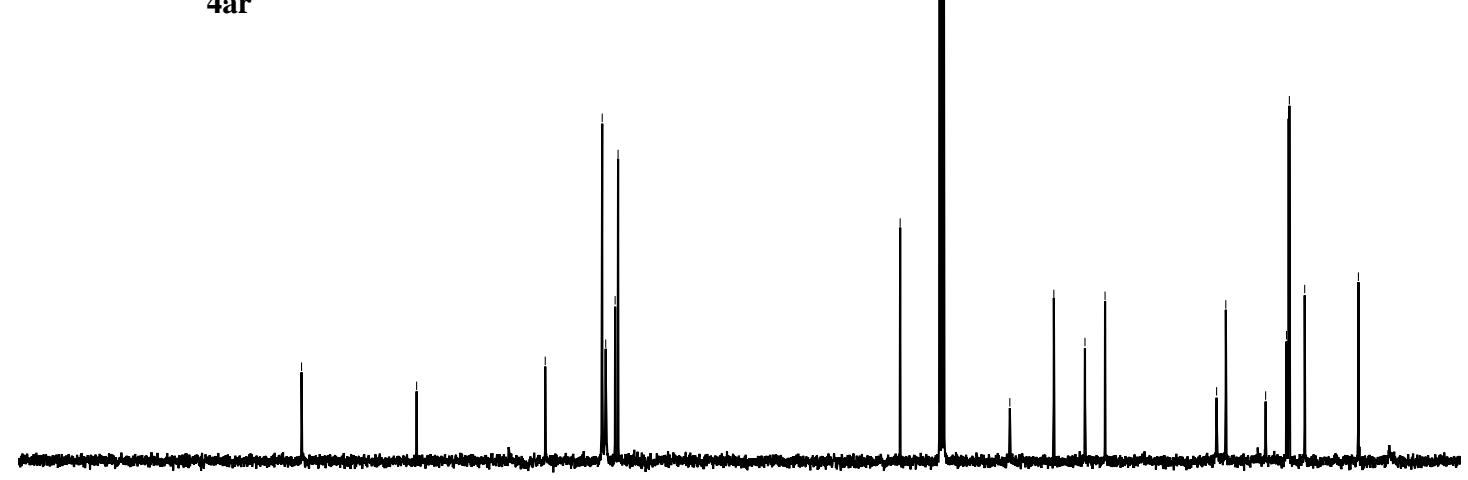

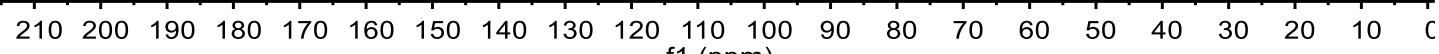
f1 (ppm) 

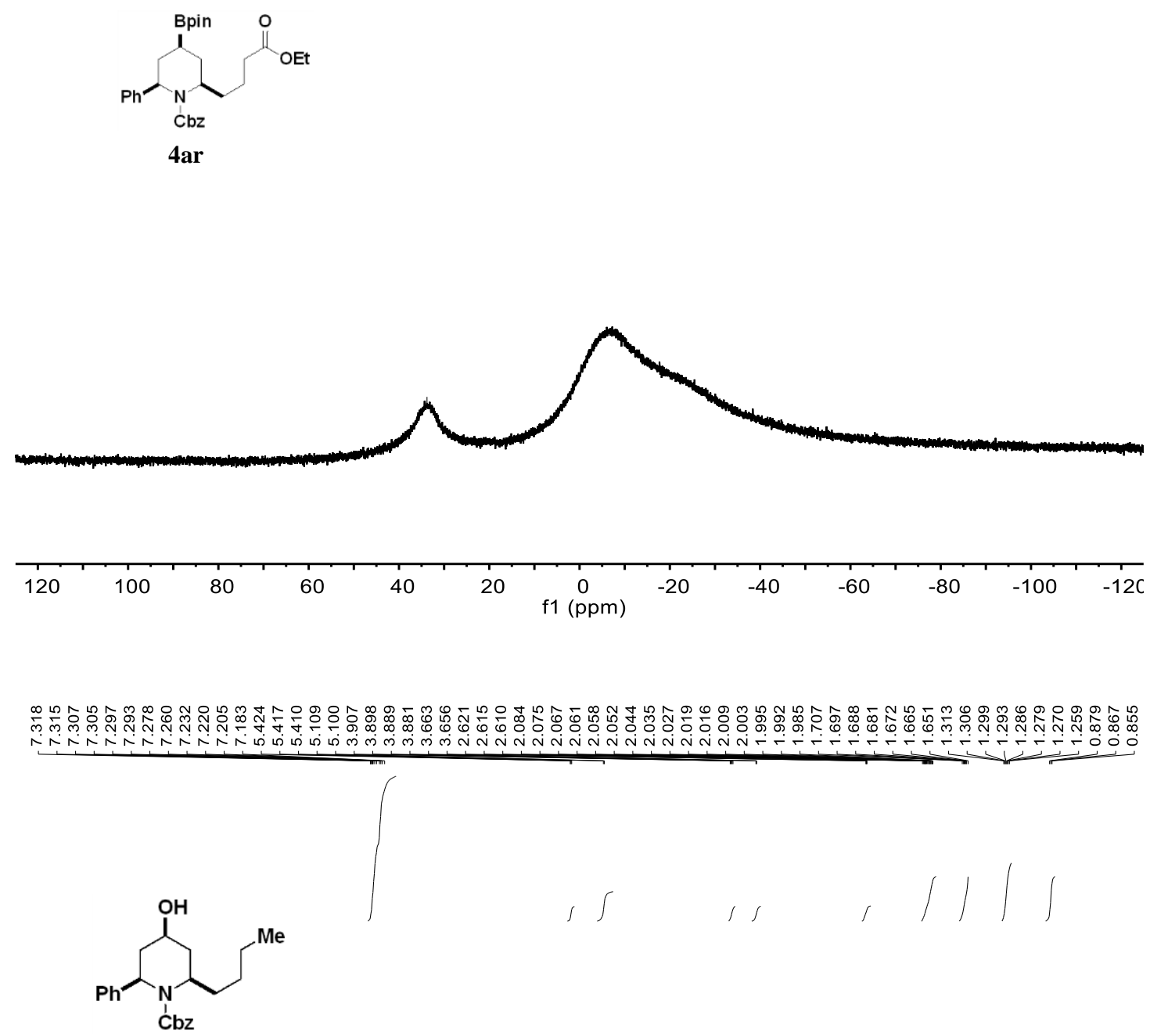

4as

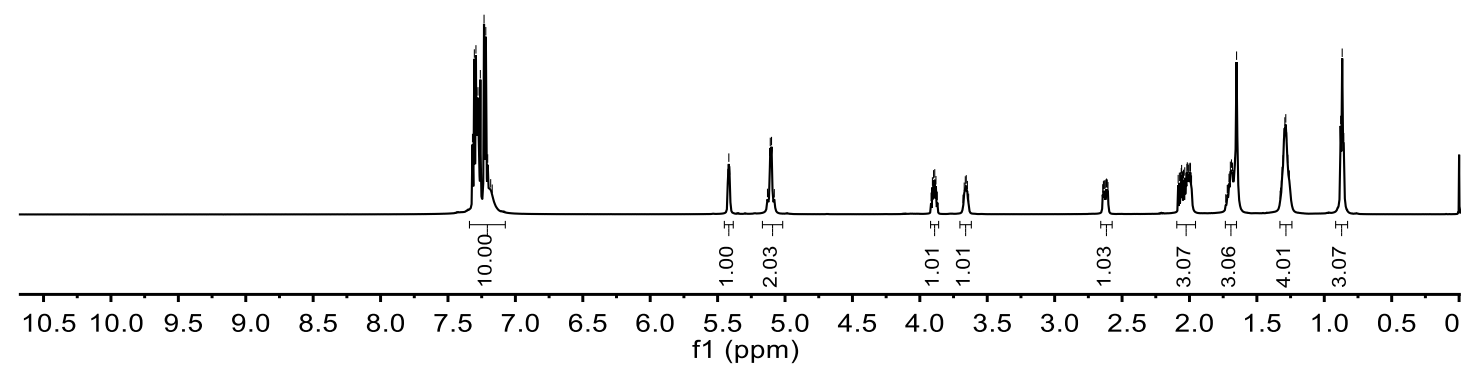




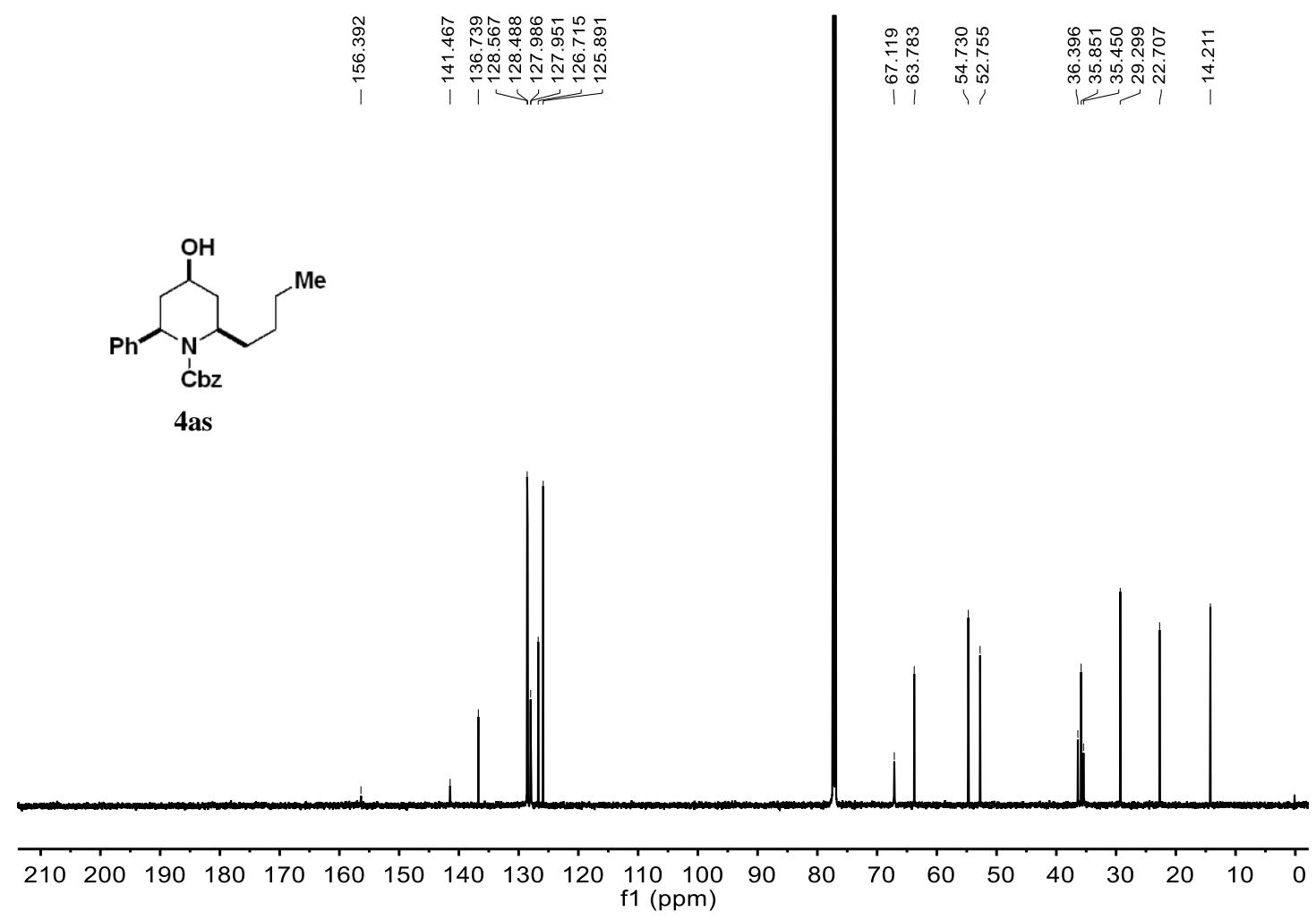

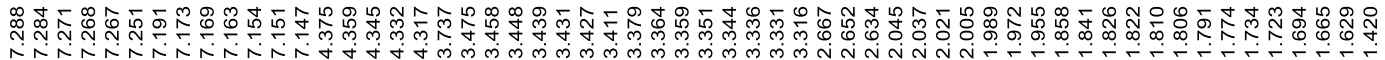
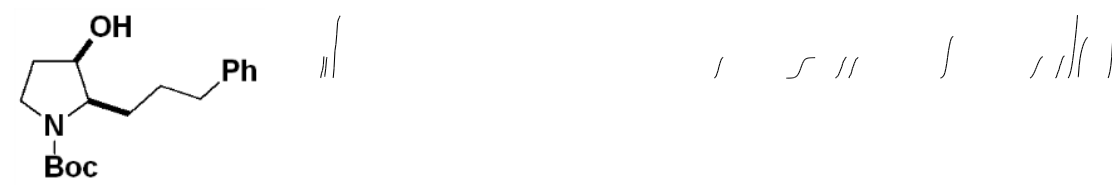

4at

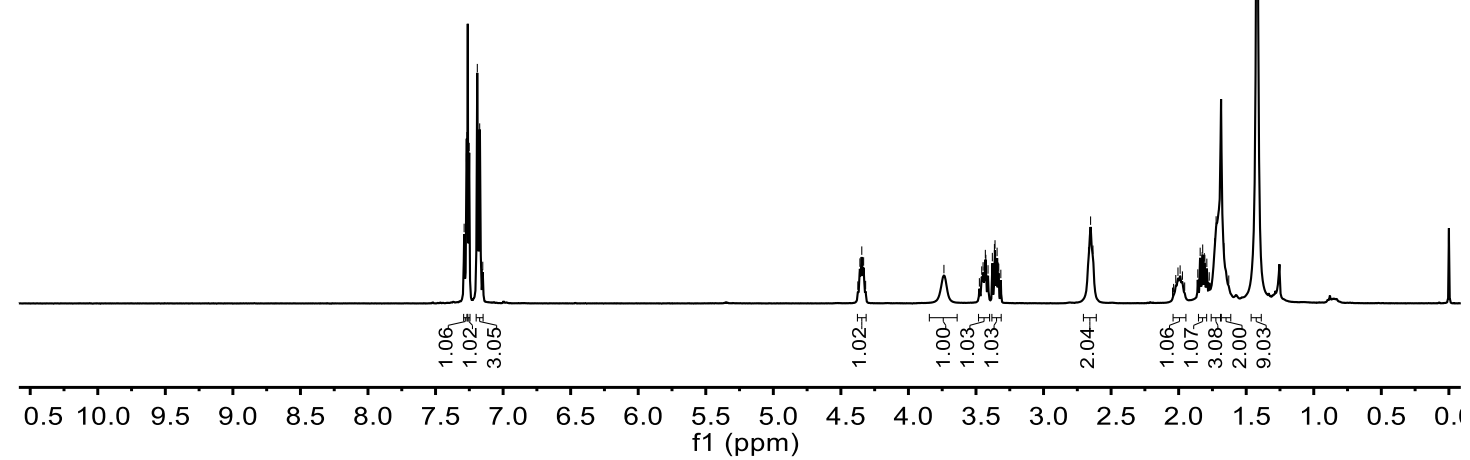




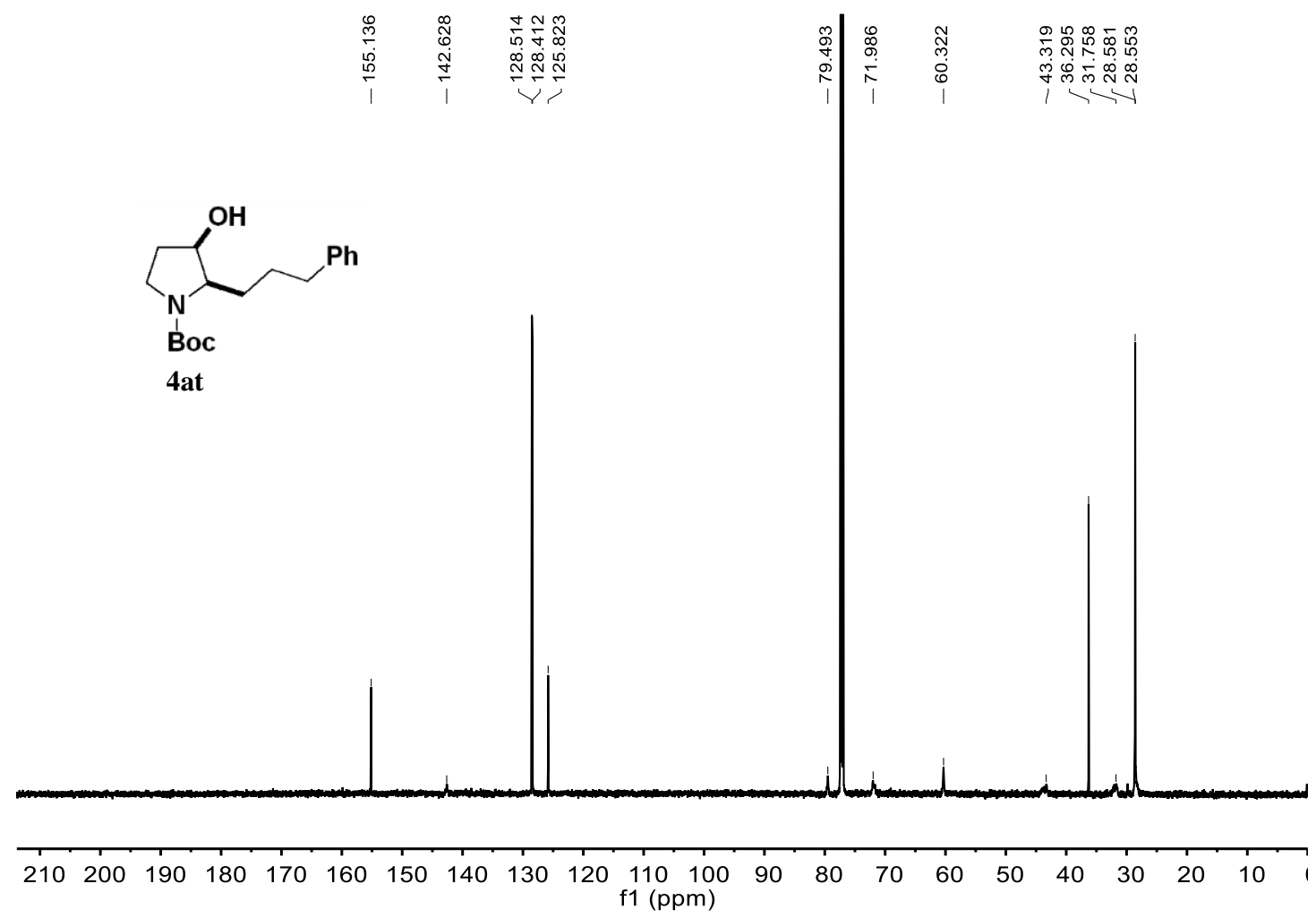

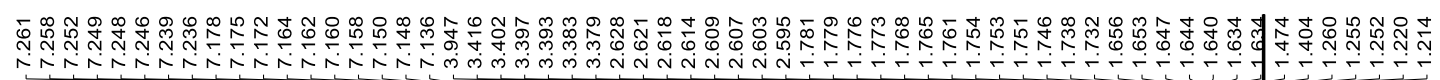

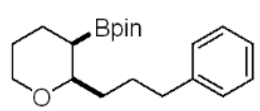

4 au

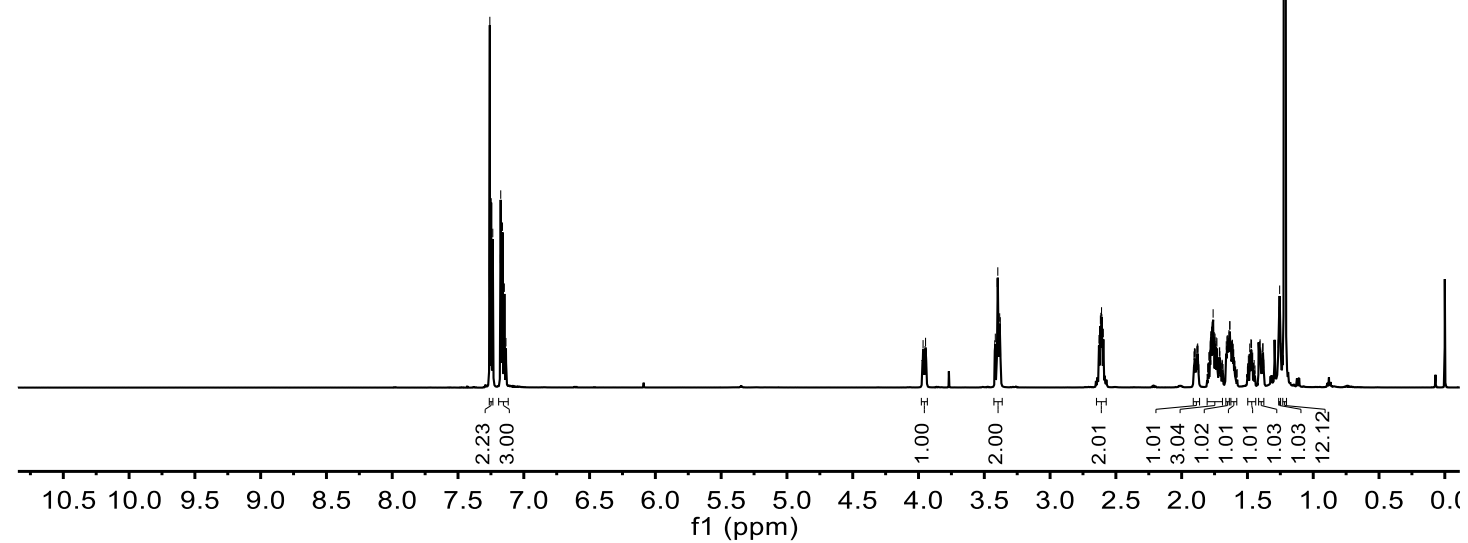



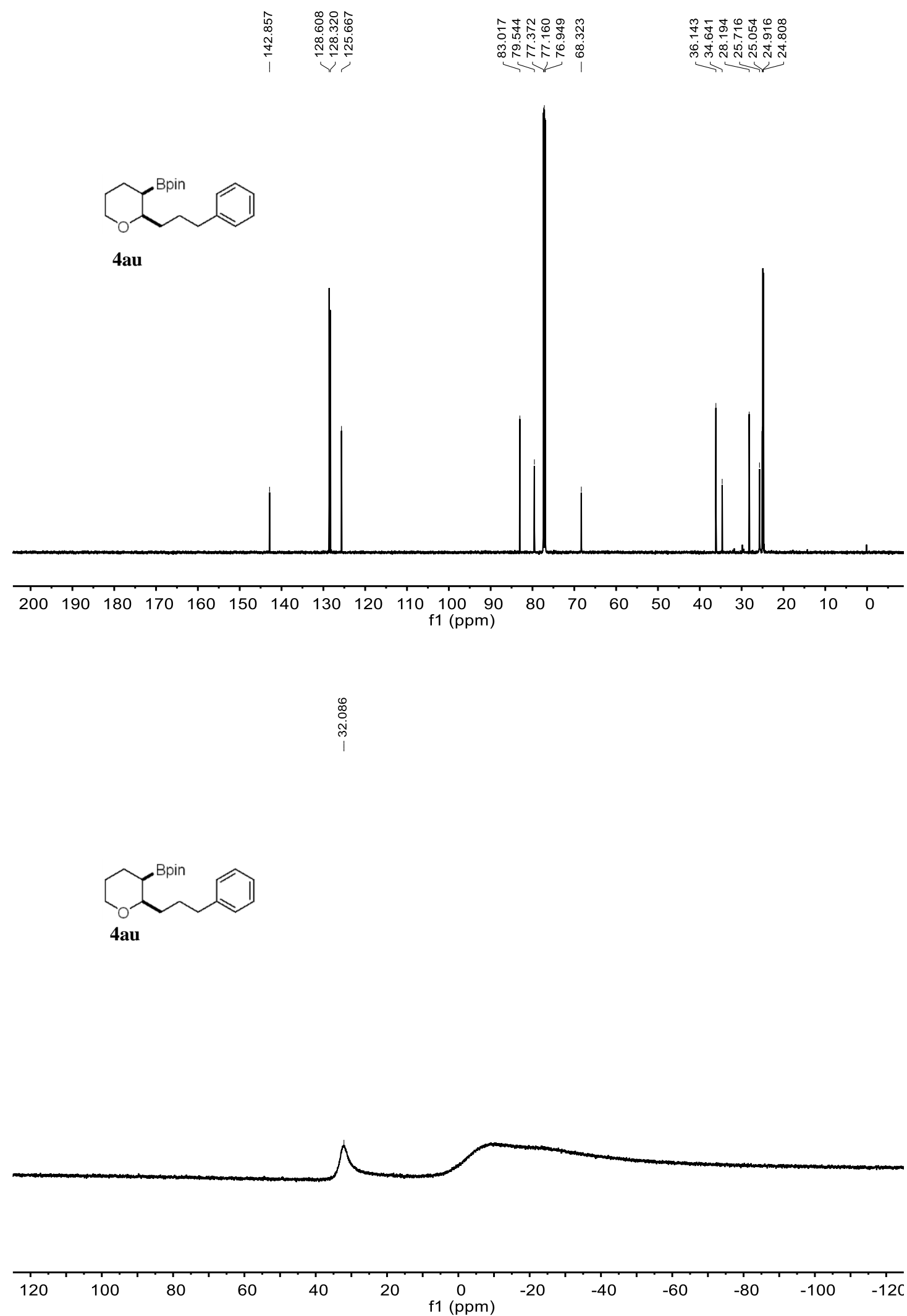

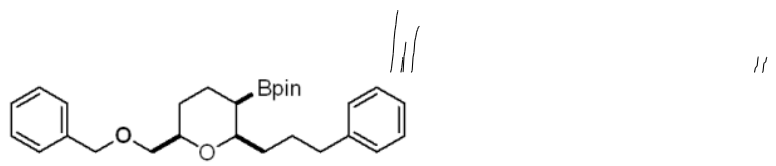

4av

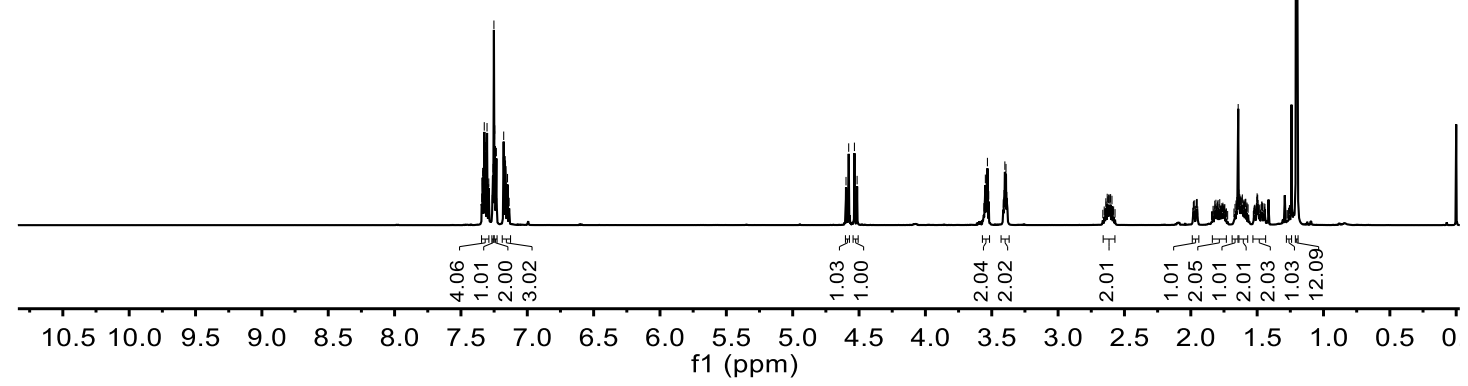

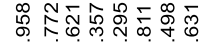

ำ

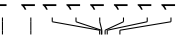

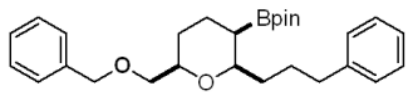

4av

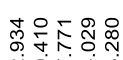

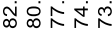

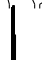

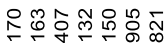

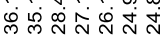

凩然

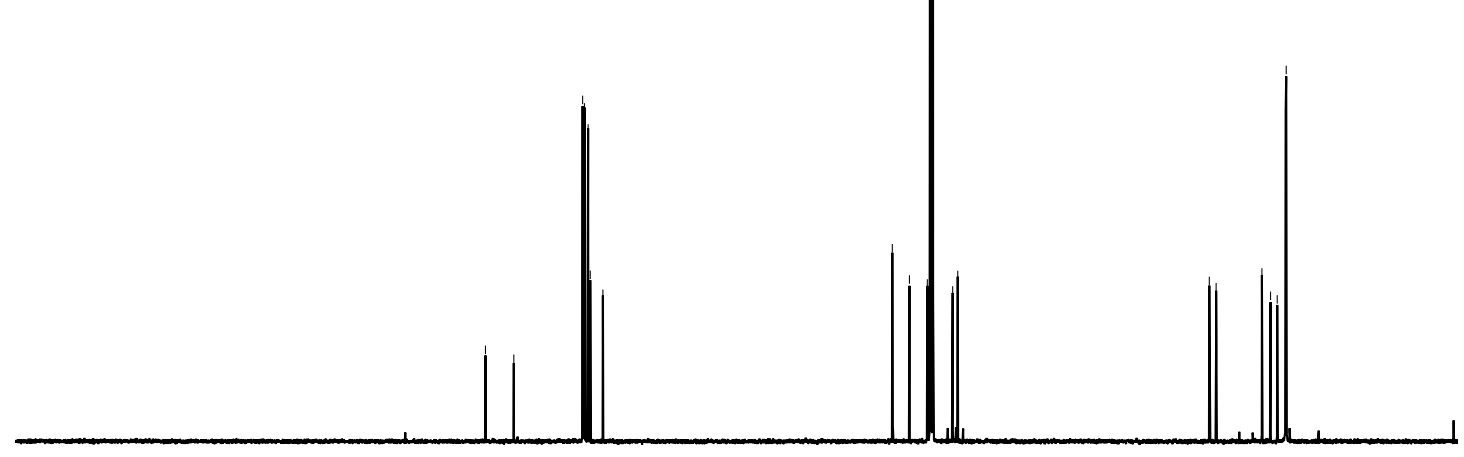

$\begin{array}{lllllllllllllllllllll}210 & 200 & 190 & 180 & 170 & 160 & 150 & 140 & 130 & 120 & 110 & 100 & 90 & 80 & 70 & 60 & 50 & 40 & 30 & 20 & 10\end{array}$ f1 (ppm) 


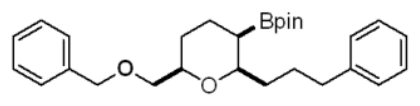

$4 a v$

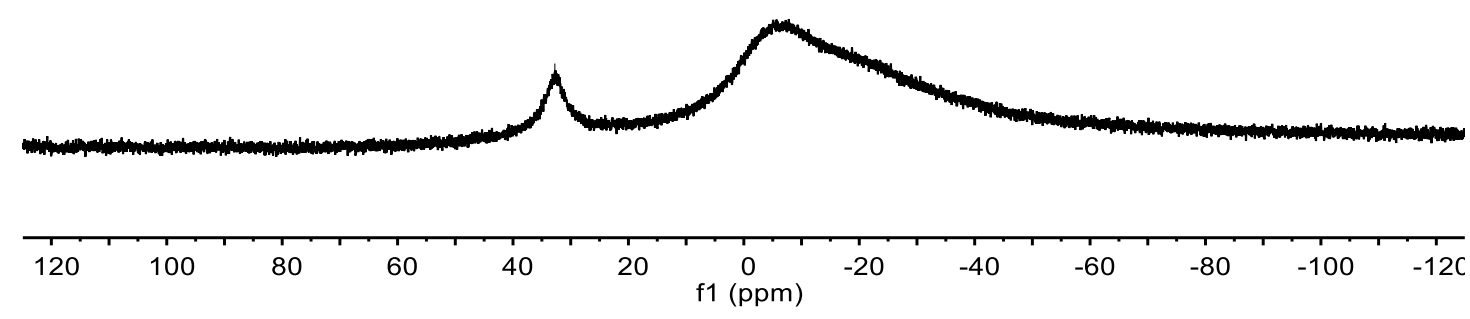

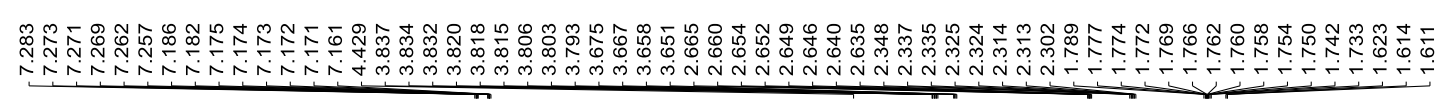
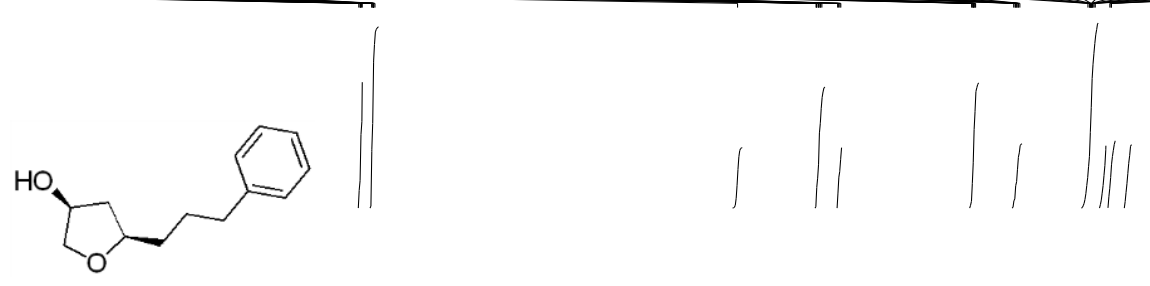

$(3 S, 5 R)-4 a$

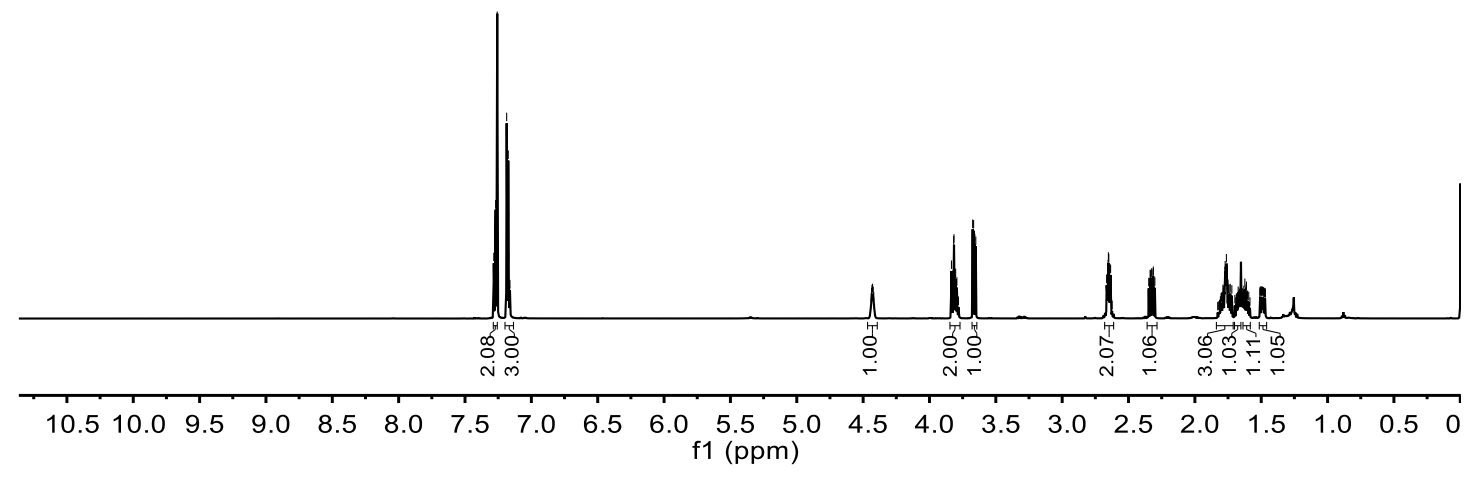




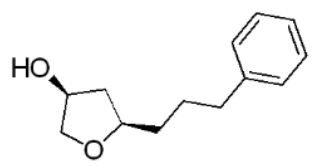

$(3 S, 5 R)-4 a$

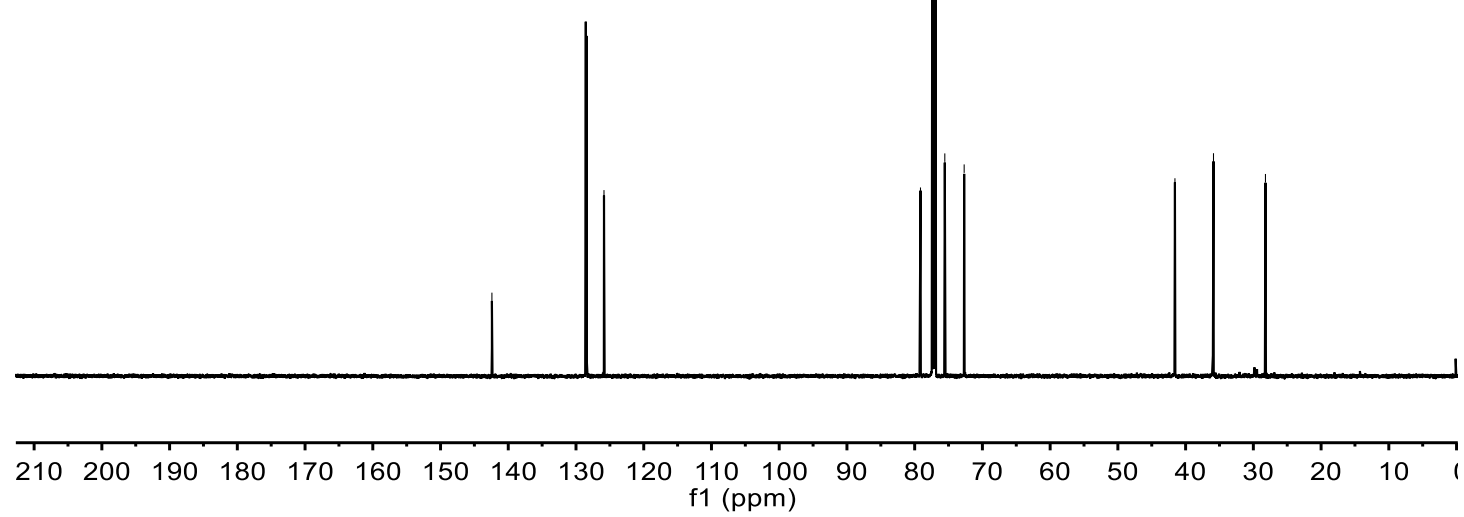

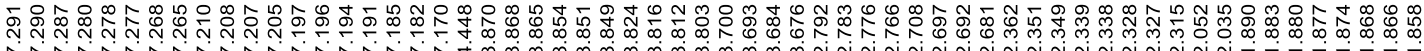

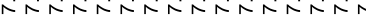

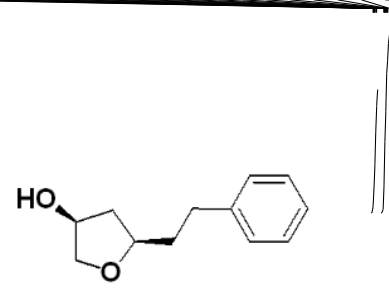

$(3 S, 5 R)-4 a w$

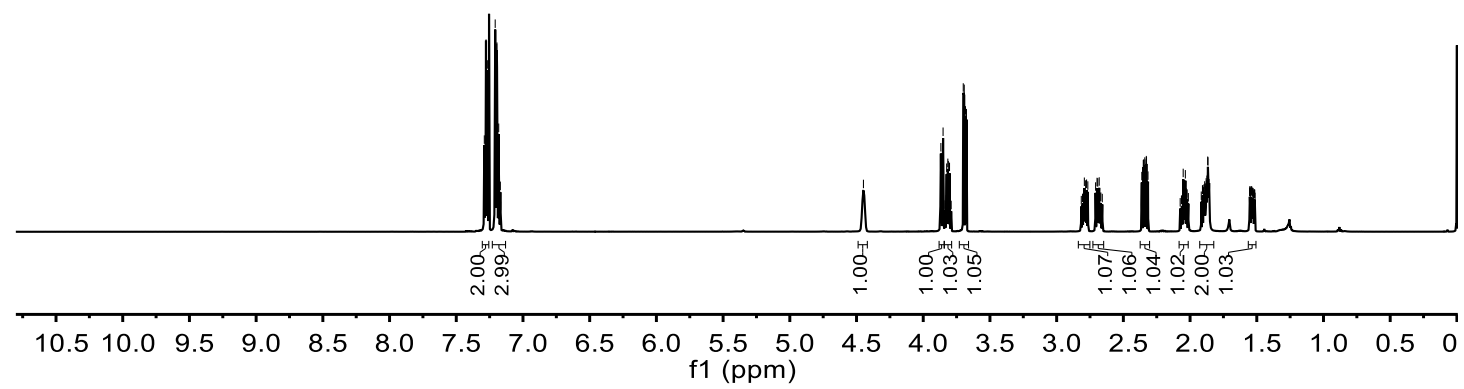




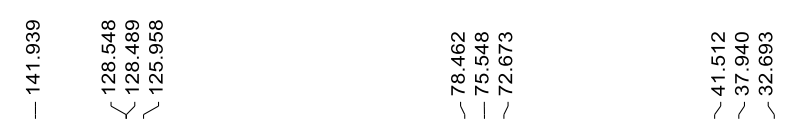

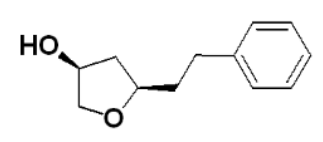

$(3 S, 5 R)-4 a w$
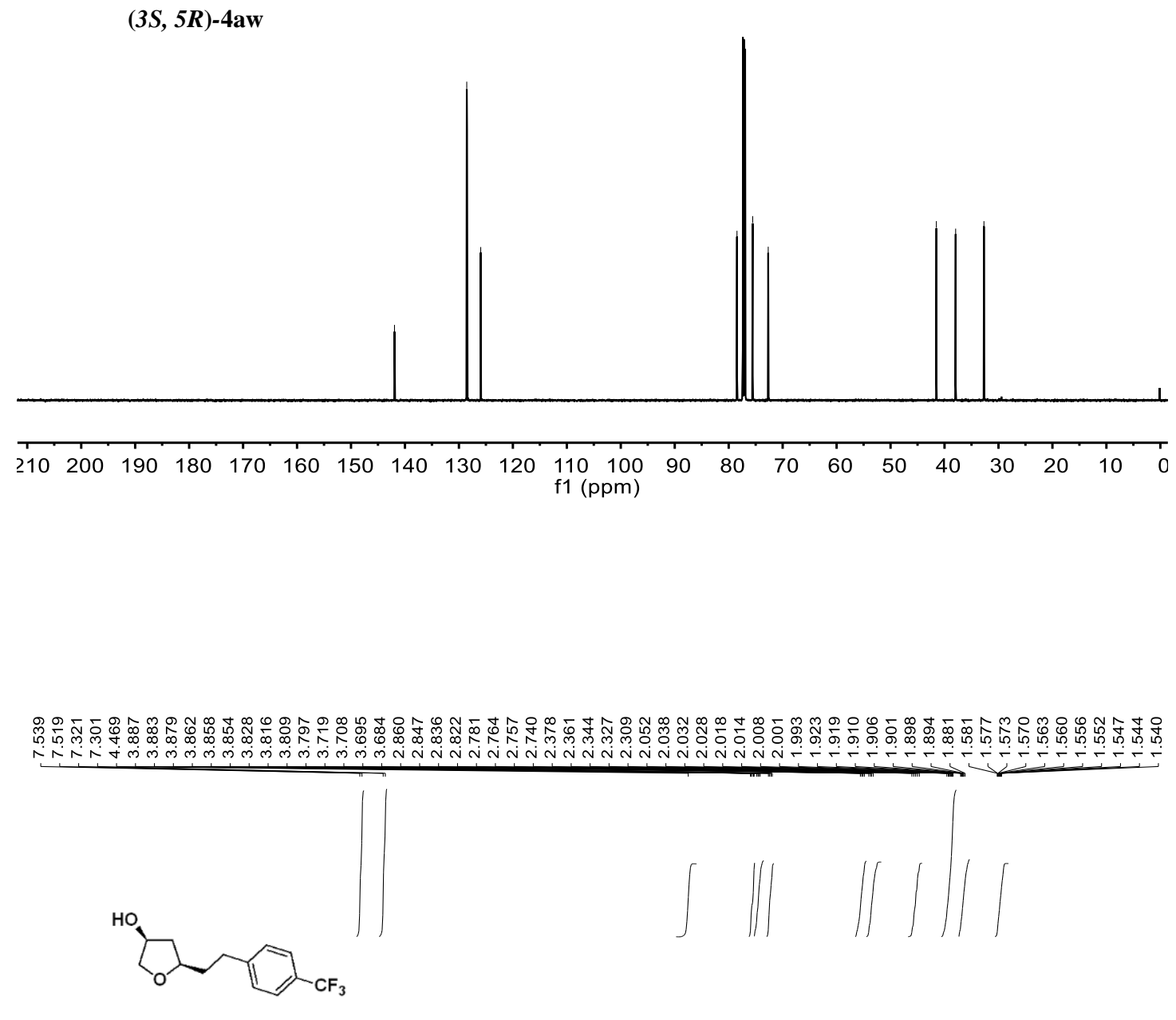

$(3 S, 5 R)-4 a x$

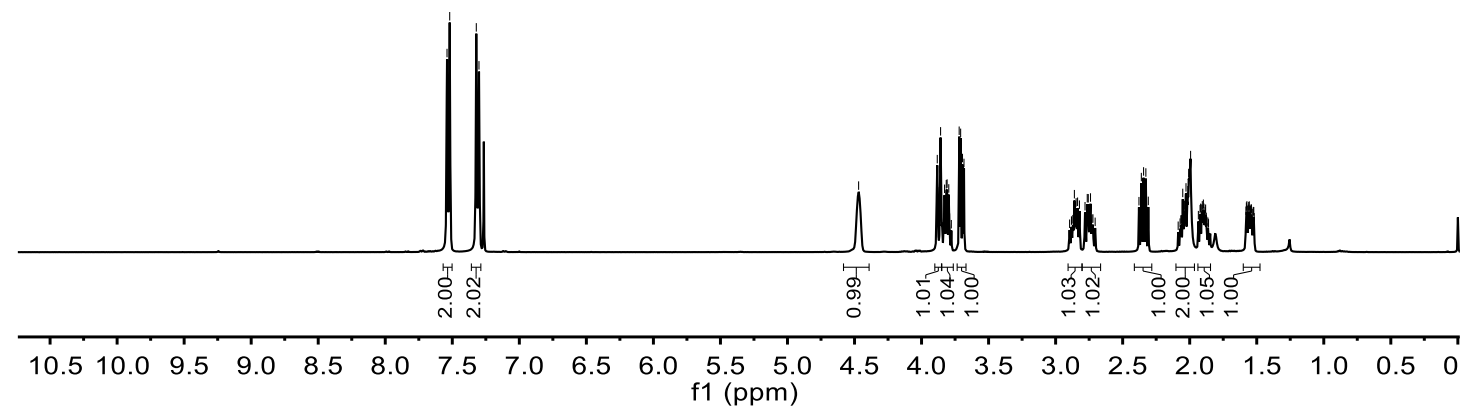


<smiles>OC1COC(CCc2ccc(C(F)(F)F)cc2)C1</smiles>

$(3 S, 5 R)-4 a x$

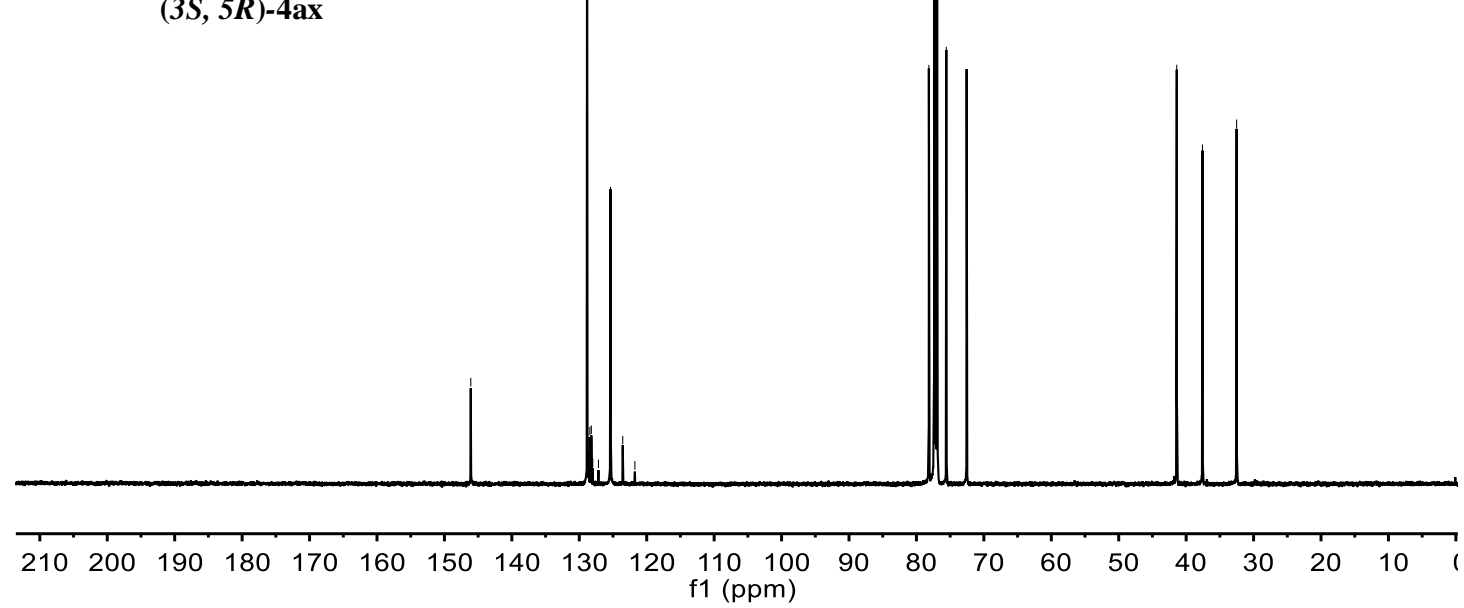

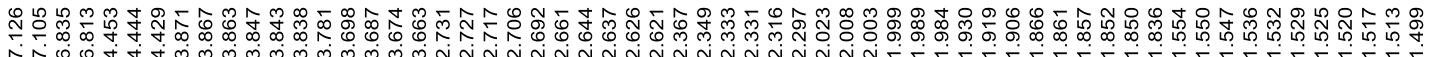
0
9<smiles>COc1ccc(CCC2CC(O)CO2)cc1</smiles>

$(3 S, 5 R)-4 a y$

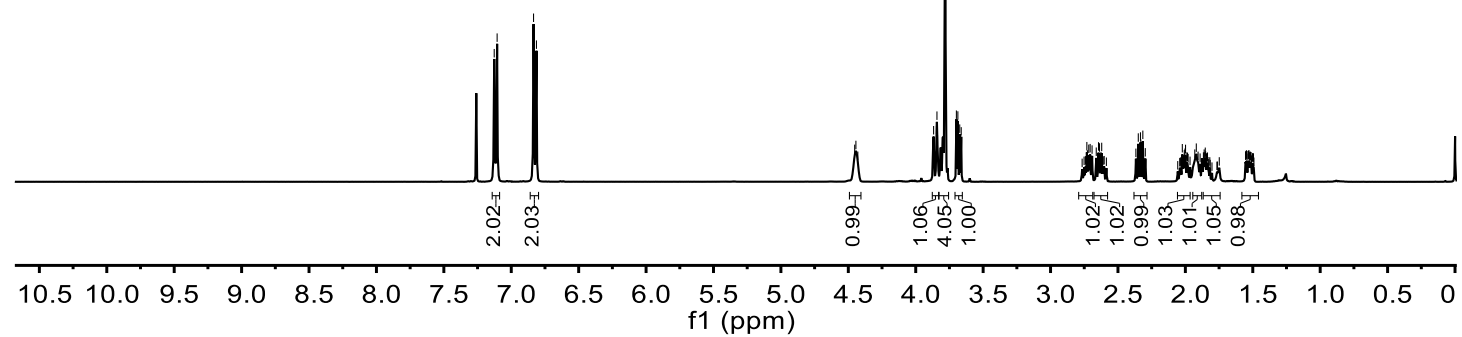




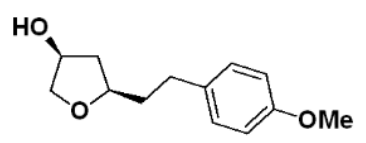

$(3 S, 5 R)-4 a y$

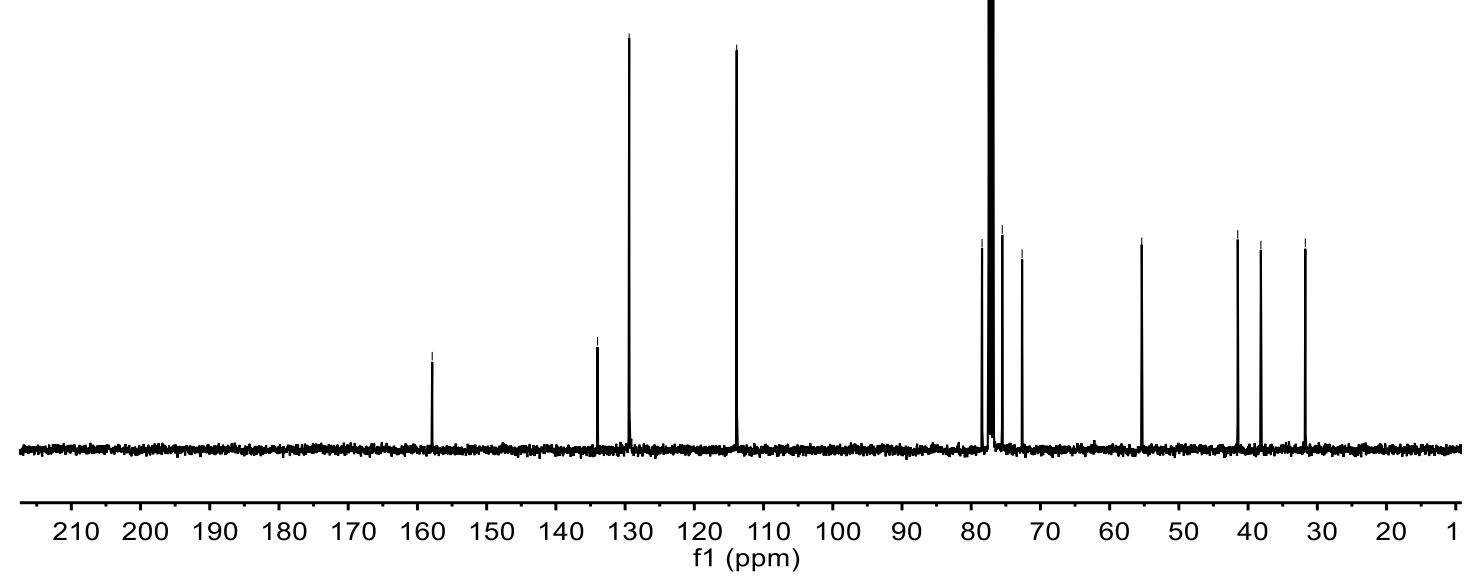

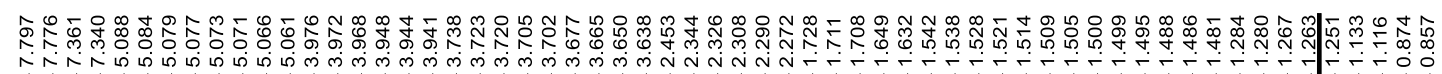

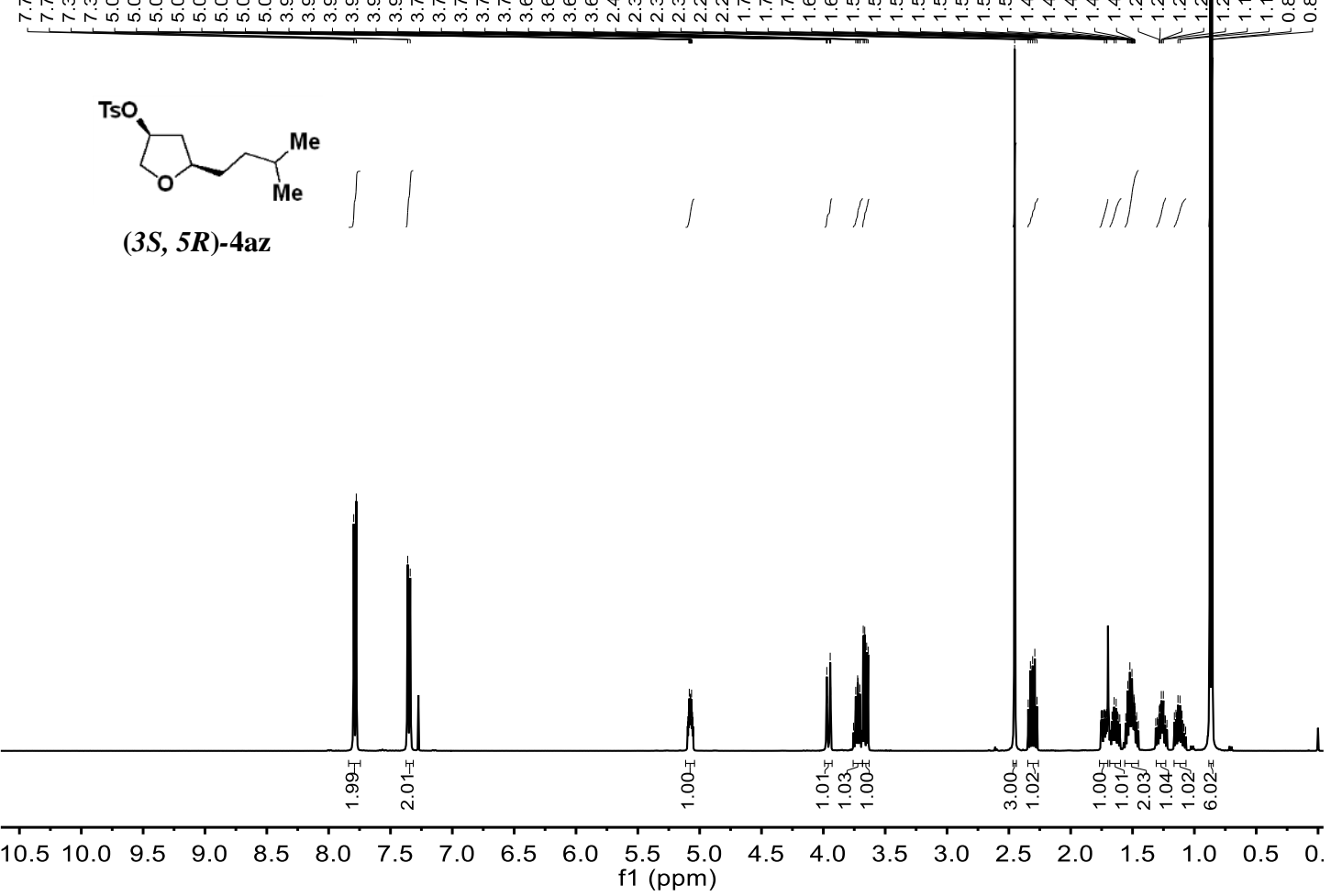




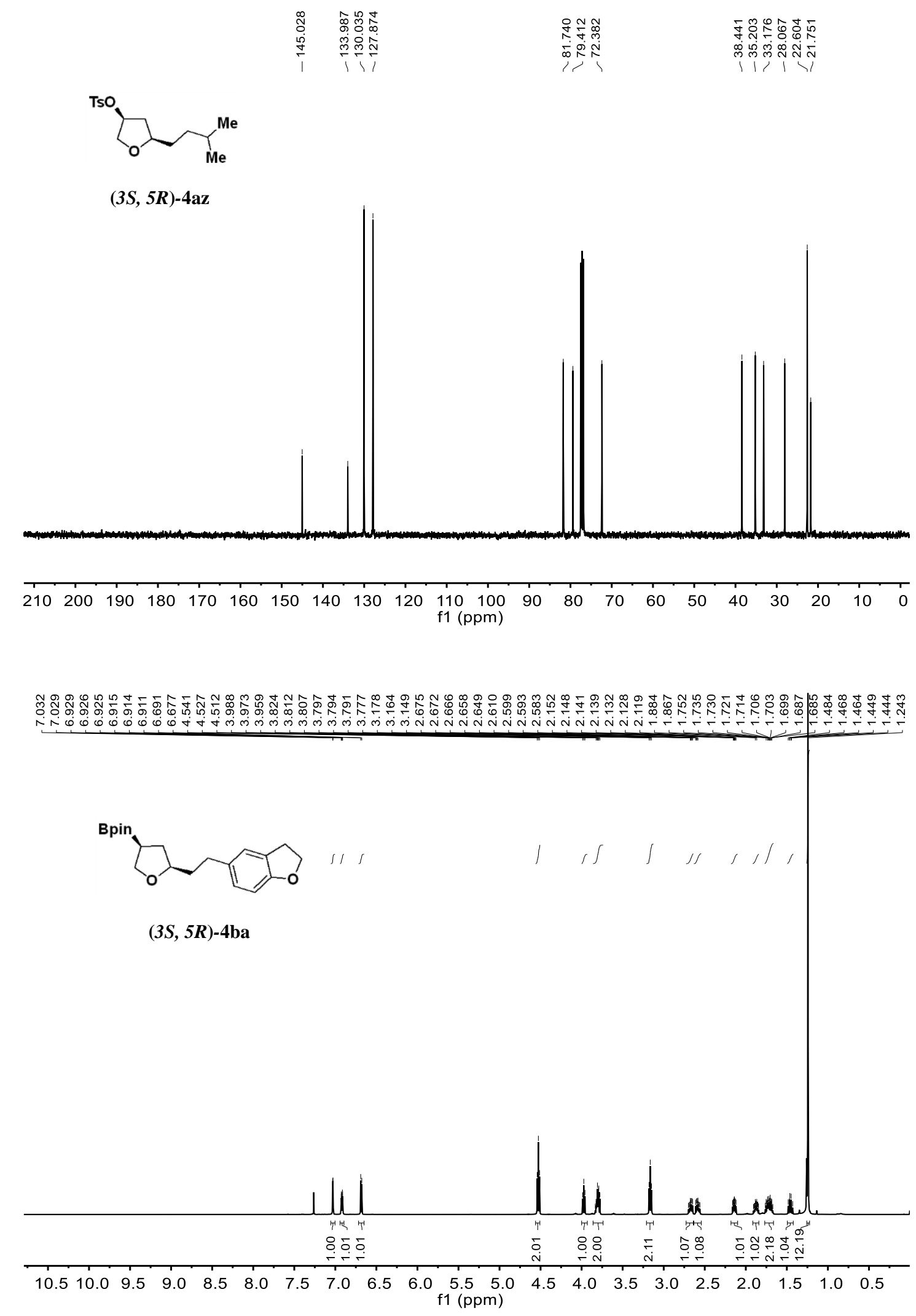


Bpin<smiles>CC1COC(CCc2ccc3c(c2)CCO3)C1</smiles>

$(3 S, 5 R)-4 \mathrm{ba}$

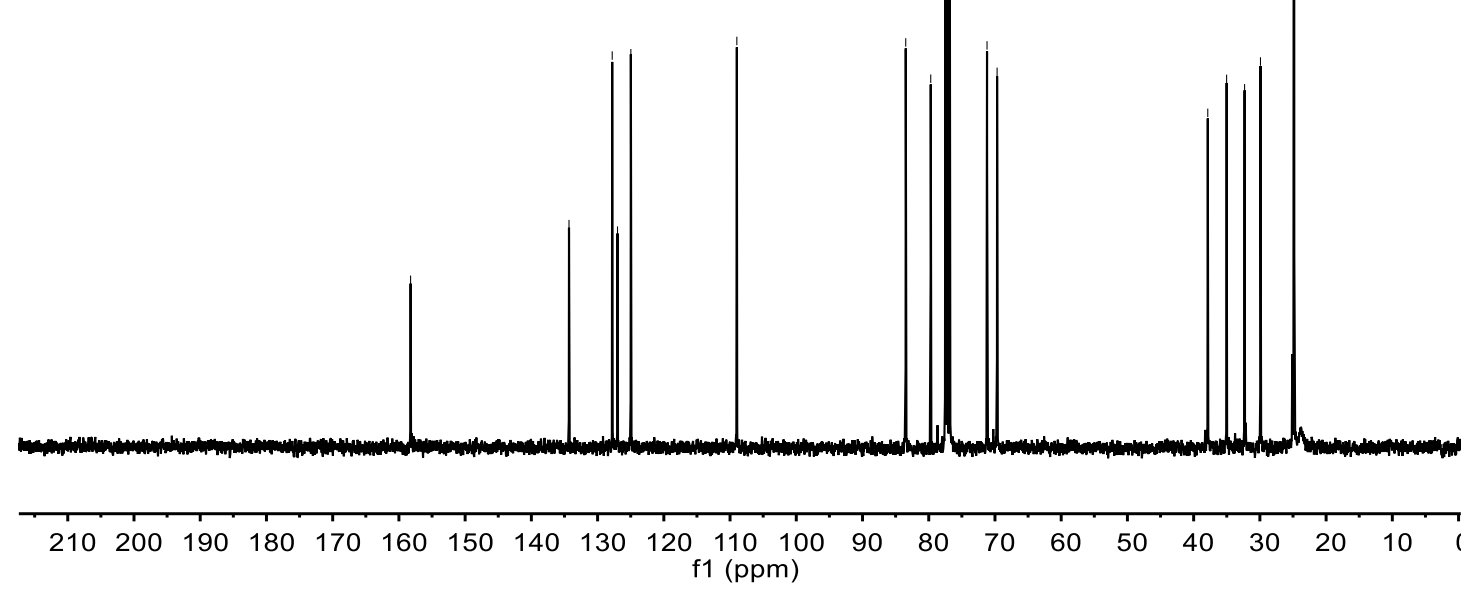

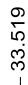<smiles>c1ccc(C[C@@H]2COC(CCc3ccc4c(c3)CCO4)C2)cc1</smiles>

$(3 S, 5 R)-4 b a$
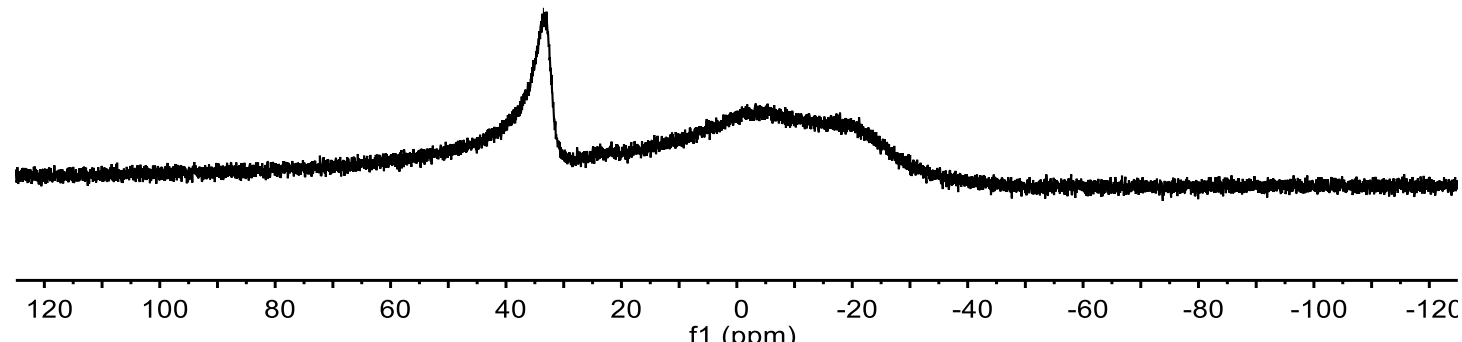

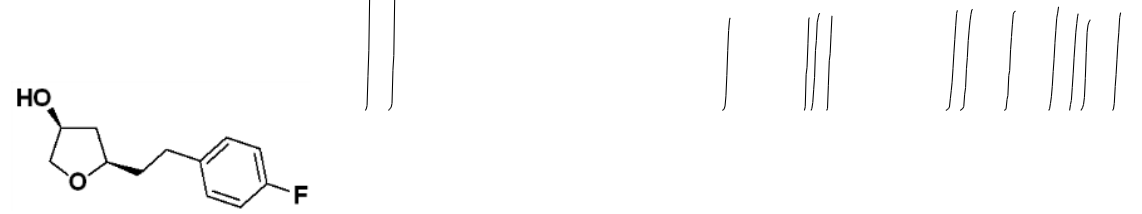

$(3 S, 5 R)-4 b b$

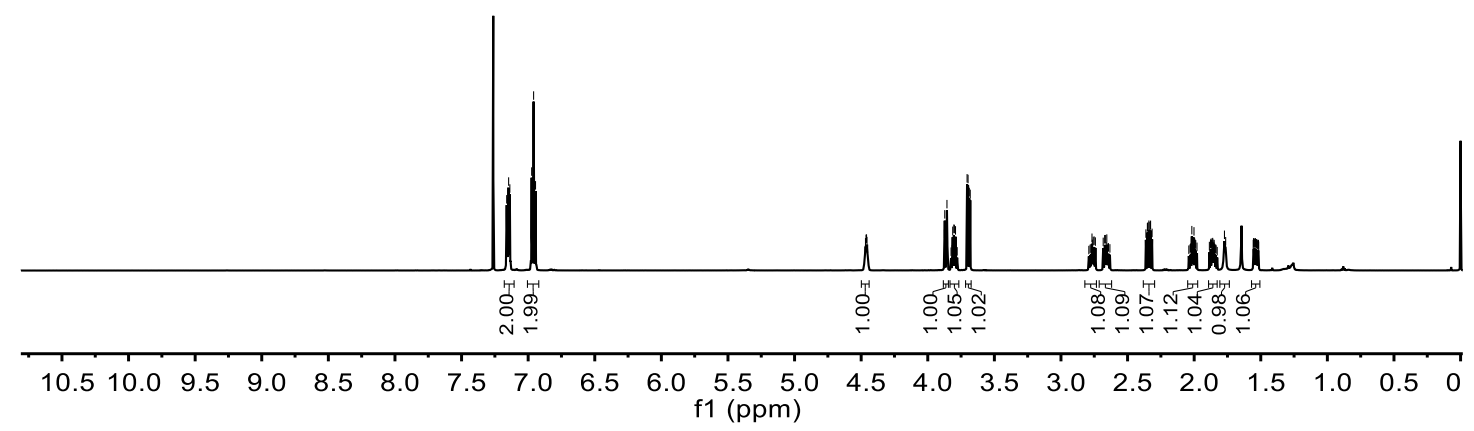

t.

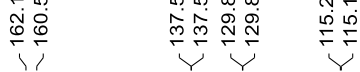

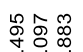

广我离

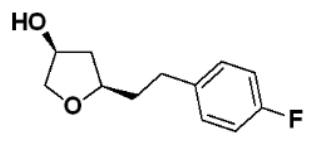

$(3 S, 5 R)-4 b b$

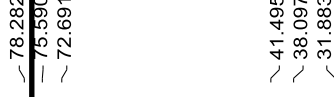

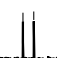

$\begin{array}{lllllllllllllllllllll}210 & 200 & 190 & 180 & 170 & 160 & 150 & 140 & 130 & 120 & 110 & 100 & 90 & 80 & 70 & 60 & 50 & 40 & 30 & 20 & 10\end{array}$ f1 $(\mathrm{ppm})$ 


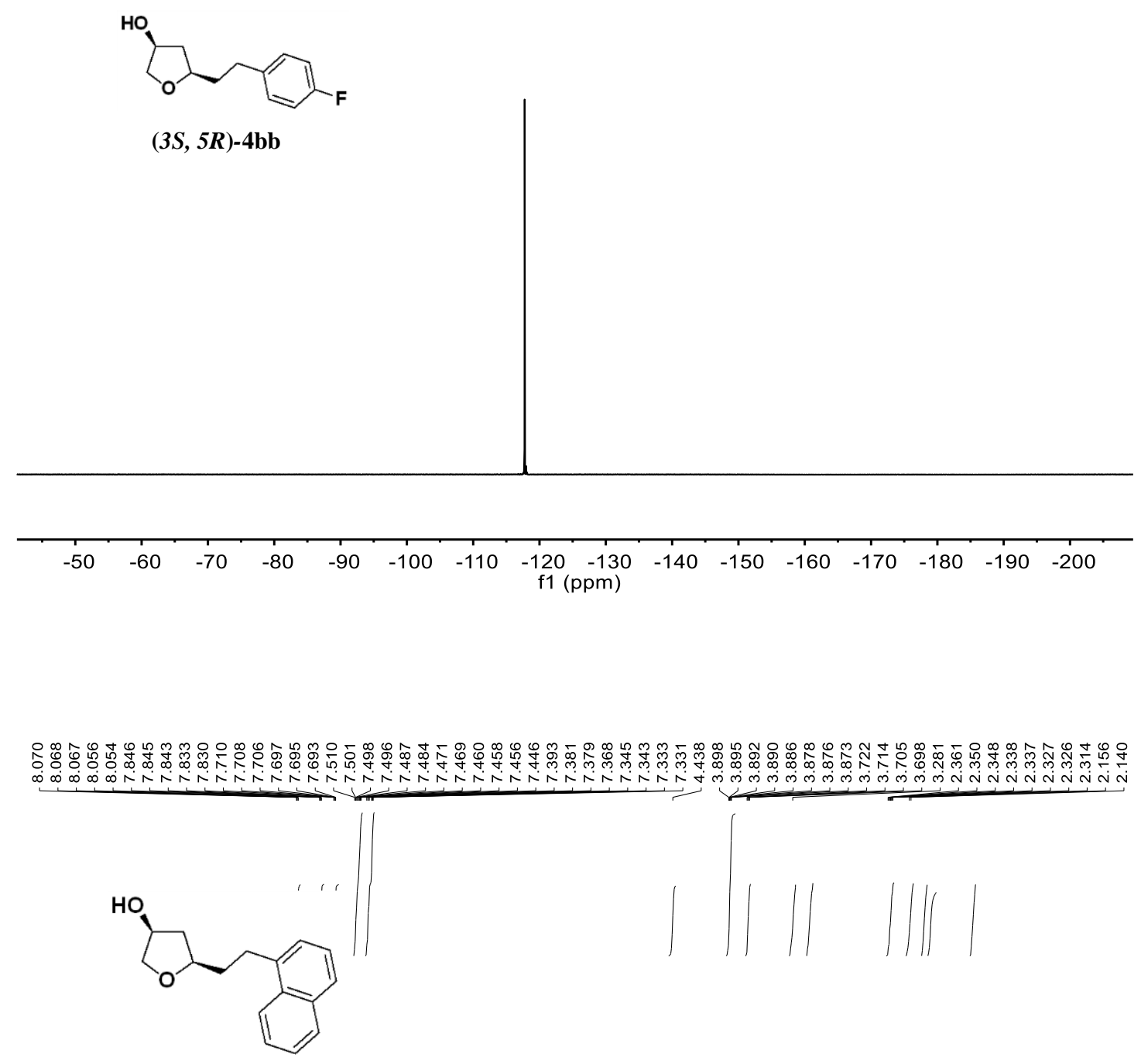

(3S, 5R)-4bc

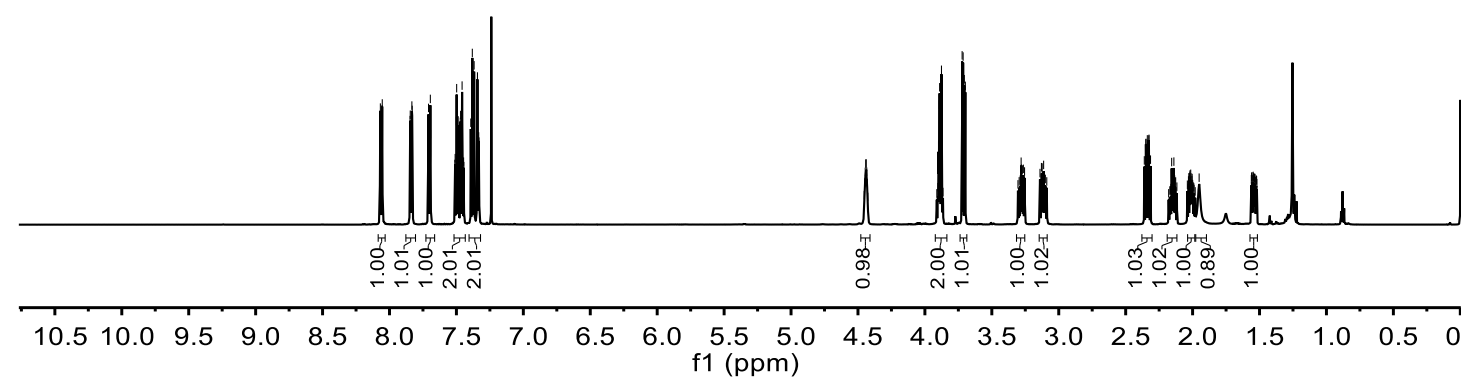




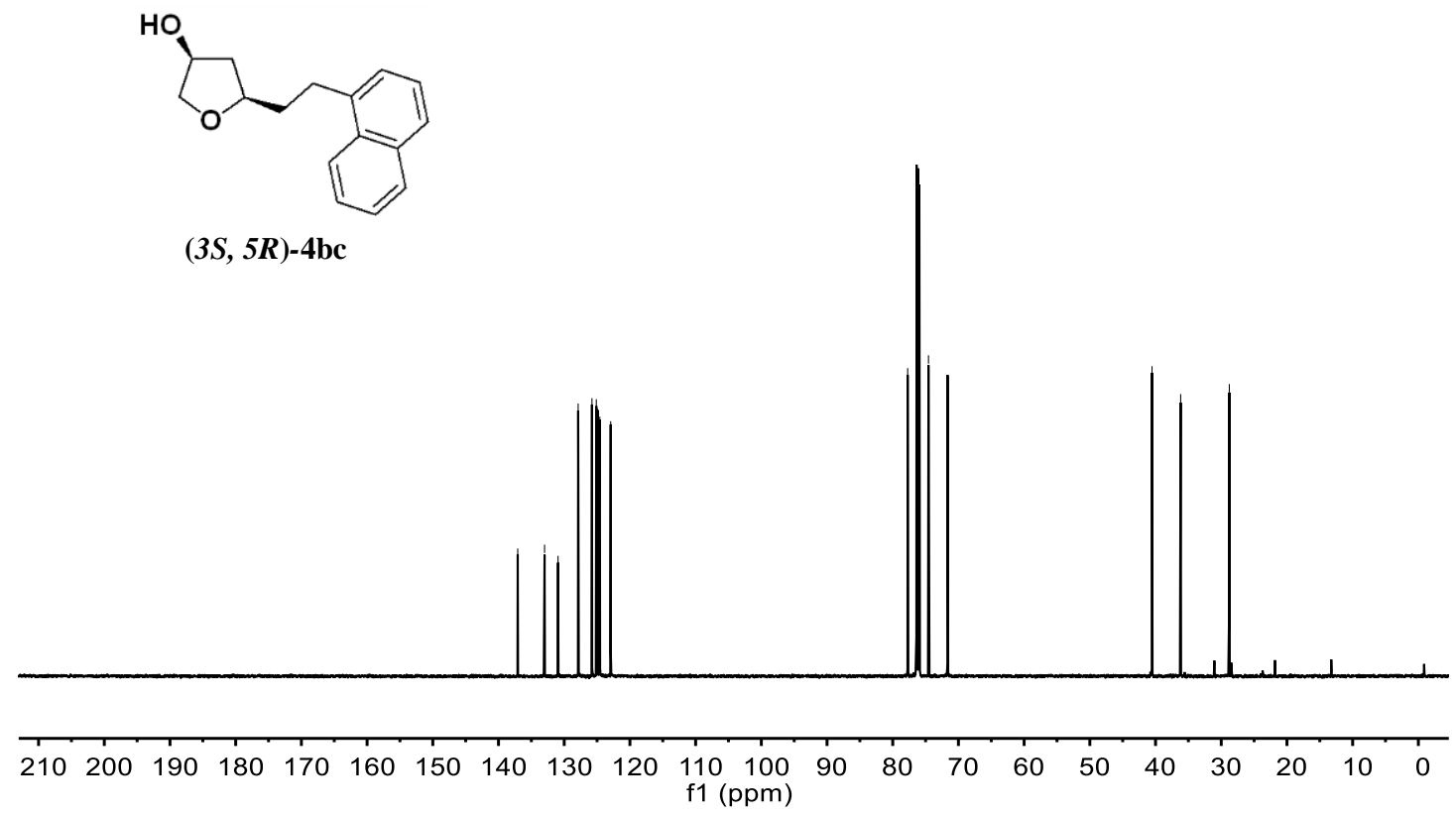

$(3 S, 5 R)-4 b c$

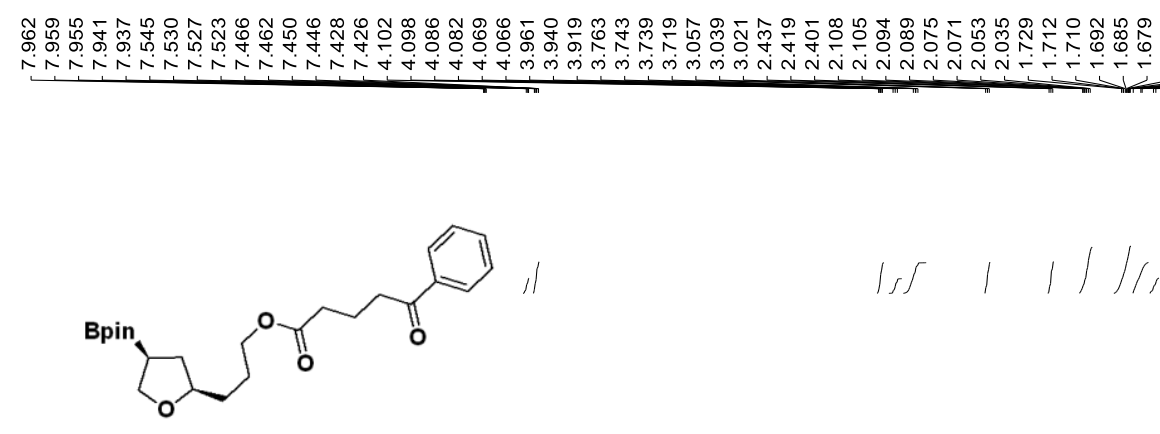

$(3 S, 5 R)-4 b d$

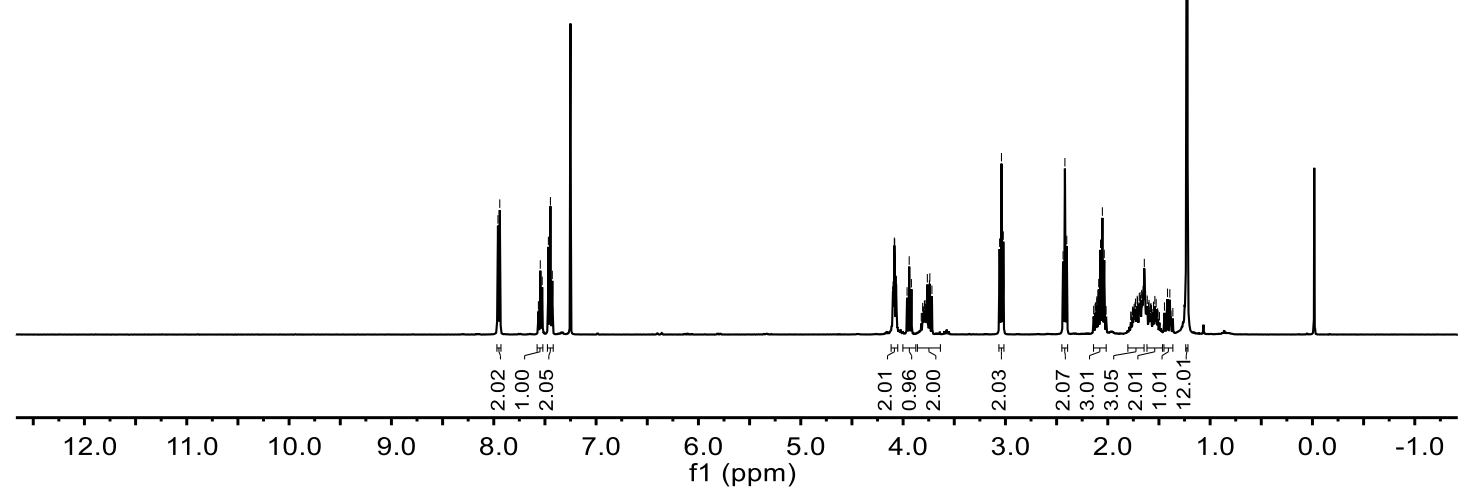




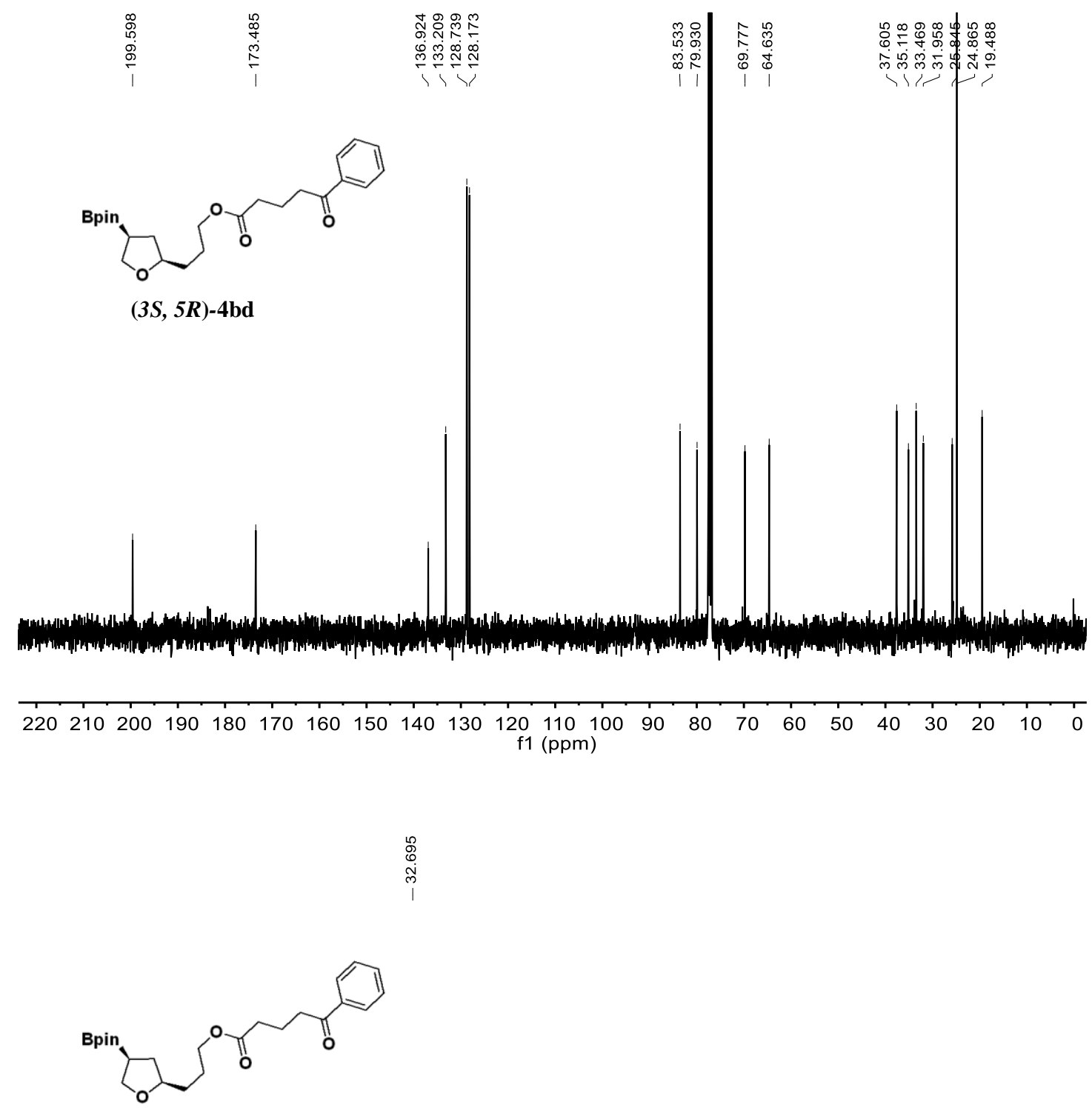

(3S, 5R)-4bd

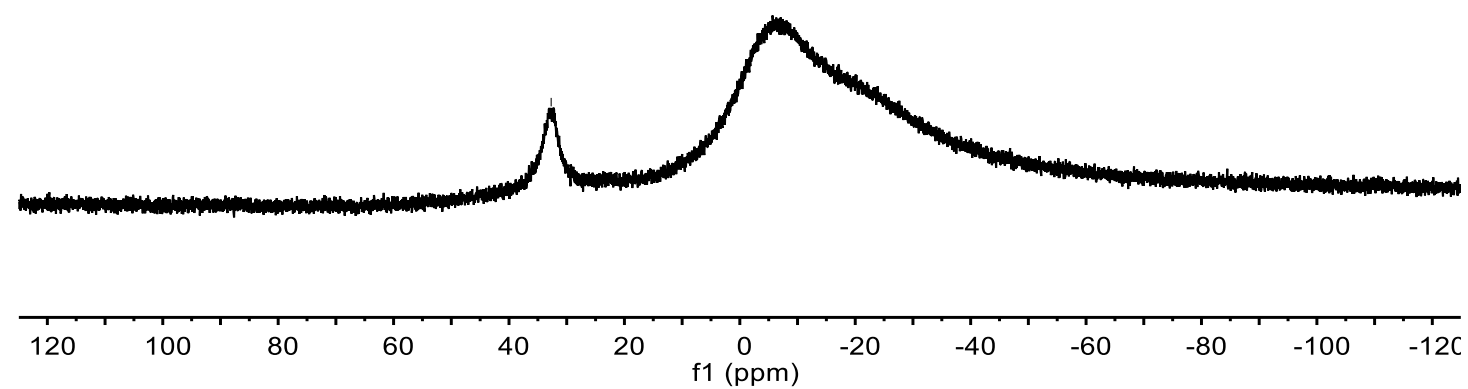


<smiles>Cc1cccc(C)c1OCCCC1CC(Cc2ccccc2)CO1</smiles>

$(3 S, 5 R)-4 b e$
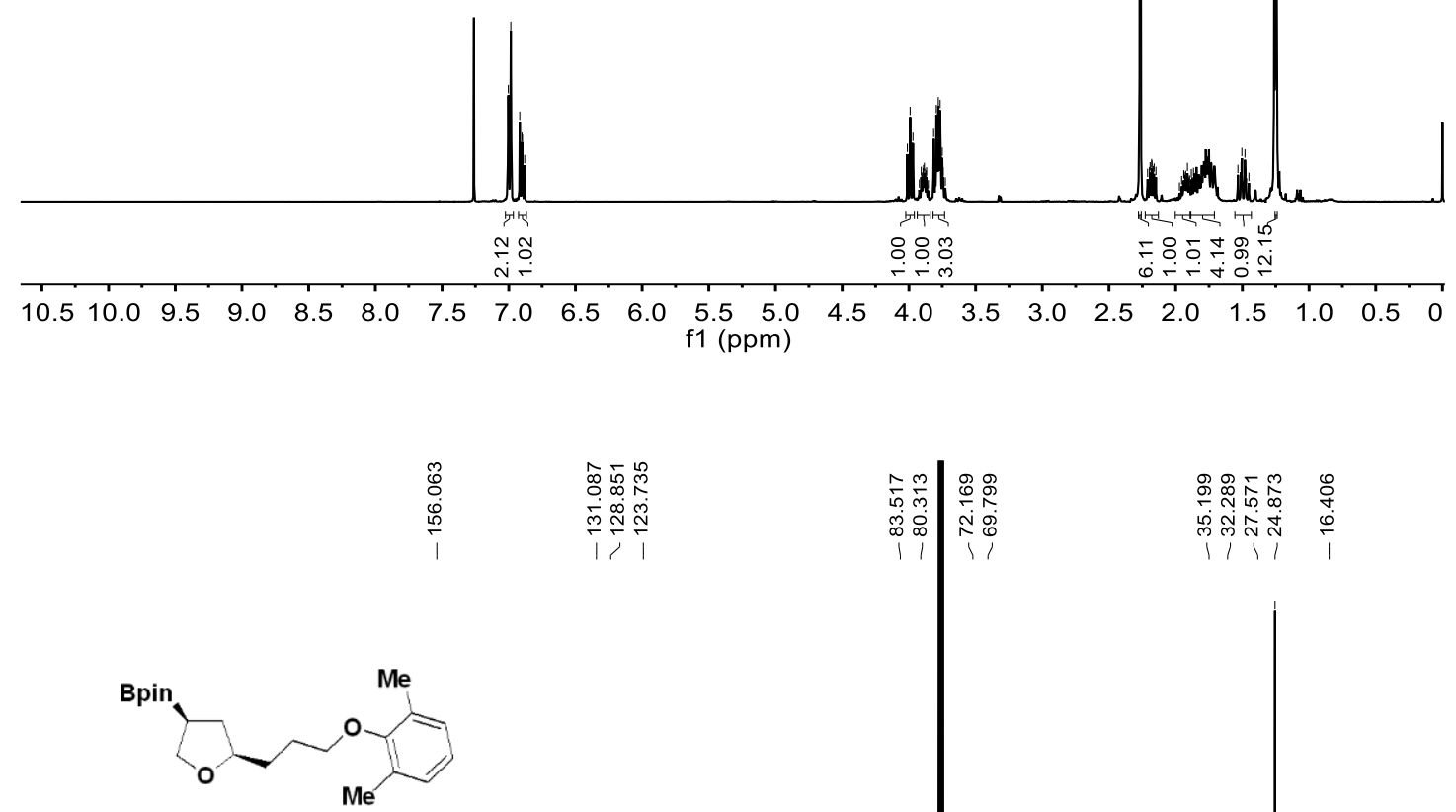

$(3 S, 5 R)-4 b e$

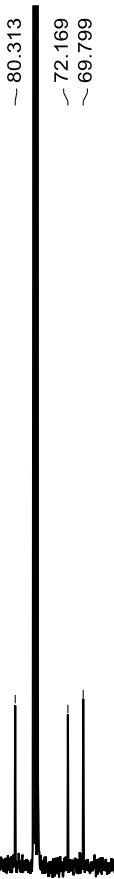

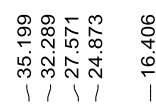

$20 \quad 210200190180 \quad 170160150 \quad 140 \quad 130 \quad 120 \quad 110 \quad 100 \quad 90$

f1 (ppm) 


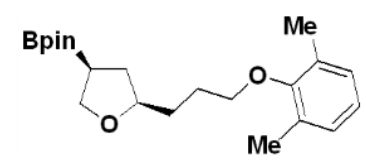

$(3 S, 5 R)-4$ be

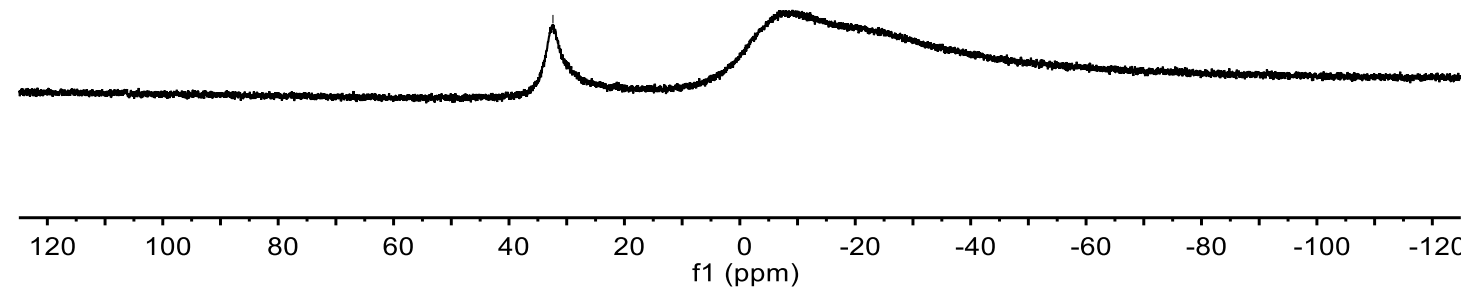

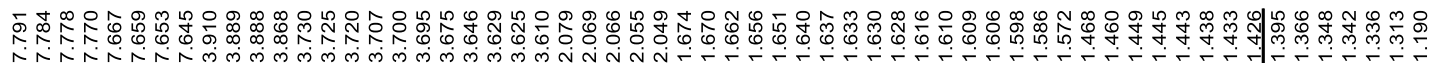

Nivistion

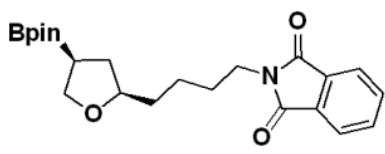

, 11

,$\quad 1 / 1$

$(3 S, 5 R)-4 b f$

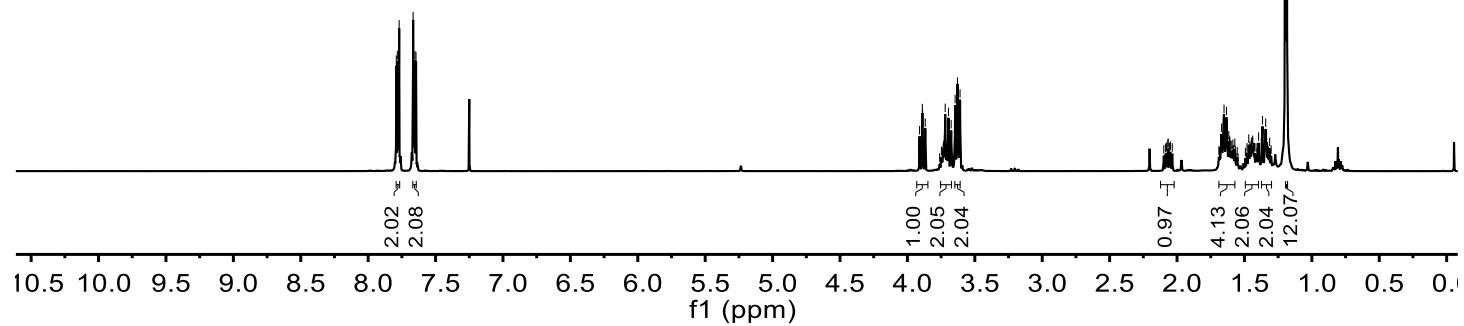




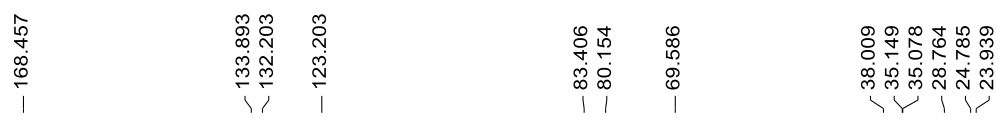<smiles>O=C1c2ccccc2C(=O)N1CCCCC1CC(Cc2ccccc2)CO1</smiles>

(3S, 5R)-4bf

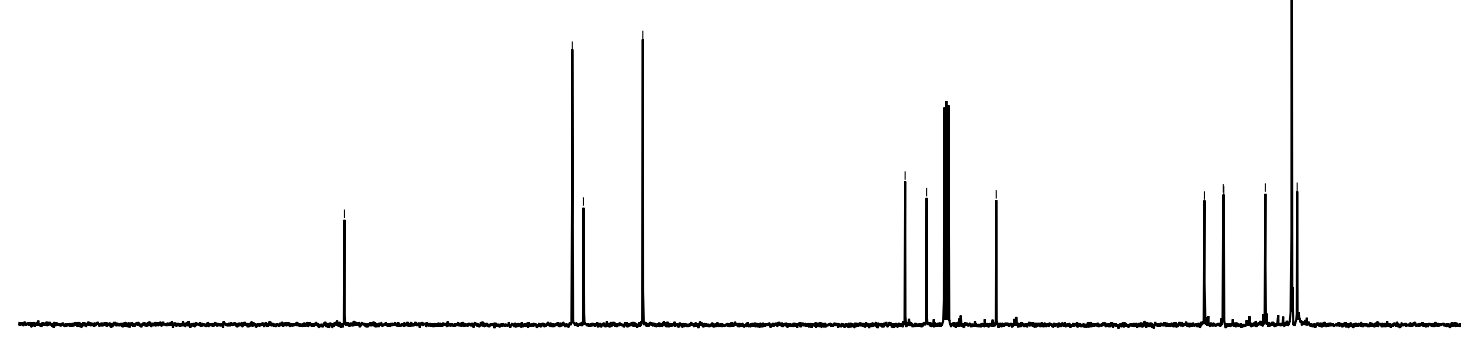

$\begin{array}{llllllllllllllllllllll}210 & 200 & 190 & 180 & 170 & 160 & 150 & 140 & 130 & 120 & 110 & 100 & 90 & 80 & 70 & 60 & 50 & 40 & 30 & 20 & 10 & c\end{array}$ $\mathrm{f} 1(\mathrm{ppm})$

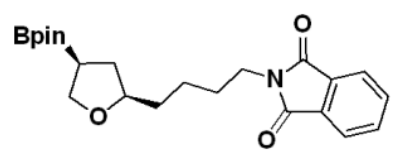

$(3 S, 5 R)-4 b f$
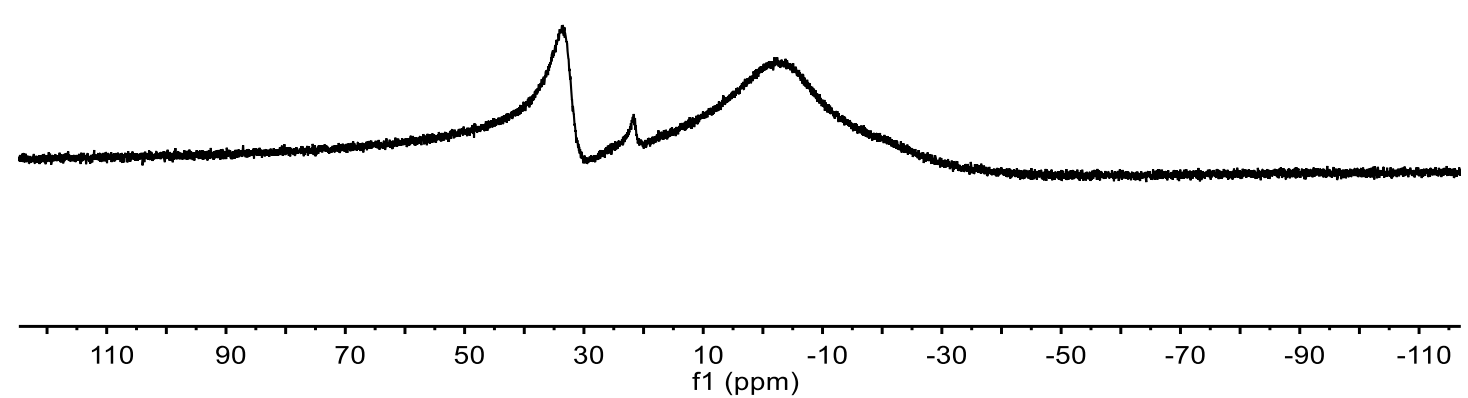
<smiles>[B](c1ccccc1)C1CCOC(CCCc2ccccc2)C1</smiles>

$(2 R, 4 R)-4 j$
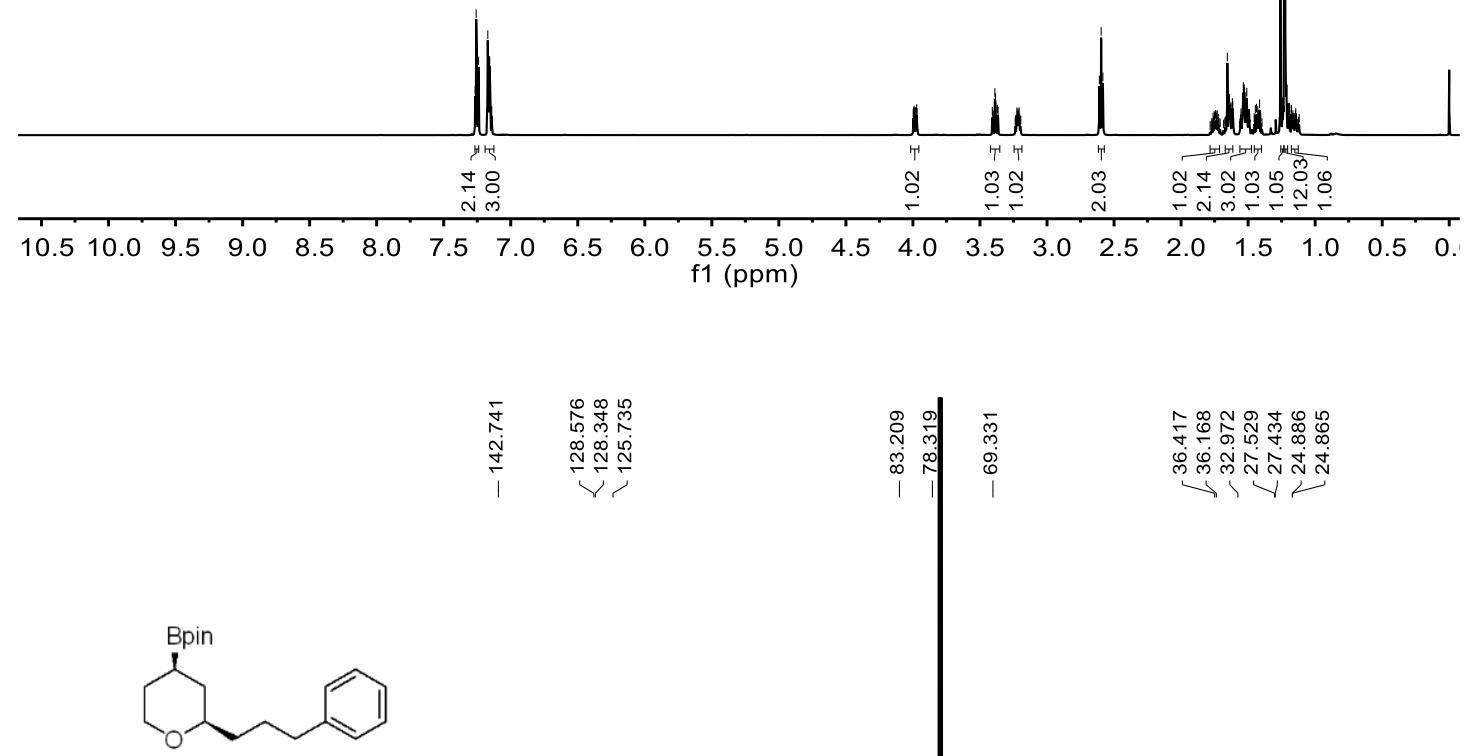

$(2 R, 4 R)-4 \mathrm{j}$

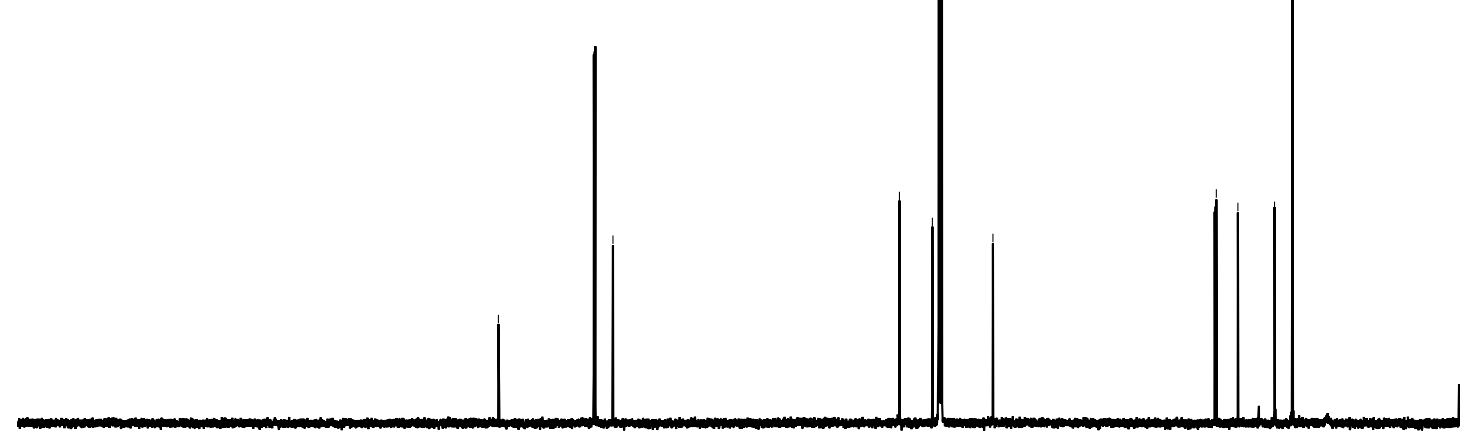

$\begin{array}{lllllllllllllllllllll}210 & 200 & 190 & 180 & 170 & 160 & 150 & 140 & 130 & 120 & 110 & 100 & 90 & 80 & 70 & 60 & 50 & 40 & 30 & 20 & 10\end{array}$ f1 (ppm) 


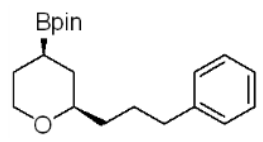

$(2 R, 4 R)-4 j$
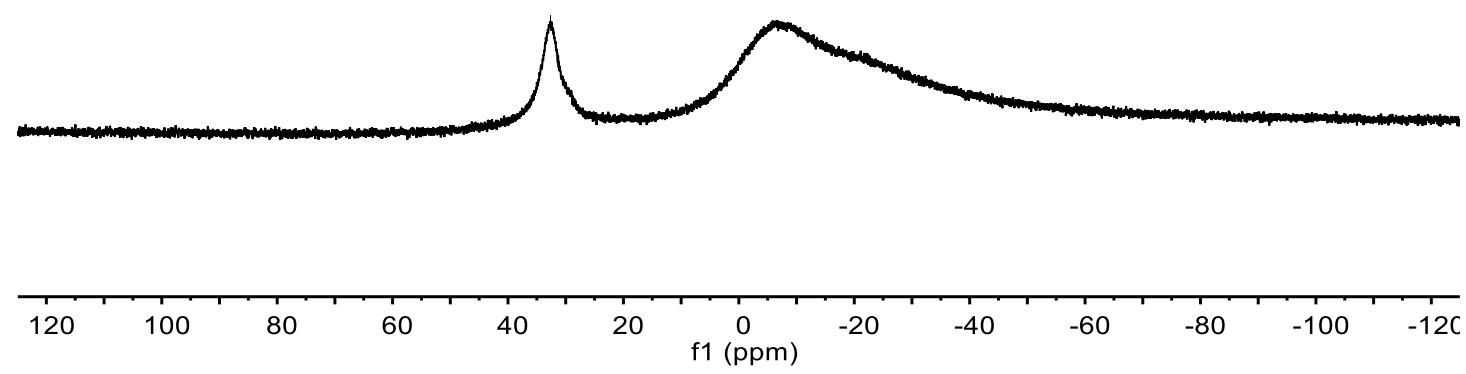

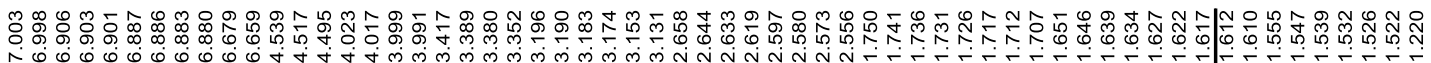

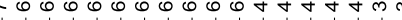<smiles>c1ccc([18Sc]C2CCOC(CCc3ccc4c(c3)CCO4)C2)cc1</smiles>

$(2 R, 4 R)-4 b g$

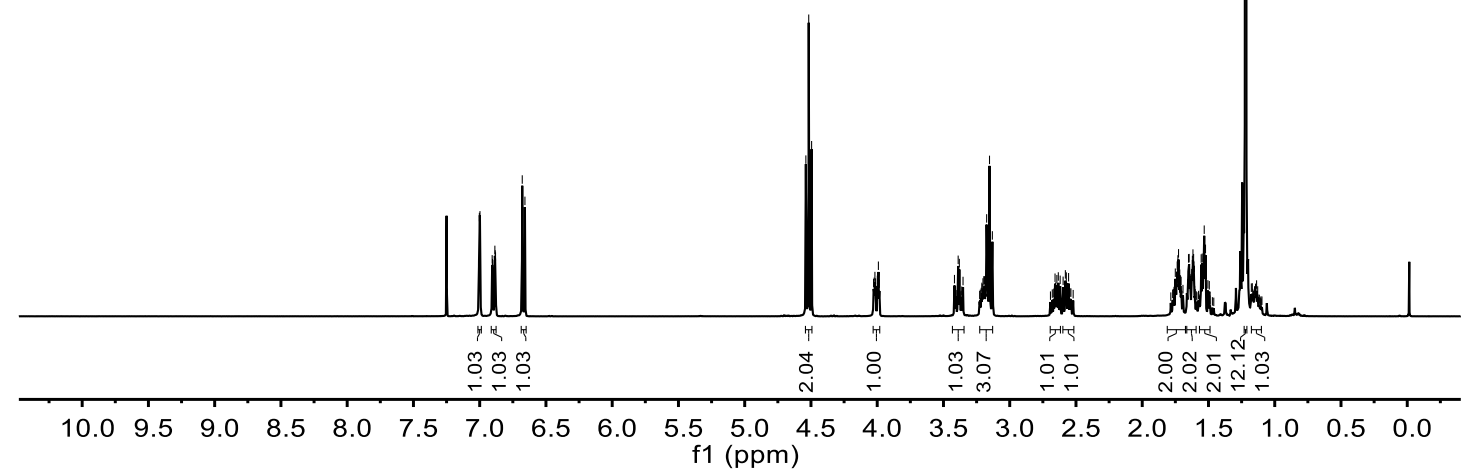




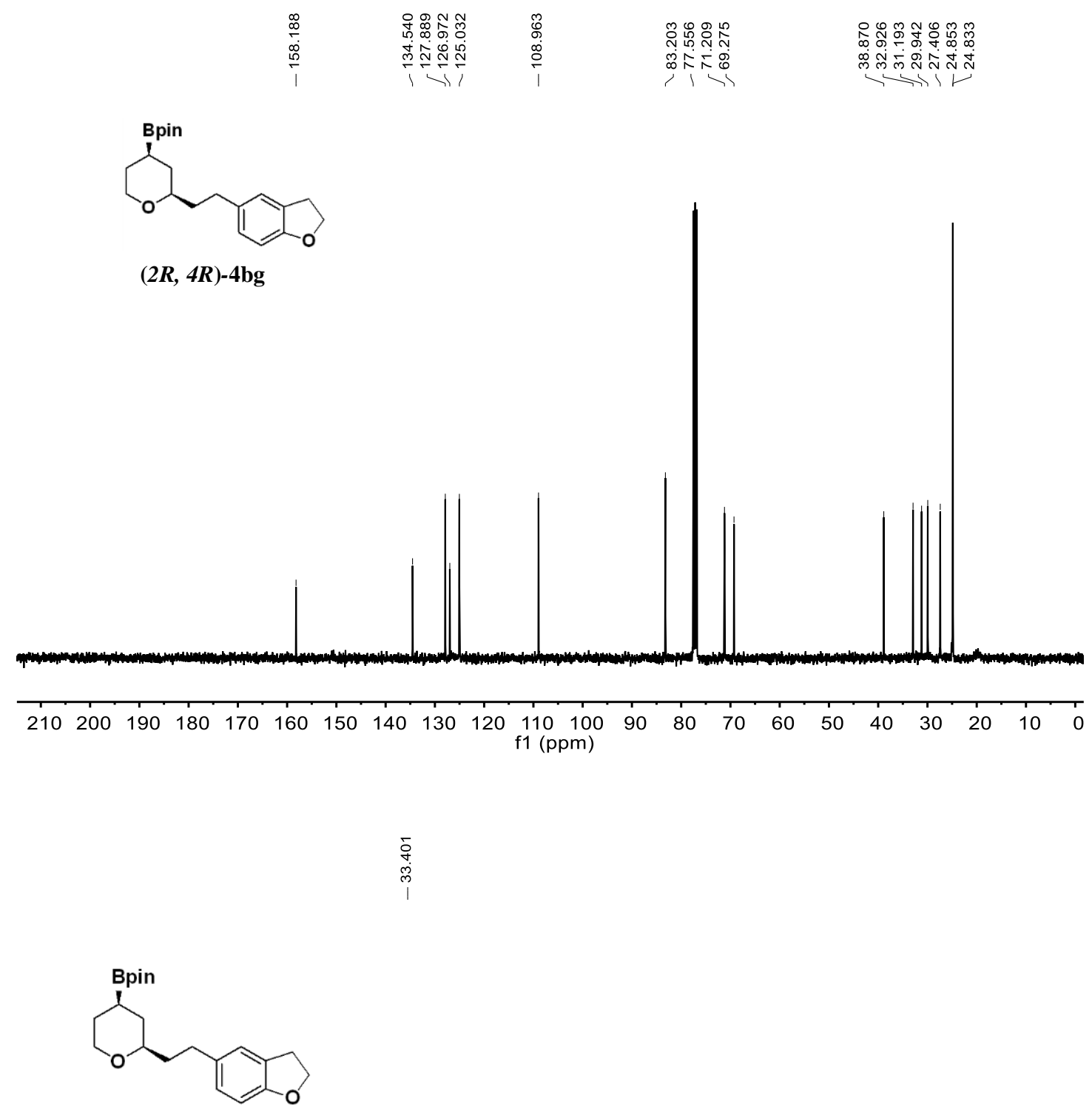

(2R, 4R)-4bg

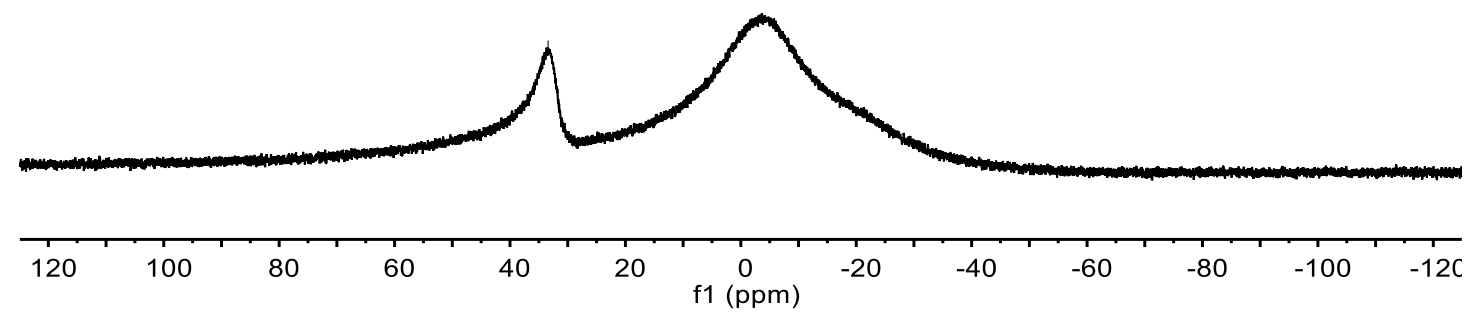




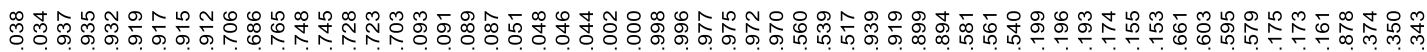

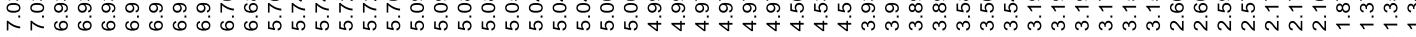<smiles>C=CC1COC(CCc2ccc3c(c2)CCO3)C1</smiles>

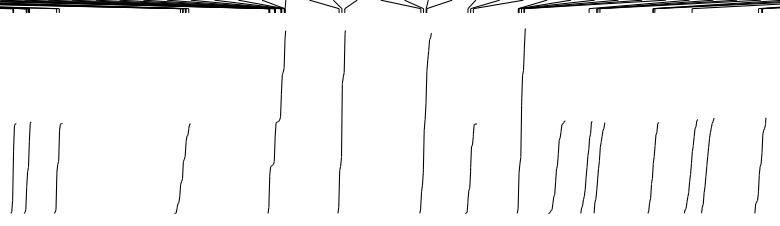

$(2 R, 4 R)-5$
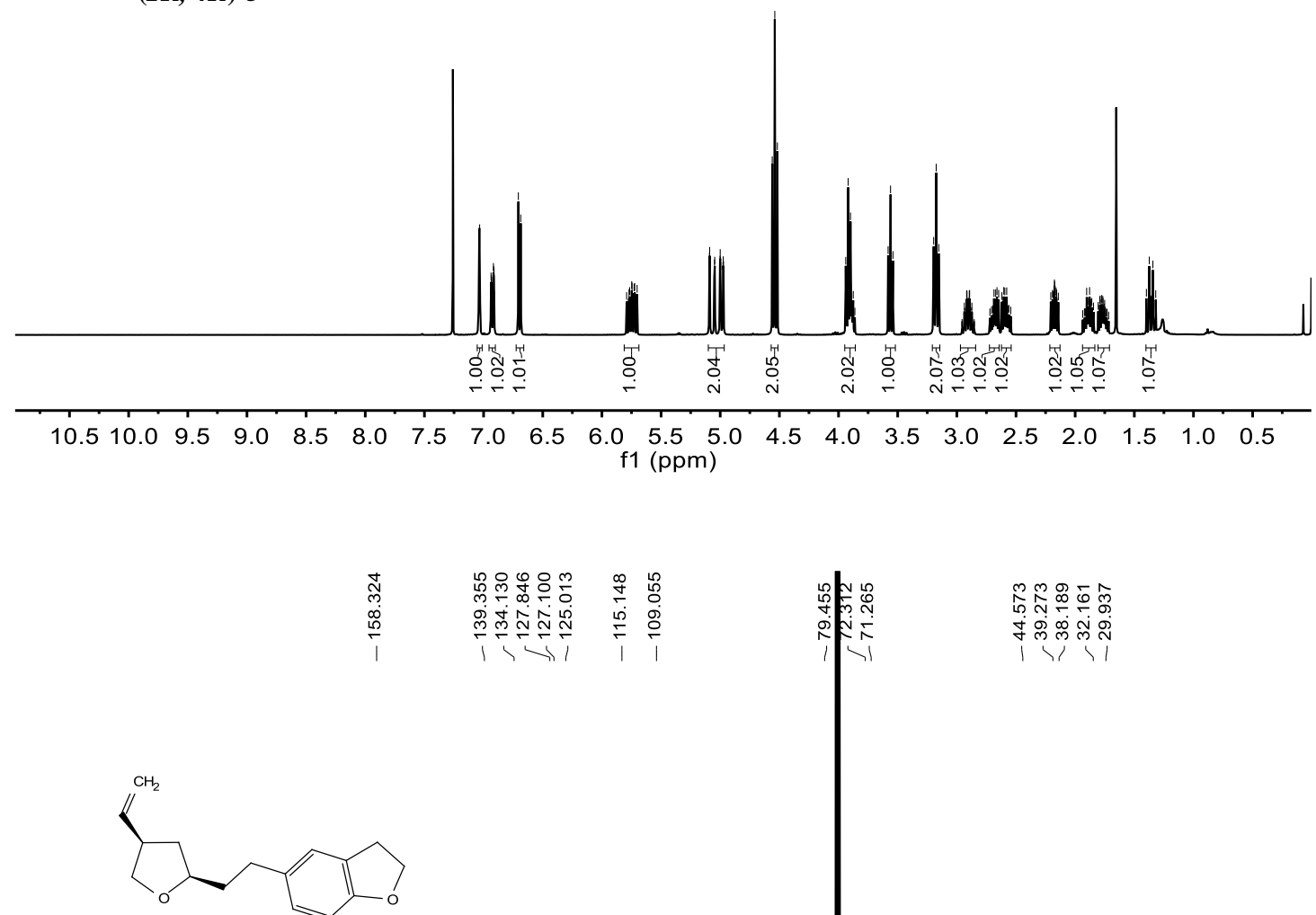

$(2 R, 4 R)-5$

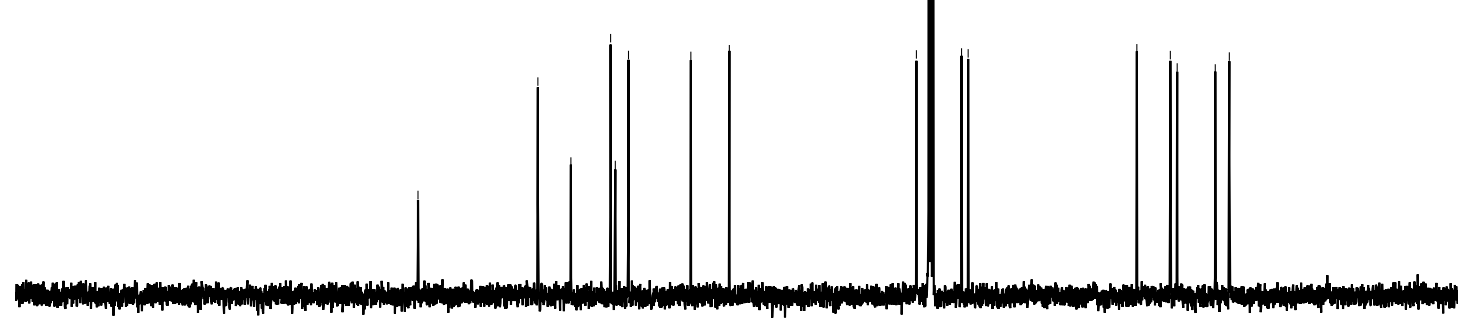

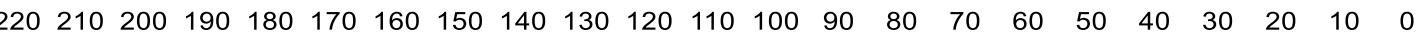
f1 (ppm) 

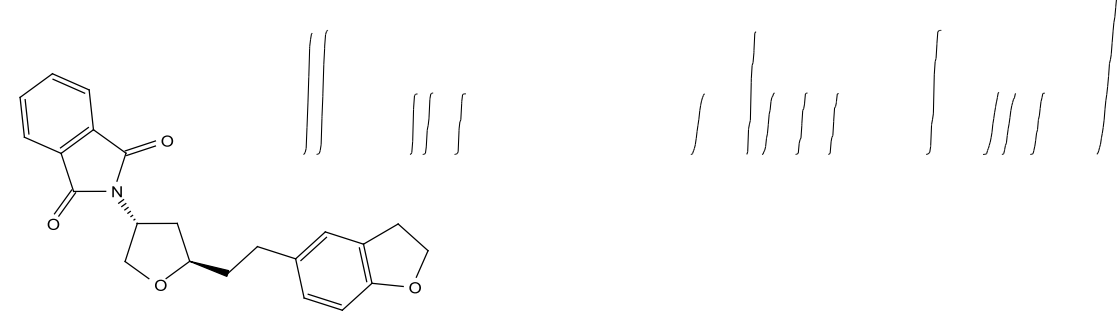

$(3 R, 5 R)-6$
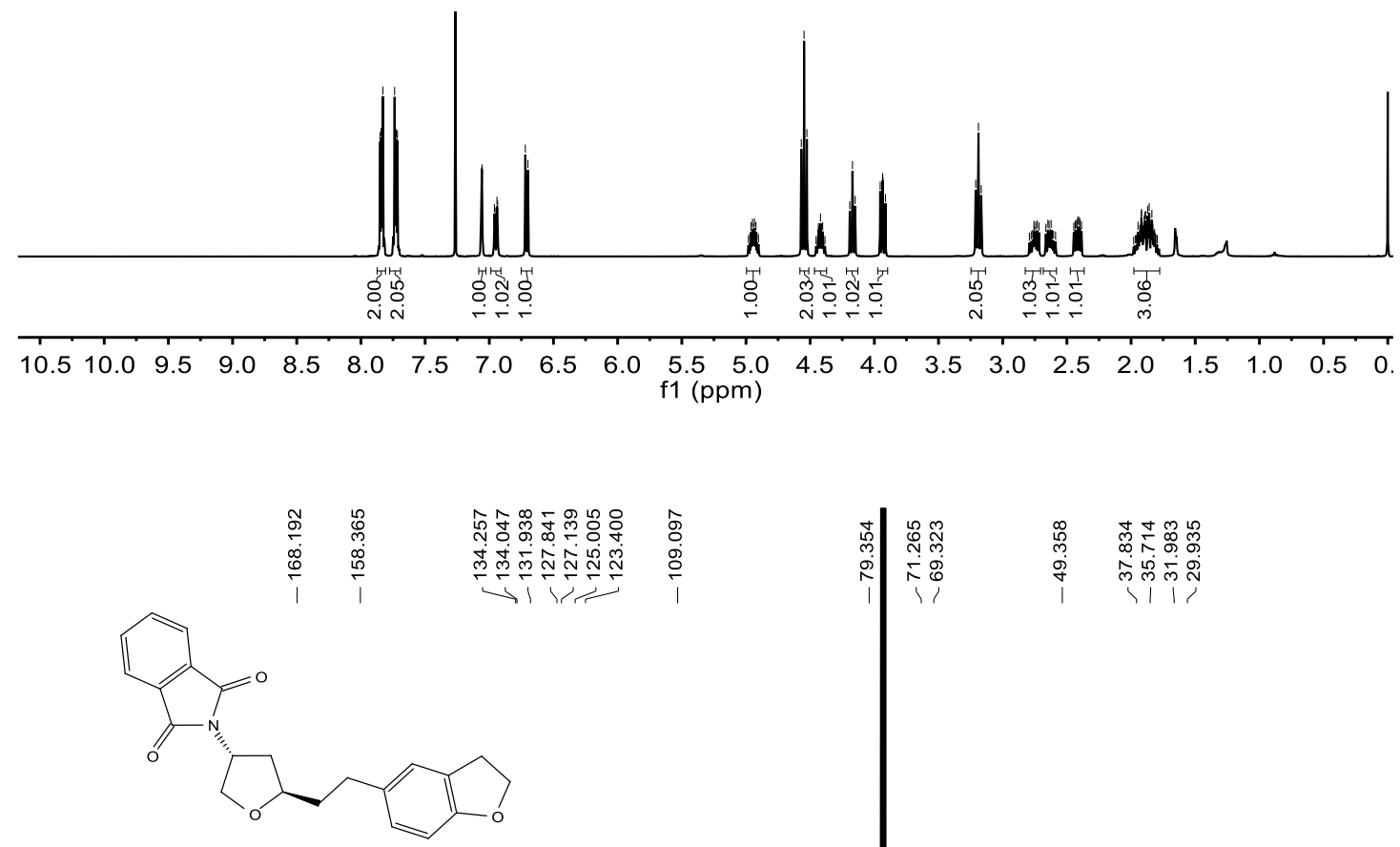

$(3 R, 5 R)-6$

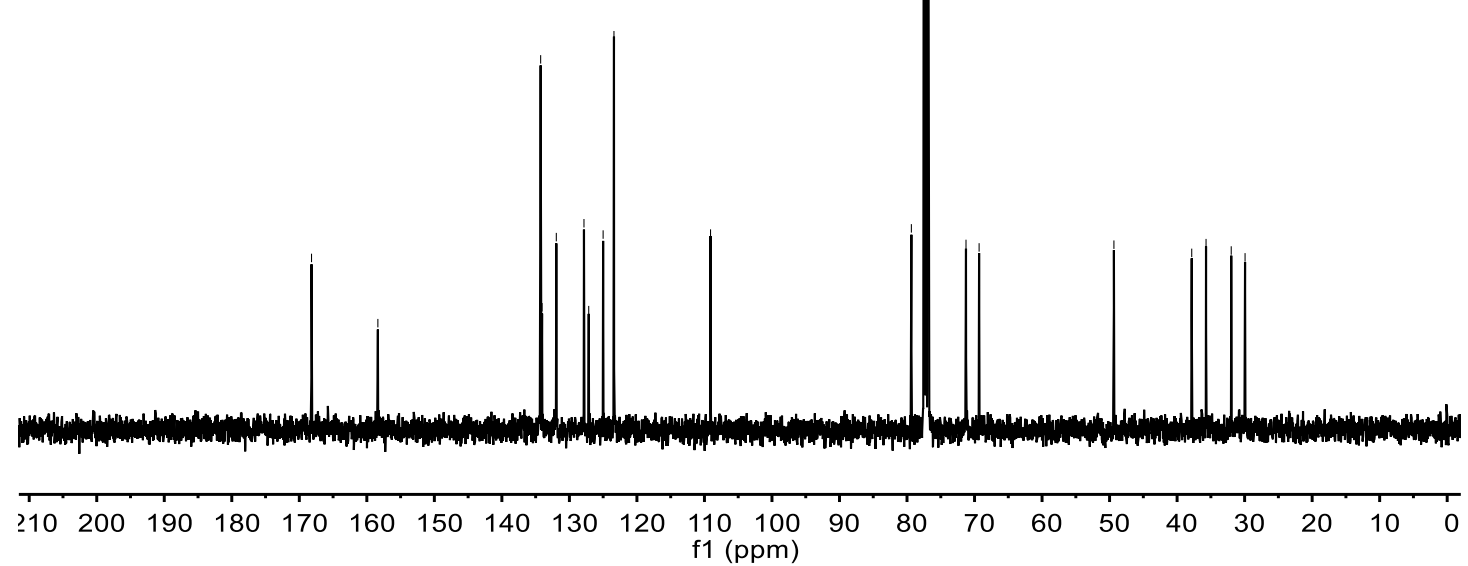




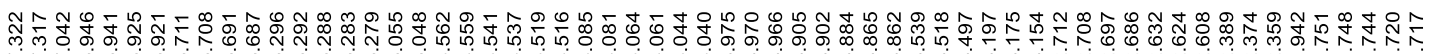

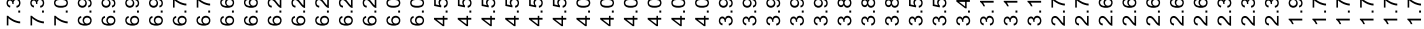
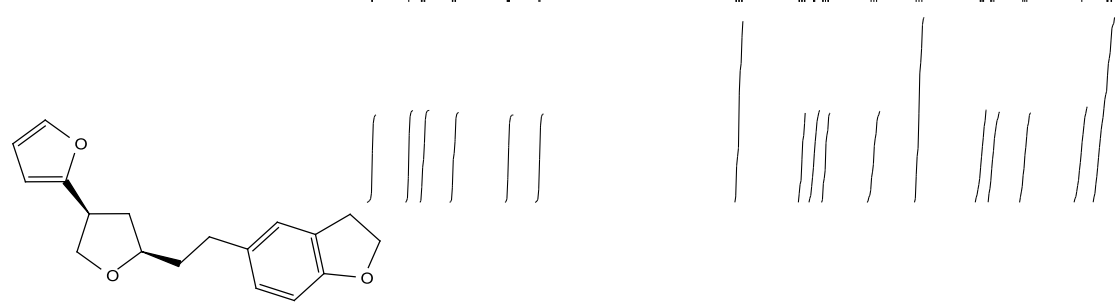

$(2 R, 4 S)-7$
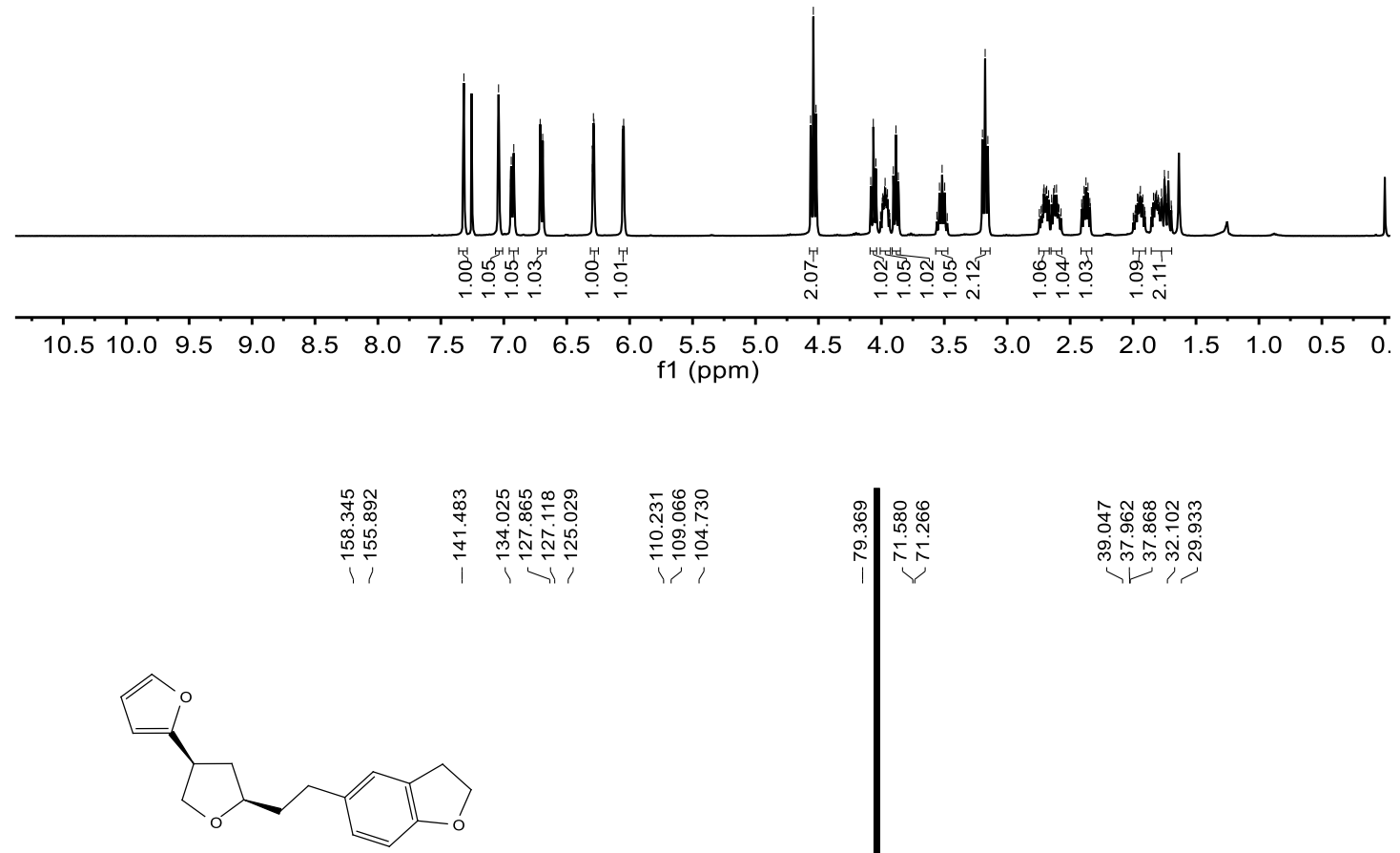

$(2 R, 4 S)-7$

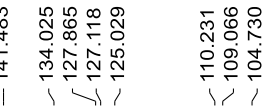

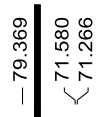

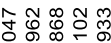

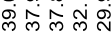

?

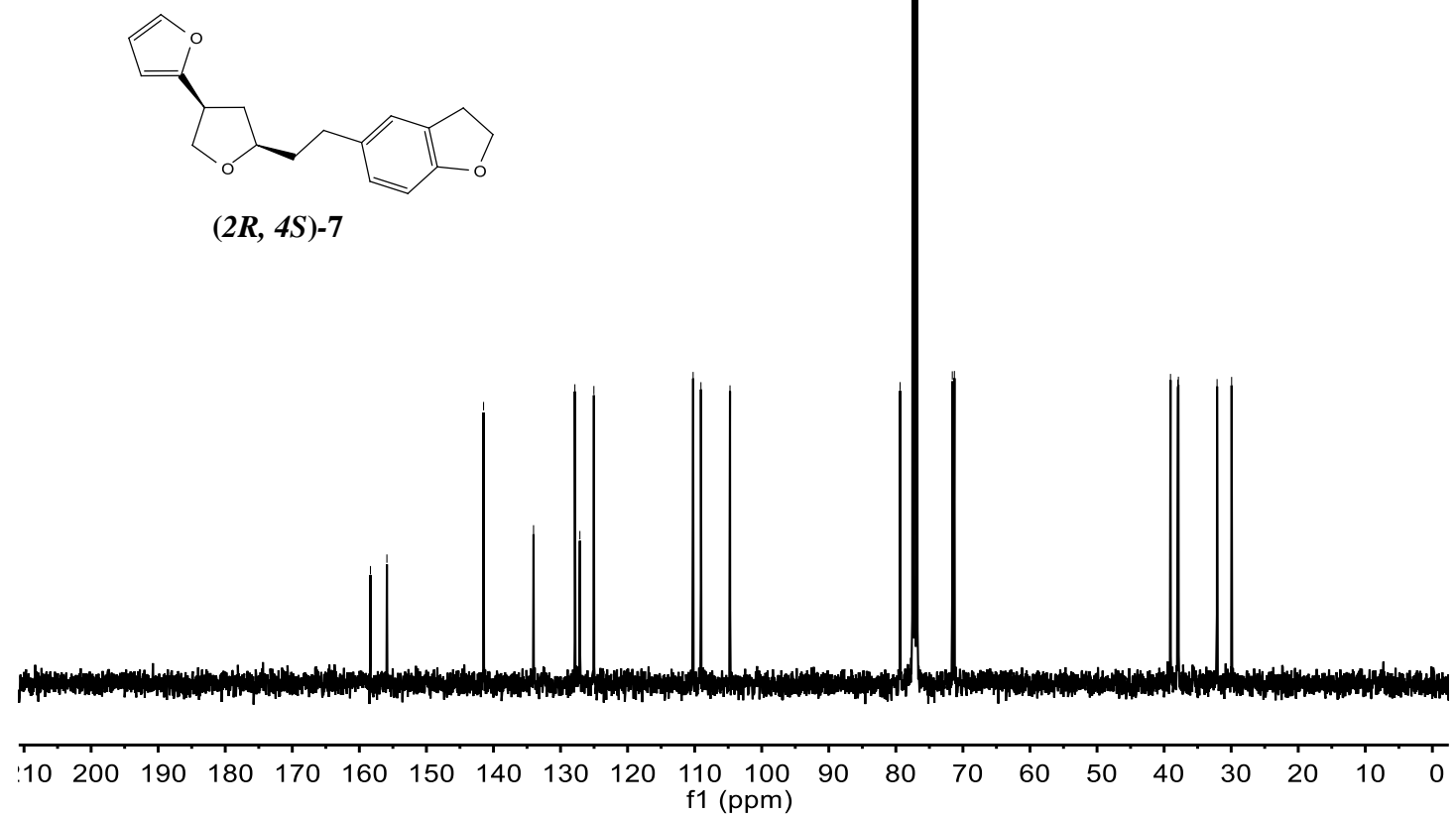




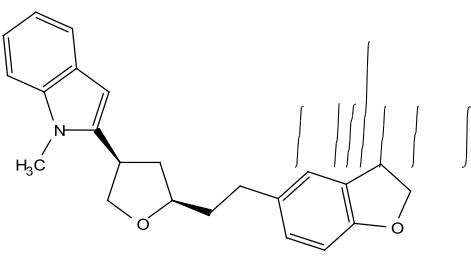

$(2 R, 4 S)-8$
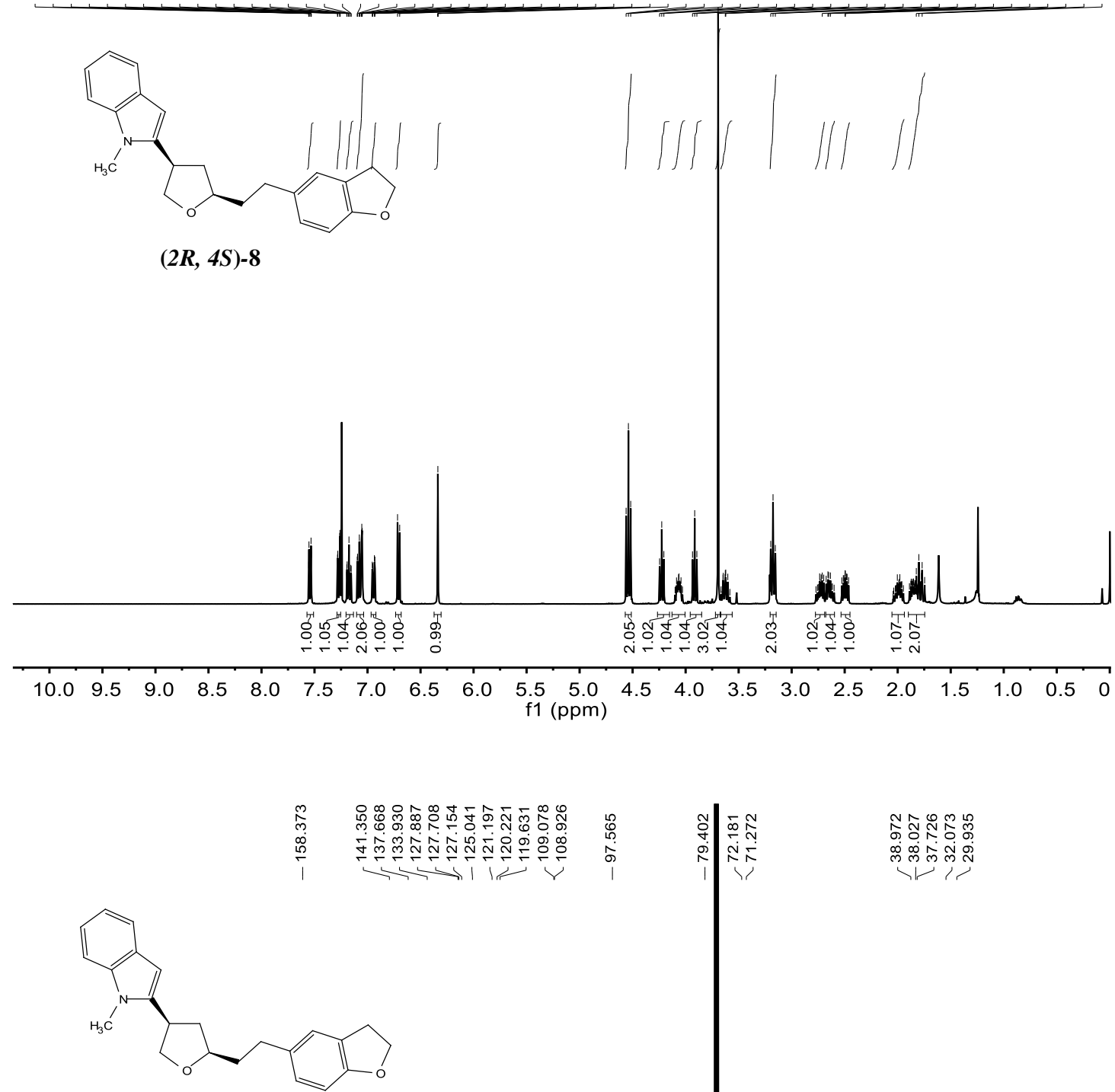

$(2 R, 4 S)-8$ 


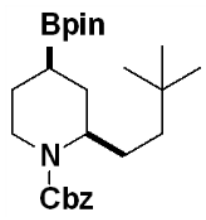

4bh
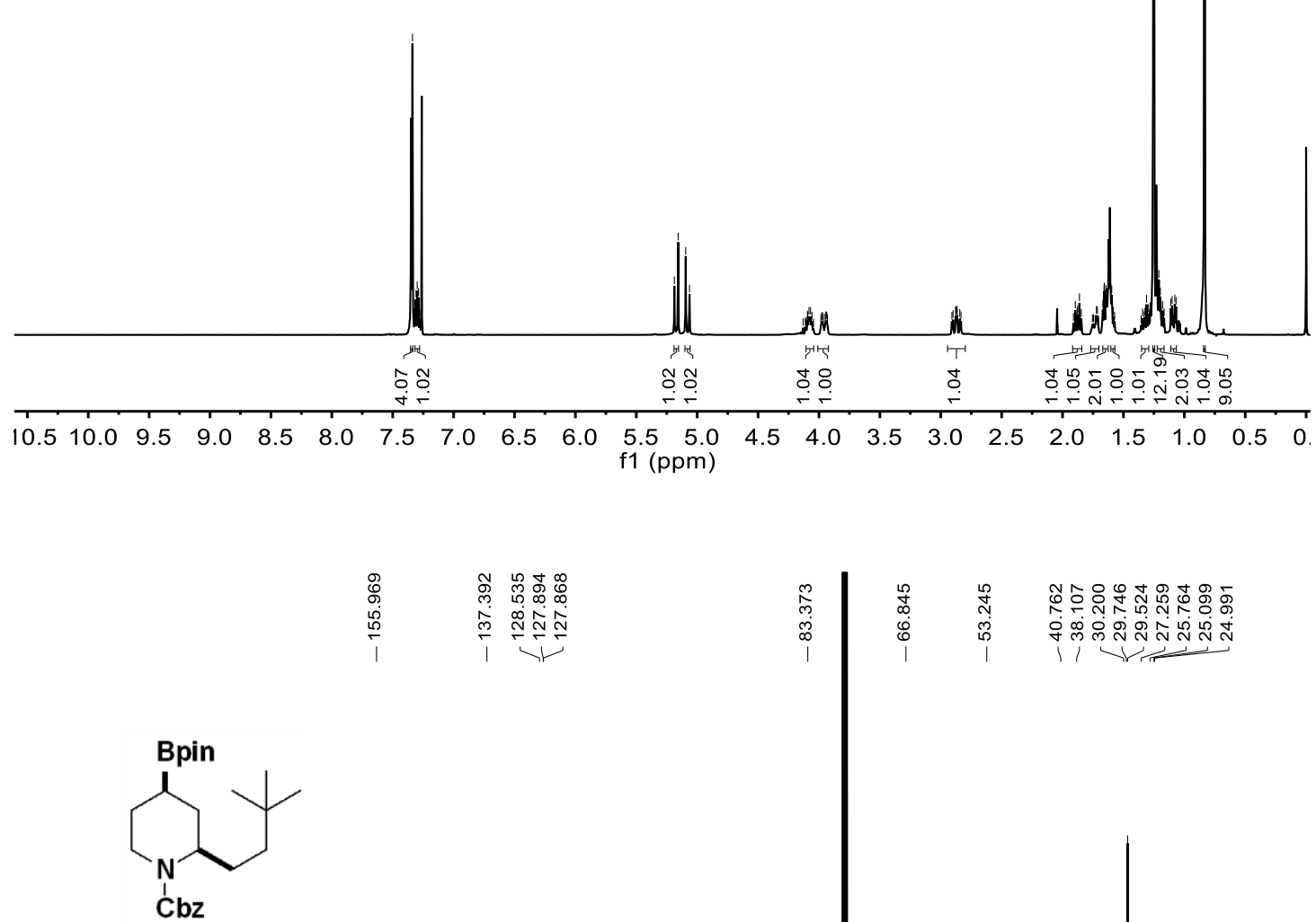

4bh

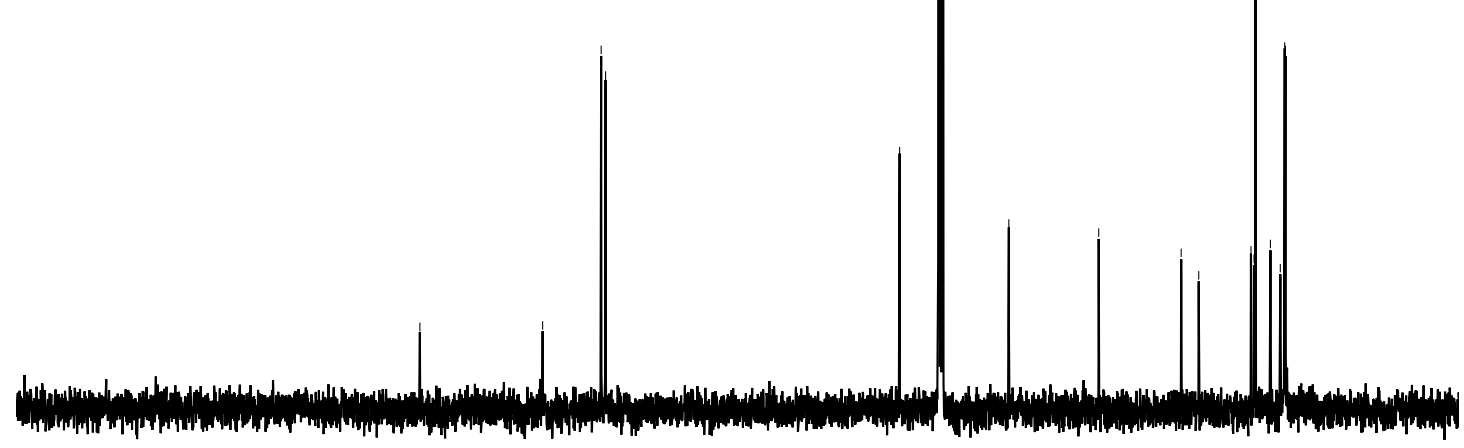

$\begin{array}{llllllllllllllllllllll}210 & 200 & 190 & 180 & 170 & 160 & 150 & 140 & 130 & 120 & 110 & 100 & 90 & 80 & 70 & 60 & 50 & 40 & 30 & 20 & 10 & \mathrm{C}\end{array}$ f1 (ppm) 


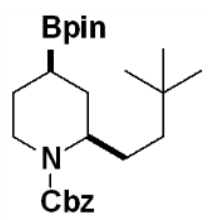

$4 b h$
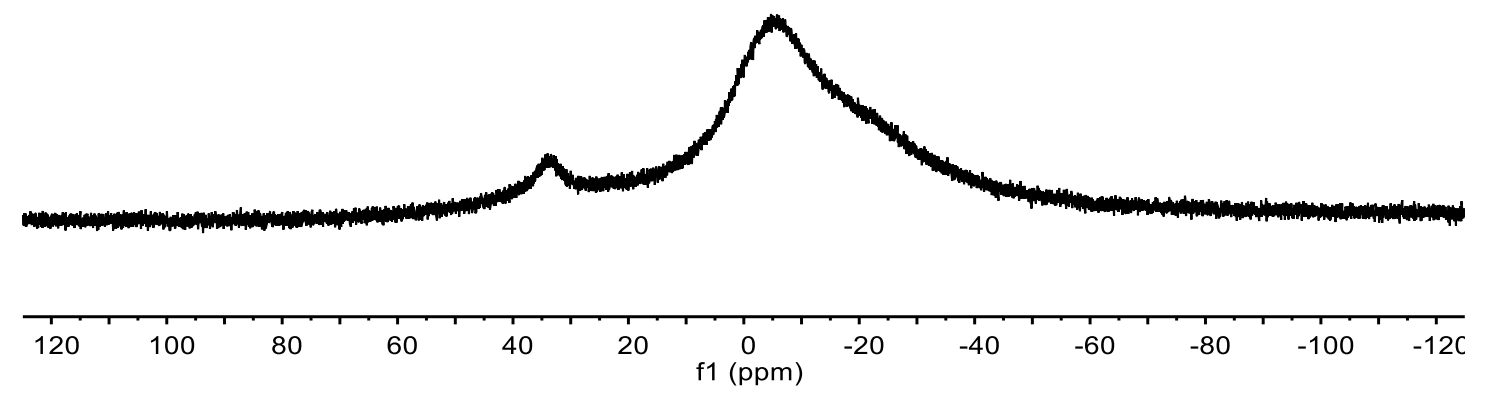

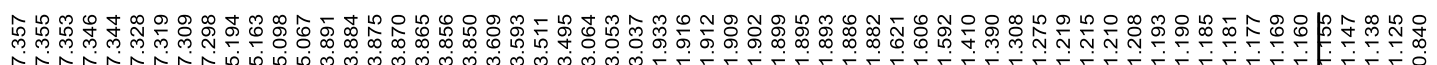

r raknknkw

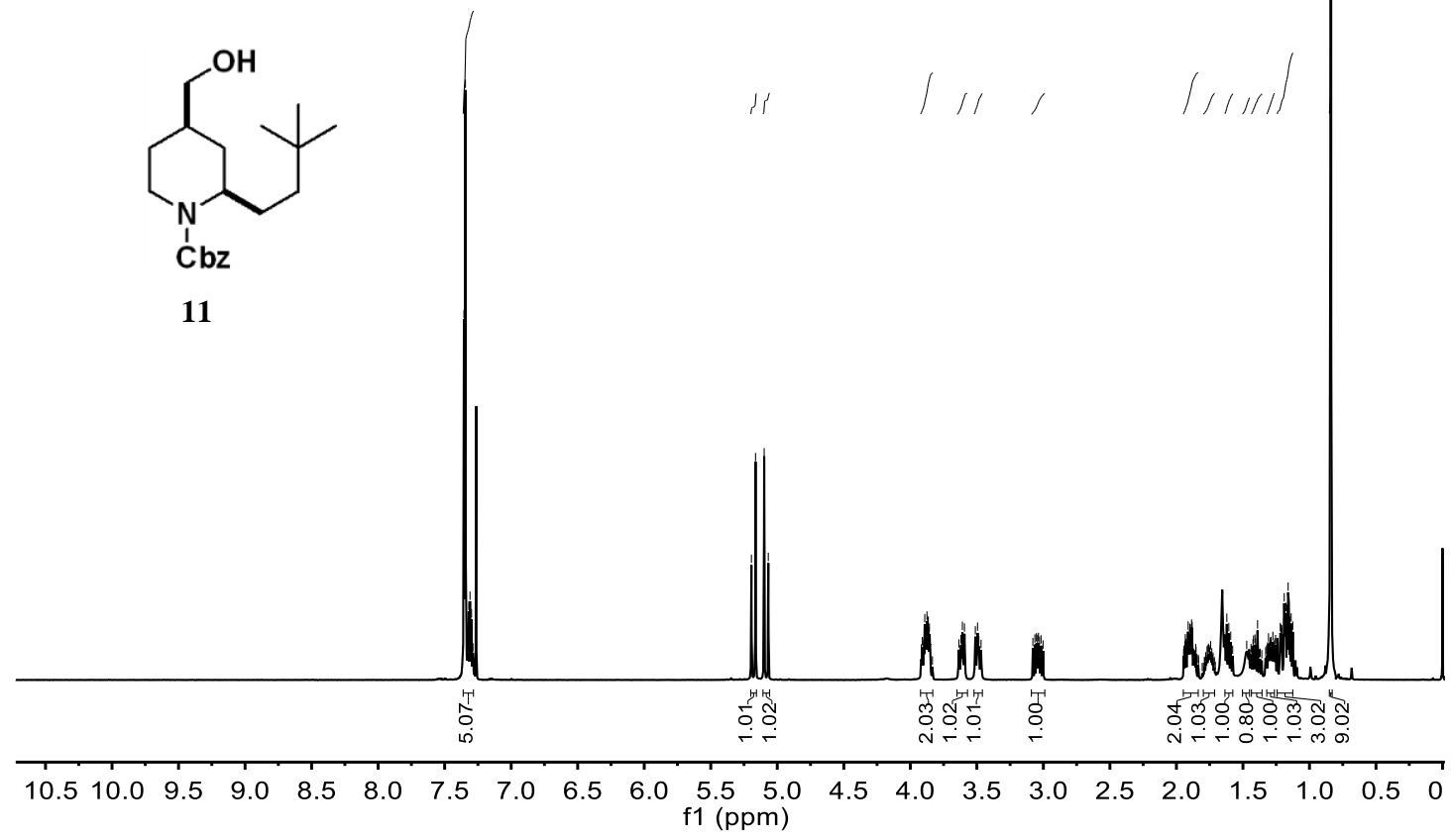



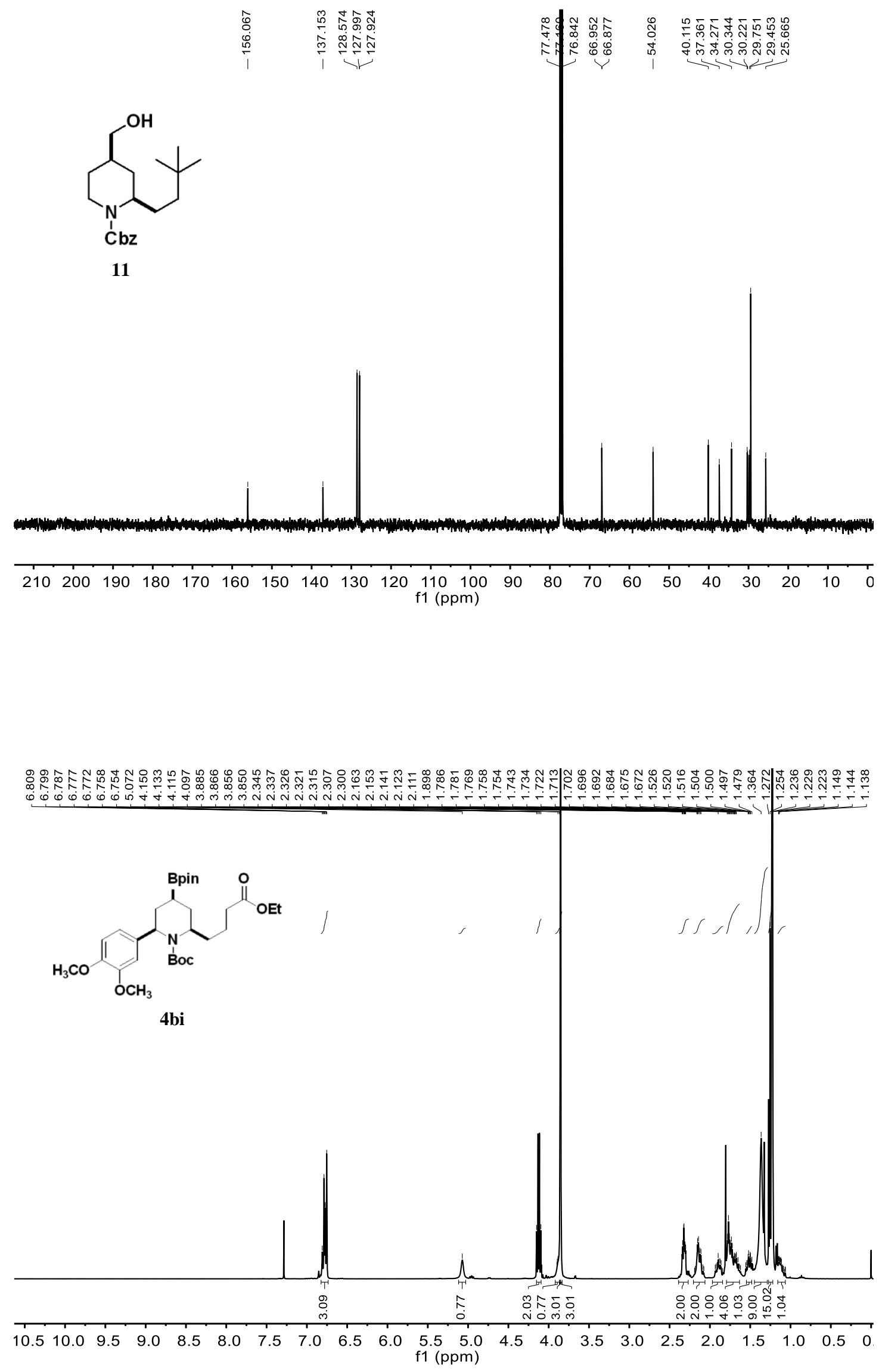


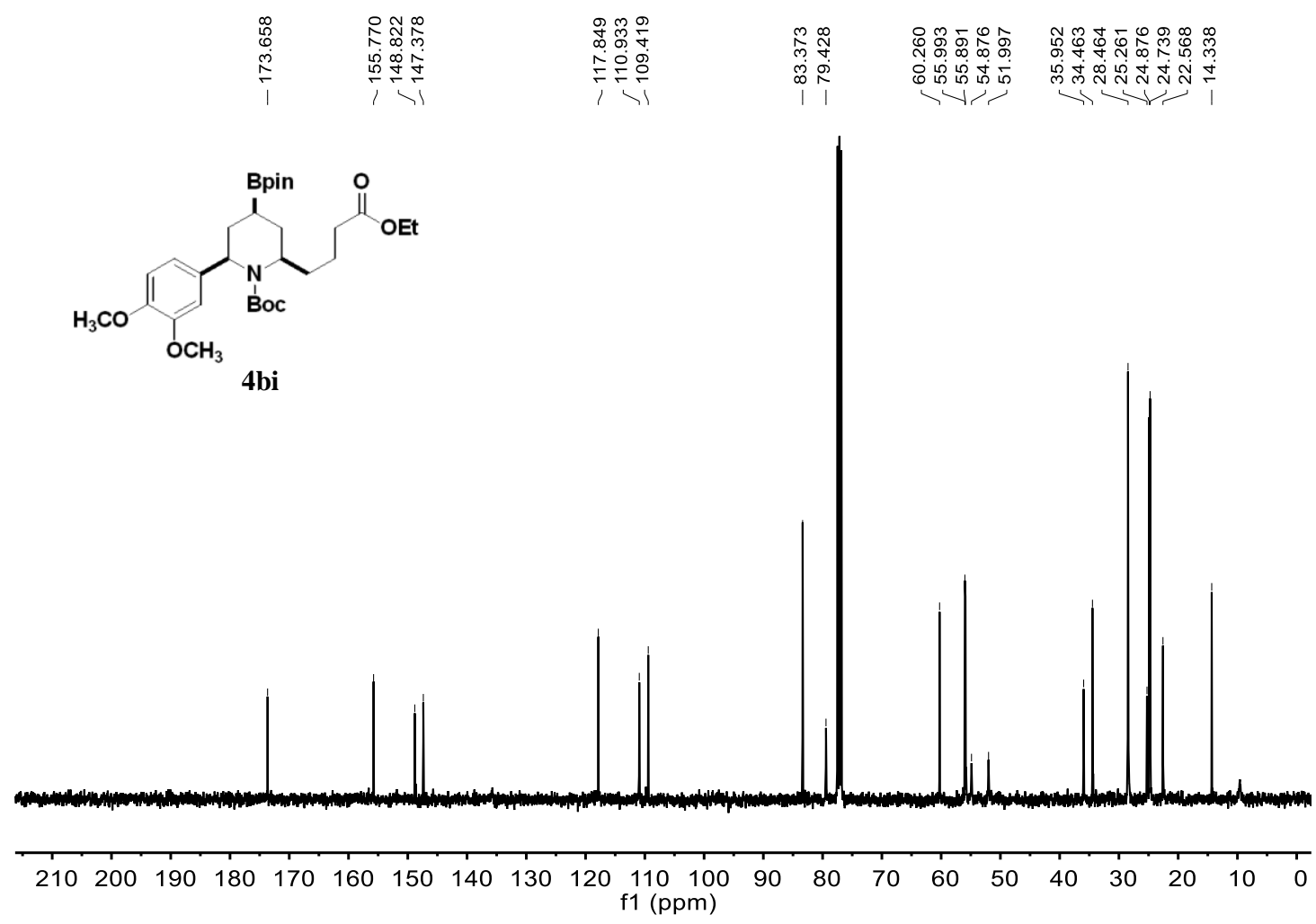

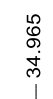<smiles>CCOC(=O)CCCC1CC(c2ccccc2)CC(c2ccc(OC)c(OC)c2)N1C(=O)OCc1ccccc1</smiles>

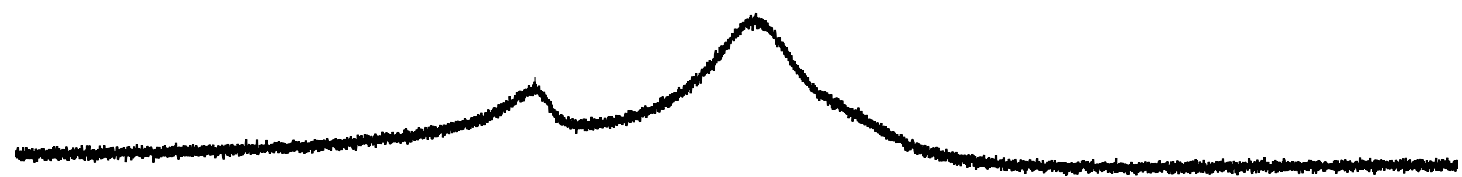

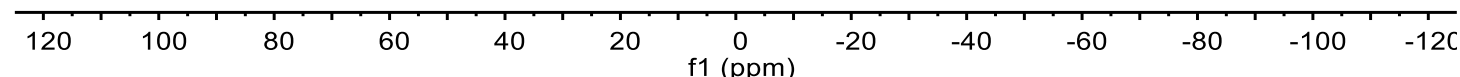




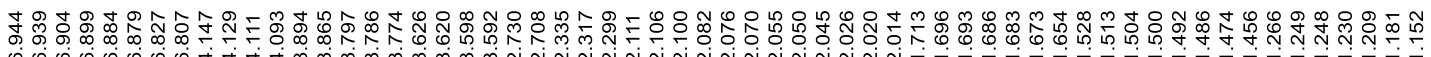
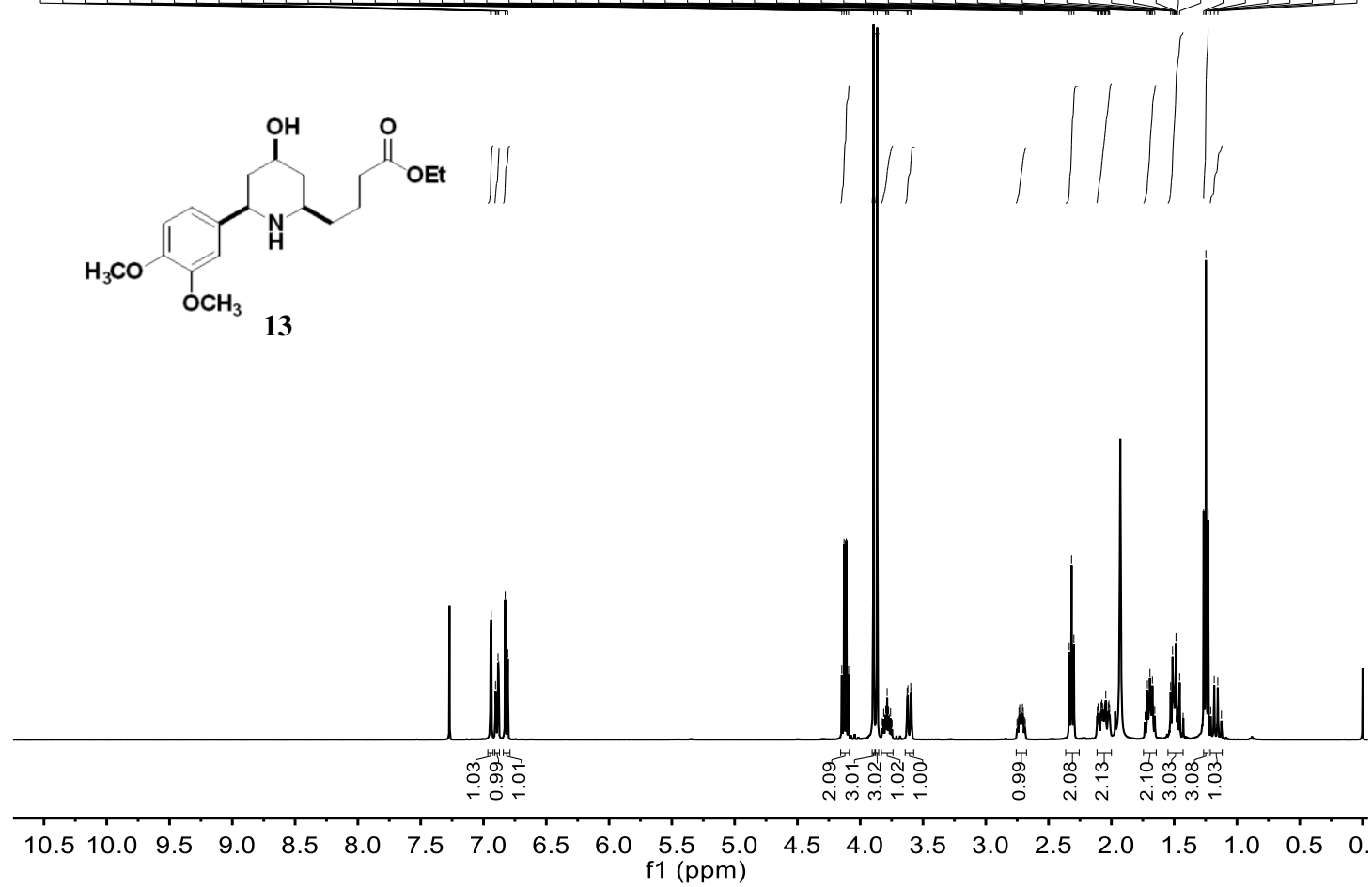<smiles>CCOC(=O)CCCC1CC(O)CC(c2ccc(OC)c(OC)c2)N1</smiles>

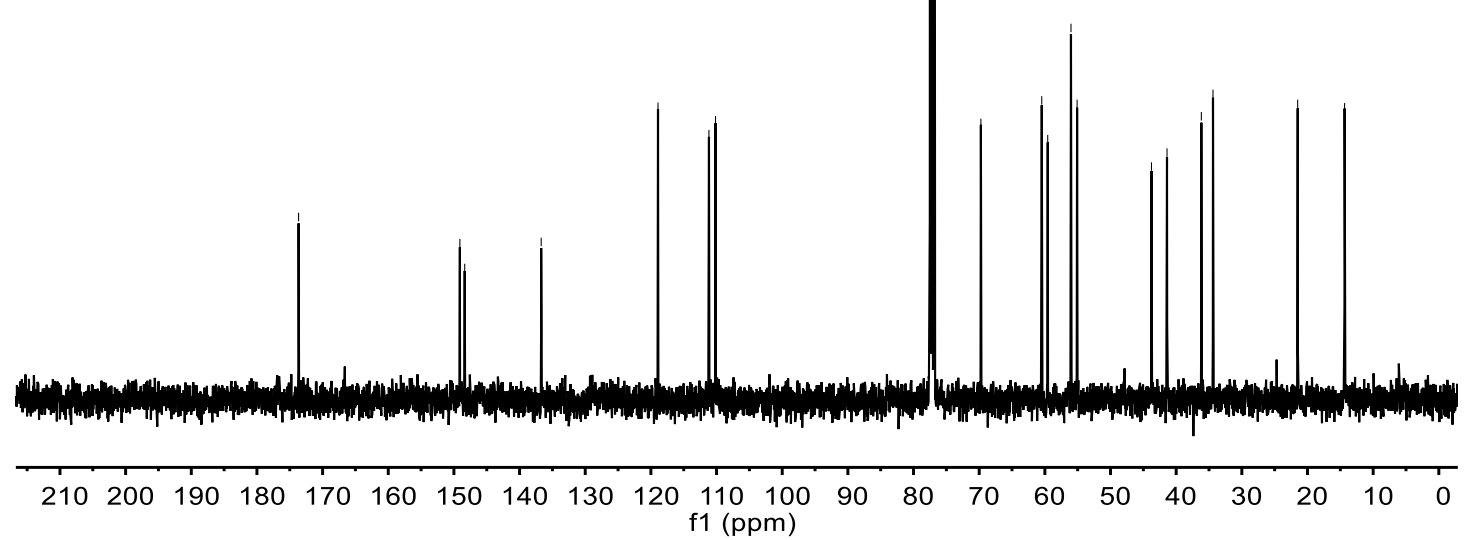




\section{Bpin}<smiles>CC1CCC(CCCc2ccccc2)N([O+])CC1</smiles>

18

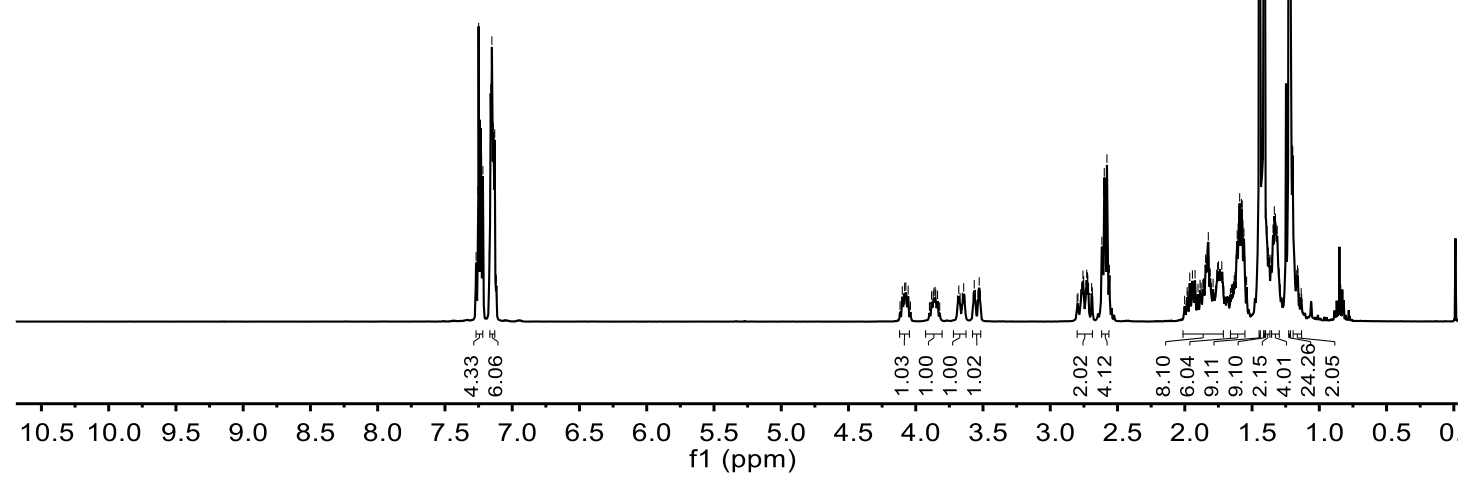

Bpin

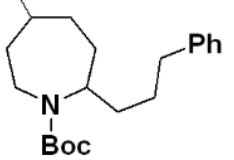

18

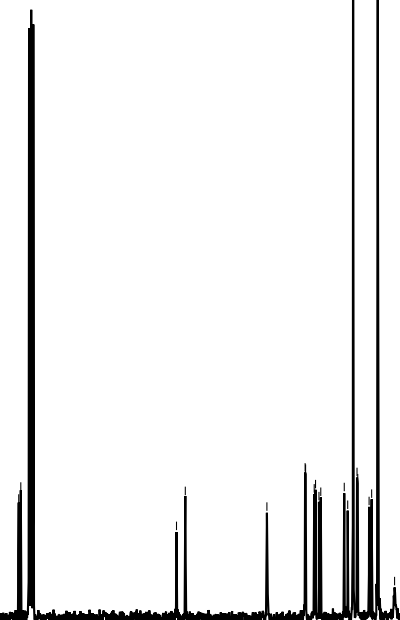

$\begin{array}{llllllllllllllllllllll}210 & 200 & 190 & 180 & 170 & 160 & 150 & 140 & 130 & 120 & 110 & 100 & 90 & 80 & 70 & 60 & 50 & 40 & 30 & 20 & 10 & 0\end{array}$ f1 (ppm) 


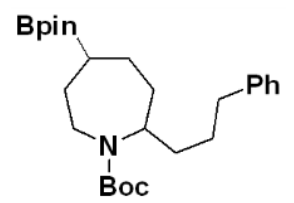

18
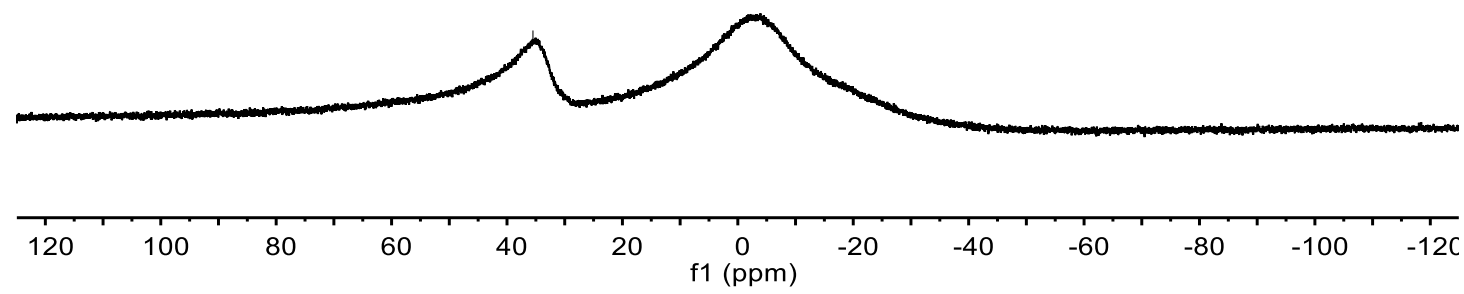

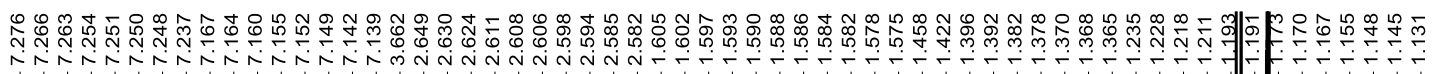

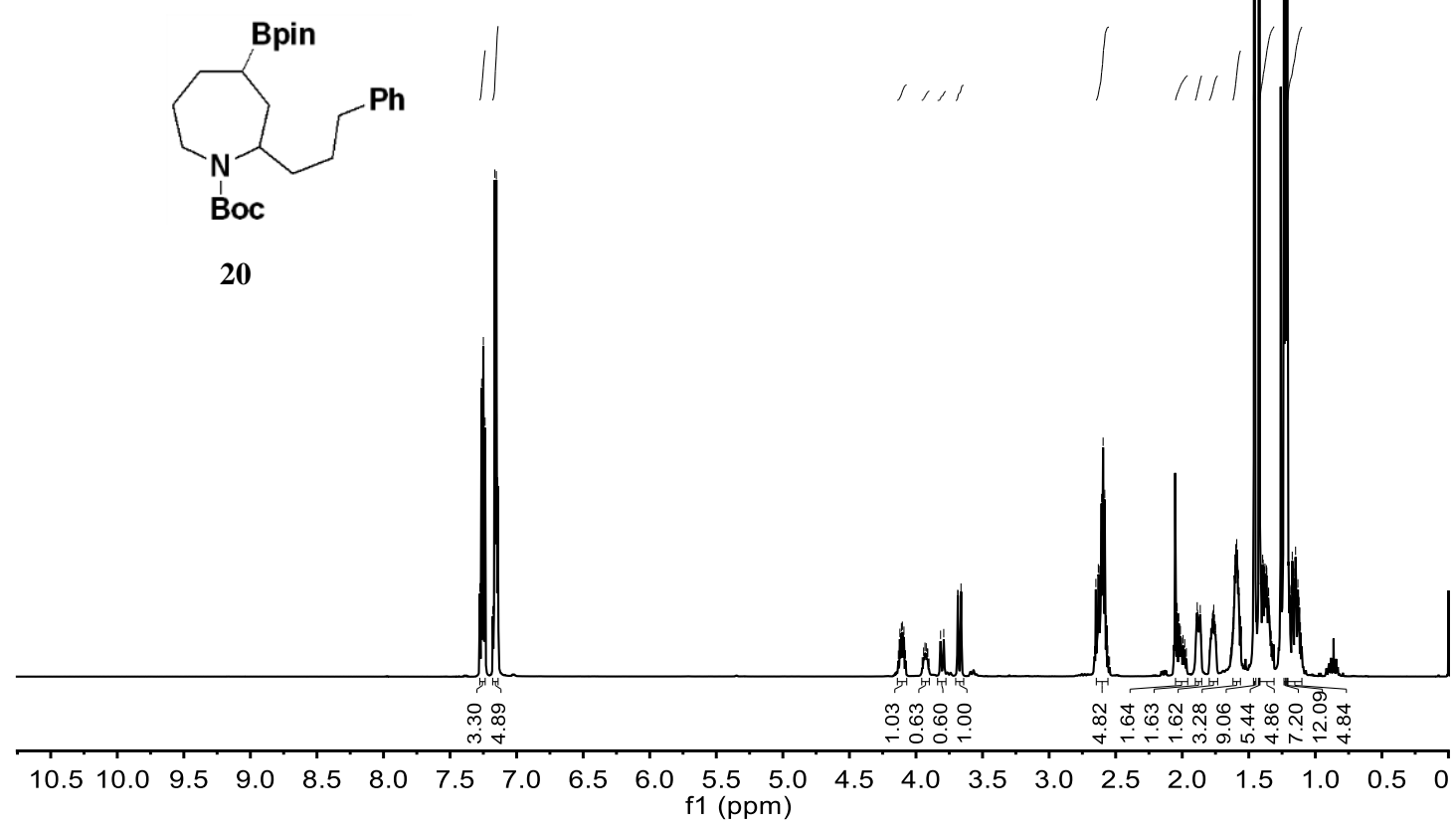




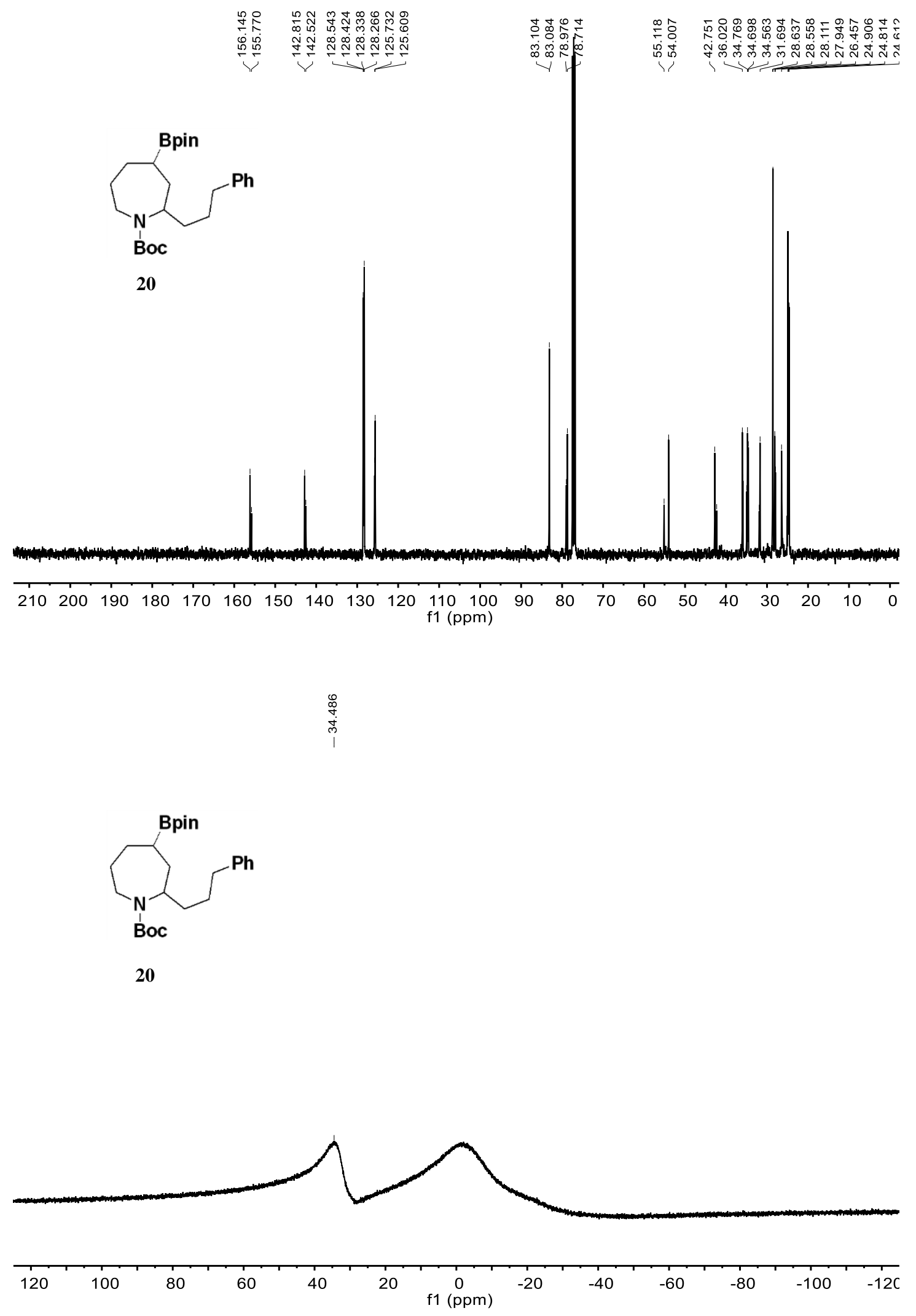




\section{References}

1. Xi, Y.; Hartwig, J. F. Diverse Asymmetric Hydrofunctionalization of Aliphatic Internal Alkenes through Catalytic Regioselective Hydroboration. J. Am. Chem. Soc. 2016, 138, 6703-6706.

2. Wu, J.; Guo, L.; Noble, Adam.; Aggarwal, V. K. Photoinduced Deoxygenative Borylat-ions of Aliphatic Alcohols. Angew. Chem. Int. Ed. 2019, 58, 18830-18834.

3. Yang, C.; Zhang, Z.; Liu, L. Copper-Catalyzed Cross-Coupling of Nonactivated Secondary Alkyl Halides and Tosylates with Secondary Alkyl Grignard Reagents. J. Am. Chem. Soc. 2012, 134, 11124-11127.

4. Sun, S.; Martin, R. Nickel-Catalyzed Umpolung Arylation of Ambiphilic $\alpha$-Bromoalkyl Boronic Esters. Angew. Chem. Int. Ed. 2018, 57, 3622-3625.

5. Pang, H.; Wu, D.; Cong, H.; Yin, G. Stereoselective Palladium-Catalyzed 1,3-Arylboration of Unconjugated Dienes for Expedient Synthesis of 1,3-Disubstituted Cyclohexanes. ACS Catal. 2019, 9, 8555-8560.

6. Bonet, A.; Odachowski, M.; Leonori, D.; Essafi, S.; Aggarwal, V. K. Enantiospecific sp ${ }^{2-}$ $\mathrm{sp}^{3}$ Coupling of Secondary and Tertiary Boronic Esters. Nat. Chem. 2014, 6, 584-589.

7. Mykura, R. C.; Aggarwal, V. K. Studies on the Lithiation, Borylation, and 1,2-Metalate Rearrangement of O-CycloalkSyl 2,4,6-Triisopropylbenzoates. Angew. Chem. Int. Ed. 2021, 60, 11436-11441.

8. Hoffmann, R. W.; Endesfelder, A. Stereoselective Intramolecular Nitrone Cycloaddition in the Synthesis of Lasubine II. Liebigs. Ann. Chem. 1986, 12, 1823-1836. 